\title{
Tracking of Animals Using Airborne Cameras
}

Clas Veibäck

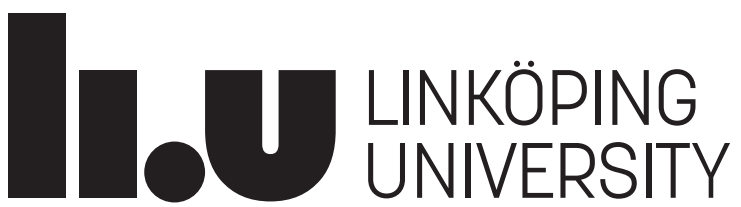


This is a Swedish Licentiate's Thesis.

Swedish postgraduate education leads to a Doctor's degree and/or a Licentiate's degree.

A Doctor's Degree comprises 240 ECTS credits (4 years of full-time studies).

A Licentiate's degree comprises 120 ECTS credits,

of which at least 60 ECTS credits constitute a Licentiate's thesis.

Linköping studies in science and technology. Thesis

No. 1761

Tracking of Animals Using Airborne Cameras

Clas Veibäck

\author{
clas.veibackliu.se \\ ww. control.isy.liu.se \\ Department of Electrical Engineering \\ Linköping University \\ SE-581 83 Linköping \\ Sweden
}

ISBN 978-91-7685-671-0

ISSN 0280-7971

Copyright (C) 2016 Clas Veibäck

Printed by LiU-Tryck, Linköping, Sweden 2016 
To family and friends 



\section{Abstract}

The various elements of a modern target tracking framework are covered. Background theory on pre-processing, modelling and estimation is presented as well as some novel ideas on the topic by the author. In addition, a few applications are posed as target tracking problems for which solutions are gradually constructed as relevant theory is covered.

Among considered problems are how to constrain targets to a region, use stateindependent measurements to improve estimation in jump Markov models and how to incorporate observations sampled at an uncertain time into a state-space model.

A framework is developed for tracking dolphins constrained to a basin using an overhead camera that suffers from occlusions. In this scenario, conventional motion models would suffer from infeasible predictions outside the basin. A motion model is developed for the dolphins where collisions with nearby walls are avoided by turning. The basin is modelled as a polygon where each point along the edge influences the turn rate of the dolphin. The proposed model results in predictions inside the basin, increasing robustness against occlusions. An extension to a Gaussian mixture background model providing a degree of confidence for detections is used to improve tracking in the presence of shadows. A probabilistic data association filter is also modified to estimate the dolphin extension as an ellipse. The proposed framework is able to maintain tracks through occlusions and poor light conditions.

A framework is developed for estimating takeoff times and directions of birds in circular cages using an overhead camera. A jump Markov model is used to model the stationary and flight behaviours of the birds. A proposed extension also incorporates state-independent measurements, such as blurriness, to improve mode estimation. Takeoff times and directions are estimated from mode transitions and results are compared to manually annotated data.

The cameras are inaccessible in both applications, disallowing proper calibrations. As an alternative, a method is proposed to estimate stationary camera models from available data and known features in the scene. A map of the basin and the funnel dimensions are used respectively. The method estimates a homography and distortion parameters in an invertible mapping function.

An extension to the linear Gaussian state-space models is proposed, incorporating an additional observation with an uncertain timestamp. The posterior distribution of the states is derived for the model, which is shown to be a mixture of Gaussians, as well as some estimators for the distribution. The effects of incorporating the observation with an uncertain timestamp into the model are analysed for a one-dimensional scenario. The model is also applied to improve the GPS position of an orienteering sprinter by using the control position as an observation with an uncertain timestamp. 



\section{Populärvetenskaplig sammanfattning}

Målföljning (eng. target tracking) är ett moget forskningsområde med anor tillbaka till åtminstone 30-talet. Då tävlade en handfull nationer på blodigt allvar om att snabbast kunna upptäcka fienden innan det var för sent. Traditionellt sett har målföljning fortsatt att vara starkt förknippat med militära tillämpningar och det är först på senare år som billiga och kommersiellt tillgängliga sensorer har öppnat upp för en mängd betydligt fredligare användningsområden.

Målföljning skulle kunna beskrivas som lokalisering av främmande objekt genom att samla in data från sensorer. Den här avhandlingen behandlar målföljning av olika sorters djur där data framförallt samlas in med videokameror. Det finns två bakomliggande syften vad gäller denna målföljning av djur. Det ena handlar om att underlätta forskning för biologer och det andra handlar om att skapa tekniska lösningar för att underlätta skyddet av sällsynta djur.

Biologer lägger ofta en ansenlig mängd tid på att samla in och sortera data. Det är tid som kan spenderas på mer givande forskningsaktiviteter. Med videokamera, bildbehandling och moderna algoritmer för målföljning är det möjligt att i viss mån automatisera datainsamlingen. Med automatisering kan mer information samlas in än med traditionella metoder och längre experiment kan ofta genomföras. Ytterligare en fördel är att man kan minska påverkan på djuren.

Parkvakterna i många nationalparker kämpar dagligen med intrång från tjuvjägare. De har ytterst begränsade resurser och utsätter sina liv för stor fara. Bestånden minskar fortfarande för många djurarter som går en mörk framtid till mötes. För att vända trenden behövs stora insatser på flera fronter samtidigt. Målföljning kan bidra med att på ett kostnadseffektivt sätt tillhandahålla övervakning av nationalparker. Kännedom om var djuren befinner sig underlättar koordinering av parkvakternas insatser för att skydda djuren. Målföljning kan ske med ett flertal olika sensorer, såsom radarer, fast uppsatta och luftburna videokameror, mikrofoner som lyssnar efter djurläten och även vittnesmål från parkvakterna. All insamlad information bidrar till att skapa en helhetsbild av situationen i nationalparken om den används rätt.

Att skapa en fullständig och användbar lösning för biologer och parkvakter är ett väldigt ambitiöst mål. I avhandlingen presenteras bakomliggande teori för målföljning varvat med författarens egna forskningsbidrag och lösningar för en handfull tillämpningar.

Den första tillämpningen som presenteras är ett samarbete med Kolmårdens djurpark. Biologer i djurparken studerar delfiners beteende i fångenskap. I dagsläget markerar studenter för hand i video var delfinerna befinner sig i bassängen. Med målföljning samlas djurens positioner in automatiskt utan mänsklig inblandning. Det främsta bidraget i forskningen är utvecklingen av en modell för hur delfinerna rör sig i bassängen.

Den andra tillämpningen som presenteras är ett samarbete med biologer vid Lunds universitet som studerar beteendet hos flyttfåglar. I en metod från 60-talet 
mäts fåglars rörelser i en tratt. Från repor i tratten som orsakats vid fåglarnas lyftförsök analyserar man riktningen för lyftförsöken. Med videokamera och målföljning samlas djurens positioner in och enskilda lyftförsök detekteras automatiskt. Det främsta bidraget i forskningen är en metod för att bättre utnyttja information från videon till att detektera lyftförsöken.

Den tredje tillämpningen som presenteras är ett första försök i Smarta savanner. Projektet utförs i samarbete med en nationalpark i Kenya för att lokalisera noshörningar från luften. Savannen på Kolmårdens djurpark har filmats med värmekamera från en obemannad flygande farkost för att samla in data under förhållanden som är snarlika de i nationalparken. Målföljning används sedan för att detektera och positionera savanndjuren i videon.

Den sista tillämpningen som presenteras kommer från en idé om att använda parkvakternas vittnesmål om kvarlämningar från noshörningar för att förbättra målföljningen. Å ena sidan är data från videokameror och radarer väldigt noggranna i tid, men relativt osäkra i de uppmätta positionerna. $\AA$ andra sidan kan positionen för en kvarlämning mätas noggrant samtidigt som det ofta är svårt att avgöra när noshörningen var på platsen. Genom att utnyttja informationen från båda källorna kan noshörningars förflyttningar i parken kartläggas bättre. Den bakomliggande teorin för observationer med osäker tid inom målföljning är relativt outforskad. Det främsta bidraget i forskningen är utvecklingen av en metod för att utnyttja sådana observationer. Ett enkelt teoretiskt fall används för att analysera metoden. Metoden utvärderas även i en tillämpning för att förbättra GPS-positioneringen av en orienterare genom att noggrant mäta positionen på en kontroll. 


\section{Acknowledgments}

I would like to express my gratitude to my supervisor Dr. Gustaf Hendeby for always having time for discussions, day and night, despite your high workload. I would also like to thank my co-supervisor Prof. Fredrik Gustafsson for your never-ending optimism and resourcefulness. The two of you together make an awesome radarpar of realism and optimism.

I would like to thank Prof. Svante Gunnarsson for hiring me as a PhD student and maintaining an amazing work environment, and Ninna for making things run smoothly. Working in the Automatic Control group is a pleasure.

I am very grateful for all the comments, input and proofreading provided by Dr. Gustaf Hendeby, Prof. Fredrik Gustafsson, Dr. Martin Skoglund, Dr. Zoran Sjanic and Mia Wallberg. Any remaining mistakes are my own. Also, thanks are in order for Dr. Gustaf Hendeby and Dr. Henrik Tidefelt for providing the $\mathrm{HAT}_{\mathrm{E}} \mathrm{X}$ class that has been used to write the thesis.

I am grateful for the financial support given by Vinnova Industry Excellence Center LINK-SIC.

The time at Automatic Control would be less amazing if it were not for all the awesome colleagues. Special gratitude goes to Zoran Sjanic, Hanna Nyqvist, Jonas Linder and Marek Syldatk for always being up for something when the work day is over and a decent distraction is needed. The absence of lower and upper limits on the level of discussions is both very entertaining and rewarding. I would also like to thank my room mate Martin Skoglund for lighting up the day, listening to rants, answering questions and introducing me to fine music. I am very grateful for the remaining colleagues, many of whom I have come to call my friends, Gustaf Hendeby, Christian Andersson Naesseth, Manon Kok, Johan Dahlin, Isak Nielsen, Sina Khoshfetrat-Pakazad, André Carvalho Bittencourt, Niclas Evestedt, Ylva Jung, Patrik Axelsson, Daniel Petersson, Karl Granström, Erik Hedberg, Martin Lindfors, Gustav Lindmark, Oskar Ljungqvist, Niklas Wahlström, Daniel Simon, Roger Larsson, Tohid Ardeshiri and Emre Özkan among others. Thank you for fun company on various trips, at the pub and in the fika room.

John, Matilda, Vincent and Charles, I am privileged to count you as my closest friends. When life came crashing down, you were there to help me pick up the pieces. My life would not be what it is without your support.

Mor, far and syster, I am very grateful for your support over the years and especially for the many amazing trips we have taken together.

Mia and Sam, all of a sudden you came into my life and lifted my spirit. I am so happy for your love and support and the life we are building together.

Thank you all for being the amazing persons that you are!

Linköping, October 2016 Clas Veibäck 



\section{Contents}

Notation

xiii

1 Introduction 1

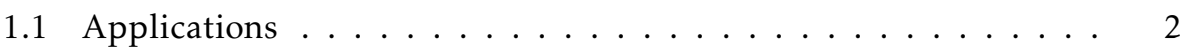

1.2 Target Tracking . . . . . . . . . . . . . . . . . 7

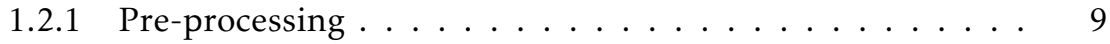

1.2.2 Association ...................... 9

1.2 .3 Estimation ................................ 10

1.2.4 Track Management . . . . . . . . . . . . . . 10

1.3 Motivation and Background ............... . . . . . 11

1.3.1 Dolphin Application ...................... 12

1.3.2 Bird Application . . . . . . . . . . . . . . . . 13

1.3.3 Savanna Application ...................... 14

1.3.4 Uncertain Time Scenarios . . . . . . . . . . . . . . . 14

1.4 Contributions and Publications . . . . . . . . . . . . . . 15

1.5 Thesis Outline . . . . . . . . . . . . . 17

2 State-Space Models 19

2.1 General Description . . . . . . . . . . . . . . . . . 20

2.2 Motion Models . . . . . . . . . . . . . . . . . . 21

2.3 Constrained Motion Model . . . . . . . . . . . . . . . . . . . . 24

2.3 .1 Motivation ................... 24

2.3.2 Region Model . . . . . . . . . . . . . . 25

2.3 .3 Motion Model . . . . . . . . . . . . . . . . 27

2.4 Sensor Models . . . . . . . . . . . . . . . . . . . . . . . . . . . . . . . . . . . 29

2.5 Jump Markov Model . . . . . . . . . . . . . . . . . . . . . . . . 32

2.5.1 General Model . . . . . . . . . . . . . . . . 32

2.5.2 Model Extension . . . . . . . . . . . . . . . 34

2.6 Extended Target Model . . . . . . . . . . . . . . . . . . 34

2.7 Uncertain Timestamp Model . . . . . . . . . . . . . . . 36

3 Camera Sensor $\quad 39$

3.1 Camera Model ....................... 39 
3.2 Stationary Camera Modelling _. . . . . . . . . . . . . . 41

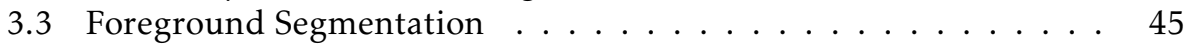

3.4 Data Reduction . . . . . . . . . . . . . . . . . . . 51

4 State-Space Estimation $\quad 55$

4.1 Bayesian Solution . . . . . . . . . . . . . . . . . 55

4.2 Kalman Filter . . . . . . . . . . . . . . . . . 56

4.3 Linear Gaussian Smoother … . . . . . . . . . . . . . . . . 59

4.3 .1 General Smoothing . . . . . . . . . . . . . . . . . 59

4.3.2 Update of Posterior Distribution . . . . . . . . . . . 61

4.4 Filter Bank . . . . . . . . . . . . . . . . . . . . . . . . 64

4.4.1 Filter Bank for the General Model . . . . . . . . . . . . . 65

4.4.2 Filter Bank for the Model Extension . . . . . . . . . . . 67

4.5 Extended Target Model Estimation . . . . . . . . . . . . . 71

4.6 Uncertain Timestamp Model Estimation . . . . . . . . . . . . . 75

4.6.1 Posterior Distributions . . . . . . . . . . . . . . . . 75

4.6 .2 Point Estimators . . . . . . . . . . . . . . . . 77

5 Data Association $\quad 89$

5.1 Data Association Uncertainty . . . . . . . . . . . . . . 90

5.1 .1 Uncertainty Models . . . . . . . . . . . . . . . . . . 90

5.1 .2 Track Score . . . . . . . . . . . . . . . . . . . . . . . . . 99

5.1 .3 Track Quality . . . . . . . . . . . . . . . . . 92

5.1 .4 Assignment Problem . . . . . . . . . . . . . . . . . 93

5.2 Multiple Hypothesis Tracker . . . . . . . . . . . . . . . . . . . . 95

5.3 Probabilistic Data Association Filter . . . . . . . . . . . . 98

5.3.1 Standard Probabilistic Data Association Filter . . . . . . . . 98

5.3 .2 Track Management . . . . . . . . . . . . . . . . . . . 99

5.3.3 Modified Probabilistic Data Association Filter . . . . . . . 100

6 Conclusions and Future Work 107

$\begin{array}{ll}\text { Bibliography } & 111\end{array}$ 
Notation

Mathematical Style

\begin{tabular}{cl}
\hline Notation & Meaning \\
\hline$a$ & Scalar variable or parameter \\
$\mathbf{a}$ & Vector variable or parameter \\
$\mathbf{A}$ & Matrix variable or parameter \\
$\mathcal{A}$ & Set variable or parameter \\
\hline
\end{tabular}




\section{Set Notation}

\begin{tabular}{cl}
\hline Notation & Meaning \\
\hline $\mathbb{N}$ & Set of natural numbers \\
$\mathbb{R}$ & Set of real numbers \\
$\mathbb{R}^{a}$ & Set of real column vectors of dimension $a$ \\
$\mathbb{R}^{a \times b}$ & Set of real matrices of dimension $a \times b$ \\
$\mathbb{R}^{+}$ & Set of positive real numbers \\
$S O(3)$ & Set of $3 \times 3$ orthogonal matrices with unit determinant \\
$S_{+}$ & Set of all positive semi-definite matrices \\
$S_{+}^{a}$ & Set of all positive semi-definite matrices of dimensions \\
& $a \times a$ \\
$S_{++}$ & Set of all positive definite matrices \\
$S_{++}^{a}$ & Set of all positive definite matrices of dimensions $a \times a$ \\
{$[a, b]$} & Set of all real numbers in an interval, $[a, b]=\{c \in$ \\
& $\mathbb{R} \mid a \leq c \leq b\}$ \\
$\{\mathbf{a} \mid \operatorname{condition}\}$ & Set of all vectors a such that the condition holds \\
$a: b$ or $\{a: b\}$ & Set of all natural numbers in an interval, $\{a: b\}=\{c \in$ \\
& $\mathbb{N} \mid a \leq c \leq b\}$ \\
$\left\{\mathbf{a}_{b}\right\}_{b \in \mathcal{A}}$ & Set of all vectors $\mathbf{a}_{b}$ such that $b \in \mathcal{A}$ \\
$\left\{\mathbf{a}_{b}\right\}_{b=c}^{d}$ & Set of all vectors $\mathbf{a}_{b}$ such that $b \in\{c: d\}$ \\
$|\mathcal{A}|$ & Cardinality of set $\mathcal{A}$ \\
$\mathcal{A} \cup \mathcal{B}$ & Union of sets $\mathcal{A}$ and $\mathcal{B}$ \\
$\mathcal{A} \cap \mathcal{B}$ & Intersection of sets $\mathcal{A}$ and $\mathcal{B}$ \\
$\mathcal{A} \backslash \mathcal{B}$ & Difference of sets $\mathcal{A}$ and $\mathcal{B}$ \\
\hline
\end{tabular}

\section{Probability Notation}

\begin{tabular}{cl}
\hline Notation & Meaning \\
\hline$p_{\mathbf{a}}(\mathbf{a})$ or $p(\mathbf{a})$ & PDF or PMF of stochastic variable or expression a \\
$p(\mathbf{a} \mid \mathbf{b})$ & Conditional PDF or PMF of stochastic variable or ex- \\
& pression a given $\mathbf{b}$ \\
$\mathbf{a} \sim p(\mathbf{a})$ & Stochastic variable a follows distribution $p(\mathbf{a})$ \\
$\mathbf{E}(\mathbf{a})$ & Expected value of stochastic expression a \\
$\operatorname{Cov}(\mathbf{a})$ & Covariance of stochastic expression a \\
$\mathcal{N}(\mu, \Sigma)$ & Multivariate normal distribution with mean $\mu$ and co- \\
$\mathcal{N}(\mathbf{a} \mid \mu, \Sigma)$ & variance $\Sigma$ \\
$\mathcal{I} \mathcal{W}(\nu, \mathbf{V})$ & Inverse Wishart distribution with $v \in \mathbb{R}^{+}$degrees of \\
$\mathcal{I} \mathcal{W}(\mathbf{A} \mid \mathcal{v}, \mathbf{V})$ & freedom and matrix V $\in S_{++}$ \\
$\mathcal{P} \mathcal{S}(\lambda)$ & Poisson distribution with intensity $\lambda$ \\
$\mathcal{P S}(a \mid \lambda)$ & Poisson PMF of scalar $a$ \\
$\mathcal{U}(\mathcal{V})$ & Uniform distribution over the set $\mathcal{V}$ \\
$\mathcal{U}(\mathbf{a} \mid \mathcal{V})$ & Uniform PDF of vector $\mathbf{a}$ \\
\hline
\end{tabular}




\section{Vector and Matrix Notation}

\begin{tabular}{cl}
\hline Notation & Meaning \\
\hline $\mathbf{I}_{a}$ & Identity matrix of dimension $a \times a$ \\
$\mathbf{I}$ & Identity matrix of applicable dimension \\
$\mathbf{0}$ & Column vector of zeroes of applicable dimension \\
$\mathbf{A} \otimes \mathbf{B}$ & Kronecker product of matrices $\mathbf{A}$ and $\mathbf{B}$ \\
$\mathbf{a}^{T}$ or $\mathbf{A}^{T}$ & Transpose of vector a or matrix $\mathbf{A}$ \\
$\mathbf{a}_{\perp}$ & $\pi / 2$ rad clockwise rotation of two-dimensional vector \\
& a, such that $\mathbf{a}_{\perp}=\left(\begin{array}{c}a \\
b\end{array}\right) \perp=\left(\begin{array}{c}b \\
-a\end{array}\right)$ \\
$|\mathbf{A}|$ & Determinant of matrix $\mathbf{A}$ \\
$\operatorname{tr}(\mathbf{A})$ & Trace of matrix $\mathbf{A}$ \\
$\mathbf{a} \cdot \mathbf{b}$ & Scalar product, $\mathbf{a}^{T} \mathbf{b}$, of column vectors a and $\mathbf{b}$ \\
$\|\mathbf{a}\|$ & Norm, $\sqrt{\mathbf{a}^{T} \mathbf{a}, \text { of column vector } \mathbf{a}}$ \\
$\|\mathbf{a}\|_{\mathbf{A}}$ & Mahalanobis distance of vector $\mathbf{a}$, where $\mathbf{A}$ is positive \\
& definite, $\sqrt{\mathbf{a}^{T} \mathbf{A}^{-1} \mathbf{a}}$ \\
$\mathbf{\mathbf { a }}$ & Transforms a vector a to homogeneous coordinates, \\
& $\left(\mathbf{a}^{T}, 1\right)^{T}$ \\
$\mathbf{A}_{a, b}$ or $\mathbf{A}_{a b}$ & Element at row $a$ and column $b$ in matrix $\mathbf{A}$ \\
$a_{x}$ and $a_{y}$ & First and second element of vector a, respectively \\
$\operatorname{diag}\left(a_{1}, \ldots, a_{b}\right)$ & $\begin{array}{l}\text { Diagonal matrix with elements } a_{1}, \ldots, a_{b} \text { on the diag- } \\
\text { onal }\end{array}$ \\
\hline
\end{tabular}

\section{Other Notation}

\begin{tabular}{|c|c|}
\hline Notation & Meaning \\
\hline $\arctan _{2}(y, x)$ & $\begin{array}{l}\text { Arctangent with two arguments, returning output in } \\
\text { the correct quadrant }\end{array}$ \\
\hline$a \propto b$ & $\begin{array}{l}\text { Proportionality of expression } a \text { to expression } b \text {, such } \\
\text { that } a=c b \text { where } c \in \mathbb{R}^{+} \text {is a constant }\end{array}$ \\
\hline$\dot{\mathbf{a}}$ or $\dot{\mathbf{a}}(t)$ & Derivative of $\mathbf{a}(t), \frac{d \mathbf{a}}{d t}(t)$, with regards to time $t$ \\
\hline $\mathbf{a}^{+}$or $\mathbf{A}^{+}$ & $\begin{array}{l}\text { Denotes an update of a vector a or matrix } \mathbf{A} \text {, when } \\
\text { notation does not explicitly consider time }\end{array}$ \\
\hline$\lceil a\rceil$ & Ceiling of $a$ \\
\hline$a !$ & Factorial of $a$ \\
\hline
\end{tabular}




\begin{tabular}{|c|c|}
\hline Abbreviation & Meaning \\
\hline AWGNSSM & Additive White Gaussian Noise State-Space Model \\
\hline $\mathrm{CMM}$ & Constrained Motion Model \\
\hline СТМ & Coordinated Turn Model \\
\hline $\mathrm{EKF}$ & Extended Kalman Filter \\
\hline $\mathrm{EM}$ & Expectation-Maximization \\
\hline ETT & Extended Target Tracking \\
\hline GNN & Global Nearest Neighbour \\
\hline GNSS & Global Navigation Satellite System \\
\hline IEKF & Iterated Extended Kalman Filter \\
\hline IMM & Interacting Multiple Models \\
\hline IMU & Inertial Measurement Unit \\
\hline JMAP & Joint Maximum A Posteriori \\
\hline JMM & Jump Markov Model \\
\hline JPDA & Joint Probabilistic Data Association \\
\hline $\mathrm{KF}$ & Kalman Filter \\
\hline LGSSM & Linear Gaussian State-Space Model \\
\hline MAP & Maximum A Posteriori \\
\hline MHT & $\begin{array}{l}\text { Multiple Hypothesis Tracker or Multiple Hypothesis } \\
\text { Tracking }\end{array}$ \\
\hline MMSE & Minimum Mean Squared Error \\
\hline MSE & Mean Squared Error \\
\hline MTT & Multiple Target Tracking \\
\hline NCPM & Nearly Constant Position Model \\
\hline NCVM & Nearly Constant Velocity Model \\
\hline NSSM & Nonlinear State-Space Model \\
\hline NN & Nearest Neighbour \\
\hline OOS & Out Of Sequence \\
\hline PDA & Probabilistic Data Association \\
\hline PDF & Probability Density Function \\
\hline PF & Particle Filter \\
\hline PMF & Probability Mass Function \\
\hline PHD & Probability Hypothesis Density \\
\hline PSSM & Probabilistic State-Space Model \\
\hline RTSS & Rauch-Tung-Striebel Smoother \\
\hline SSM & State-Space Model \\
\hline SPRT & Sequential Probability Ratio Test \\
\hline STT & Single Target Tracking \\
\hline TBD & Track Before Detect \\
\hline UTM & Uncertain Timestamp Model \\
\hline UKF & Unscented Kalman Filter \\
\hline
\end{tabular}




\section{1}

Introduction

Target tracking is a mature area of research, which traditionally has had a strong focus on military applications. With the advent of cheap and commercially available sensors and computational power, new possibilities have opened up for target tracking to be useful in a broader group of applications.

The title of the thesis is motivated by the application of target tracking to different scenarios involving animals. Three of the scenarios involve animals recorded by overhead cameras. Tracking animal movement is an emerging application area of target tracking. The research of animal behaviour is in need of automatic tracking, where today tedious manual work is performed. Cross-disciplinary research between the target tracking community and biologists has the potential to automatically generate large amounts of animal data to the biologists, and at the same time it poses challenging problems for the target tracking community. Conservation of endangered species is another field in which target tracking of animals is needed. Target tracking can cost-effectively provide park rangers in wildlife reserves with situational awareness, improving the coordination of conservation efforts.

Specific animal tracking applications are considered in this thesis. Models and methods are tailored to the applications to allow for inference of position or behaviour of the animals. The applications are introduced in Section 1.1 and further motivation and background is presented in Section 1.3. The concept of target tracking is introduced in Section 1.2 as a framework for solutions to the applications. Contributions and publications by the author are discussed in Section 1.4 and the outline of the thesis is presented in Section 1.5. 


\subsection{Applications}

Various theoretical topics are covered in this thesis and five different applications are used to motivate and exemplify the theory. Solutions for the applications are developed as relevant theory is covered. The applications shall be seen as such and not as mere illustrations of concepts. The structure of the thesis is perhaps unconventional in the sense that results are not presented in an individual chapter, but are presented for each application in the last chapters when all necessary theory has been covered.

The first three applications are animal tracking problems involving cameras. The fourth application is technically not an application, but a simple scenario used for analysis and will at times be referred to as an application for simplicity. The underlying problem in the last applications stems from an animal tracking problem, but since no relevant data from the considered animal tracking application has been collected, data from an orienteering sprinter is used instead.

Application 1.1 introduces a scenario where biologists want to track dolphins in captivity to observe their behaviour. The main contribution presented with this application is a motion model tailored to targets in captivity. The proposed solution for this application and related background theory were previously published by the author in [112, (C) 2015 IEEE] .

\section{Application 1.1: Tracking of Dolphins}

Dolphins swimming in a basing are tracked in this challenging application using a fisheye camera mounted in the ceiling. See Figure 1.1 for an illustration of available footage. The biological motivation [111] is to understand how the behavioural pattern is affected by underwater sonar transponders. A better understanding can be obtained with this research of how the dolphin's internal navigation system works. Today, tracking is performed by manually annotating the positions of the dolphins in the video.

The application is a classic target tracking problem, with false detections from pre-processing, individuals forming tight groups and a need to model the extension of targets. New challenges include shadows at the bottom of the basin and sun light through the ceiling windows that causes large local changes in light conditions. The scene includes hard constraints caused by the basin, occlusion from a platform in the water and missed detections caused by a limited field of view from the fisheye camera. Another challenge is the difficulty to obtain sufficient data to calibrate the camera.

The data is generously provided by Kolmården Wildlife Park. All application descriptions in the thesis with the word dolphin in the caption are related to this application. Results are presented throughout the thesis and the final results of the solution are presented in Application 5.3.

Application 1.2 introduces a scenario where biologists want to track the movements of a bird in an Emlen funnel [45] to observe their behaviour. The main 


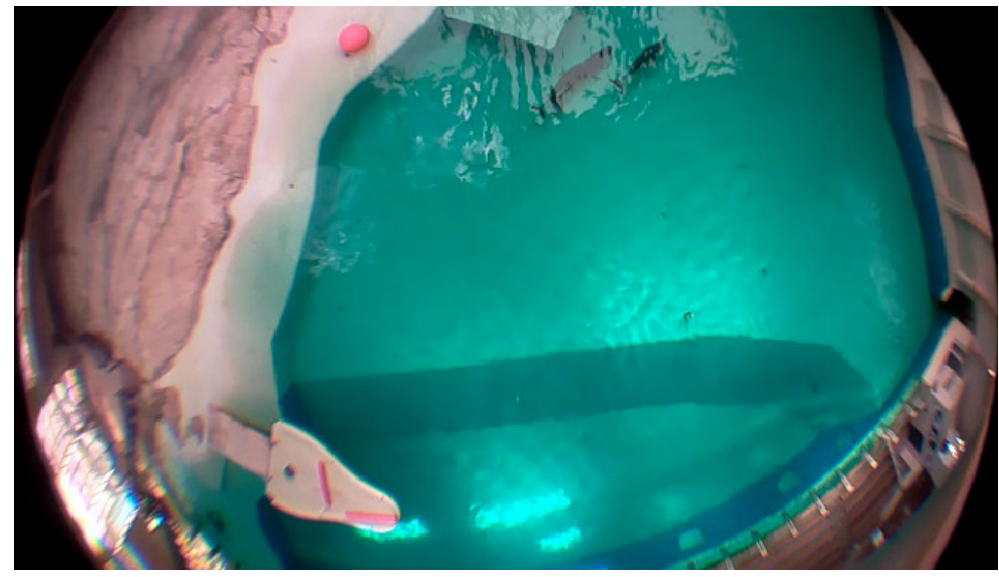

Figure 1.1: A single frame from a video recorded of the dolphins by the fisheye camera. Some of the challenges inherent to this problem, e.g., lighting conditions and occlusions, are apparent in the figure.

contribution presented with this application is a novel use of measurements extracted through image processing to determine the behaviour of a target. The proposed solution for this application was briefly described by the author in [23, (C) 2016 The Authors. Ecology and Evolution published by John Wiley \& Sons Ltd.].

\section{Application 1.2: Tracking of Birds}

An application is considered where the times and directions of takeoffs and the amount of activity of the European robin is studied. For such experiments, the birds are placed in Emlen funnels [45] to record their movements. Traditionally, the walls are lined with scratch-sensitive paper where the scratches are counted manually after some time. The amount of activity and the average orientation of takeoffs are then estimated. The procedure of counting is often time-consuming, tedious and sensitive to the bias of the individuals performing the counting.

A proposed development of this method is to install camera surveillance of the birds during the experiments. See Figure 1.2 for an illustration of the available footage. There are mainly three advantages to this approach. Firstly, it allows for automation of the procedure, saving both time and reducing errors. Secondly, a temporal dimension is added to the data that is not available with the traditional method. Thirdly, experiments can be conducted for longer time periods. Further development of the method could potentially reduce the stress on the bird by allowing for larger cages during the experiments.

The data is generously provided by the Centre for Animal Movement Research at Lund University. All application descriptions with the word bird in the caption are related to this application. The results of the solution are presented in Application 4.1 . 


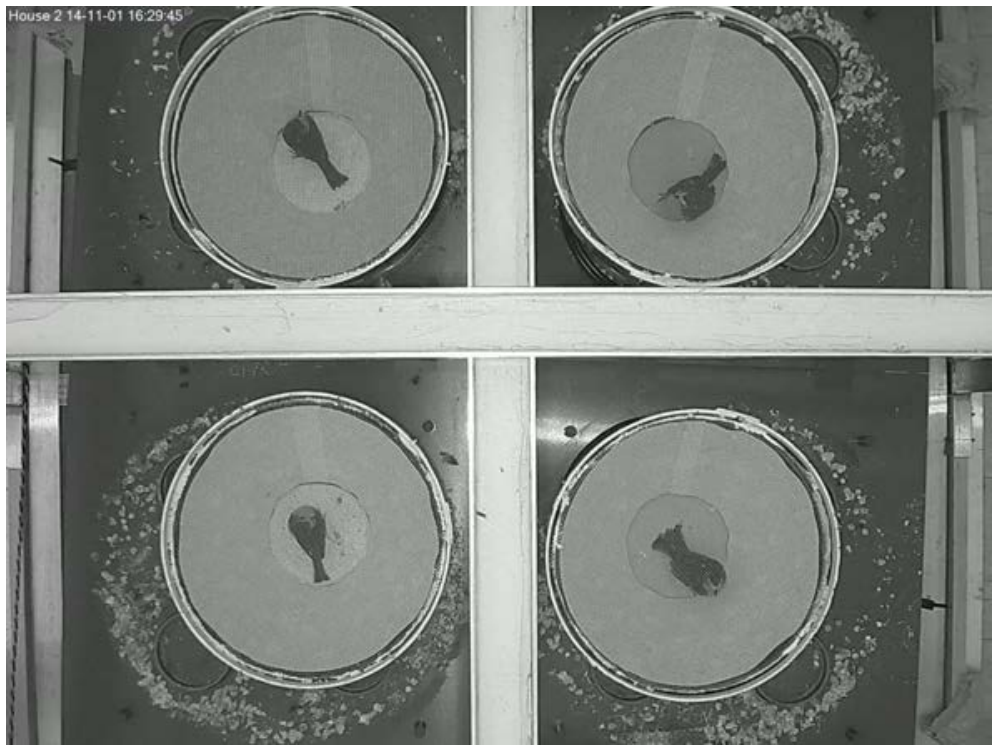

Figure 1.2: A single frame from a video recorded of the birds by a camera.

Application 1.3 introduces a scenario where the aim is to track animals, particularly rhinoceros, in a wildlife reserve in Kenya. The application is in its current state not a complete framework, but serves as an opportunity to demonstrate state-of-the-art target tracking algorithms.

\section{Application 1.3: Tracking of Savannah Animals}

A scenario is considered in this application where animals on the savannah at Kolmården Wildlife Park are recorded from a quadcopter using a traditional video camera and a thermal camera. Ultimately the goal is to classify the animals and track their absolute locations and extents in the real world. As a first attempt to find a solution, the application considers tracking the position and the extent of the animals in the image. The application also serves as a demonstration of a state-of-the-art extended target tracking algorithm.

Single frames from the video camera and thermal camera are presented in Figures 1.3 and 1.4, respectively. The two main differences between the cameras are that the video camera has a much higher resolution and field of view, and that animals are more visible in the image from the thermal camera due to their high body temperatures. Especially compare the two Lechwes that are clearly visible in the upper right hand corner in Figure 1.4, but are barely visible in Figure 1.3.

All application descriptions with the word savannah in the caption are related to this application. The results of the solution are presented in Application 5.1.

Applications 1.4 and 1.5 stem from a problem of combining measurements from rhinoceros from traditional sensors, such as radars or cameras, with observa- 


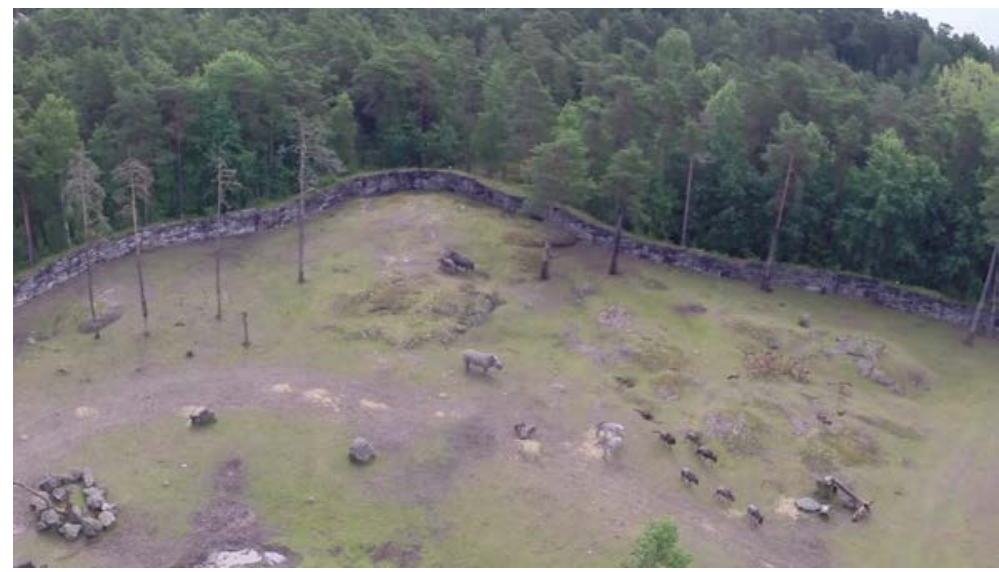

Figure 1.3: A frame recorded with a video camera over the savannah at Kolmården Wildlife Park from a quadcopter.

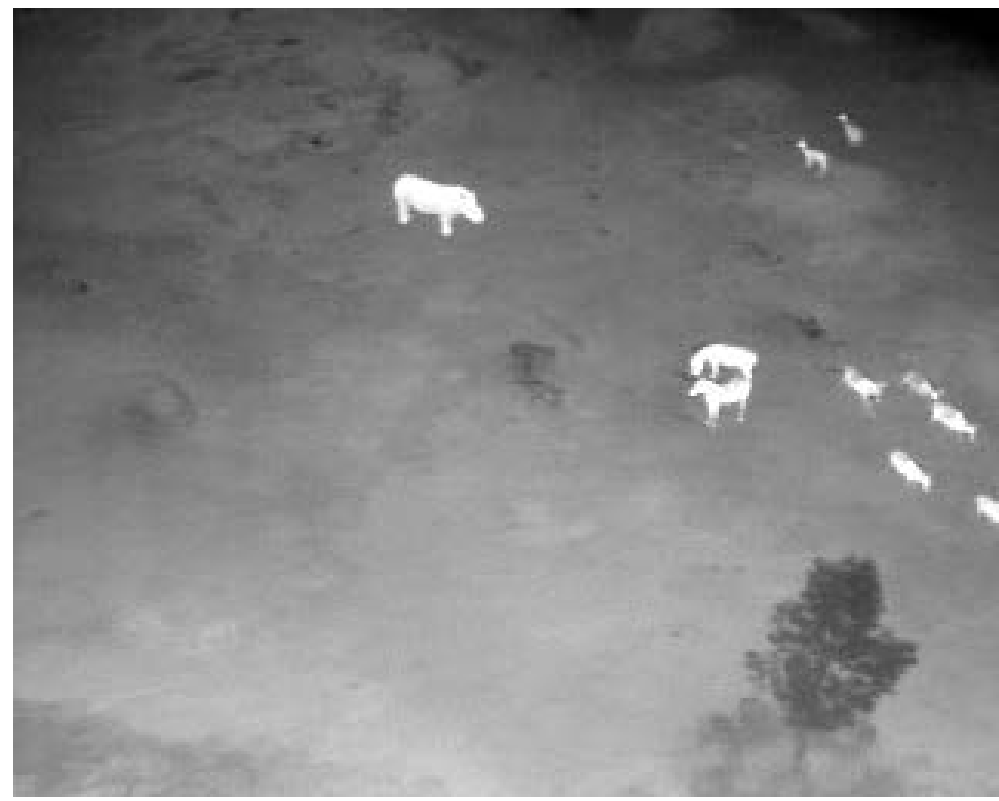

Figure 1.4: A frame recorded with a thermal camera over the savannah at Kolmården Wildlife Park from a quadcopter. 
tions from park rangers, such as browsing damage or droppings caused by the rhinoceros. The former type of measurements might be uncertain in position, but has an accurate timestamp, while the latter type of observations might be accurate in position, but has an uncertain timestamp.

Application 1.4 introduces a scenario involving a simple one-dimensional statespace model (SSM) incorporating an observation with an uncertain timestamp. The main contribution presented with this scenario is an analysis of the impact of the additional observation on the posterior distribution of the state and its estimators. The analysis and the derivations of the posterior distributions and estimators were previously published by the author in [113, (c) 2016 IEEE].

\section{Application 1.4: Simple Uncertain Time Scenario}

A simple scenario is considered in this application, where a trajectory in one dimension is measured to start at position 0 at time 0 and end in 1 at time 1 . An observation at position 0.5 is then given with a flat prior on the timestamp between 0 and 1 in time. See Figure 1.5 for an illustration of the scenario. One can of course argue that any physical quantity that starts at 0 and ends at 1 must pass through position 0.5 , so this observation seems to contain little information. This is not quite true, however, since the posterior distribution is shown to narrow down in an interesting way. The case of an observation at position 1.5 is also considered with a much more narrow prior distribution.

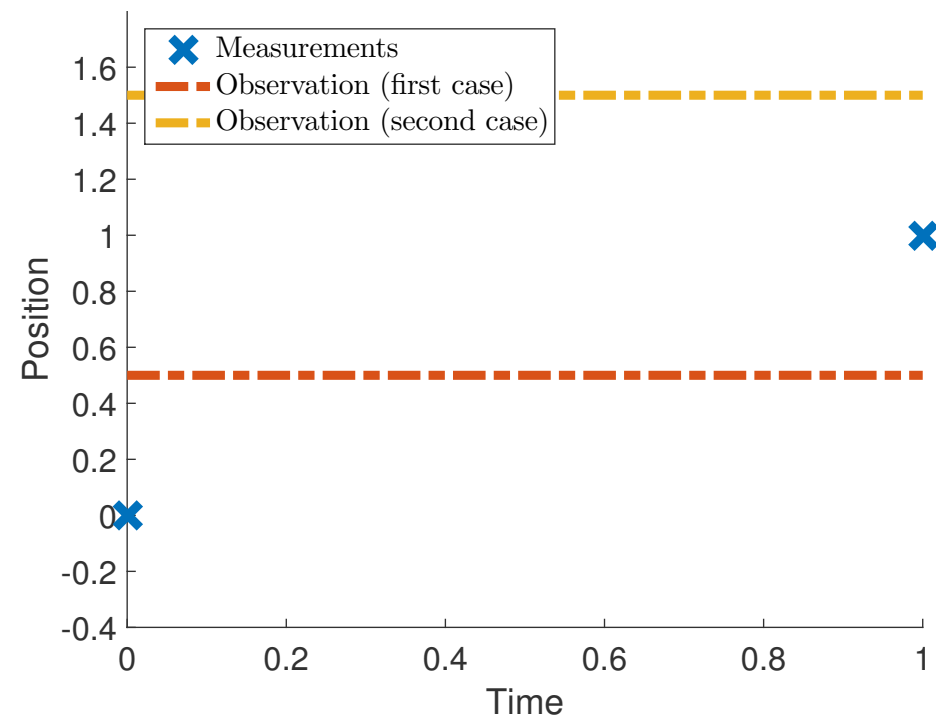

Figure 1.5: The measurements and observations for the two cases considered in the simple uncertain time scenario.

All application descriptions with the word simple in the caption are related to this application. An analysis of the results are presented in Applications 4.2, 4.3 and 4.5-4.7. 
There is no data available for the original idea for the scenario. Application 1.5 instead introduces a scenario with a corresponding problem where the aim is to improve the location estimate of an orienteering sprinter.

\section{Application 1.5: Tracking of an Orienteering Sprinter}

An application is considered where a sprinter in orienteering is passing a control in a competition, as shown in Figure 1.6. The position of the sprinter is measured using a global navigation satellite system (GNSS). In this particular section of the race the accuracy of the GNSS is poor. The location of the control is known with high accuracy and, since it is known that the sprinter passed the control, this information can be used to improve the estimated position of the sprinter.

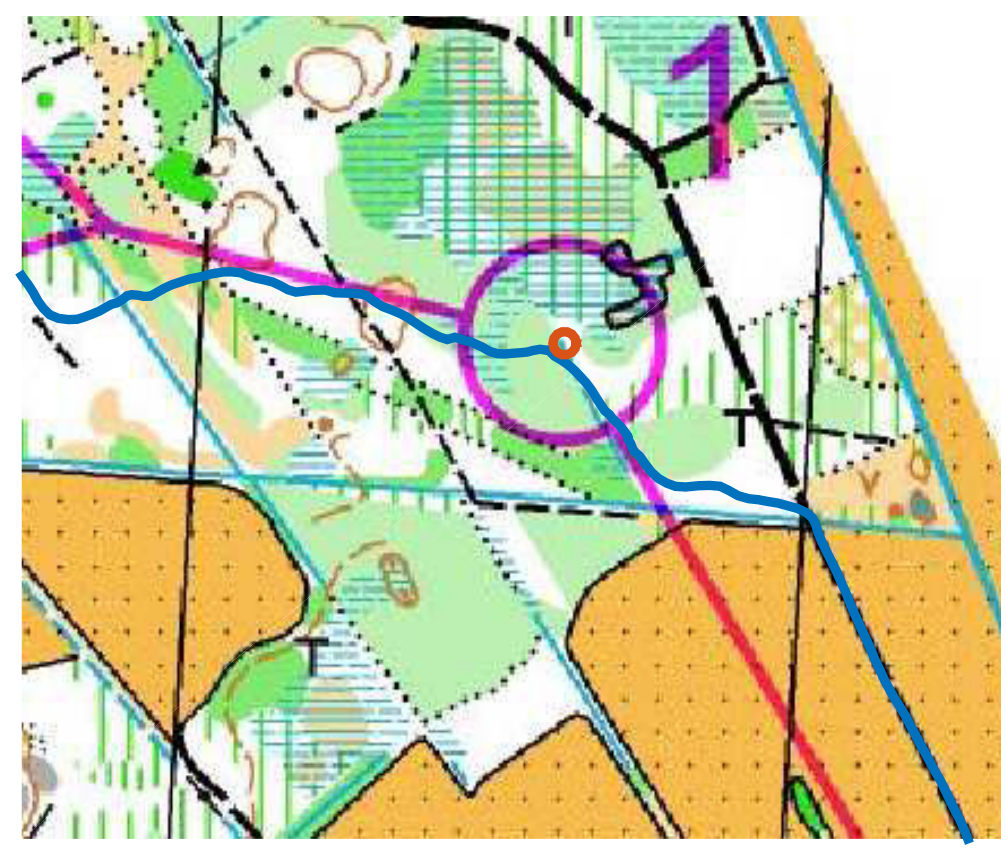

Figure 1.6: The position of a sprinter in an orienteering race as measured by a GNSS is shown in blue. The known location of a control is shown in red. Figure courtesy of OK Orion.

The data is generously provided by Oskar Ljungqvist. All application descriptions with the word orienteering in the caption are related to this application. The results of the solution are presented in Application 4.4.

\subsection{Target Tracking}

Target tracking is considered as the estimation of position, and possibly more characteristics, of an object or objects from sensor data. This involves some or all of the following steps: 


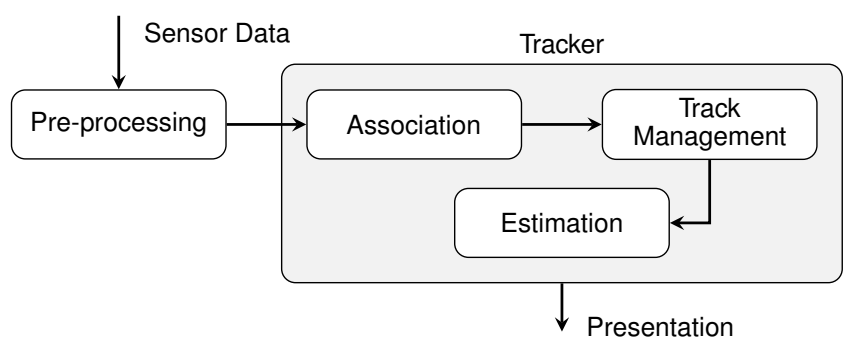

Figure 1.7: Elements of a single hypothesis tracker for MTT.

- pre-processing of sensor data to obtain detections;

- association between detections and predictions for existing tracks;

- estimation for each track using associated detections; and

- track management.

A track represents an estimate given a set of detections assumed to be generated by the same object according to a hypothesis of association.

Remark 1.1. There are no clear definitions or unique names for many of the concepts within the field, e.g., target tracking, sensor fusion, inference and estimation can often be used interchangeably as well as measurement, detection and observation. Often the meaning is given by the context, avoiding the need for a definition. This thesis is no different, but to avoid confusion, definitions will be given at times.

A target tracking framework can be presented as a pipeline of modules with welldefined interfaces, as shown in Figure 1.7. The modules have access to a central collection of tracks and hypotheses. Often there is an overlap between the modules, so a more flexible interface is needed in reality, but the pipeline is useful as an illustration of the framework.

Two common assumptions in target tracking are that a detection is generated by at most one target and that a target can generate at most one detection. If the former is violated additional logic is required and if the latter is violated extended target tracking (ETT) algorithms are needed. A nuisance common in tracking applications is that false detections occur that are not generated by a real target, commonly known as clutter. In addition, real targets possibly do not generate a detection at every time step, which is known as a missed detection.

In some applications multiple targets are considered, referred to in the literature as multiple target tracking (MTT), while in other applications it is known a priori that only a single target is present, referred to as single target tracking (STT). If only a single target is present and there are no false detections, the data association and track management steps are redundant, since only a single track needs to be considered. In all other cases the uncertainty in data association between detections and tracks needs to be considered. There is an abundance of litera- 
ture on the topic of target tracking, see e.g. $[11,12,14,24,86]$. An overview of established methods and common issues in target tracking is given in [25].

An overview of each module in the pipeline is given in the subsections. There are also other possible solutions, such as track-before-detect (TBD) [7] that performs tracking directly on raw data, or association-free algorithms, such as the probability hypothesis density (PHD) filter [115], that can be considered for some applications.

\subsubsection{Pre-processing}

The pre-processing step involves all activities required to obtain detections that can be used for association and estimation. The activities depend on the type of sensor that is used. To extract detections from camera sensors, a simple thresholding might suffice in some applications, while state-of-the-art computer vision methods might be required in other applications. A few such methods are described in Chapter 3. For radars, low-level signal processing is often used together with a threshold to produce detections. Thresholds, or similar tuning parameters, define a trade-off between true, false and missed detections. The current set of tracks can be used in feedback to this step to adaptively set the thresholds, improving the performance of the algorithm.

\subsubsection{Association}

Given detections, this step aims at finding the best associations between detections and tracks. An ambiguity in association results in multiple possible hypotheses. Simple methods, such as nearest neighbour (NN) and probabilistic data association (PDA), that work well for STT basically select the most likely detections for each track. Some more advanced methods, global nearest neighbour (GNN) and joint probabilistic data association (JPDA), designed for MTT globally finds the overall most likely association for tracks and detections, resulting in a single hypothesis. A complete solution would consider all possible associations in different hypotheses and select the currently most likely hypothesis. One such MTT solution is the multiple hypothesis tracker (MHT). However, maintaining all hypotheses over time is computationally demanding, so clever approximations are used that only maintain a set of the most likely hypotheses.

Each hypothesis generated in the association step consists of a set of tracks where the associations with detections are assumed to be certain and estimation can be performed. In addition to associating detections to existing tracks, some may also be identified as false detections or new tracks, requiring additional logic. To improve performance and to avoid unlikely associations a gate is used for each track to only consider detections that are likely. The methods are described in depth in [25] and its references, and some aspects and methods for data association are covered in Chapter 5. 


\subsubsection{Estimation}

Estimation is performed for each track in each hypothesis, where the set of detections associated to the tracks are given. There is an abundance of literature on this topic that goes beyond the problem of target tracking, see e.g. $[4,61,63,76,101]$.

The main idea is to use an SSM to describe the dynamics of the target and model the sensors, where the states are estimated using measurements from the sensors. Estimators for SSMs are commonly divided into

- filter, when data is available up to current time;

- predictor, when data is available up to a previous time; and

- smoother, when data is available up to a future time.

The most notable method is the Kalman filter (KF) [74], which for the linear Gaussian state-space model (LGSSM) gives the exact solution in closed form. A corresponding smoother algorithm is the Rauch-Tung-Striebel smoother (RTSS) [97]. For nonlinear or non-Gaussian models an approximation, such as the extended Kalman filter (EKF) [105] or unscented Kalman filter (UKF) [72, 73], can be used or a sequential Monte Carlo method, such as the particle filter (PF) [58]. When the type of target being tracked is unknown or the target behaviour is expected to change, a jump Markov model (JMM) [109] is suitable, for which a set of filters or smoothers can be used to determine an appropriate model. Common estimation methods for such models are the filter bank [6] and the interacting multiple model (IMM) filter [26]. SSMs are covered in Chapter 2 and methods for statespace estimation are covered in Chapter 4.

\subsubsection{Track Management}

Track management is performed to initiate, merge and terminate tracks, and for MHT also hypothesis management is considered.

Unassociated detections are used to initiate new tracks, called initiators or tentative tracks. A track is confirmed if a sufficient number of measurements has been associated with the initiator, determined by a track score or initiator logic. In some methods two layers of data association are performed, first for confirmed tracks and then for initiators, in order to guarantee a priority for confirmed tracks. A track is deleted if a sufficient number of missed detections has occurred, determined by the track score or a deletion logic. For low-resolution sensors, entire groups of targets might belong to a single track, and additional logic is often required to handle merging and splitting of such tracks when targets enter or leave the group. In hypothesis-oriented MHT, initiators are treated differently, where management is performed on the hypothesis level. It is intractable to consider all possible hypotheses, so a set of the most likely hypotheses is maintained between iterations, pruning unlikely hypotheses. The methods are described in detail in [25] and some track management policies are covered in Chapter 5. 


\subsection{Motivation and Background}

Target tracking is covered in this thesis. Select established methods are presented and some particular problems within target tracking are covered in more detail. Some minor developments are described and solutions to three main problems are proposed.

The first problem is when the observations suffer from uncertainty in the sampling times. The problem of uncertain sampling time seems less studied in literature, with out-of-sequence (OOS) measurements and sampling time jitter being notable exceptions. A discrete-time linear Gaussian state-space model is considered in this thesis for a single observation with an uncertain sampling time.

The second problem is when targets have additional conditions on the state vector, which traditionally is assumed to be unconstrained in the real coordinate space. Conventional motion models do not satisfy such constraints and produce infeasible predictions. The particular case considered is a target position that is constrained to be within a region. One option is to project the state back into the region. Another approach is considered in this thesis where the motion model is adapted to the region to produce feasible predictions.

The third problem is how to incorporate measurements that are directly dependent on the mode into a JMM. For traditional JMMs the SSM parameters are dependent on the mode, resulting in an indirect effect on the measurement. The case considered in this thesis is that some measurements are observations of an arbitrary distribution that directly depends on the mode.

Biologists spend a significant part of their time collecting and analysing animal data. The manual methods are prone to bias and the time could perhaps be better spent on other research activities. Target tracking can provide a solution to this problem by automating the procedures of collecting and analysing data.

Many endangered animal populations in the world are still on the decline. There are numerous underlying causes for this, but poaching is a significant reason in many wildlife reserves. Park rangers often have scarce resources and would benefit from cost-effective solutions providing situational awareness, which would allow for improved coordination of conservation efforts. This could be provided by incorporating various sensors, such as ground and airborne cameras, smart phones, and radars, into a single network for tracking animals. Such ambitions are found in the project Smart Savannahs [104] related to this research.

Providing biologists and park rangers with complete solutions for the problems they face is a rather ambitious goal. Instead, steps on the way are presented in the form of methods to approach particular problems within target tracking. Solutions for a few applications are presented with clear ambitions towards aiding biologists and park rangers. However, the main interest is in the technical challenges posed by the problems within the applications and the technical solutions to those problems. 
Table 1.1: List of theory and features that are exemplified by the different applications in Section 1.1.

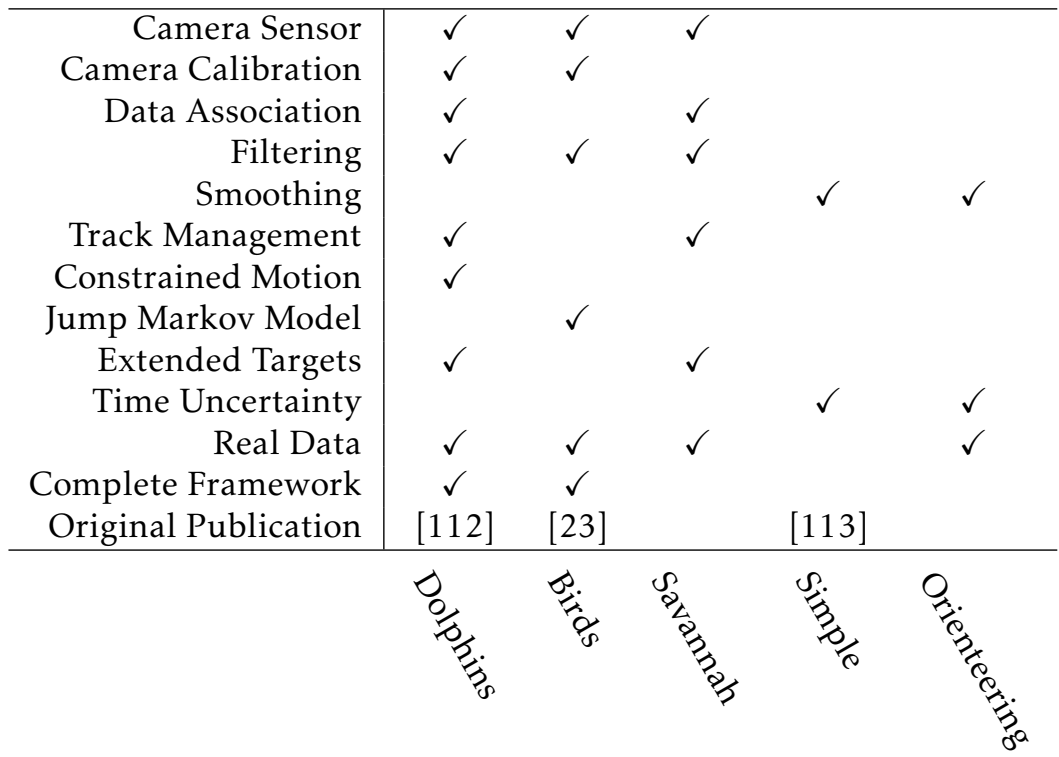

Motivation and background for the individual applications introduced in Section 1.1 are covered in the following subsections. Table 1.1 is provided as a reference for the features of the solutions and the theory required for each application.

\subsubsection{Dolphin Application}

A recent project for tracking dolphins in a dolphinarium, described in [75], was set up at Brookfield Zoo in Chicago. The researchers utilised multiple overhead and underwater cameras to record data for over a year. Due to the massive amounts of data, manual tracking was only attempted for a small portion of the data and from a single view. The article describes a method of using robust principal component analysis [33] and Gaussian-mixture background modelling [51] to detect the dolphins and a compressive tracker [122] to extract trajectories. The trajectories were compared to the manually annotated data with promising results.

There have also been some attempts to track dolphins in the wild to study their behaviour, e.g. in [52], where boat-side overhead cameras were used, and in [67], where underwater cameras and hydrophones were used together. Another project related to this research is described in [27], where a large number of underwater cameras over the duration of three years recorded fish at a coral reef. The purpose was, however, to classify the species of the fish rather than to track their location, but it demonstrates the growing importance of camera surveillance in animal behaviour studies. 
In the dolphin application introduced in Application 1.1, the goal is to construct a complete target tracking solution from camera to trajectories. To achieve this goal methods for image processing, camera calibration, target tracking and modelling of dynamic systems are used. In addition, new methods for the complete solution are required for:

- using more information from background estimation for tracking;

- modelling a camera without the possibility of a proper calibration;

- data reduction and mapping of a large number of detections;

- modelling dolphin motion in captivity; and

- object extension estimation.

\subsubsection{Bird Application}

Bird migration has fascinated biologists for centuries. The birds follow a migratory program inherited from their parents during their first migration. The program encodes durations and directions to fly, and to navigate the birds rely on biological compasses based on the sun, stars and magnetic fields [65]. A method in which migratory birds are kept in funnel-shaped cages was introduced half a century ago in [45]. The cages, called Emlen funnels, record scratches from the birds with ink or thermal paper as they attempt to take off and allow biologists to study the compass mechanisms of migratory birds. Several results from studies involving Emlen funnels have been published, e.g. in [44] and [120].

The Emlen funnel method is primarily manual and has remained largely unchanged in the last few decades. Some attempts at automating the process using electronic switches to detect movement, e.g. in [118], have shown not to improve the method and in addition suffered interference from electromagnetic noise. However, passive cameras mounted at a distance from the birds, used in [88], do not cause such interferences. Recently, software has also been made available, presented in [89], for evaluating activity and orientations of the birds, showing a trend to modernise the traditional method.

In the bird application introduced in Application 1.2, the goal is to estimate the activity and the takeoff times and directions of birds from a recorded video. To achieve this goal methods for camera calibration, JMMs and estimation for such models are used. In addition, new methods for the complete solution are required for:

- modelling a camera without the possibility of a proper calibration;

- modelling modes in a JMM;

- estimating times of takeoffs; and

- estimating direction of takeoffs. 


\subsubsection{Savanna Application}

In the last few decades, the market for commercially available low-cost small unmanned aerial systems has exploded. Flight time is still rather limited and in most cases technical skills are required to set up and operate the unmanned aerial systems. However, they provide new opportunities for solving traditionally tedious, difficult or dangerous problems. One promising area for deploying unmanned aerial vehicles is in surveillance of large land masses, such as wildlife reserves, to count and track wild animals for conservation purposes. Wildlife is traditionally monitored by foot, satellite or manned aircraft, with high costs as a result.

As a first attempt, many projects using unmanned aerial systems essentially put a camera in the sky, allowing conservationists to count and track animals manually in the video, see e.g. $[69,70,114]$ for a small selection of many such projects. Other projects are more ambitious and in addition to deploying unmanned aerial systems perform image processing for automatic detection and counting of animals, see e.g. $[56,87,110,119]$ for a selection of such projects. A project targeted directly at anti-poaching of rhinoceros is presented in [90], where an unmanned aerial system was deployed in the KwaZulu-Natal province in South Africa. The article discusses the technical difficulties involved, advantages and disadvantages of the approach as well as the ethical and legal aspects. A project for tracking animals in captivity from an aerial cableway through Kolmården Wildlife Park is presented in [47]. The long time between detections seemed to be an issue for maintaining tracks.

In the savannah application introduced in Application 1.3, the ultimate goal is to track the position and extension of savannah animals in real-world coordinates using an airborne camera as well as to classify the targets. As a stepping stone, a lower goal is set in this thesis to track the positions and extensions of the savannah animals in image coordinates. To achieve this, methods for background estimation and state-of-the-art ETT are used.

\subsubsection{Uncertain Time Scenarios}

Traditionally in target estimation, the information obtained is assumed to be noise, while the sampling time is assumed to be known accurately. However, there are many applications where some information is uncertain in time. One such application involving traces of rhinoceros is proposed earlier in this chapter. Another potential application is in crime scene investigations, where the place of the crime often is known accurately, but not the time, and witness statements often contain uncertainty in both time and place, while surveillance cameras are precise in time.

Parameter and state estimation in dynamical models with uncertain and stochastic timestamps is to the author's knowledge a less studied problem in literature. Sampling jitter is one exception, see for instance [46], where estimation problems are formulated based on parametric models mixed with prior distributions of the 
sampling times. However, this idea has seemingly not been explored in target tracking.

One attempt would be to consider the uncertain timestamps as latent variables and use for example the expectation-maximization (EM) algorithm [40]. For instance, [102] discusses missing data in an SSM, using the EM algorithm, and this is further developed in, e.g., [68] and [92]. Further, [54] discusses using the EM algorithm to estimate synchronization. The latter is related to timing issues, but the author has not found any study directly related to the problem formulation.

One can see observations with an uncertain timestamp as oos measurements. As presented in literature, these have precise timestamps, but are available at a later time than the actual measurement, and have to be processed in a smoothing framework. Theory on this topic is developed in [10] and [121]. In this context, [8] discusses acceleration bias caused by synchronization issues between a GNSS and an inertial measurement unit (IMU), which is further elaborated on in [103]. Further, [94] discusses time delays caused by wave propagation.

For the simple uncertain time scenario introduced in Application 1.4, the goal is to obtain posterior distributions and estimators for a one-dimensional LGSSM with an observation sampled at an uncertain time. To achieve this goal methods are used for smoothing in an SSM and for point estimation of mixture distributions. In addition, new methods are required for

- modelling of an observation with an uncertain timestamp in an SSM; and

- computing smoothing posterior distributions for such models.

For the orienteering application introduced in Application 1.5, the ultimate goal is to improve the location estimate of a sprinter using control points as observations with uncertain timestamps. In this thesis the goal is to consider one such observation. To achieve this goal, the same methods as for the simple uncertain time scenario are required, but they need to be applied in two dimensions.

\subsection{Contributions and Publications}

There are three main contributions in this thesis:

1. The development and analysis of a model with an observation sampled at an uncertain time.

2. The development of a constrained motion model.

3. The development of an extension to the JMM incorporating state-independent measurements.

The first contribution has previously been published in a paper on models with an observation sampled at an uncertain time,

C. Veibäck, G. Hendeby, and F. Gustafsson. On fusion of sensor measurements and observation with uncertain timestamp for target track- 
ing. In Proceedings of the 19th International Conference on Information Fusion, pages 1268-1275, Heidelberg, Germany, July 2016. (C) 2016 IEEE.

All examples in this thesis concerning the simple uncertain time scenario are also presented in the paper. In addition, the paper presents methods developed by the author on

- modelling with observations sampled at an uncertain time, restated in Section 2.7; and

- estimation for models with observations sampled at an uncertain time, restated in Section 4.6;

The content is reused in this thesis courtesy of IEEE. The author's contribution to the paper was in implementation of algorithms, theoretical derivations and writing the majority of the manuscript. Co-authors aided plenty in discussions and input as well as in part writing the manuscript.

The second contribution has previously been published in a paper on a tracking solution for dolphins in captivity,

C. Veibäck, G. Hendeby, and F. Gustafsson. Tracking of dolphins in a basin using a constrained motion model. In Proceedings of the 18th International Conference on Information Fusion, pages 1330-1337, Washington D. C., USA, July 2015. C 2015 IEEE.

All examples in this thesis concerning the dolphin application are also presented in the paper. In addition, the paper presents methods developed or improved by the author on

- constrained motion models, restated in Section 2.3;

- camera sensors, foreground segmentation and data reduction, restated in Chapter 3; and

- data association, restated in Section 5.3.3.

The content is reused in this thesis courtesy of IEEE. The author's contribution to the paper involves implementation of algorithms, theoretical derivations and writing the majority of the manuscript. Co-authors aided plenty in discussions and input as well as in part writing the manuscript.

The third contribution has in part been published in a paper on updated methods for studying compass orientation in songbirds,

G. Bianco, M. Ilieva, C. Veibäck, K. Öfjäll, A. Gadomska, G. Hendeby, M. Felsberg, F. Gustafsson, and S. Åkesson. Emlen-funnel experiments revisited: methods update for studying compass orientation in songbirds. Ecology and Evolution, 6(19):6930-6942, 2016. ISSN 20457758. doi:10.1002/ece3.2383. (c) 2016 The Authors. Ecology and Evolution published by John Wiley \& Sons Ltd. 
Some of the examples in this thesis concerning the bird application are also presented in the paper. In addition, the appendix to the paper presents methods developed by the author on:

- extending the JMM to incorporate state-independent measurements, restated in Section 2.5.2; and

- estimation for the JMM extended with state-independent measurements, restated in Section 4.4.2;

The author's contribution to the paper was in deriving and implementing the filter in the Computer vision method, providing results from the method to the first author and in writing the parts of the manuscript and appendix related to the filter.

In addition, the author has published two papers that are not directly related to the content of this thesis. The first paper, on a bounded confidence model, is

F. Ceragioli, G. Lindmark, C. Veibäck, N. Wahlström, M. Lindfors, and C. Altafini. A bounded confidence model that preserves the signs of the opinions. In Proceedings of the 2016 European Control Conference, Aalborg, Denmark, June 2016,

and the second paper, on education in industrial control systems, is

S. Gunnarsson, Y. Jung, C. Veibäck, and T. Glad. IO (implement and operate) first - an alternative way to approach the automatic control subject. In Proceedings of 5:e Utvecklingskonferensen för Sveriges ingenjörsutbildningar, Uppsala, Sweden, Feb. 2016.

The author's contribution to the first paper was in initial derivations and simulations for the model, performed as an exercise in a course on the subject by Prof. Claudio Altafini. The author's contribution to the second paper was in discussions on the content with the first author of the paper after having taught in the course.

\subsection{Thesis Outline}

Four applications and one simulated scenario are used throughout the thesis to demonstrate theory in practice. The applications and a framework for target tracking are introduced in Chapter 1.

A target exhibits certain behaviours that are captured in a dynamic model and observations of targets are captured in a measurement model, which together result in an SSM. Representations of SSMs, common dynamic and measurement models and some models tailored to particular applications are covered in Chapter 2.

Cameras are used as sensors in three of the applications in this thesis. A significant amount of pre-processing is often required and the nonlinearity of the 
sensors is often non-trivial. Methods for modelling camera sensors as well as some image processing methods are covered in Chapter 3.

The main goal is to infer information from available measurements using a model of a system. For an SSM this is referred to as state-space estimation, where the states are estimated. Estimation of other unknown parameters are also of interest for some models. Select methods for estimation are covered in Chapter 4.

The observation origins are often uncertain in target tracking. Estimation for such scenarios often leads to an intractable problem and various methods for handling the uncertainty are required. A few methods are covered in Chapter 5.

Conclusions are drawn and future work is discussed in Chapter 6. 


\section{2}

\section{State-Space Models}

A state-space model (SSM) is a mathematical model of a system where all necessary information required to predict the system behaviour is condensed into a state vector [5]. In essence, an SSM is a system of first-order differential or difference equations representing the dynamics of the system together with a measurement equation. Often the dynamics of the underlying system is continuous in time. However, observations in computerised systems are obtained in discrete time and the state can only be represented in a finite number of time steps. A common approach is therefore to initially model the dynamics in continuous time and then discretise the model to allow for inference.

Real systems are rarely deterministic, but exhibit random behaviour that cannot be predicted deterministically. This is modelled using a stochastic SSM, where stochastic is implied in the sequel, obtained by introducing stochastic variables into the SSM [5]. Although it is possible to model randomness in continuous time, stochastic variables will only be considered in discrete time in this thesis.

The outline of the chapter is as follows: A few structures and representations of the discrete-time SSM are introduced in Section 2.1. Some common dynamic models are described in Section 2.2. In Section 2.3 a motion model for targets constrained to a region is derived. The model was previously presented by the author in [112]. Sensor models are outlined in Section 2.4 and in Section 2.5 the JMM is described and an extension is proposed by the author. The extension to the model was previously presented by the author in [23]. A model for extended targets is described in Section 2.6. In Section 2.7 a variation of the SSM is presented for systems where an observation has an uncertain timestamp, which was previously presented by the author in [113]. 


\subsection{General Description}

An SSM can be represented in a number of ways and a few useful representations are presented in this section. Introductions to SSMs in a stochastic framework are given in, e.g., [5] and [82].

The most general model description of a system considered is the probabilistic state-space model (PSSM) [101], where the probability density functions (PDF) are modelled directly. The PSSM is defined as

$$
\begin{aligned}
& \mathbf{x}_{0} \sim p\left(\mathbf{x}_{0}\right), \\
& \mathbf{x}_{k} \sim p\left(\mathbf{x}_{k} \mid \mathbf{x}_{k-1}\right), \\
& k \in \mathcal{K}=\{1: N\}, \\
& \mathbf{y}_{j} \sim p\left(\mathbf{y}_{j} \mid \mathbf{x}_{j}\right) \text {, } \\
& j \in \mathcal{J} \subseteq \mathcal{K} \text {, }
\end{aligned}
$$

where

- $\mathbf{x}_{k} \in \mathbb{R}^{n}$ is the state vector at time $t_{k}$;

- $\mathbf{y}_{j} \in \mathbb{R}^{m}$ is an observation of the state vector at time $t_{j}$;

- $p\left(\mathbf{x}_{k} \mid \mathbf{x}_{k-1}\right)$ is the dynamic model of the system represented as a PDF;

- $p\left(\mathbf{y}_{j} \mid \mathbf{x}_{j}\right)$ is the observation model of the system represented as a PDF; and

- $p\left(\mathbf{x}_{0}\right)$ is the PDF for the initial state.

The dynamic and observation models are assumed to be Markovian in the sense that

$$
\begin{gathered}
p\left(\mathbf{x}_{k} \mid \mathcal{X}_{1: k-1}, \mathcal{Y}_{1: k-1}\right)=p\left(\mathbf{x}_{k} \mid \mathbf{x}_{k-1}\right), \\
p\left(\mathbf{y}_{j} \mid \mathcal{X}_{1: j}, \mathcal{Y}_{1: j-1}\right)=p\left(\mathbf{y}_{j} \mid \mathbf{x}_{j}\right),
\end{gathered}
$$

where $\mathcal{X}_{1: a}=\left\{\mathbf{x}_{k}\right\}_{k \in\{1: a\}}$ and $\mathcal{Y}_{1: a}=\left\{\mathbf{y}_{j}\right\}_{j \in\{1: a\} \cap \mathcal{J}}$ are the sets of all states and measurements, respectively, up to time $a$. When $\mathcal{J}=\mathcal{K}$ or there is no ambiguity, index $k$ is sometimes used instead of $j$ for measurements.

This model structure is convenient for deriving the posterior distribution, but often it is simpler to model a system on the slightly more restrictive structure of the nonlinear state-space model (NSSM) [5].

The NSSM is defined as the PSSM with the constraint that

$$
\begin{aligned}
\mathbf{x}_{k} & =\mathbf{f}_{k}\left(\mathbf{x}_{k-1}, \mathbf{w}_{k}\right), \\
\mathbf{y}_{j} & =\mathbf{h}_{j}\left(\mathbf{x}_{j}, \mathbf{v}_{j}\right),
\end{aligned}
$$

where transition function $\mathbf{f}_{k}(\mathbf{x}, \mathbf{w})$ and measurement function $\mathbf{h}_{j}(\mathbf{x}, \mathbf{v})$ are arbitrary nonlinear functions, often with the time index omitted, and

$$
\begin{aligned}
\mathbf{w}_{k} & \sim p\left(\mathbf{w}_{k}\right), \\
\mathbf{v}_{j} & \sim p\left(\mathbf{v}_{j}\right) .
\end{aligned}
$$

A common special case of the NSSM is when the dynamic model is a nonlinear 
function of the state and white Gaussian noise enters the model additively, resulting in the additive white Gaussian noise state-space model (AWGNSSM).

The AWGNSSM is defined as the NSSM with certain constraints. The additive noise constraint on the SSM is that

$$
\begin{aligned}
& \mathbf{x}_{k}=\mathbf{f}_{k}\left(\mathbf{x}_{k-1}\right)+\mathbf{w}_{k}, \\
& \mathbf{y}_{j}=\mathbf{h}_{j}\left(\mathbf{x}_{j}\right)+\mathbf{v}_{j} .
\end{aligned}
$$

The white Gaussian noise constraint on the noise distributions is that

$$
\begin{aligned}
\mathbf{w}_{k} & \sim \mathcal{N}\left(\mathbf{0}, \mathbf{Q}_{k}\right), \\
\mathbf{v}_{j} & \sim \mathcal{N}\left(\mathbf{0}, \mathbf{R}_{j}\right)
\end{aligned}
$$

and on the initial state that

$$
\mathbf{x}_{0} \sim \mathcal{N}\left(\overline{\mathbf{x}}_{0}, \mathbf{P}_{0}\right),
$$

where $\mathbf{Q}_{k} \in S_{+}^{n}, \mathbf{R}_{j} \in S_{+}^{m}$ and $\mathbf{P}_{0} \in S_{++}^{n}$.

The most important special case is obtained by further assuming the functions $\mathbf{f}_{k}(\mathbf{x})$ and $\mathbf{h}_{j}(\mathbf{x})$ in the AWGNSSM to be linear, resulting in the linear Gaussian state-space model (LGSSM) [5]. The reason for its importance is that since the initial state is Gaussian, the posterior distributions of all subsequent states and predicted measurements are also Gaussian, which simplifies inference in the model considerably.

The LGSSM is defined as the AWGNSSM with the constraint that

$$
\begin{aligned}
& \mathbf{x}_{k}=\mathbf{F}_{k} \mathbf{x}_{k-1}+\mathbf{w}_{k}, \\
& \mathbf{y}_{j}=\mathbf{H}_{j} \mathbf{x}_{j}+\mathbf{v}_{j},
\end{aligned}
$$

where the transition matrix $\mathbf{F}_{k}$ and the measurement matrix $\mathbf{H}_{j}$ are assumed to be independent of the state $\mathbf{x}_{k}$.

Generally the transition functions (2.3a), (2.5a) and (2.8a) are functions of the discretisation time $T_{k}=t_{k}-t_{k-1}$, where, for most models considered, the discretisation time is constant and denoted $T_{k}=T$.

\subsection{Motion Models}

In target tracking applications, the state vector commonly represents the position and motion of the target being tracked, so $\mathbf{f}_{k}(\mathbf{x}, \mathbf{w})$ in $(2.3 \mathrm{a})$ is therefore referred to as a motion model. The model can be tailored to a particular type of target or application, or a suitable general motion model can be tuned to fit the application. See [81] for a selection of common motion models.

It is often difficult to model the target accurately. If the target is intelligent, e.g., if the target is a human, an animal or a vehicle controlled by a human, the intelligent part of the behaviour must often be modelled as noise. Sometimes one can make assumptions on constraints on the motion of the expected target type, e.g., 
a fixed wing aircraft has limited turning radius and cars generally stay on roads, which can be encoded into the model.

First, the dimension $p$ of the position of the target is determined. For motion in a plane, such as on the ground, a two-dimensional model is often sufficient, while for aerial targets motion in all three dimensions is possible. The first states of the state vector in a motion model are chosen as the $p$-dimensional position.

The simplest model is to assume that the position is nearly constant, with a zeromean stochastic velocity, commonly referred to as a random walk model, constant position model or nearly constant position model (NCPM) [81]. The NCPM is a linear Gaussian motion model on the form (2.8a) with

$$
\mathbf{F}_{k}=\mathbf{I}_{p} \quad \text { and } \quad \mathbf{Q}_{k}=\mathbf{B}_{k} \tilde{\mathbf{Q}}_{k} \mathbf{B}_{k}^{T},
$$

where $\tilde{\mathbf{Q}}_{k} \in S_{+}^{p}, \mathbf{B}_{k}=T_{k} \otimes \mathbf{I}_{p}$ and $\otimes$ is the Kronecker product. The state vector is modelled by the position, which for two dimensions and omitted time index is given by

$$
\mathbf{x}=\left(\begin{array}{ll}
x & y
\end{array}\right)^{T} .
$$

A slightly more complex, but often useful, model is obtained by assuming that the velocity is nearly constant, commonly referred to as a constant velocity model or a nearly constant velocity model (NCVM) [81]. The NCVM can be seen as an extension of the NCPM where the state vector has been augmented by the velocity, which for two dimensions and omitted time index becomes

$$
\mathbf{x}=\left(\begin{array}{llll}
x & y & \dot{x} & \dot{y}
\end{array}\right)^{T},
$$

with a motion model given by

$$
\mathbf{F}_{k}=\left(\begin{array}{cc}
1 & T_{k} \\
0 & 1
\end{array}\right) \otimes \mathbf{I}_{p} \quad \text { and } \quad \mathbf{Q}_{k}=\mathbf{B}_{k} \tilde{\mathbf{Q}}_{k} \mathbf{B}_{k}^{T},
$$

where

$$
\mathbf{B}_{k}=\left(\begin{array}{c}
\frac{T_{k}^{2}}{2} \\
T_{k}
\end{array}\right) \otimes \mathbf{I}_{p} .
$$

Another conventional motion model is the coordinated turn model (CTM) [81], where the speed and turning rate are assumed to be nearly constant. The CTM can be seen as an extension of the NCVM where the state vector has been augmented by the turning rate, which for two dimensions and omitted time index becomes

$$
\mathbf{x}=\left(\begin{array}{lllll}
x & y & \dot{x} & \dot{y} & \omega
\end{array}\right)^{T} .
$$

The CTM is not linear, however, but is an additive white Gaussian noise motion 
model on the form (2.5a) with

$$
\mathbf{f}_{k}\left(\mathbf{x}_{k}\right)=\left(\begin{array}{c}
x_{k}+\frac{\dot{x}_{k}}{\omega_{k}} \sin \left(\omega_{k} T_{k}\right)-\frac{\dot{y}_{k}}{\omega_{k}}\left(1-\cos \left(\omega_{k} T_{k}\right)\right) \\
y_{k}+\frac{\dot{x}_{k}}{\omega_{k}}\left(1-\cos \left(\omega_{k} T_{k}\right)\right)+\frac{\dot{y}_{k}}{\omega_{k}} \sin \left(\omega_{k} T_{k}\right) \\
\dot{x}_{k} \cos \left(\omega_{k} T_{k}\right)-\dot{y}_{k} \sin \left(\omega_{k} T_{k}\right) \\
\dot{x}_{k} \sin \left(\omega_{k} T_{k}\right)+\dot{y}_{k} \cos \left(\omega_{k} T_{k}\right) \\
\omega_{k}
\end{array}\right),
$$

where the process noise for the last state is independent from the process noise of the other states.

In Application 2.1 the motion of the bird is modelled using an NCPM with a twodimensional position.

\section{Application 2.1: Bird Motion Model}

The frame rate of the video is low and the volume within the funnel is small, making an NCPM sufficient. Although the funnel is three-dimensional, it is impossible with a single camera angle to reliably observe the bird's vertical position. The choice therefore lands on tracking the bird's position in two dimensions and the state vector is chosen as

$$
\mathbf{x}_{k}=\left(\begin{array}{l}
x_{k} \\
y_{k}
\end{array}\right)
$$

where $x_{k}$ is the bird's position in the magnetic east direction, and $y_{k}$ is the position in the magnetic north direction. The motion model is

$$
\mathbf{x}_{k}=\mathbf{x}_{k-1}+\mathbf{w}_{k},
$$

where $\mathbf{w}_{k} \sim \mathcal{N}\left(\mathbf{0}, T^{2} \tilde{\mathbf{Q}}\right), T$ is the inverse of the frame rate, and $\tilde{\mathbf{Q}}=\sigma_{w}^{2} \mathbf{I}$ determines the amount of movement of the bird.

In Application 2.2 the sprinter in the orienteering application is modelled using an NCVM with a two-dimensional position.

\section{Application 2.2: Orienteering Motion Model}

Without any information about the terrain that the sprinters move in, a reasonable assumption is that the sprinters take the shortest path at a stable pace to the next control. The sprinters are also assumed to remain on the ground, which is assumed to be flat. A two-dimensional NCVM is therefore suitable for the sprinters. The motion model is then given by

$$
\mathbf{x}_{k+1}=\mathbf{F} \mathbf{x}_{k}+\mathbf{w}_{k},
$$

where the state vector is

$$
\mathbf{x}_{k}=\left(\begin{array}{llll}
x_{k} & y_{k} & \dot{x}_{k} & \dot{y}_{k}
\end{array}\right)^{T},
$$


the transition matrix for the motion model is

$$
\mathbf{F}=\left(\begin{array}{llll}
1 & 0 & T & 0 \\
0 & 1 & 0 & T \\
0 & 0 & 1 & 0 \\
0 & 0 & 0 & 1
\end{array}\right),
$$

and the process noise $\mathbf{w}_{k} \sim \mathcal{N}(\mathbf{0}, \mathbf{Q})$ has the covariance

$$
\mathbf{Q}=\left(\begin{array}{cccc}
T^{4} / 4 & 0 & T^{3} / 2 & 0 \\
0 & T^{4} / 4 & 0 & T^{3} / 2 \\
T^{3} / 2 & 0 & T^{2} & 0 \\
0 & T^{3} / 2 & 0 & T^{2}
\end{array}\right) \sigma_{w}^{2},
$$

where $T$ is the GPS sampling time, and $\sigma_{w}^{2}$ determines how well the NCVM models the sprinter.

\subsection{Constrained Motion Model}

Conventional motion models describe the relationship between the states over time, but do not include constraints on the values of the states. These models work well for many targets that can move freely. If the target is constrained to a particular region, it is possible to explicitly define constraints, but estimation for the model would result in a constrained optimization problem. An alternative approach is to design a model where predictions of the target remain inside the constrained region. One such model, denoted as a constrained motion model (CMM), is developed by the author in [112] and is restated in this section.

\subsubsection{Motivation}

When a target is constrained to a region, adapting the motion model to reflect this can improve the tracking performance. In the following, this is achieved by making a few assumptions, listed in Assumption A2.1, about the target behaviour close to the boundary of the region. The inspiration comes from research on obstacle avoidance using potential fields [77].

Assumption A2.1. The assumptions made about the target motion are the following:

1. The target moves in two dimensions.

2. The target speed is nearly constant.

3. The target avoids moving into the boundary by turning when it gets close.

4. The target aligns its velocity with the tangent of the boundary by turning when close to the boundary. 
5. The target moves either in a clockwise or counter-clockwise direction along the boundary of the region, resulting in a preferred turning direction when avoiding collision and aligning with the boundary.

6. The strength of the influence from each point along the boundary is assumed to be a function of the state of the target and the point.

The state of the target is modelled with position $\mathbf{p}$ and velocity $\dot{\mathbf{p}}$ in two dimensions as

$$
\mathbf{x}=\left(\begin{array}{c}
\mathbf{p} \\
\dot{\mathbf{p}}
\end{array}\right)=\left(\begin{array}{c}
x \\
y \\
\dot{x} \\
\dot{y}
\end{array}\right),
$$

where the time index has been omitted. The angular velocity $\omega(\mathbf{x})$ of the target is modelled as the integrated effect on the target of each point along the boundary according to

$$
\omega(\mathbf{x})=d_{r}(\mathbf{x}) \int_{\mathbf{N}}\left(\beta_{d}+\beta_{a}\left(\dot{\mathbf{p}}_{\perp} \cdot \mathbf{l}(\mathbf{n})\right)\right) w(\mathbf{x}, \mathbf{n}) d \mathbf{n},
$$

where

- $\mathbf{N}$ denotes the set of all points along the boundary of the region;

- $d_{r}(\mathbf{x}) \in\{-1,1\}$ is the preferred rotational direction of the target;

- $\beta_{d}$ is a design parameter for the importance of avoiding collision with the boundary;

- $\beta_{a}$ is a design parameter for the importance of aligning with the boundary;

- $\mathbf{l}(\mathbf{n})$ is the tangent of the boundary in the point $\mathbf{n}$; and

- $w(\mathbf{x}, \mathbf{n})$ is the strength of the influence of a point $\mathbf{n}$ on a target with state $\mathbf{x}$.

The notation $(a, b)_{\perp}^{T}=(b,-a)^{T}$ is used, which rotates a two-dimensional vector $\pi / 2 \mathrm{rad}$ clockwise, and the operator $\mathbf{a} \cdot \mathbf{b}=\mathbf{a}^{T} \mathbf{b}$ is the scalar product of two vectors.

\subsubsection{Region Model}

The boundary of the constraint region is modelled as a simple two-dimensional polygon. The polygon is defined by $N$ vertices $\mathbf{v}_{i}, i \in\{1: N\}$, given in counterclockwise order. Points on each segment of the polygon are obtained from

$$
\mathbf{n}_{i}(s)=\mathbf{v}_{i}+s \mathbf{l}_{i}, \quad s \in\left[0, m_{i}\right],
$$

where $m_{i}=\left\|\mathbf{v}_{i+1}-\mathbf{v}_{i}\right\|$ and $\mathbf{l}_{i}=\left(\mathbf{v}_{i+1}-\mathbf{v}_{i}\right) / m_{i}$, as shown in Figure 2.1, with obvious adjustments for $m_{N}$ and $\mathbf{l}_{N}$.

The strength of the influence $w(\mathbf{x}, \mathbf{n})$ in (2.22) for a point $\mathbf{n}_{i}(s)$ on the boundary 


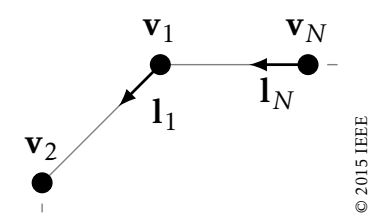

Figure 2.1: Polygon representation. Published in [112].

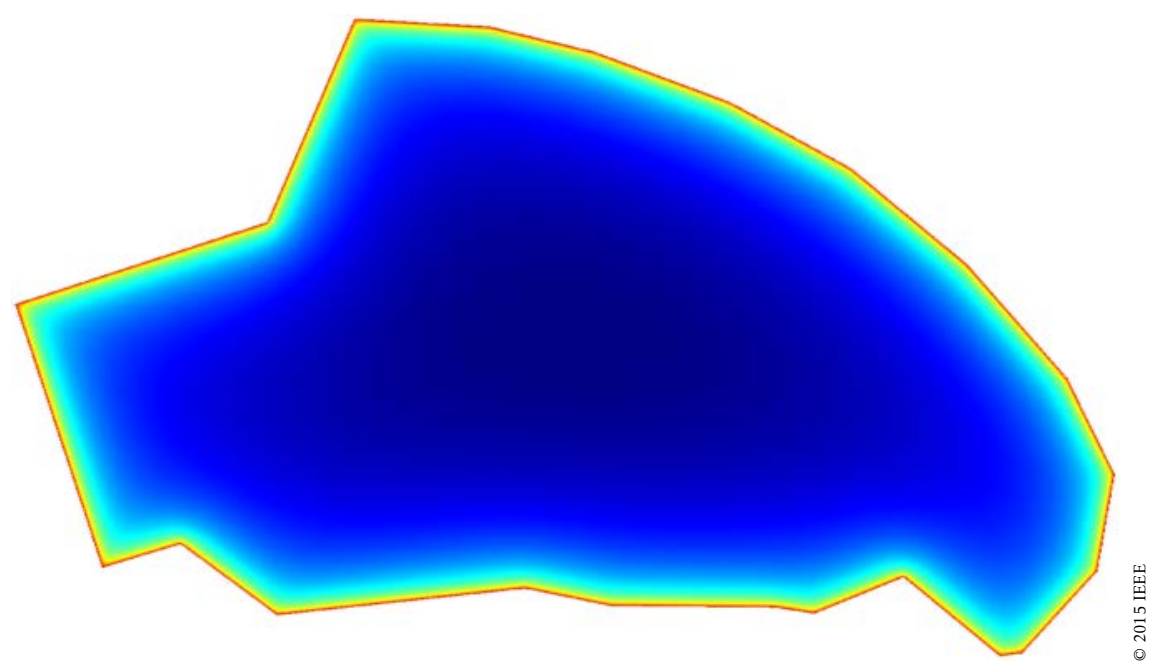

Figure 2.2: Potential field illustration. Published in [112].

is modelled to diminish as

$$
\tilde{w}_{i}(\mathbf{x}, s)=\frac{1}{\left\|\mathbf{p}-\mathbf{n}_{i}(s)\right\|^{2}}=\frac{1}{\left\|\mathbf{e}_{i}-s \mathbf{l}_{i}\right\|^{2}},
$$

where $\mathbf{e}_{i}=\mathbf{p}-\mathbf{v}_{i}$. Inserting (2.24) into (2.22) and using the region model in (2.23) gives the angular velocity

$$
\omega(\mathbf{x})=d_{r}(\mathbf{x}) \sum_{i=1}^{N}\left(\beta_{d}+\beta_{a}\left(\dot{\mathbf{p}}_{\perp} \cdot \mathbf{1}_{i}\right)\right) w_{i}(\mathbf{x}),
$$

where, using $\left\|\mathbf{1}_{i}\right\|=1$,

$$
\begin{aligned}
w_{i}(\mathbf{x}) & =\int_{0}^{m_{i}} \tilde{w}_{i}(\mathbf{x}, s) d s=\int_{0}^{m_{i}} \frac{1}{\left\|\mathbf{e}_{i}-s \mathbf{l}_{i}\right\|^{2}} d s \\
& =\frac{1}{\mathbf{l}_{i}^{T} \mathbf{e}_{i \perp}} \arctan \left[\frac{m_{i} \mathbf{l}_{i}^{T} \mathbf{e}_{i \perp}}{\left\|\mathbf{e}_{i}\right\|^{2}-m_{i} \mathbf{l}_{i}^{T} \mathbf{e}_{i}}\right] .
\end{aligned}
$$

Figure 2.2 illustrates (2.25) with $\beta_{a}=0$ for a constraint region. 
The Jacobians of the weights are given by

$$
\frac{\partial w_{i}}{\partial \mathbf{x}}(\mathbf{x})=\left(\begin{array}{llll}
\frac{\partial w_{i}}{\partial x}(\mathbf{x}) & \frac{\partial w_{i}}{\partial y}(\mathbf{x}) & 0 & 0
\end{array}\right)
$$

where, using the notation $\mathbf{a}=\left(a_{x}, a_{y}\right)^{T}$,

$$
\frac{\partial w_{i}}{\partial x}(\mathbf{x})=\frac{1}{\mathbf{1}_{i}^{T} \mathbf{e}_{i \perp}}\left(l_{i y} w_{i}(\mathbf{x})+\frac{e_{i y}}{\left\|\mathbf{e}_{i}\right\|^{2}}-\frac{e_{i y}-l_{i y} m_{i}}{\left\|\mathbf{e}_{i}\right\|^{2}-2 \mathbf{l}_{i}^{T} \mathbf{e}_{i} m_{i}+m_{i}^{2}}\right),
$$

and

$$
\frac{\partial w_{i}}{\partial y}(\mathbf{x})=\frac{1}{\mathbf{1}_{i}^{T} \mathbf{e}_{i \perp}}\left(-l_{i x} w_{i}(\mathbf{x})-\frac{e_{i x}}{\left\|\mathbf{a}_{i}\right\|^{2}}+\frac{e_{i x}-l_{i x} m_{i}}{\left\|\mathbf{e}_{i}\right\|^{2}-2 \mathbf{l}_{i}^{T} \mathbf{e}_{i} m_{i}+m_{i}^{2}}\right) .
$$

The direction of the rotation $d_{r}(\mathbf{x})$ can either be chosen using prior information or be estimated by comparing the target velocity $\dot{\mathbf{p}}$ to the boundary directions $\mathbf{l}_{i}$, e.g. using

$$
d_{r}(\mathbf{x})=\operatorname{sign}\left(\sum_{i=1}^{N}\left(\dot{\mathbf{p}} \cdot \mathbf{1}_{i}\right) w_{i}(\mathbf{x})\right) .
$$

Another option is to consider two models, one for each direction.

\subsubsection{Motion Model}

The motion model chosen is a coordinated turn model with known angular velocity [81]. The state vector is given by (2.21) and the deterministic motion model in continuous time is

$$
\dot{\mathbf{x}}(t)=\mathbf{F}_{c}(\omega(\mathbf{x}(t))) \mathbf{x}(t),
$$

where

$$
\mathbf{F}_{c}(\omega)=\left(\begin{array}{cccc}
0 & 0 & 1 & 0 \\
0 & 0 & 0 & 1 \\
0 & 0 & 0 & -\omega \\
0 & 0 & \omega & 0
\end{array}\right)
$$

With a zero-order hold assumption on $\omega(\mathbf{x}(t))=\omega$ in (2.30) and a discretisation time of $T,(2.30 \mathrm{~b})$ is discretised [99] exactly as

$$
\mathbf{F}(\omega)=\exp \left(\mathbf{F}_{c}(\omega) T\right)=\left(\begin{array}{cccc}
1 & 0 & \frac{\sin \omega T}{\omega} & -\frac{1-\cos \omega T}{\omega} \\
0 & 1 & \frac{1-\cos \omega T}{\omega} & \frac{\sin \omega T}{\omega} \\
0 & 0 & \cos \omega T & -\sin \omega T \\
0 & 0 & \sin \omega T & \cos \omega T
\end{array}\right) .
$$

Introducing additive white Gaussian noise results in the discrete-time motion model

$$
\mathbf{x}_{k}=\mathbf{f}\left(\mathbf{x}_{k-1}, \omega\left(\mathbf{x}_{k-1}\right)\right)+\mathbf{w}_{k}=\mathbf{F}\left(\omega\left(\mathbf{x}_{k-1}\right)\right) \mathbf{x}_{k-1}+\mathbf{w}_{k},
$$


where $\mathbf{f}(\mathbf{x}, \omega)=\mathbf{F}(\omega) \mathbf{x}, \mathbf{w}_{k} \sim \mathcal{N}\left(\mathbf{0}, \mathbf{Q}_{k}\right)$ and $\mathbf{Q}_{k}$ is defined as in (2.12).

Reintroducing $\omega=\omega(\mathbf{x})$, the Jacobian with regards to $\mathbf{x}$ is

$$
\frac{d \mathbf{f}}{d \mathbf{x}}(\mathbf{x}, \omega(\mathbf{x}))=\frac{\partial \mathbf{f}}{\partial \mathbf{x}}(\mathbf{x}, \omega(\mathbf{x}))+\frac{\partial \mathbf{f}}{\partial \omega}(\mathbf{x}, \omega(\mathbf{x})) \frac{\partial \omega(\mathbf{x})}{\partial \mathbf{x}},
$$

using the chain rule, where

$$
\begin{aligned}
\frac{\partial \mathbf{f}}{\partial \mathbf{x}}(\mathbf{x}, \omega) & =\left(\begin{array}{cccc}
1 & 0 & \frac{\sin \omega T}{\omega} & -\frac{1-\cos \omega T}{\omega} \\
0 & 1 & \frac{1-\cos \omega T}{\omega} & \frac{\sin \omega T}{\omega} \\
0 & 0 & \cos \omega T & -\sin \omega T \\
0 & 0 & \sin \omega T & \cos \omega T
\end{array}\right), \\
\frac{\partial \mathbf{f}}{\partial \omega}(\mathbf{x}, \omega) & =\left(\begin{array}{c}
\frac{(\omega T \dot{x}-\dot{y}) \cos (\omega T)-(\dot{x}+\omega T \dot{y}) \sin (\omega T)+\dot{y}}{\omega^{2}} \\
\frac{(\dot{x}-\omega T \dot{y}) \cos (\omega T)+(\dot{y}-\omega T \dot{x}) \sin (\omega T)-\dot{x}}{\omega^{2}} \\
-T \dot{y} \cos (\omega T)-T \dot{x} \sin (\omega T) \\
T \dot{x} \cos (\omega T)-T \dot{y} \sin (\omega T)
\end{array}\right),
\end{aligned}
$$

and, using (2.28),

$$
\frac{\partial \omega}{\partial \mathbf{x}}(\mathbf{x})=d_{r}(\mathbf{x}) \sum_{i=1}^{N}\left(\begin{array}{c}
\left(\beta_{d}+\beta_{a} \dot{\mathbf{p}}_{\perp}^{T} \mathbf{l}_{i}\right) \frac{\partial w^{i}}{\partial x}(\mathbf{x}) \\
\left(\beta_{d}+\beta_{a} \dot{\mathbf{p}}_{\perp}^{T} \mathbf{1}_{i}\right) \frac{\partial w^{i}}{\partial y}(\mathbf{x}) \\
-\beta_{a} l_{i y} w^{i}(\mathbf{x}) \\
\beta_{a} l_{i x} w^{i}(\mathbf{x})
\end{array}\right)^{T}
$$

Care is needed in implementations as $\omega \rightarrow 0$ for $(2.33 b)$ and $(2.33 c)$, where $(2.33 \mathrm{~b})$ reduces to

$$
\lim _{\omega \rightarrow 0} \frac{\partial \mathbf{f}}{\partial \mathbf{x}}(\mathbf{x}, \omega)=\left(\begin{array}{cccc}
1 & 0 & T & 0 \\
0 & 1 & 0 & T \\
0 & 0 & 1 & 0 \\
0 & 0 & 0 & 1
\end{array}\right),
$$

and $(2.33 c)$ reduces to

$$
\lim _{\omega \rightarrow 0} \frac{\partial \mathbf{f}}{\partial \omega}(\mathbf{x}, \omega)=\left(\begin{array}{llll}
-\frac{T^{2} \dot{y}}{2} & \frac{T^{2} \dot{x}}{2} & -T \dot{y} & T \dot{x}
\end{array}\right)^{T} .
$$

The motion model developed in this section is used in the dolphin application as described in Application 2.3. A simulation of the model is also presented.

\section{Application 2.3: Dolphin Motion Model}

The assumptions listed in Section 2.3.1 reasonably apply to dolphins swimming in a basin. Dolphins can swim in three dimensions, but the depth is less interesting in this application and difficult to measure, so only the two-dimensional movement is considered. Kolmården Wildlife Park has provided a map according to scale of the basin, and the boundary of the basin is modelled as a polygon by selecting points along the edge of the basin in the map such that a reasonable approximation of its shape is obtained, see Figure 2.3. 
Given that assumptions A2.1 hold, the model in (2.33) is used to model the motion of the dolphins. A simulation of a dolphin swimming according to this model is shown in Figure 2.3.

It should be noted that due to discretisation of the continuous model in (2.30), there is still a chance that the simulation of dolphins at high speeds might end up outside the basin. To mitigate this problem, a higher sampling rate can be used or a test can be applied after each simulation step to verify that the constraints still hold and, if not, heuristically move the dolphin back inside the basin. If the basin is non-convex, there might also be a problem of an invisible basin edge causing strange behaviour.

A comparison between the proposed CMM and the conventional NCVM and CTM in the dolphin application is presented in Application 2.4.

Application 2.4: Dolphin Model Comparison

Conventional models do not take the physical constraints into account, which is why the CMM is proposed. To show the differences in behaviour between the models, the prediction of each model with the resulting measurement uncertainty when no measurements are received is shown in Figure 2.4. The conventional models produce infeasible predictions and if the dolphin is rediscovered, due to a large gate or the prediction returning to the constraint region, the estimated trajectory is infeasible. The CMM prediction, however, follows the boundary of the constraint region with a covariance better adapted to the actual uncertainty of the position. The uncertainty in the velocities is propagated to the uncertainty in the position, causing rapidly increasing uncertainty in the position for the conventional models, while the CMM starts by increasing the uncertainty in position and then decreases it as the boundary is approached, although eccentricity still increases.

To improve the CMM further, the predictions from it could cover motions in both directions along a boundary until measurements have been acquired to distinguish which direction the dolphin chose.

\subsection{Sensor Models}

To enable inference in a motion model, observations of the state vectors are required. The sensor model describes the relationship between the state vector and the stochastic observation. A sensor model can be described by a conditional PDF, as in (2.1c). However, it is often difficult to accurately identify the probability density function for the sensor.

A convenient approximation is obtained by modelling the deterministic function $\mathbf{h}(\mathbf{x})$ of the state independently from the noise. The noise $\mathbf{v}_{j} \sim p_{\mathbf{v}_{j}}\left(\mathbf{v}_{j}\right)$ in the sensor can then be modelled to enter additively as

$$
\mathbf{y}_{j}=\mathbf{h}\left(\mathbf{x}_{j}\right)+\mathbf{v}_{j}
$$




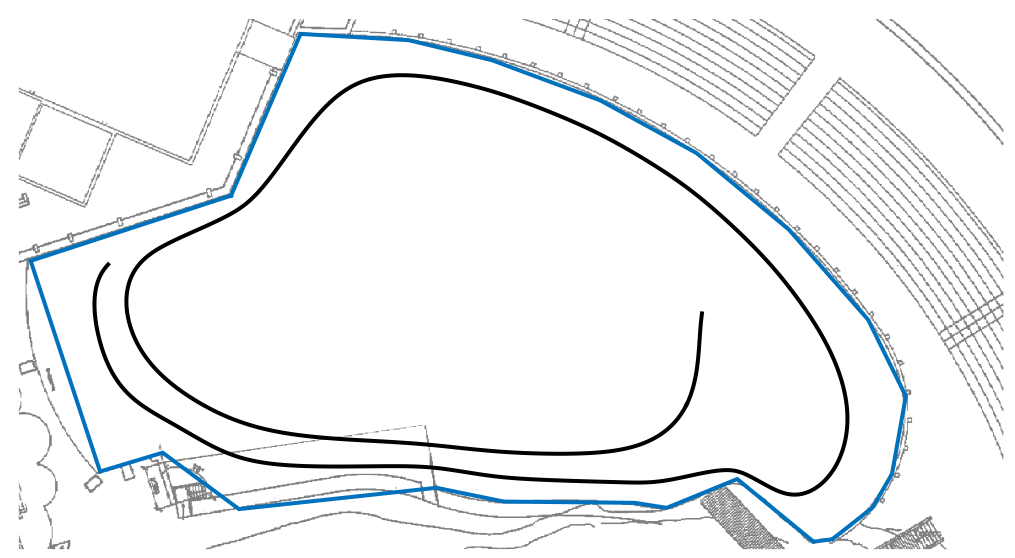

Figure 2.3: The basin edges are approximately described as a polygon, shown in blue. Straight segments of the edge have natural vertices for the polygon while curved segments require frequent vertices to achieve a reasonable approximation. Inside the basin is a simulation of a dolphin swimming according to the CMM in a clockwise manner.

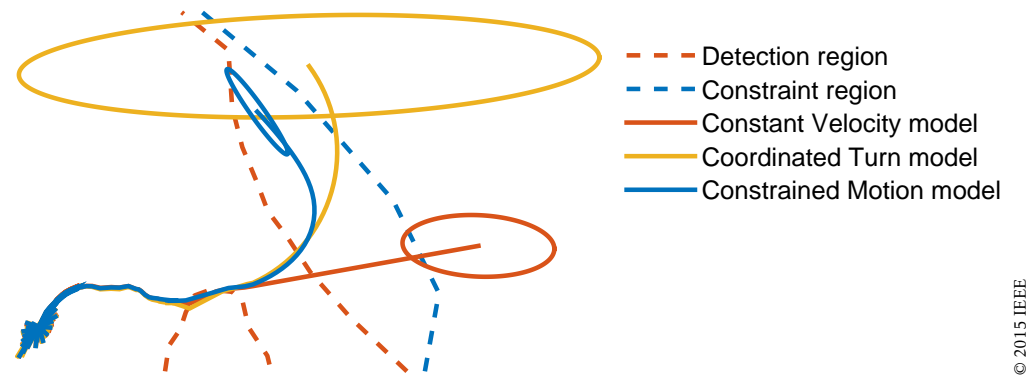

Figure 2.4: Compares the predictive capabilities and the measurement uncertainty of different models when a target stops generating measurements. The coordinated turn and constant velocity models do not take the constraint region into account resulting in infeasible predictions. The CMM keeps predictions within the constraint region. Published in [112]. 
For observation functions on the form $\breve{\mathbf{h}}(\mathbf{h}(\mathbf{x}))$, where $\breve{\mathbf{h}}(\mathbf{y})$ is bijective, the measurements $\breve{\mathbf{y}}_{j}$ can also be pre-processed before adding noise as

$$
\mathbf{y}_{j}=\breve{\mathbf{h}}^{-1}\left(\breve{\mathbf{y}}_{j}\right)=\mathbf{h}\left(\mathbf{x}_{j}\right)+\mathbf{v}_{j}
$$

If the measurement noise distribution is difficult to characterise, it is often convenient to approximate it with a zero-mean Gaussian with a reasonable covariance matrix $\mathbf{R}_{j}$. The matrix is chosen to compensate for measurement noise as well as model errors, and should rather be seen as the uncertainty of the measurement.

The function $\mathbf{h}(\mathbf{x})$, or at least its structure, can often be determined from the physical characteristics or geometry of the sensor. For many sensors it is common that the measurement pre-processing results in a direct observation of the position of the target, which is modelled as the linear function $\mathbf{h}(\mathbf{x})=(x, y)^{T}$. For other sensors the detection might be a range or an angle to the target. The range is modelled as $\mathbf{h}(\mathbf{x})=\sqrt{x^{2}+y^{2}}$ and the angle is modelled as $\mathbf{h}(\mathbf{x})=\arctan _{2}(y, x)$, which are nonlinear functions of the state. More advanced models for camera applications are described in Chapter 3.

Although a more advanced sensor model, described in Chapter 3, is used to pre-process the measurements in the dolphin application, a conventional sensor model is used in the SSM as described in Application 2.5.

Application 2.5: Dolphin Sensor Model

The dolphins are observed through a fisheye camera with a highly nonlinear sensor model. The model for the camera is discussed in Section 3.1 and since this model is bijective, it is possible to pre-process a measurement such that a twodimensional position of the dolphin in world coordinates is obtained. The measurement noise is then modelled to be Gaussian in world coordinates rather than in camera coordinates, resulting in the sensor model

$$
\mathbf{y}_{j}=\mathbf{H} \mathbf{x}_{j}+\mathbf{v}_{j}=\left(\begin{array}{llll}
1 & 0 & 0 & 0 \\
0 & 1 & 0 & 0
\end{array}\right) \mathbf{x}_{j}+\mathbf{v}_{j}
$$

where only the position of the dolphin is observed and $\mathbf{v}_{j} \sim \mathcal{N}(\mathbf{0}, \mathbf{R})$. The sensor model together with the CMM in Application 2.3 result in an AWGNSSM.

The structure of the sensor model used in the bird application is presented in Application 2.6, but the model itself is deferred to Chapter 3.

\section{Application 2.6: Bird Sensor Model}

The bird is observed through a camera, which is modelled as a perspective camera, and the sensor model used is on the form

$$
\mathbf{y}_{j}=\mathbf{h}\left(\mathbf{x}_{j}\right)+\mathbf{v}_{j},
$$

where the measurement function $\mathbf{h}\left(\mathbf{x}_{j}\right)$ is described in detail in Section 3.2 and $\mathbf{v}_{j} \sim \mathcal{N}(\mathbf{0}, \mathbf{R})$. The proposed sensor model together with the NCPM described in Application 2.1 result in an AWGNSSM. 
The sensor model in the orienteering application is linear, but requires an augmentation of the state vector as described in Application 2.7.

\section{Application 2.7: Orienteering Sensor Model}

The position of the sprinter is measured using a GNSS receiver. The GNSS is assumed to be biased by an offset due to occlusions, where the offset is modelled as a random walk. This results in an augmented state vector

$$
\mathbf{x}_{k}=\left(\begin{array}{llllll}
x_{k} & y_{k} & \dot{x}_{k} & \dot{y}_{k} & b_{k}^{x} & b_{k}^{y}
\end{array}\right)^{T} .
$$

The transition model for the offset $\mathbf{b}_{k}=\left(b_{k}^{x}, b_{k}^{y}\right)^{T}$ is

$$
\mathbf{b}_{k}=\alpha \mathbf{b}_{k-1}+\mathbf{w}_{k}^{b}, \quad \mathbf{w}_{k}^{b} \sim \mathcal{N}\left(\mathbf{0}, \mathbf{Q}^{b}\right),
$$

where the noise is independent and $\alpha \in[0,1]$ to make sure the offset returns to zero when no controls have been visited in a while. The sensor model for the GNSS measurements is

$$
\mathbf{y}_{k}=\mathbf{H}^{y} \mathbf{x}_{k}+\mathbf{v}_{k}^{y}, \quad \mathbf{v}_{k}^{y} \sim \mathcal{N}\left(\mathbf{0}, \mathbf{Q}^{y}\right),
$$

where

$$
\mathbf{H}^{y}=\left(\begin{array}{llllll}
1 & 0 & 0 & 0 & 1 & 0 \\
0 & 1 & 0 & 0 & 0 & 1
\end{array}\right),
$$

effectively selecting the sum of the position of the sprinter and the offset of the GNSS. The proposed sensor model together with the NCVM described in Application 2.2 result in a LGSSM.

\subsection{Jump Markov Model}

A jump Markov model (JMM) [36] is useful when a target exhibits a few distinct behaviours or the type of target is unknown. A common example is found in tracking of vehicles, which often move relatively straight and make distinct turns every once in a while. For such targets, the first behaviour is modelled well by an NCVM and the second by a CTM. In Section 2.5.1 the model is described in general and in Section 2.5.2 an extension to the model is proposed by the author, previously published in [23].

\subsubsection{General Model}

The JMM [36] considered is on the form

$$
\begin{aligned}
\mathbf{x}_{k} & =\mathbf{f}\left(\mathbf{x}_{k-1}, \delta_{k}\right)+\mathbf{w}_{k}, \\
\mathbf{y}_{j} & =\mathbf{h}_{j}\left(\mathbf{x}_{j}, \delta_{k}\right)+\mathbf{v}_{j}, \\
\mathbf{w}_{k} & \sim \mathcal{N}\left(\mathbf{0}, \mathbf{Q}_{k}\left(\delta_{k}\right)\right), \\
\mathbf{v}_{j} & \sim \mathcal{N}\left(\mathbf{0}, \mathbf{R}_{j}\left(\delta_{j}\right)\right), \\
p\left(\delta_{k} \mid \delta_{k-1}\right) & =\boldsymbol{\Pi}_{k}^{\delta_{k}, \delta_{k-1}},
\end{aligned}
$$


which is based on the AWGNSSM defined in (2.5)-(2.7). The AWGNSSM is extended with a discrete parameter $\delta_{k} \in \mathcal{S}$ representing the current mode of the system, where $\mathcal{S}$ is the set of modes. The prior probability at time step $k$ of transitioning from mode $a \in \mathcal{S}$ to mode $b \in \mathcal{S}$ is modelled by $\Pi_{k}^{b, a}$. The mode also exhibits the Markov property, resulting in a hidden Markov model for the mode. In some applications the main interest is the state, whereas in other applications the mode is more important.

The JMM is used to represent the different modes in the bird application as described in Application 2.8.

\section{Application 2.8: Bird Model}

The bird is considered to have two distinct behaviours, stationary and flight, where the first denotes the behaviour of the bird being still and scanning with its head near the centre of the funnel and the latter denotes the behaviour of the bird fluttering its wings trying to escape the funnel.

A JMM is used with the two modes stationary and flight, denoted $s$ and $f$ respectively. For the modes $\delta_{k} \in \mathcal{S}$, where $\mathcal{S}=\{s, f\}$, (2.43) is modelled to match the behaviour of the bird as closely as possible, based on the models presented in Applications 2.1 and 2.6.

As measurements, the position of the bird in the image is available as well as the amount of blurriness in the vicinity of the bird. The former measurement is utilised when modelling the modes as an indication of the amount of movement of the bird. In the stationary mode, small movements are expected, which is modelled as a small covariance $\mathbf{Q}(s)$, and in the flight mode, large movements are expected, which is modelled as a large covariance $\mathbf{Q}(f)$. The latter measurement is utilised when modelling the modes as an indication of the amount of wing flutter. The measurement of blur depends on the background light, which changes over time, so the level of blur is modelled as a random walk for each mode. Augmenting the state vector in Application 2.1 with the blur level for each mode results in

$$
\mathbf{x}_{k}=\left(\begin{array}{c}
x_{k} \\
y_{k} \\
b_{k}^{s} \\
b_{k}^{f}
\end{array}\right)=\left(\begin{array}{c}
\mathbf{p}_{k} \\
b_{k}^{s} \\
b_{k}^{f}
\end{array}\right),
$$

where $\mathbf{p}_{k}$ is the position of the bird and $b_{k}^{f}$ and $b_{k}^{s}$ are the blurriness levels for the two modes respectively. Using the measurement model in Application 2.6 the augmented SSM becomes

$$
\begin{array}{ll}
\mathbf{x}_{k}=\mathbf{x}_{k-1}+\mathbf{w}_{k}, & \mathbf{w}_{k} \sim \mathcal{N}\left(\mathbf{0}, \mathbf{Q}\left(\delta_{k}\right)\right), \\
\mathbf{y}_{k}=\left(\begin{array}{c}
\mathbf{h}\left(\mathbf{p}_{k}\right) \\
b_{k}^{\delta_{k}}
\end{array}\right)+\mathbf{v}_{k}, & \mathbf{v}_{k} \sim \mathcal{N}(\mathbf{0}, \mathbf{R}),
\end{array}
$$

with $\Pi^{\delta_{k}, \delta_{k-1}}$, covariances and prior distribution of the states tuned appropriately. 


\subsubsection{Model Extension}

In applications where the measurements are obtained through image processing, often an abundance of information can be extracted that might not directly relate to the state of the target, but might contain information directly dependent on the current mode. The model in (2.43) can then be extended with an additional measurement having the conditional distribution

$$
\mathbf{z}_{i} \sim p\left(\mathbf{z}_{i} \mid \delta_{i}\right), \quad i \in \mathcal{I} \subseteq \mathcal{K},
$$

where the conditional distribution is modelled to fit the application. Given the current mode $\delta_{k}$, the distribution is assumed to be conditionally independent from the states $\mathcal{X}_{1: k}$, the measurements $\mathcal{Y}_{1: k}$ and $\mathcal{Z}_{1: k-1}=\left\{\mathbf{z}_{i}\right\}_{i \in\{1: k-1\} \cap \mathcal{I}}$, and all previous modes $\Delta_{1: k-1}=\left\{\delta_{i}\right\}_{i=1}^{k-1}$ such that

$$
p\left(\mathbf{z}_{k} \mid \mathcal{Y}_{1: k}, \mathcal{Z}_{1: k-1}, \mathcal{X}_{1: k}, \Delta_{1: k}\right)=p\left(\mathbf{z}_{k} \mid \delta_{k}\right)
$$

The extension is used in the bird application as described in Application 2.9.

\section{Application 2.9: Bird Model Extension}

Since the funnel forces the bird back into the centre when it is stationary, the radial distance of the bird from the centre can be seen as an indication of the current mode. This is used as an additional measurement in the JMM in Application 2.8. In practice the radial distance is generated from the current state $\mathbf{x}_{k}$ as

$$
z_{k}=\sqrt{x_{k}^{2}+y_{k}^{2}}
$$

but will, with some abuse, be seen as a measurement from the image that is conditionally independent of the state given the current mode $\delta_{k}$. The distribution of $z_{k}$, given the current mode $\delta_{k}$, is modelled as

$$
p\left(z_{k} \mid \delta_{k}\right)= \begin{cases}c_{s} \cdot \mathcal{N}\left(z_{k} \mid 0, \sigma_{s}^{2}\right), & \text { if } 0 \leq z_{k} \leq r_{o} \text { and } \delta_{k}=s, \\ c_{f} \cdot \mathcal{N}\left(z_{k} \mid r_{o}, \sigma_{f}^{2}\right), & \text { if } 0 \leq z_{k} \leq r_{o} \text { and } \delta_{k}=f, \\ 0, & \text { otherwise, }\end{cases}
$$

where $r_{o}$ is the outer radius of the funnel, $c_{s}$ and $c_{f}$ are normalising constants and $\sigma_{s}^{2}$ and $\sigma_{f}^{2}$ are design parameters.

\subsection{Extended Target Model}

Traditionally, the resolution in sensors has been so low that each target has been assumed to generate at most one measurement in each time step [11]. However, for many modern sensors the assumption no longer holds since targets may occupy multiple resolution cells in the sensor [59]. Commonly, such targets are referred to as extended.

One option is to perform significant amounts of pre-processing to extract a single 
detection per target, resulting in loss of information which might be of interest. Another option is to explicitly model the extension of the target to maintain the information.

Many options exist for modelling extended targets $[17,55,60,116]$. In this thesis a model introduced in [79] is presented, where, in addition to the kinematic state vector, the extension is modelled as an ellipsoid represented by a random matrix. Although the exact shape of the target is not modelled this is sufficient for many applications [59].

Since a target can generate a number of measurements in each step, the set of measurements associated with the target is denoted

$$
\mathcal{Y}_{j}=\left\{\mathbf{y}_{j}^{i}\right\}_{i=1}^{N_{j}},
$$

where $N_{j}=\left|\mathcal{Y}_{j}\right|$ is the number of measurements associated with the target at time step $j$ and $\mathbf{y}_{j}^{i}$ is the $i$ th associated measurement at time step $j$. The measurements are assumed to be generated by independent and identically distributed processes. The SSM structures considered are a subset of LGSSMs including the motion models NCPM and NCVM. This is quite limiting, but still useful in many target tracking applications.

The measurement model is on the form

$$
p\left(\mathcal{Y}_{j} \mid N_{j}, \mathbf{x}_{j}, \mathbf{X}_{j}\right)=\prod_{i=1}^{N_{j}} \mathcal{N}\left(\mathbf{y}_{j}^{i} \mid\left(\tilde{\mathbf{H}}_{j} \otimes \mathbf{I}_{p}\right) \mathbf{x}_{j}, \mathbf{X}_{j}\right),
$$

where $\tilde{\mathbf{H}}_{j} \in \mathbb{R}^{1 \times s}$ is equal for all dimensions and $\mathbf{X}_{j} \in S_{++}^{p}$ is a symmetric positive definite random matrix that represents the extent of the target and $\mathbf{x}_{j} \in \mathbb{R}^{s p}$. The implication of this model is that the measurement noise is replaced by the extent of the target. The motivation is that the measurement noise for high resolution sensors is insignificant compared to the extent of the target [79].

The motion model for the target is on the form

$$
p\left(\mathbf{x}_{k} \mid \mathbf{X}_{k}, \mathbf{x}_{k-1}\right)=\mathcal{N}\left(\mathbf{x}_{k} \mid\left(\tilde{\mathbf{F}}_{k} \otimes \mathbf{I}_{p}\right) \mathbf{x}_{k-1}, \tilde{\mathbf{Q}}_{k} \otimes \mathbf{X}_{k}\right),
$$

where $\tilde{\mathbf{F}}_{k}$ is equal in all dimensions. The motivation for including the extent in the process noise covariance is that oblong targets often move along their length, increasing uncertainty along the major axis.

By viewing the extent as the covariance in a Gaussian distribution, a straightforward choice for the distribution of the extent is the inverse-Wishart distribution, which is a conjugate prior distribution for the covariance matrix of a Gaussian [53]. The initial extension is then modelled as

$$
p\left(\mathbf{X}_{0}\right)=\mathcal{I} \mathcal{W}\left(\mathbf{X}_{0} \mid v_{0}, \mathbf{V}_{0}\right) \propto\left|\mathbf{X}_{0}\right|^{v_{0} / 2} \exp \left(-\frac{1}{2} \operatorname{tr}\left(\mathbf{V}_{0} \mathbf{X}_{0}^{-1}\right)\right)
$$

where $\mathbf{X}_{0} \in S_{++}^{p}$ and $\mathbf{V}_{0} \in S_{++}^{p}$ are symmetric positive definite matrices. The 
model for the prediction is chosen heuristically as

$$
p\left(\mathbf{X}_{k} \mid \mathbf{X}_{k-1}\right)=\mathcal{I} \mathcal{W}\left(\mathbf{X}_{k} \mid e^{-T_{k} / \tau} v_{k-1}, \frac{e^{-T_{k} / \tau} v_{k-1}-2 p-2}{v_{k-1}-2 p-2} \mathbf{V}_{k-1}\right),
$$

where the degrees of freedom decay with a time constant $\tau$, symbolising increased uncertainty, and the expected value of the extent distribution,

$$
\mathbf{E}\left(\mathbf{X}_{k} \mid \mathbf{X}_{k-1}\right)=\frac{\mathbf{V}_{k}}{v_{k}-2 p-2}
$$

remains unaltered.

The extension model from this section is used with the NCVM in the savannah application as described in Application 2.10.

\section{Application 2.10: Savannah Animal Model}

The animals are tracked in image coordinates, so two dimensions for the positions are considered, resulting in a dimension $p$ of two. The quadcopter moves the camera in a relatively smooth manner, so an NCVM is a decent approximation of the animal movements, resulting in the model

$$
\tilde{\mathbf{F}}_{k}=\left(\begin{array}{ll}
1 & T \\
0 & 1
\end{array}\right),
$$

for each dimension, where $T$ is the inverse of the camera frame rate.

Since tracking is only performed in image coordinates, the measurement model for each dimension is simply

$$
\tilde{\mathbf{H}}_{j}=\left(\begin{array}{ll}
1 & 0
\end{array}\right) \text {. }
$$

The measurements $\mathcal{Y}_{j}$ are the pixels associated with a target in the image.

\subsection{Uncertain Timestamp Model}

Traditionally the timestamp of a measurement in an SSM is considered known and accurate. However, for some applications, this might not always be the case. The uncertain timestamp model (UTM) was developed by the author in [113] to model such scenarios and is restated in this section.

Consider the LGSSM in (2.8),

$$
\begin{array}{rlrl}
\mathbf{x}_{k} & =\mathbf{F}_{k} \mathbf{x}_{k-1}+\mathbf{w}_{k}, & \mathbf{w}_{k} \sim \mathcal{N}\left(0, \mathbf{Q}_{k}\right), \\
\mathbf{y}_{j}=\mathbf{H}_{j}^{y} \mathbf{x}_{j}+\mathbf{v}_{j}^{y}, & \mathbf{v}_{j}^{y} \sim \mathcal{N}\left(0, \mathbf{R}_{j}^{y}\right),
\end{array}
$$

with prior distribution $p\left(\mathbf{x}_{0}\right)=\mathcal{N}\left(\mathbf{x}_{0} \mid \overline{\mathbf{x}}_{0}, \mathbf{P}_{0}\right)$ on the state. In addition to the standard measurements, another observation,

$$
\mathbf{z}=\mathbf{H}^{z} \mathbf{x}_{\tau}+\mathbf{v}^{z}, \quad \mathbf{v}^{z} \sim \mathcal{N}\left(0, \mathbf{R}^{z}\right),
$$


is also available, where $\tau \sim p(\tau)$. The measurements $\mathbf{y}_{j} \in \mathbb{R}^{m^{y}}$ have precise timestamps and the observation $\mathbf{z} \in \mathbb{R}^{m^{z}}$ has an uncertain timestamp, limited to the interval $\tau \in \mathcal{K}$.

To simplify notation, $\mathcal{X} \triangleq\left\{\mathbf{x}_{k}\right\}_{k \in \mathcal{K}}$ and $\mathcal{Y} \triangleq\left\{\mathbf{y}_{j}\right\}_{j \in \mathcal{J}}$ will be used to denote the sets of all states and measurements, respectively, where both will be treated as column vectors where applicable.

A one-dimensional version of the UTM is used for analysing the impact of the additional observation in a simple uncertain time scenario as described in Application 2.11 .

\section{—_Application 2.11: Simple Uncertain Timestamp Model}

The simple scenario in Application 4.2 is modelled as a UTM. In principle the problem can be modelled in continuous time, however, as the involved integrals are difficult to compute even in the simplest of cases, $\tilde{\tau}$ is gridded, effectively substituting the involved integrals with sums. This results in a time-discrete SSM, with discretisation time $T$, where the time ensures sufficient temporal resolution.

The model for the simple scenario, after discretisation with $T=1 / N$ and $\tilde{\tau}=T \tau$, is defined by

$$
x_{k}=x_{k-1}+w_{k}, \quad w_{k} \sim \mathcal{N}(0, Q), \quad k \in \mathcal{K}=\{1: N\},
$$

and

$$
y_{j}=x_{j}+v_{j}^{y}, \quad v_{j}^{y} \sim \mathcal{N}\left(0, R^{y}\right) \quad j \in \mathcal{J}=\{1, N\},
$$

where the two standard measurements are $y_{1}$ and $y_{N}$. The observation is modelled as

$$
z=x_{\tau}+v^{z}, \quad v^{z} \sim \mathcal{N}\left(0, R^{z}\right),
$$

sampled at an uncertain time $\tau \in \mathcal{K}$, where the prior on $\tau$ is discretised as

$$
p(\tau) \propto p_{\tilde{\tau}}(T \tau)
$$

The resolution is chosen as $N=600$, the noise covariances as $Q=1.67 / N, R^{y}=$ 0.01 and $R^{z}=0.01$, the position prior distribution as nearly flat using $\bar{x}_{0}=0$ and $P_{0}=10^{9}$, and the measurements as $y_{1}=0$ and $y_{N}=1$. Two different cases are considered with the following parameters:

1. $z=0.5$ and $p(\tilde{\tau}) \propto 1$.

2. $z=1.5$ and $p(\tilde{\tau})=\mathcal{N}(\tilde{\tau} \mid 0.5,0.01)$.

The prior distributions $p(\tilde{\tau})$ are discretised using (2.60).

A two-dimensional version of this model is used in the orienteering application and the model is described in Application 2.12. 


\section{Application 2.12: Orienteering Uncertain Timestamp Model}

The motion model and sensor model for the GNSS are given in Applications 2.2 and 2.7, respectively. The observation, corresponding to the location of the control, is modelled as

$$
\mathbf{z}=\mathbf{H}^{z} \mathbf{x}_{\tau}+\mathbf{v}^{z}, \quad \mathbf{v}^{z} \sim \mathcal{N}\left(\mathbf{0}, \mathbf{R}^{z}\right),
$$

where

$$
\mathbf{H}^{z}=\left(\begin{array}{llllll}
1 & 0 & 0 & 0 & 0 & 0 \\
0 & 1 & 0 & 0 & 0 & 0
\end{array}\right),
$$

effectively selecting the position of the sprinter. 


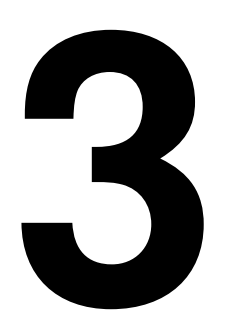

\section{Camera Sensor}

Cameras are versatile sensors with applications in a wide range of areas. In this thesis mainly stationary sensors are considered. They have fixed position, fixed angle and fixed field of view relative to the scene. The main advantage is that each pixel then corresponds to a particular position in the scenery, allowing for relatively simple detection of movement. Camera modelling is important in computer vision and more details on the subject are provided in, e.g., [84] and [66].

Common components of a camera model are described in Section 3.1. In Section 3.2 models for stationary cameras are considered and estimation is discussed for the models. In Section 3.3 a common method for background estimation using stationary cameras is presented. A slight modification is proposed, which was previously published by the author in [112], and a discussion is presented on rather heuristic approaches for foreground segmentation that can be applied for non-stationary cameras. A method for clustering and mapping pixels through nonlinear camera models is proposed in Section 3.4, previously presented by the author in [112].

\subsection{Camera Model}

There has been much research into computer vision in the last few decades, a field based on the camera as a versatile sensor. To infer knowledge related to world coordinates, a calibration of the camera is needed. Calibration is the estimation of a model relating world coordinates with pixels in the image recorded by the camera. Such models and their estimation are briefly described in this section. For a more thorough overview of computer vision and reconstruction from images see [84] and [66]. 
Given a number of world coordinates $\mathbf{x}_{i}^{r}=\left(x_{i}^{r}, y_{i}^{r}, z_{i}^{r}\right)^{T}$ and corresponding image coordinates $\mathbf{x}_{i}^{c}=\left(x_{i}^{c}, y_{i}^{c}\right)^{T}$ of a set of points, $i \in\{1: M\}$, one wants to find a map, or camera model, $\mathbf{x}^{c}=\mathbf{h}\left(\mathbf{x}^{r}\right)$ between the points. The map is in general nonlinear, but due to the geometry and physical properties of the camera a parametric model can be constructed to simplify the problem of calibrating the camera. A common method is to decompose the model into the components [84]

- camera extrinsics corresponding to the rotation and translation of the camera in world coordinates;

- a projection from three dimensions to two dimensions;

- camera intrinsics corresponding to field of view, principal point and pixel sizes;

- perspective compensation; and

- lens distortion.

One reason for this decomposition is that camera extrinsics might change dynamically while other components are camera dependent and do not change for a fixed focal length. To simplify notation homogenous coordinates are used, obtained by augmenting a coordinate vector by an element of one, denoted $\tilde{\mathbf{a}}=\left(\mathbf{a}^{T}, 1\right)^{T}$ for an arbitrary vector $\mathbf{a}$ in the sequel.

Camera extrinsics, projection, camera intrinsics and perspective compensation is given by

$$
\lambda \tilde{\mathbf{x}}^{u}=\mathbf{K} \Pi_{0} \mathbf{G} \tilde{\mathbf{x}}^{r},
$$

where $\mathbf{x}^{u}$ is the undistorted coordinate, $\lambda$ compensates for the perspective, ensuring homogenous coordinates and the projection is given by

$$
\Pi_{0}=\left(\begin{array}{llll}
1 & 0 & 0 & 0 \\
0 & 1 & 0 & 0 \\
0 & 0 & 1 & 0
\end{array}\right) .
$$

Camera intrinsics are modelled by

$$
\mathbf{K}=\left(\begin{array}{ccc}
\alpha_{x} & \gamma & p_{x} \\
0 & \alpha_{y} & p_{y} \\
0 & 0 & 1
\end{array}\right),
$$

where $\alpha_{x}=f \sigma_{x}$ and $\alpha_{y}=f \sigma_{y}$ model the focal length $f$ and pixel sizes $\sigma_{x}$ and $\sigma_{y}, \gamma$ models pixel skewness, and $\mathbf{p}=\left(p_{x}, p_{y}\right)^{T}$ is the principal point. See Figure 3.1 for an illustration of a projection onto the image plane for a scene already transformed into camera coordinates. Camera extrinsics are modelled by

$$
\mathbf{G}=\left(\begin{array}{cc}
\mathbf{R} & \mathbf{T} \\
\mathbf{0} & 1
\end{array}\right),
$$

where $\mathbf{R} \in S O(3)$ is a rotation matrix and $\mathbf{T} \in \mathbb{R}^{3}$ is a translation vector, mapping world coordinates into the coordinate system of the camera. 


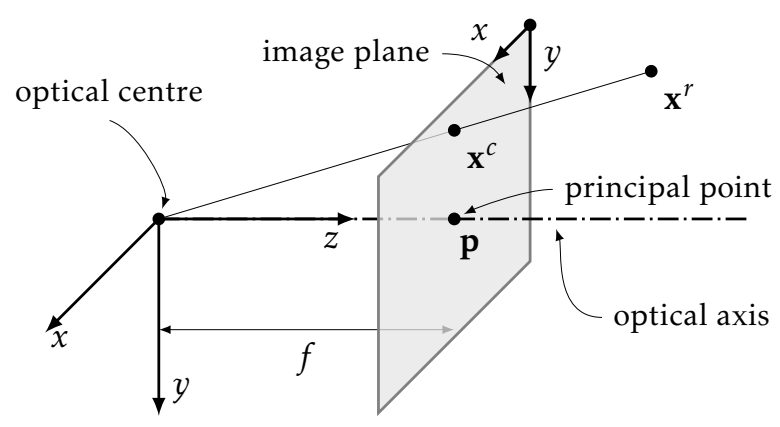

Figure 3.1: Illustrates the projection and the role of the focal length and principal point in camera calibration. The point $\mathbf{x}_{r}$ in the coordinate system of the camera is projected onto the image plane for a camera with focal length $f$ and principal point $\mathbf{p}$.

In case the lens distortion is minimal, a decent model is $\mathbf{x}^{c}=\mathbf{x}^{u}$, however, for wide angle lenses the distortion can be significant and is compensated for by a nonlinear transformation, $\mathbf{x}^{c}=\mathbf{f}\left(\mathbf{x}^{u}\right)$. The structure and parametrisation of the nonlinearity mainly depend on the type of distortion in the lens. A polynomial in the distance from the centre of distortion is suggested in [84] on the form

$$
\mathbf{x}^{c}=\mathbf{x}_{o}+\left(1+a_{1} r+a_{2} r^{2}+a_{3} r^{3}+\ldots\right)\left(\mathbf{x}^{u}-\mathbf{x}_{o}\right),
$$

where $r=\left\|\mathbf{x}^{u}-\mathbf{x}_{o}\right\|$ is the distance of the pixel from a centre of distortion $\mathbf{x}_{o} \in$ $\mathbb{R}^{2}$ that might be different from the principal point. Both the centre and the coefficients in the polynomial need to be estimated for the lens. This is a general model with many parameters, so for small $M$ a less flexible structure with fewer parameters might be preferred.

The process of estimating the parameters in $\mathbf{K}, \mathbf{G}$ and $\mathbf{f}(\mathbf{x})$ is called camera calibration and is often essential for tracking using cameras. Estimation of the parameters is quite involved and the algorithms are described in [123] and [84]. An implementation of the algorithms is available as open source [28]. A straightforward approach is to grab frames from the camera viewing a checker board pattern from different angles, making all the parameters, as well as the camera extrinsics, identifiable. In some cases this is not possible, and a tailored solution for the setup is required. One such approach is described in Section 3.2.

\subsection{Stationary Camera Modelling}

In some cases a complete calibration of the camera might not be possible, either because the camera is no longer available or it is mounted somewhere difficult to reach. Even if it were available, the mounted position of the camera in world coordinates might be difficult to estimate, reducing the usefulness of a traditional calibration. A different approach might therefore be more suitable in such cases. 
For a stationary camera, the camera extrinsics never change. The first three factors on the right hand side of (3.1) are therefore constant and the resulting matrix $\mathbf{H}=\mathbf{K} \Pi_{0} \mathbf{G}$ can be considered directly, reducing (3.1) to

$$
\lambda \tilde{\mathbf{x}}^{u}=\mathbf{H} \tilde{\mathbf{x}}^{r} .
$$

If an object in the video with distinguished features can be identified and has known dimensions in world coordinates, the model in (3.6) can be estimated directly using, e.g., a trust-region algorithm [32]. It is also possible to determine constraints on $\mathbf{H}$ in (3.6) for the estimation by deriving them from the camera model in (3.1).

In the bird application the dimensions of each funnel is known and the circles at the top and bottom are identified in the image. The circles are then used for camera calibration in Application 3.1.

\section{Application 3.1: Bird Camera}

The camera is mounted above four funnels placed in a square looking straight down as shown in Figure 1.2 and the vertical axis in the image is aligned with the magnetic field, with north towards the top of the image. The origin in world coordinates is placed at the centre of each funnel with north along the $y$-axis and the $z$-axis pointing out of the top of the funnel. This implies a rotation $\mathbf{R}=$ $\operatorname{diag}(1,-1,-1)$ of the camera, representing a half revolution around the world $x$ axis, and an unknown translation T. Furthermore, square pixels are assumed, reducing (3.3) to

$$
\mathbf{K}=\left(\begin{array}{ccc}
\alpha & 0 & p_{x} \\
0 & \alpha & p_{y} \\
0 & 0 & 1
\end{array}\right)
$$

and results in a parametrization $\mathbf{p}=\left(p_{1}, \ldots, p_{6}\right)^{T}$ of $\mathbf{H}$ as

$$
\mathbf{H}=\left(\begin{array}{cccc}
p_{1} & 0 & p_{2} & p_{3} \\
0 & -p_{1} & p_{4} & p_{5} \\
0 & 0 & -1 & p_{6}
\end{array}\right) .
$$

The lens distortion is assumed minimal and is modelled as $\mathbf{x}^{c}=\mathbf{x}^{u}$.

The dimensions of a funnel is given in world coordinates as the inner radius $r_{i}=125 \mathrm{~mm}$, the outer radius $r_{o}=325 \mathrm{~mm}$ and the height $h=155 \mathrm{~mm}$. Although some distortion occurs in the transformation, the inner and outer circles of the funnel are estimated approximately as circles directly from the video. By identifying coordinates on the form $\left(x^{c}, y^{c}\right)^{T}$ along the inner and outer circles a nonlinear least-squares estimate [93] is obtained for the parameters $\bar{x}, \bar{y}$ and $r$ of each circle represented on the form

$$
\left(x^{c}-\bar{x}\right)^{2}+\left(y^{c}-\bar{y}\right)^{2}=r^{2} .
$$

Matching coordinates to use for the calibration are then obtained by equiangularly sampling $M$ coordinates along the inner and outer circles in two dimensions for the image and in three dimensions for the funnel. The coordinates are 
represented as

$$
\begin{array}{ll}
\mathbf{x}_{i}^{c}=\left(\begin{array}{l}
x_{i}^{c} \\
y_{i}^{c}
\end{array}\right), & i \in\{1: M\}, \\
\mathbf{x}_{j}^{r}=\left(\begin{array}{l}
x_{i}^{r} \\
y_{i}^{r} \\
z_{i}^{r}
\end{array}\right), & i \in\{1: M\},
\end{array}
$$

where $z_{i}^{r}=-h / 2$ for the inner circle and $z_{i}^{r}=h / 2$ for the outer circle.

The perspective compensation makes the mapping defined in (3.6) nonlinear, so the trust-region algorithm described in [32] is used to solve for the unknown parameters $\mathbf{p}$ in (3.8). The model in (3.6) is used to derive the measurement function $\mathbf{h}(\mathbf{x})$ from world coordinates to image coordinates in (2.38).

The position of the bird along the $z$-axis in world coordinates cannot be reliably estimated using a single camera sensor, so the coordinate of the bird is assumed to be on the wall of the funnel at all times, which means the $z$-coordinate is computed as

$$
z^{r}=-\frac{h}{2}+h \cdot \min \left(\max \left(\sqrt{\left(x^{r}\right)^{2}+\left(y^{r}\right)^{2}}, r_{O}\right), r_{I}\right) /\left(r_{O}-r_{I}\right) .
$$

The resulting map for one of the funnels is shown in Figure 3.2.

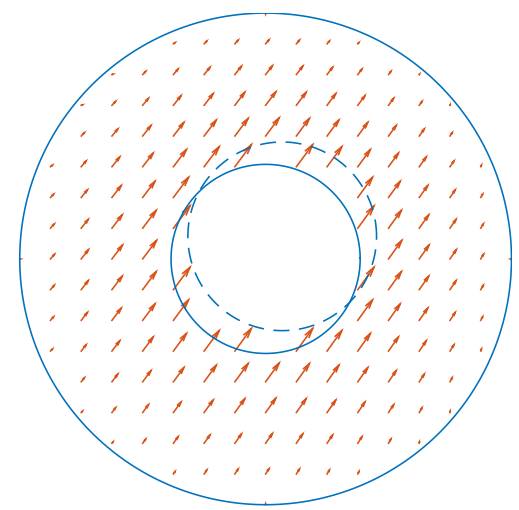

Figure 3.2: The funnel from above in world coordinates and scaled and translated image coordinates. The solid blue lines show the inner and outer circle in world coordinates and the dashed blue line shows the inner circle in image coordinates for comparison. The red arrows show the mapping for different parts of the funnel.

A blueprint of the basin in the dolphin application is available, see Figure 2.3, and points are manually matched between the blue print and the camera image, see Figure 1.1. The matched points are used to calibrate the camera in Application 3.2. 


\section{Application 3.2: Dolphin Camera with Fisheye Lens}

A camera with a fisheye lens, which exhibits severe radial distortion, is used for monitoring the dolphins as a solution to local regulations concerning audience integrity. It is mounted in the ceiling of the dolphinarium with most of the basin in its field of view, but not with a complete coverage. The blueprint is planar, so without loss of generality the surface of the basin can be placed at height 0 , reducing the problem to finding a map between two planes. With abuse of notation, the world coordinates $\mathbf{x}^{r}$ and $\mathbf{H}$ in (3.6) are for this example redefined as

$$
\begin{aligned}
\mathbf{x}^{r} & =\left(\begin{array}{l}
x^{r} \\
y^{r}
\end{array}\right), \\
\mathbf{H} & =\left(\begin{array}{lll}
H_{11} & H_{12} & H_{13} \\
H_{21} & H_{22} & H_{23} \\
H_{31} & H_{32} & H_{33}
\end{array}\right) .
\end{aligned}
$$

This simplification effectively assumes that everything seen in the camera exists at the surface of the water in the basin. Matrix $\mathbf{K}$ in (3.3) is always full rank considering $\alpha_{x} \neq 0$ and $\alpha_{y} \neq 0$, and matrix $\Pi_{0} \mathbf{G}$ given by (3.2) and (3.4) with the third column removed is full rank as long as the translation is out of the plane spanned by the $x$ - and $y$-axes in world coordinates, which is the case for the camera mounted in the ceiling. Thus, a properly estimated matrix $\mathbf{H}$ will be invertible. The mapping between undistorted image and world coordinates is therefore bijective, allowing also undistorted image coordinates to be mapped directly into world coordinates.

A distortion model also needs to be estimated for the fisheye lens. As discussed in Section 3.1, it is common to use a polynomial distortion model, but [41] suggests the following model for fisheye lenses

$$
\begin{aligned}
& r^{c}=R\left(r^{u}\right)=\frac{1}{\omega} \arctan \left(2 r^{u} \tan \frac{\omega}{2}\right), \\
& r^{u}=R^{-1}\left(r^{c}\right)=\frac{\tan \left(r^{c} \omega\right)}{2 \tan \frac{\omega}{2}},
\end{aligned}
$$

where $r^{c}=\left\|\mathbf{x}^{c}-\mathbf{x}_{o}\right\|$ is the radial distance from the centre of distortion $\mathbf{x}_{o}$ in the image, $r^{u}=\left\|\mathbf{x}^{u}-\mathbf{x}_{o}\right\|$ is the radial distance in the undistorted image, and $\omega$ is a parameter determining the amount of distortion. The mapping is bijective and computed as

$$
\begin{aligned}
& \mathbf{x}^{c}=\mathbf{x}_{o}+\frac{R\left(r^{u}\right)}{r^{u}}\left(\mathbf{x}^{u}-\mathbf{x}_{o}\right), \\
& \mathbf{x}^{u}=\mathbf{x}_{o}+\frac{R^{-1}\left(r^{c}\right)}{r^{c}}\left(\mathbf{x}^{c}-\mathbf{x}_{o}\right) .
\end{aligned}
$$

The method described in [124] is used to estimate $\mathbf{H}$ in (3.14) by finding the linear least-squares solution as an initial guess and refining it with a trust-region method [32]. The parameters $\omega$ and $\mathbf{x}_{o}$ are also estimated using the trust-region method. These solutions are computed in an alternated manner until convergence is achieved to find the complete mapping, as suggested by [124]. 


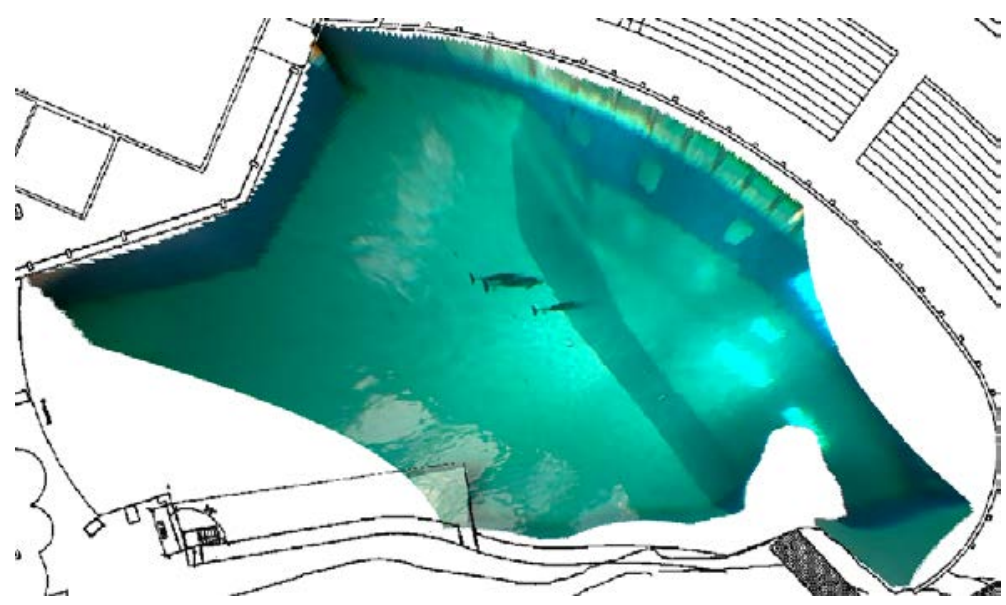

Figure 3.3: A video frame from the camera mounted in the dolphinarium. The image has been inverted using the estimated camera model and drawn into the map of the basin in world coordinates.

Having estimated the model parameters, (3.14) and (3.16b) is used to derive a measurement function from world coordinates to image coordinates. Further, since the mapping is static and bijective, the inverse function can also be used to map coordinates in the camera directly into world coordinates.

In Figure 3.3 a frame from the video has been inverted using the inverse mapping from image coordinates to world coordinates and drawn into the map of the basin. The mapping is not perfect in all parts of the image, but many issues caused by the fisheye lens have been mitigated.

\subsection{Foreground Segmentation}

To obtain detections of objects in the video it is segmented into background and foreground. For stationary cameras, a background model can be estimated over time for each pixel using, e.g., a mixture of Gaussians as in $[96,106]$ or quantiles as described in [31]. Estimation of a background model is difficult for nonstationary cameras. As an alternative, standard image processing methods, see e.g. [57], can be tailored to the particular application to estimate the background and to extract detections of the target.

For stationary cameras, a Gaussian-mixture background model $[96,106]$ is described in this section with some modifications proposed by the author. The basic idea is to estimate mixtures of Gaussians to represent the pixel intensities using the expectation-maximization (EM) algorithm [40]. Considering the large number of pixels, several approximations of this algorithm are applied to make computations less demanding. The intensities of a new image are gated and asso- 
ciated to the Gaussian components of the model. If an association is found, the model is updated, otherwise a new Gaussian is initialized with low weight. Gaussian components with large weights are considered background whereas those with small weights are considered to be foreground.

A single channel is considered for each pixel, resulting in one-dimensional Gaussian components. The Gaussian-mixture background model for each pixel consists of $K$ components with mean $\mu_{j}$, variance $\sigma_{j}^{2}$ and weight $w_{j}, j \in\{1: K\}$, sorted such that $w_{j} / \sigma_{j}$ is in descending order. The first $K_{B}$ components are considered background, where $K_{B}$ is determined as the smallest value such that $\sum_{j=1}^{K_{B}} w_{j}>T$, where $T \in[0,1]$ is a threshold. Note that most variables in this section implicitly depend on the time and the pixel, but that these dependences are not reflected explicitly unless necessary.

The parameter $\gamma^{2}$ determines the maximum squared Mahalanobis distance $d^{j}(I)$ considered a match through the criterion

$$
d^{j}(I)=\frac{\left(I-\mu_{j}\right)^{2}}{\sigma_{j}^{2}} \leq \gamma^{2},
$$

where $I$ is the currently measured intensity for the pixel. Selecting $\gamma^{2}$ is a tradeoff between tolerating variations in the background and detecting foreground. According to [106], it can be advantageous to replace $\gamma^{2}$ by $\gamma_{k}^{2}$, explicitly showing the dependence on time $t$, allowing the threshold to vary over time and different regions in the scene.

If no $j \leq K_{B}$ exists such that $d^{j}(I) \leq \gamma^{2}$ in (3.17), the pixel is considered to be part of the foreground. If no match is found at all, the last component is replaced by a new component with a mean at $I$ and default initial values for variance and weight. If a match is found for component $j$, its mean, variance and weight are updated as

$$
\begin{aligned}
P_{j} & =w_{j} \mathcal{N}\left(I \mid \mu_{j}, \Sigma_{j}\right), \\
w_{j}^{+} & =w_{j}+\alpha\left(P_{j}-w_{j}\right), \\
\rho_{j} & =\frac{\alpha P_{j}}{w_{j}^{+}}, \\
\mu_{j}^{+} & =\mu_{j}+\rho_{j}\left(I-\mu_{j}\right), \\
\Sigma_{j}^{+} & =\Sigma_{j}+\rho_{j}\left(\left(I-\mu_{j}\right)^{2}-\Sigma_{j}\right),
\end{aligned}
$$

where the superscript + denotes the updated values and $\alpha$ is a parameter that determines the learning speed. For all other components, the weights are reduced by a factor $1-\alpha$. The weights are then divided by the sum of all weights for renormalisation after each update.

A proposed extension to the method is to also compute

$$
d=\min _{j \leq K_{B}} d^{j}(I)
$$


for each foreground pixel, providing the Mahalanobis distance to the nearest background component. This value provides information on the degree of confidence for the detection, which could allow for more sophisticated methods in the post-processing to globally segment the foreground or improve the tracking as in [29], but is here used to weight individual measurements as described in Section 3.4. Additional extensions, such as using track predictions as feedback in the foreground segmentation, can be considered to improve the performance, e.g., as suggested by [108].

The foreground segmentation is generally noisy and is filtered using morphological operations [57], after which the output is $M_{f}$ observations consisting of the coordinates $\breve{\mathbf{y}}_{i}$ of the foreground pixels and their values $d_{i}$ obtained from (3.19). The set is denoted

$$
\breve{\mathcal{Y}}=\left\{\breve{\mathbf{y}}_{i}, d_{i}\right\}_{i=1}^{M_{f}}
$$

The method is used to extract detections of the dolphins in a challenging environment as described in Application 3.3.

\section{—Application 3.3: Dolphin Background Estimation}

The measurement pre-processing in the dolphin application aims at segmenting the foreground in the video. The camera is stationary, but the scene suffers from sunlight through large windows in the ceiling of the dolphinarium causing changes in the ambient light, reflections in the water and shadows at the bottom of the basin. To handle such dynamic light conditions, the method of estimating a Gaussian-mixture background model is applied as described in this section. The model is well suited for estimating the reflections in the background. This either results in several background components or an increased variance of a single component.

The method, as described, assumes an image with a single channel, which is obtained as a function of the red, green and blue channels. The function is chosen to achieve a reduction in the variance of the background pixels. Furthermore, the mean scene intensity is subtracted to make the model less sensitive to the light conditions.

To further improve robustness against changing light conditions, the threshold $\gamma_{k}^{2}$ is selected using the heuristics

$$
\gamma_{k}^{2}=\gamma_{0}^{2}+\gamma_{g}^{2} \max _{s \in[k-\tau, k]} \sqrt{\left|\bar{I}_{s}-\bar{I}_{s-1}\right|},
$$

where $\tau, \gamma_{0}^{2}$ and $\gamma_{g}^{2}$ are design parameters and $\bar{I}_{k}$ is the mean intensity in the image at time $k$. The second term increases the tolerance for all pixels when the light conditions in the scene change drastically for some time determined by $\tau$, allowing the current background components to adapt to the new conditions rather than to estimate new background components, which otherwise would result in many false detections.

To improve the performance, a detection region is defined in the image in the 


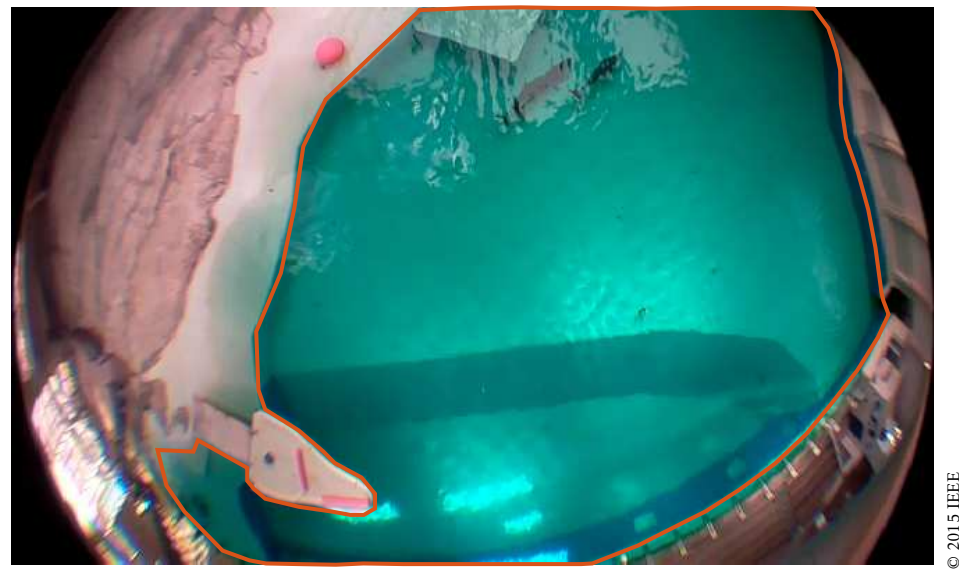

Figure 3.4: The detection region in the video, displayed as a red polygon, is the region of visible water in the basin where dolphins can be detected. Published in [112].

form of a polygon, masking out the visible part of the water in the basin where dolphins can possibly be detected. The detection region is shown in Figure 3.4. Since each pixel is modelled independently, the uneven shape of the region has only a limited effect on the result.

Each pixel is fed into the background estimation algorithm. Initially all pixels will be considered background by the algorithm, but after a number of iterations in a training period, the moving dolphins will be detected as foreground.

The extension described in (3.19) is used. It not only returns the estimated foreground, but also the statistical distance to the nearest background component. Some examples of segmented targets are shown in Figure 3.5. The quality of the output varies over the region and over time depending on, e.g., the stability of the background, separation of targets, camera resolution and distortion and light conditions. Although more information could be extracted using tailored computer vision techniques, thresholding together with morphological operations is sufficient for the intended target tracking and to use standard methods is beneficial when applying the same solution to similar problems.

A method modelling the background as moving quantiles is used for the bird application as outlined in Application 3.4.

Application 3.4: Bird Background Estimation

The background estimation in the bird application was provided by Dr. Kristoffer Öfjäll at Computer Vision Laboratory, Linköping University. The method used is presented in more detail in the appendix of [23]. A moving quantile model [31] is used for each pixel and an ellipse around the bird is estimated using channel decoding as described in [48]. This provides a measurement of the position and 


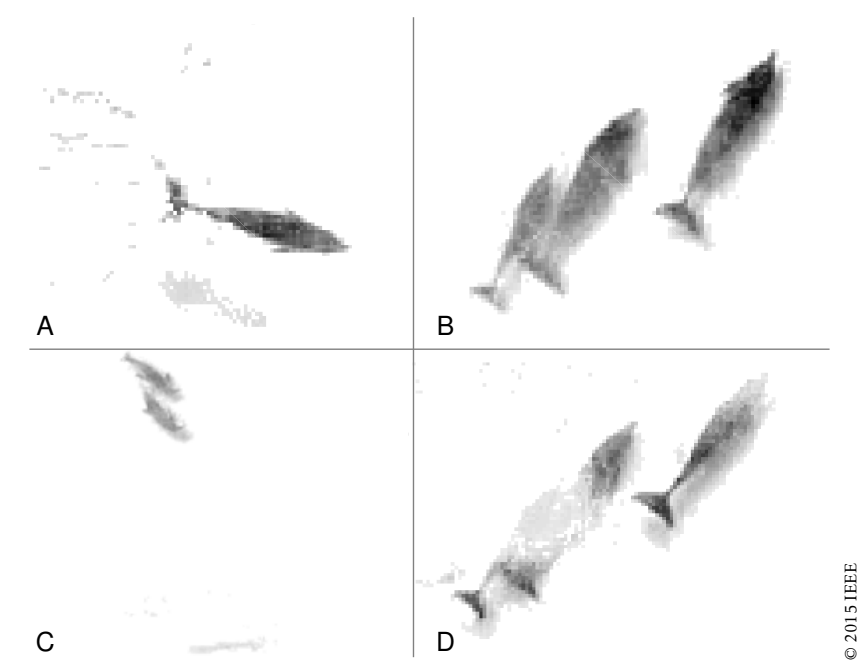

Figure 3.5: Shows the Mahalanobis distance output for segmented pixels in the foreground segmentation for four situations. A properly segmented target together with noise and a prominent shadow (A). Three separate dolphins where the two on the left would be combined by thresholding (B). A faint dolphin at the bottom $(C)$. Dolphins partly disappearing in a reflective region (D). Published in [112].

orientation of the bird. Wing flutter is then estimated as the amount of motion blur in foreground pixels. An example of a frame with the estimated background subtracted is shown in Figure 3.6.

For nonstationary cameras, estimating the background over time is a much more difficult task. With significant amounts of prior information, however, detections can instead be extracted using image processing methods tailored to the particular application. General image processing methods that can be applied are described in, e.g., [57]. Such a method is designed for the savannah application as presented in Application 3.5.

\section{Application 3.5: Savannah Animal Segmentation}

The camera in the Savannah application is non-stationary, so estimating the background over time is a difficult task. A simple heuristic method to estimate the background in a single frame is to apply the morphological operation open [57] with a structure larger than the expected size of the targets. The background is then subtracted from the original image, allowing for variations in the background over the scene. The estimated background is shown in Figure 3.7.

Computing the gradient [57] and adding to the image improves the contrast between the targets and the background, as is shown in Figure 3.8. A threshold is then applied to the image and the connected components [57] are extracted. All 


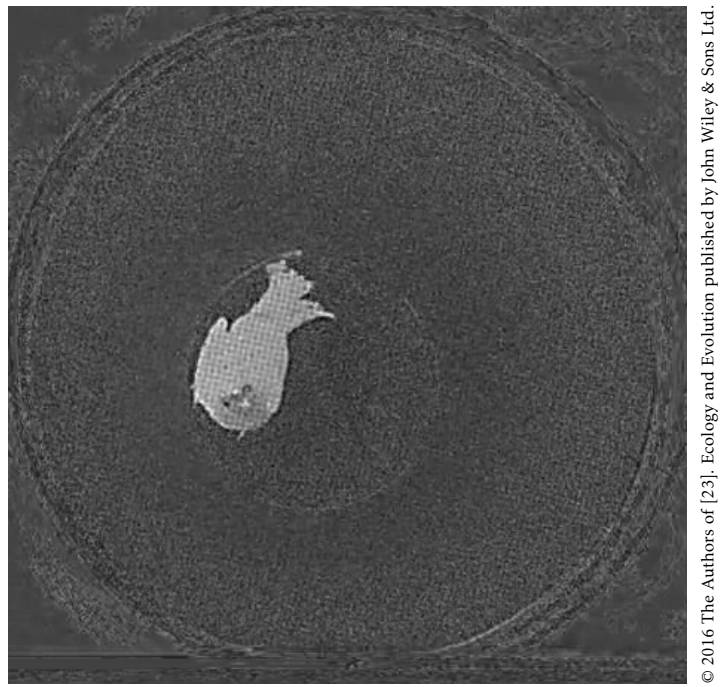

Figure 3.6: Example of a pre-processed image frame where the bird has been enhanced significantly by subtracting the estimated background. Published in [23].

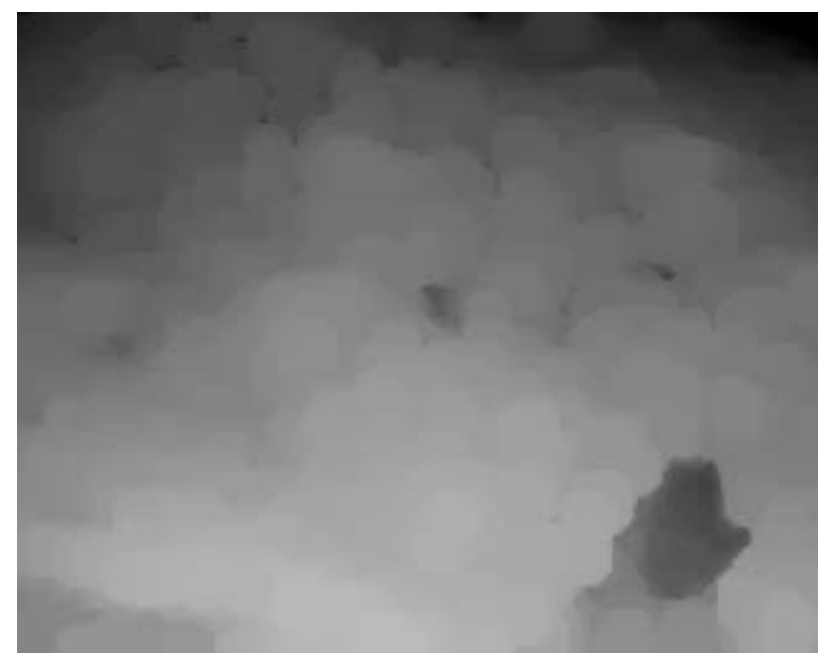

Figure 3.7: The estimation of the background using morphological operations for a single frame in the savannah application. 


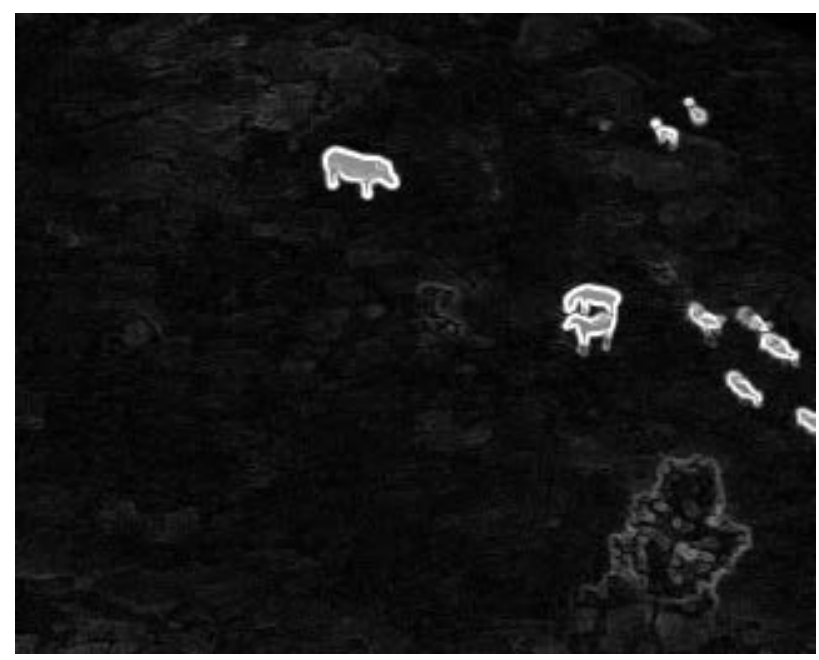

Figure 3.8: A single frame after background subtraction and gradient addition in the savannah application.

components with at least one pixel at a higher threshold are then extracted as detections, see Figure 3.9.

\subsection{Data Reduction}

There are multiple detections per target, which might be computationally expensive for a target tracking filter to process. In this section a method to reduce the amount of data is proposed by the author, although other solutions might be more suitable in some scenarios.

A first step is to obtain the indices $i$ of connected components $\tilde{\mathcal{C}}_{l}, l \in\{1: \tilde{M}\}$, from the measurements $\breve{\mathbf{y}}_{i}$ in (3.20) using the flood fill algorithm [57, Ch. 9].

The second step is to use the $k$-means clustering algorithm [85] on the measurements $\left\{\breve{\mathbf{y}}_{i}\right\}_{i \in \tilde{\mathcal{C}}_{l}}$ for each connected component to obtain the clusters $\mathcal{C}_{l}^{m}, m \in\{1$ : $\left.M_{l}\right\}$. To obtain clusters of approximate size $m_{r}$ the number of clusters for each component is chosen as $\left[\left|\tilde{\mathcal{C}}_{l}\right| / m_{r}\right]$, where $|\mathcal{A}|$ denotes the cardinality of set $\mathcal{A}$ and $\lceil a\rceil$ denotes the ceiling of $a$.

To reduce the number of measurements, the means are computed as

$$
\overline{\mathbf{y}}_{l}^{m}=\frac{1}{\left|\mathcal{C}_{l}^{m}\right|} \sum_{i \in \mathcal{C}_{l}^{m}} \breve{\mathbf{y}}_{i} \quad \text { and } \quad \bar{d}_{l}^{m}=\frac{1}{\left|\mathcal{C}_{l}^{m}\right|} \sum_{i \in \mathcal{C}_{l}^{m}} d_{i} .
$$

To keep some information regarding the extent of the connected component the 


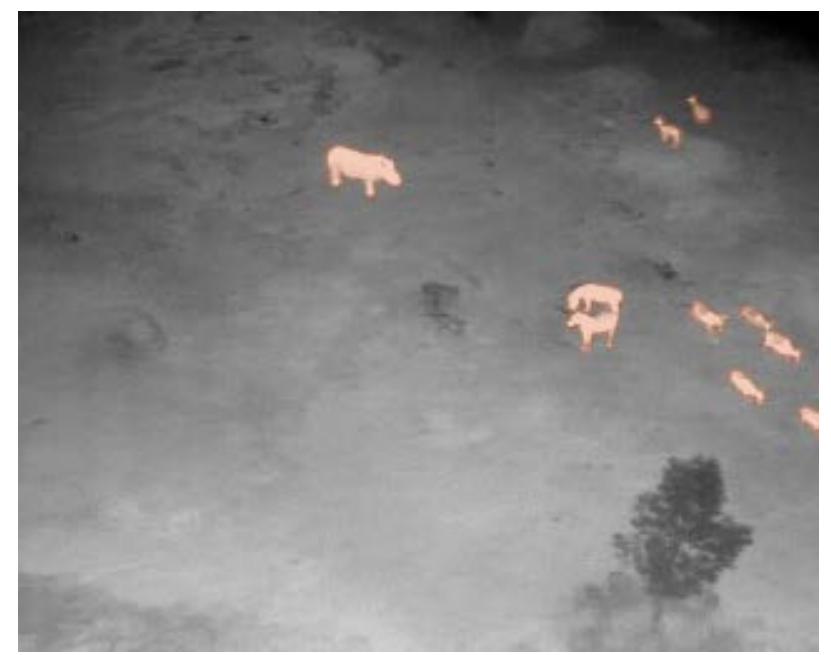

Figure 3.9: A single frame with detections marked in red in the savannah application.

covariance of the measurements is computed as

$$
\overline{\mathbf{Y}}_{l}^{m}=\frac{1}{\left|\mathcal{C}_{l}^{m}\right|} \sum_{i \in \mathcal{C}_{l}^{m}}\left(\breve{\mathbf{y}}_{i}-\overline{\mathbf{y}}_{l}^{m}\right)\left(\breve{\mathbf{y}}_{i}-\overline{\mathbf{y}}_{l}^{m}\right)^{T},
$$

and a reduced measurement set is obtained as

$$
\overline{\mathcal{Y}}=\bigcup_{l=1}^{\tilde{M}} \bigcup_{m=1}^{M_{l}}\left\{\overline{\mathbf{y}}_{l}^{m}, \bar{d}_{l}^{m}, \overline{\mathbf{Y}}_{l}^{m}\right\},
$$

with cardinality $M=|\overline{\mathcal{Y}}|$.

To exactly map the ellipsoids represented by the means and covariances in the reduced measurement set in (3.22b) using the nonlinear camera model in Section 3.2 is nontrivial. Since approximations have already been introduced, the extent is approximated using the unscented transform [73] of (3.22). The sigmapoints are mapped using a camera model and recomputed centroids and covariances are obtained. The measurements are also reindexed, resulting in the mapped reduced measurement set

$$
\mathcal{Y}=\left\{\mathbf{y}_{i}, d_{i}, \mathbf{Y}_{i}\right\}_{i=1}^{M} .
$$

The method is applied in Application 3.6 to the resulting set of measurements in Application 3.3. 


\section{Application 3.6: Dolphin Data Reduction}

An example of the segmented foreground for two dolphins, obtained as described in Application 3.3, is shown in Figure 3.10 together with the reduced measurements $\breve{\mathcal{Y}}$. The resulting mapped measurements $\mathcal{Y}$ are shown in Figure 3.11.

Figure 3.10: The segmented foreground is in grey scale, where a darker shade indicates a higher degree of confidence. On top are green ellipsoids representing the extents of the clusters, and a darker shade indicates a higher degree of confidence.

Figure 3.11: The extent of each cluster is mapped to world coordinates using an unscented transform and the camera model in Application 3.2. The shape has been distorted and scaled somewhat compared to Figure 3.10. 



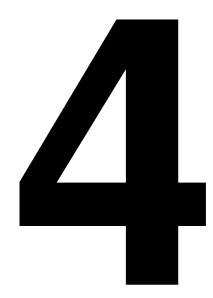

\section{State-Space Estimation}

Given a model and measurements as presented in Chapter 2, one wants to estimate the states and possibly other parameters in the model. This chapter presents theory on estimation for the models introduced in Chapter 2. Estimation for the LGSSM [74] and a common approximate estimation method for the NSSM [105] are described in more detail. There is an abundance of literature on the subject, for more details, see e.g. $[61,76,101]$.

The general Bayesian solution to the PSSM is discussed in Section 4.1. An exact closed-form solution for the filtering and smoothing distributions of the LGSSM are presented in Sections 4.2 and 4.3, respectively, as well as approximate methods for the NSSM. In Section 4.3.2 a method proposed by the author for updating the smoothed posterior in a single pass with an observation at an arbitrary time is derived. In Section 4.4 an approximate method for filtering in the JMM is described and in Section 4.4.2 filtering for the model extension proposed in Section 2.5.2 is outlined. The latter method was previously published by the author in [23]. Filtering for the extended target model in Section 2.6 is described in Section 4.5. The posterior distributions for the states and the timestamp in the UTM introduced in Section 2.7 are derived in Section 4.6 as well as some common point estimators, which was published by the author in [113].

\subsection{Bayesian Solution}

The Bayesian solutions for filtering and smoothing are outlined in this section. For more details, see e.g. [101].

To simplify expressions, the notations $\mathcal{X}_{a: b} \triangleq\left\{\mathbf{x}_{k}\right\}_{k=a}^{b}$ and $\mathcal{Y}_{a: b} \triangleq\left\{\mathbf{y}_{j}\right\}_{j \in\{a: b\} \cap \mathcal{J}}$ are defined with the shorthands $\mathcal{X} \triangleq \mathcal{X}_{0: N}$ and $\mathcal{Y} \triangleq \mathcal{Y}_{1: N}$. In some contexts $\mathcal{X}_{a: b}$ and 
$\mathcal{Y}_{a: b}$ will be treated as a column vector with the vectors in the elements stacked on top of each other.

The first case considered is the filtering distribution. This is the posterior distribution $p\left(\mathbf{x}_{k} \mid \mathcal{Y}_{1: k}\right)$ of state $\mathbf{x}_{k}$ conditional on all measurements $\mathcal{Y}_{1: k}$ up to and including time step $k$. This can be computed recursively using Bayes' theorem and the Chapman-Kolmogorov equations in a time update step

$$
\begin{aligned}
p\left(\mathbf{x}_{k} \mid \mathcal{Y}_{1: k-1}\right) & =\int p\left(\mathbf{x}_{k}, \mathbf{x}_{k-1} \mid \mathcal{Y}_{1: k-1}\right) d \mathbf{x}_{k-1} \\
& =\int p\left(\mathbf{x}_{k} \mid \mathbf{x}_{k-1}\right) p\left(\mathbf{x}_{k-1} \mid \mathcal{Y}_{1: k-1}\right) d \mathbf{x}_{k-1},
\end{aligned}
$$

where the integration is performed over the entire state space for $\mathbf{x}_{k-1}$, and a measurement update step, if a measurement is available,

$$
\begin{aligned}
p\left(\mathbf{x}_{k} \mid \mathcal{Y}_{1: k}\right) & =\frac{p\left(\mathbf{y}_{k} \mid \mathbf{x}_{k}, \mathcal{Y}_{1: k-1}\right) p\left(\mathbf{x}_{k} \mid \mathcal{Y}_{1: k-1}\right)}{p\left(\mathbf{y}_{k} \mid \mathcal{Y}_{1: k-1}\right)} \\
& \propto p\left(\mathbf{y}_{k} \mid \mathbf{x}_{k}\right) p\left(\mathbf{x}_{k} \mid \mathcal{Y}_{1: k-1}\right),
\end{aligned}
$$

where the Markov property is used in both steps. The symbol $\propto$ indicates equality up to a constant factor independent of $\mathbf{x}_{k}$ and the distribution can be recovered by normalisation. Given the filtering distribution $p\left(\mathbf{x}_{k-1} \mid \mathcal{Y}_{1: k-1}\right)$ of the state for the previous time step and a PSSM as defined in (2.1), all distributions are available, and for the initial state a prior distribution $p\left(\mathbf{x}_{0}\right)$ is assumed.

The second case considered is the smoothing distribution. This is the posterior distribution $p\left(\mathbf{x}_{k} \mid \mathcal{Y}\right)$ of state $\mathbf{x}_{k}$ conditional on all the measurements $\mathcal{Y}$. This distribution is slightly more complicated to compute because in addition to computing the filtering distributions, called the forward recursive equations, requires a recursion backwards, [78], given by

$$
p\left(\mathbf{x}_{k} \mid \mathcal{Y}\right)=p\left(\mathbf{x}_{k} \mid \mathcal{Y}_{1: k}\right) \int \frac{p\left(\mathbf{x}_{k+1} \mid \mathbf{x}_{k}\right) p\left(\mathbf{x}_{k+1} \mid \mathcal{Y}\right)}{p\left(\mathbf{x}_{k+1} \mid \mathcal{Y}_{1: k}\right)} d \mathbf{x}_{k+1} .
$$

Again, all distributions are available, either from the PSSM or the forward recursive equations.

In general these recursions cannot be computed in closed form, with the LGSSM defined in (2.8) being a notable exception, but require numerical methods.

\subsection{Kalman Filter}

As mentioned in Section 4.1, the filtering distribution for the LGSSM defined in (2.8) can be computed on closed form. The prior distribution of the initial state is Gaussian and it can be shown [101] that each step in the recursion results in a Gaussian distribution. The algorithm for computing the distribution is called the Kalman filter (KF) and was first derived in [74]. This section will present the $\mathrm{KF}$ for the LGSSM and some approximate solutions based on the KF for the NSSM. 
Given the posterior distribution $p\left(\mathbf{x}_{k-1} \mid \mathcal{Y}_{1: k-1}\right)=\mathcal{N}\left(\mathbf{x}_{k-1} \mid \hat{\mathbf{x}}_{k-1 \mid k-1}, \mathbf{P}_{k-1 \mid k-1}\right)$ for the state at time step $k-1$, the time update in (4.1) reduces to

$$
\begin{aligned}
p\left(\mathbf{x}_{k} \mid \mathcal{Y}_{1: k-1}\right) & =\int p\left(\mathbf{x}_{k} \mid \mathbf{x}_{k-1}\right) p\left(\mathbf{x}_{k-1} \mid \mathcal{Y}_{1: k-1}\right) d \mathbf{x}_{k-1} \\
& =\mathcal{N}\left(\mathbf{x}_{k} \mid \mathbf{F}_{k} \hat{\mathbf{x}}_{k-1 \mid k-1}, \mathbf{F}_{k} \mathbf{P}_{k-1 \mid k-1} \mathbf{F}_{k}^{T}+\mathbf{Q}_{k}\right) \\
& =\mathcal{N}\left(\mathbf{x}_{k} \mid \hat{\mathbf{x}}_{k \mid k-1}, \mathbf{P}_{k \mid k-1}\right),
\end{aligned}
$$

and the measurement update in (4.2) reduces to

$$
\begin{aligned}
p\left(\mathbf{x}_{k} \mid \mathcal{Y}_{1: k}\right) & =\frac{p\left(\mathbf{y}_{k} \mid \mathbf{x}_{k}, \mathcal{Y}_{1: k-1}\right) p\left(\mathbf{x}_{k} \mid \mathcal{Y}_{1: k-1}\right)}{p\left(\mathbf{y}_{k} \mid \mathcal{Y}_{1: k-1}\right)} \\
& =\mathcal{N}\left(\mathbf{x}_{k} \mid \hat{\mathbf{x}}_{k \mid k-1}+\mathbf{K}_{k}\left(\mathbf{y}_{k}-\mathbf{H}_{k} \hat{\mathbf{x}}_{k \mid k-1}\right), \mathbf{P}_{k \mid k-1}-\mathbf{K}_{k} \mathbf{H}_{k} \mathbf{P}_{k \mid k-1}\right) \\
& =\mathcal{N}\left(\mathbf{x}_{k} \mid \hat{\mathbf{x}}_{k \mid k}, \mathbf{P}_{k \mid k}\right),
\end{aligned}
$$

where

$$
\mathbf{K}_{k}=\mathbf{P}_{k \mid k-1} \mathbf{H}_{k}^{T}\left(\mathbf{H}_{k} \mathbf{P}_{k \mid k-1} \mathbf{H}_{k}^{T}+\mathbf{R}_{k}\right)^{-1} .
$$

See e.g. [101] for a derivation using joint and conditional distributions of Gaussians. Algorithm 4.1 conveniently summarises (4.4) and (4.5), where the mean and covariance of the posterior distribution are propagated over time. The measurement update step is omitted when no measurement is available at the current time step.

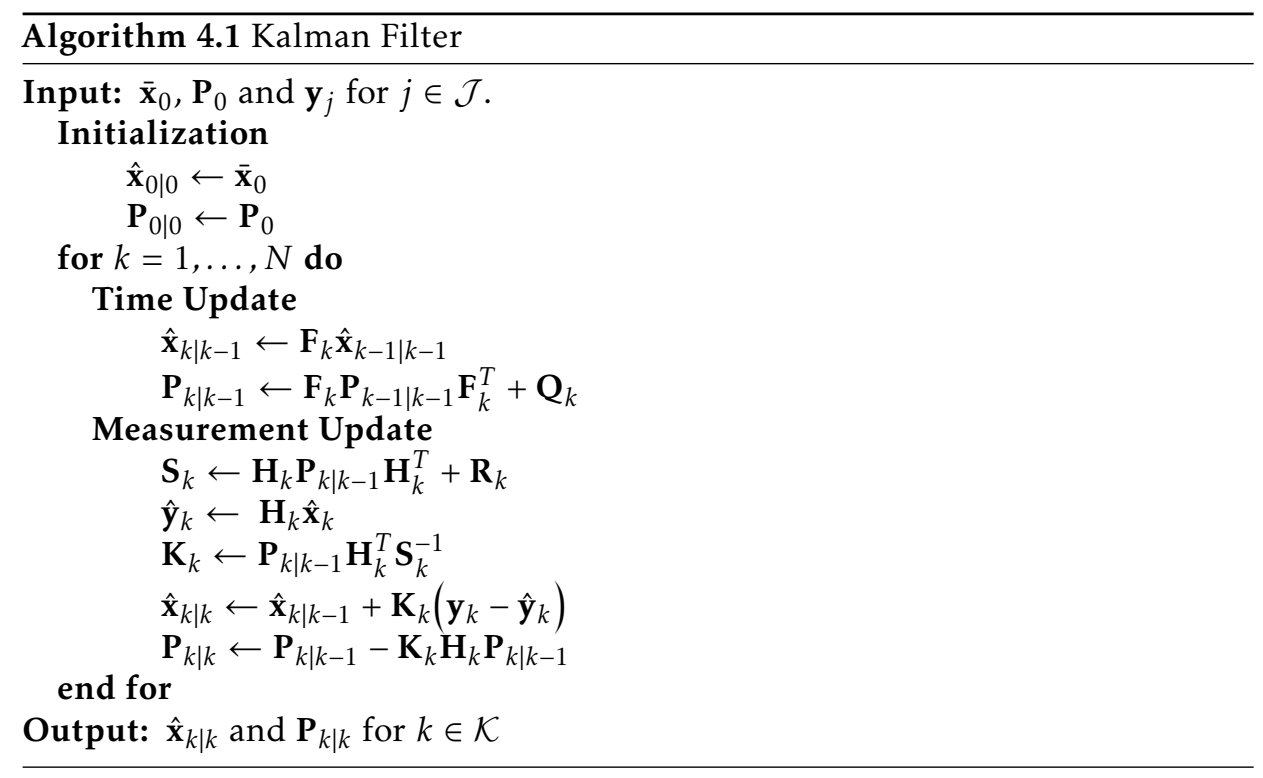

In the case of an NSSM, as defined in (2.3), the extended Kalman filter (EKF), first derived in [105], can be used to approximate the posterior distribution by its first two moments, the mean and the covariance. Affine approximations using the Jacobians of the transition and measurement functions and second moment approx- 
imations of the noise are used to propagate the mean and covariance over time. Algorithm 4.2 describes the steps involved in the EKF. The algorithm is no longer optimal and does not produce the exact Bayesian solution, but is common in practice due to its simplicity and decent performance in many applications [72]. However, there are many applications for which the algorithm does not perform well or even diverges, mainly where the linearisations or two-moment representations are poor approximations of the NSSM.

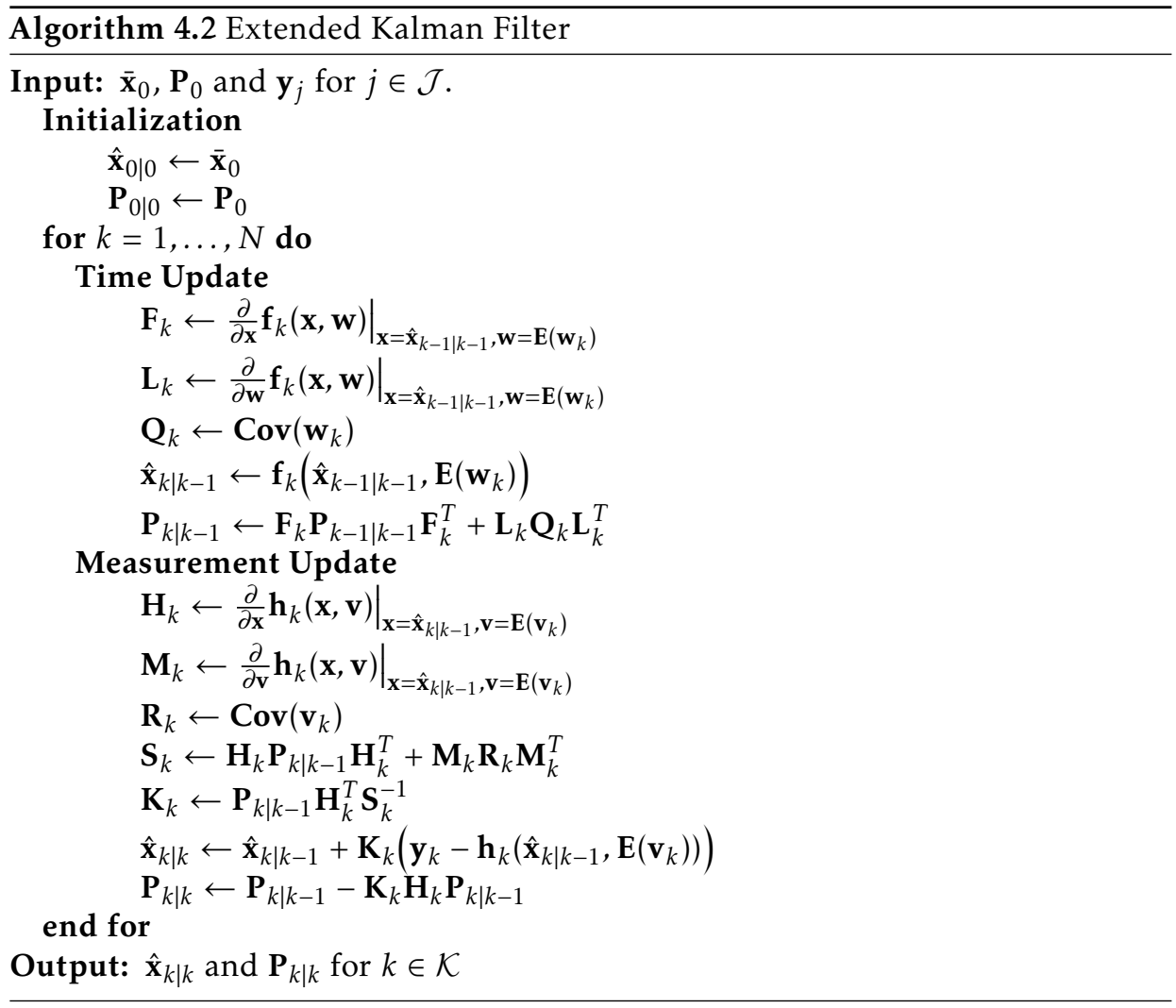

A common model in practice is the linear Gaussian motion model combined with a nonlinear measurement model with additive Gaussian noise. The iterated extended Kalman filter (IEKF), see [18], generally outperforms the EKF for this model at the expense of more computations. The basic idea is that the measurement update in the EKF can be seen as the first step in the Gauss-Newton method [93], and by applying the Gauss-Newton method until convergence the IEKF is obtained, resulting in a better approximation to the solution. The measurement update in Algorithm 4.2 is replaced by the measurement update in Algorithm 4.3. With $M=0$ the algorithm reduces to the EKF. The loop condition can also be replaced by a convergence criterion to improve performance.

The EKF is used for estimation in the dolphin application, however, considering 


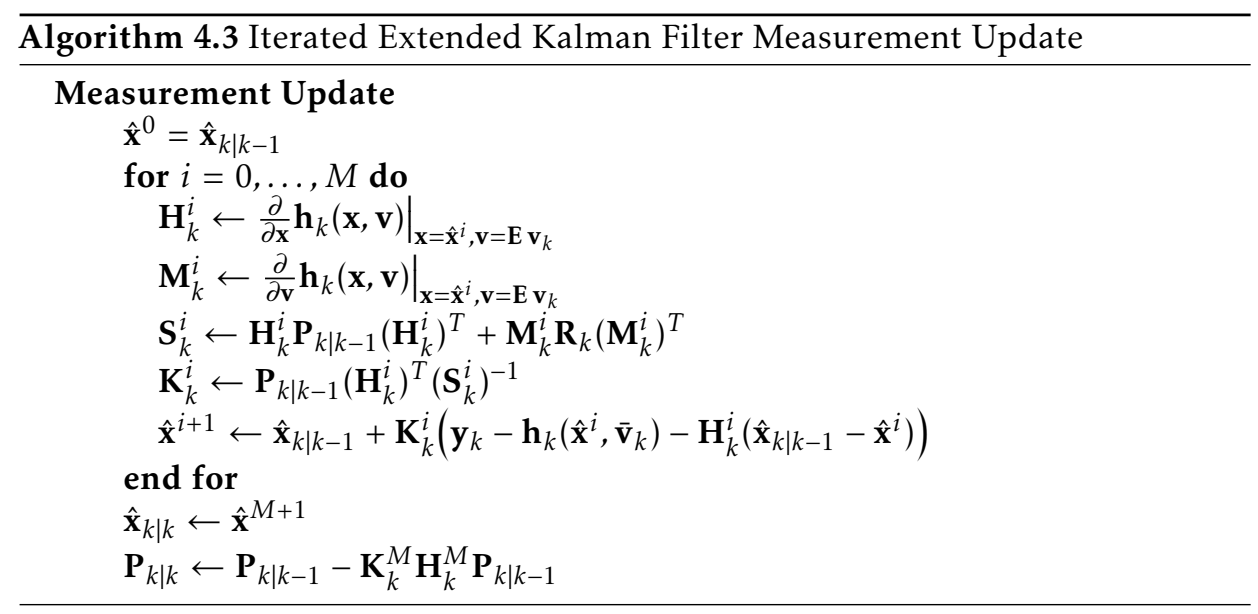

the association uncertainty in the application the presentation is deferred to Application 5.3 in Chapter 5. The IEKF is used in the filter bank for tracking in the bird application as described in Application 4.1 in Section 4.4.

\subsection{Linear Gaussian Smoother}

A method for computing the smoothing posterior distribution for the full state vector in the LGSSM is described [63] and a common two-pass recursive algorithm [101] is presented in Section 4.3.1. In Section 4.3.2 an algorithm is proposed by the author for updating the smoothing posterior distribution with an observation at an arbitrary timestamp in a single pass.

\subsubsection{General Smoothing}

When all measurements are available the smoothing distribution $p\left(\mathbf{x}_{k} \mid \mathcal{Y}\right)$ can be obtained as in (4.3). For an LGSSM, the posterior distribution is Gaussian and its parameters can be computed on closed form [30]. Any distribution $p\left(\mathbf{x}_{k} \mid \mathcal{Y}_{1: k+a}\right)$ for $a>0$ is commonly considered smoothing, however, only smoothing for an entire batch of measurements is considered in this section.

A straightforward solution is obtained by rearranging the LGSSM in (2.8) into a system of equations,

$$
\begin{aligned}
& \mathbf{0}=\mathbf{x}_{k}-\mathbf{F}_{k} \mathbf{x}_{k-1}+\mathbf{w}_{k}, \quad \mathbf{w}_{k} \sim \mathcal{N}\left(\mathbf{0}, \mathbf{Q}_{k}\right), \quad k \in \mathcal{K}, \\
& \mathbf{y}_{j}=\mathbf{H}_{j} \mathbf{x}_{j}+\mathbf{v}_{j}, \quad \mathbf{v}_{j} \sim \mathcal{N}\left(\mathbf{0}, \mathbf{R}_{j}\right), \quad j \in \mathcal{J}, \\
& \overline{\mathbf{x}}_{0}=\mathbf{x}_{0}+\mathbf{e}_{\mathbf{x}_{0}}, \quad \mathbf{e}_{\mathbf{x}_{0}} \sim \mathcal{N}\left(\mathbf{0}, \mathbf{P}_{0}\right),
\end{aligned}
$$

and rewriting on matrix form as

$$
\overline{\mathbf{Y}}=\overline{\mathbf{H}} \mathcal{X}+\mathbf{e}, \quad \mathbf{e} \sim \mathcal{N}(\mathbf{0}, \overline{\mathbf{R}}),
$$

where the matrices $\overline{\mathbf{Y}}, \overline{\mathbf{H}}$ and $\overline{\mathbf{R}}$ are chosen such that the system of equations 
in (4.7) is represented. For affine models, $\bar{Y}$ is modified to account for the constant term. The posterior distribution of the full state vector is then computed as [95]

$$
\begin{aligned}
& p(\mathcal{X} \mid \mathcal{Y}) \\
& \propto p(\mathcal{Y} \mid \mathcal{X}) p(\mathcal{X}) \\
& \propto \exp \left(-\frac{1}{2}\left(\left\|\mathbf{x}_{0}-\overline{\mathbf{x}}_{0}\right\|_{\mathbf{P}_{0}}^{2}+\sum_{k \in \mathcal{K}}\left\|\mathbf{x}_{k}-\mathbf{F}_{k} \mathbf{x}_{k-1}\right\|_{\mathbf{Q}_{k}}^{2}+\sum_{j \in \mathcal{J}}\left\|\mathbf{y}_{j}-\mathbf{H}_{j} \mathbf{x}_{j}\right\|_{\mathbf{R}_{j}}^{2}\right)\right) \\
& \propto \exp \left(-\frac{1}{2}(\overline{\mathbf{Y}}-\overline{\mathbf{H}} \mathcal{X})^{T} \overline{\mathbf{R}}^{-1}(\overline{\mathbf{Y}}-\overline{\mathbf{H}} \mathcal{X})\right) \\
& =\exp \left(-\frac{1}{2}\left(\overline{\mathbf{Y}}^{T} \overline{\mathbf{R}}^{-1} \overline{\mathbf{Y}}-2 \overline{\mathbf{Y}}^{T} \overline{\mathbf{R}}^{-1} \overline{\mathbf{H}} \mathcal{X}+\mathcal{X}^{T} \overline{\mathbf{H}}^{T} \overline{\mathbf{R}}^{-1} \overline{\mathbf{H}} \mathcal{X}\right)\right) \\
& \propto \exp \left(-\frac{1}{2}\left(\overline{\mathbf{Y}}^{T} \overline{\mathbf{R}}^{-1} \overline{\mathbf{H}}\left(\overline{\mathbf{H}}^{T} \overline{\mathbf{R}}^{-1} \overline{\mathbf{H}}\right)^{-1}\left(\overline{\mathbf{H}}^{T} \overline{\mathbf{R}}^{-1} \overline{\mathbf{H}}\right)\left(\overline{\mathbf{H}}^{T} \overline{\mathbf{R}}^{-1} \overline{\mathbf{H}}\right)^{-1} \overline{\mathbf{H}}^{T} \overline{\mathbf{R}}^{-1} \overline{\mathbf{Y}}-\right.\right. \\
& \left.\left.2 \overline{\mathbf{Y}}^{T} \overline{\mathbf{R}}^{-1} \overline{\mathbf{H}}\left(\overline{\mathbf{H}}^{T} \overline{\mathbf{R}}^{-1} \overline{\mathbf{H}}\right)^{-1}\left(\overline{\mathbf{H}}^{T} \overline{\mathbf{R}}^{-1} \overline{\mathbf{H}}\right) \mathcal{X}+\mathcal{X}^{T} \overline{\mathbf{H}}^{T} \overline{\mathbf{R}}^{-1} \overline{\mathbf{H}} \mathcal{X}\right)\right) \\
& =\exp \left(-\frac{1}{2}\left\|\left(\overline{\mathbf{H}}^{T} \overline{\mathbf{R}}^{-1} \overline{\mathbf{H}}\right)^{-1} \overline{\mathbf{H}}^{T} \overline{\mathbf{R}}^{-1} \overline{\mathbf{Y}}-\mathcal{X}\right\|_{\overline{\mathbf{H}}^{T} \overline{\mathbf{R}}^{-1} \overline{\mathbf{H}}}^{2}\right) \\
& \propto \mathcal{N}\left(\mathcal{X} \mid\left(\overline{\mathbf{H}}^{T} \overline{\mathbf{R}}^{-1} \overline{\mathbf{H}}\right)^{-1} \overline{\mathbf{H}}^{T} \overline{\mathbf{R}}^{-1} \overline{\mathbf{Y}},\left(\overline{\mathbf{H}}^{T} \overline{\mathbf{R}}^{-1} \overline{\mathbf{H}}\right)^{-1}\right) .
\end{aligned}
$$

A recursive solution to the posterior distribution of the marginal state vector can be obtained using the Rauch-Tung-Striebel smoother (RTSS) [97]. The RTSS is executed in a forward and a backward recursion, where the former is equivalent to the KF. The mean vectors $\hat{\mathbf{x}}_{k \mid k-1}$ and $\hat{\mathbf{x}}_{k \mid k}$ and covariances $\mathbf{P}_{k \mid k-1}$ and $\mathbf{P}_{k \mid k}, k \in \mathcal{K}$, in the prediction distributions (4.4) and the filtering distributions (4.5), respectively, are stored. The backward recursion in (4.3) is computed as

$$
\begin{aligned}
& \hat{\mathbf{x}}_{k-1 \mid N}=\hat{\mathbf{x}}_{k-1 \mid k-1}+\mathbf{P}_{k-1 \mid k-1} \mathbf{F}_{k}^{T} \mathbf{P}_{k \mid k-1}\left(\hat{\mathbf{x}}_{k \mid N}-\hat{\mathbf{x}}_{k \mid k-1}\right), \\
& \mathbf{P}_{k-1 \mid N}=\mathbf{P}_{k-1 \mid k-1}+\mathbf{P}_{k-1 \mid k-1} \mathbf{F}_{k}^{T} \mathbf{P}_{k \mid k-1}\left(\mathbf{P}_{k \mid N}-\mathbf{P}_{k-1 \mid k-1}\right) \mathbf{P}_{k \mid k-1} \mathbf{F}_{k} \mathbf{P}_{k-1 \mid k-1} .
\end{aligned}
$$

The RTSS is outlined in Algorithm 4.4, and it results in the marginalised posterior distribution given by

$$
p\left(\mathbf{x}_{k} \mid \mathcal{Y}\right)=\mathcal{N}\left(\mathbf{x}_{k} \mid \hat{\mathbf{x}}_{k \mid N}, \mathbf{P}_{k \mid N}\right)
$$

The RTSS can be also be applied to NSSMs by linearising the nonlinear functions as described in [18]. This method resembles the approach in the EKF for the filtering distribution. The forward recursion is replaced by an EKF in the first iteration. An affine version of the RTSS is then applied iteratively until convergence with a new linearisation between each iteration at the current estimate. As for the EKF, the posterior distribution is approximate and no convergence guarantees exist.

The RTSS is used for estimation in the simple uncertain time scenario and the orienteering application, however, considering the uncertainty in time the presentation is deferred to Section 4.6. 


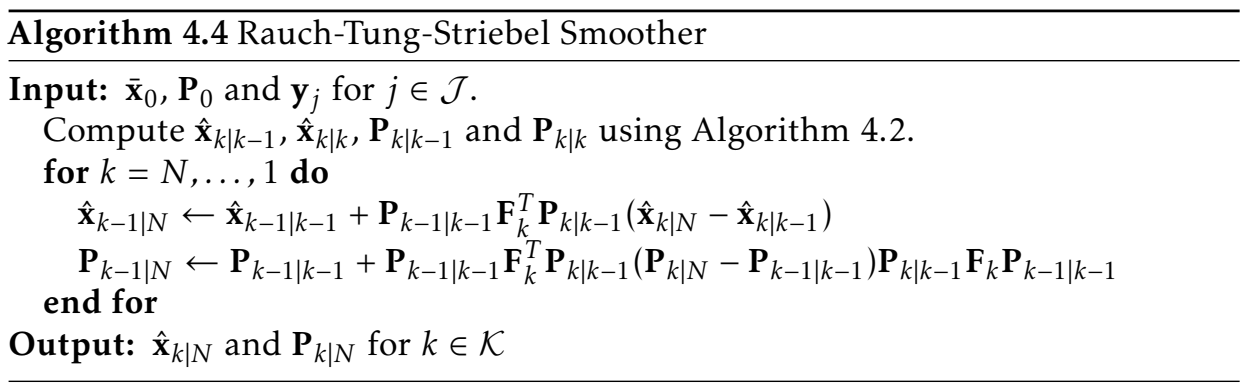

\subsubsection{Update of Posterior Distribution}

In some situations a smoothing posterior distribution is already computed and one is interested in adding an additional measurement at some arbitrary time. A straightforward approach is to recompute the smoothing distribution, but an alternative is derived in this section that only requires a single pass.

Given is a posterior distribution $p(\mathcal{X} \mid Y)$ of the full state vector $\mathcal{X}$ given the measurements $\mathcal{Y}$ and also an additional observation $\mathbf{z}$ at time $\tau$, modelled as $\mathbf{z}=\mathbf{H} \mathbf{x}_{\tau}+\mathbf{v}$, where $\mathbf{v} \sim \mathcal{N}(0, \mathbf{R})$. The joint distribution of the states and the new observation is given by [63]

$$
\begin{aligned}
p(\mathcal{X}, \mathbf{z} \mid \mathcal{Y}) & =\mathcal{N}\left(\left[\begin{array}{c}
\mathcal{X} \\
\mathbf{z}
\end{array}\right] \mid\left[\begin{array}{c}
\hat{\mathcal{X}} \\
\overline{\mathbf{H}}_{\tau} \hat{\mathcal{X}}
\end{array}\right],\left[\begin{array}{cc}
\mathbf{P} & \mathbf{P}^{\mathbf{H}_{\tau}^{T}} \\
\overline{\mathbf{H}}_{\tau} \mathbf{P} & \overline{\mathbf{H}}_{\tau} \mathbf{P} \overline{\mathbf{H}}_{\tau}^{T}+\mathbf{R}
\end{array}\right]\right) \\
& =\mathcal{N}\left(\left[\begin{array}{c}
\mathcal{X} \\
\mathbf{z}
\end{array}\right] \mid\left[\begin{array}{c}
\hat{\mathcal{X}} \\
\mathbf{H} \hat{\mathbf{x}}_{\tau}
\end{array}\right],\left[\begin{array}{cc}
\mathbf{P} & \mathbf{P} \cdot{ }_{\tau} \mathbf{H}^{T} \\
\mathbf{H} \mathbf{P}_{\tau} . & \mathbf{H} \mathbf{P}_{\tau} \mathbf{H}^{T}+\mathbf{R}
\end{array}\right]\right) .
\end{aligned}
$$

where $\overline{\mathbf{H}}_{\tau}$ is a sparse matrix defined such that $\overline{\mathbf{H}}_{\tau} \mathcal{X}=\mathbf{H} \mathbf{x}_{\tau}$. The covariance between state $\mathbf{x}_{i}$ and $\mathbf{x}_{j}$ is denoted $\mathbf{P}_{i, j}$, with the shorthand $\mathbf{P}_{i}=\mathbf{P}_{i, i}$, and $\mathbf{P}{ }_{\tau}$ and $\mathbf{P}_{\tau}$. are the columns and rows in $\mathbf{P}$ corresponding to the state $\mathbf{x}_{\tau}$, respectively. Also, let $\mathbf{S}_{\tau}=\mathbf{H} \mathbf{P}_{\tau} \mathbf{H}^{T}+\mathbf{R}$ for simpler notation. Given an observation $\mathbf{z}$ at time $\tau$ the conditional distribution of $\mathcal{X}$ is given by [63]

$$
\begin{aligned}
p(\mathcal{X} \mid \mathbf{z}, \mathcal{Y}) & =\mathcal{N}\left(\mathcal{X} \mid \hat{\mathcal{X}}+\mathbf{P} \cdot{ }_{\tau} \mathbf{H}^{T} \mathbf{S}_{\tau}^{-1}\left(\mathbf{z}-\mathbf{H} \hat{\mathbf{x}}_{\tau}\right), \mathbf{P}-\mathbf{P} \cdot{ }_{\tau} \mathbf{H}^{T} \mathbf{S}_{\tau}^{-1} \mathbf{H} \mathbf{P}_{\tau} \cdot\right) \\
& =\mathcal{N}\left(\mathcal{X} \mid \hat{\mathcal{X}}^{+}, \mathbf{P}^{+}\right),
\end{aligned}
$$

where the superscript + denotes the conditional quantity.

The full conditional distribution in (4.13) can be broken down into marginal distributions and cross-covariances for individual states as

$$
\begin{aligned}
\hat{\mathbf{x}}_{i}^{+} & =\hat{\mathbf{x}}_{i}+\mathbf{P}_{i, \tau} \mathbf{H}^{T} \mathbf{S}_{\tau}^{-1}\left(\mathbf{z}-\mathbf{H} \hat{\mathbf{x}}_{\tau}\right), \\
\mathbf{P}_{i, j}^{+} & =\mathbf{P}_{i, j}-\mathbf{P}_{i, \tau} \mathbf{H}^{T} \mathbf{S}_{\tau}^{-1} \mathbf{H} \mathbf{P}_{\tau, j} .
\end{aligned}
$$

These equations will serve as a basis for computing the marginal conditional distribution. The covariance matrix $\mathbf{P}$ is assumed to have the structure described in Assumption A4.1. This assumption will be shown to hold for the LGSSM. 
Assumption A4.1. The cross-covariance $\mathbf{P}_{i, j}=\operatorname{Cov}\left(\mathbf{x}_{i}, \mathbf{x}_{j}\right)$ between two states $\mathbf{x}_{i}$ and $\mathbf{x}_{j}$, for $i<j$ is given by

$$
\mathbf{P}_{i, j}=\mathbf{P}_{i, i+1} \mathbf{P}_{i+1}^{-1} \mathbf{P}_{i+1, j}
$$

Through expansion of Assumption A4.1 it follows that

$$
\mathbf{P}_{i, j}=\mathbf{P}_{i, i+1} \prod_{k=i+1}^{j-1} \mathbf{P}_{k}^{-1} \mathbf{P}_{k, k+1}=\mathbf{P}_{i, j-1} \mathbf{P}_{j-1}^{-1} \mathbf{P}_{j-1, j},
$$

and using the symmetry of the covariance matrix, $\mathbf{P}_{i, j}^{T}=\mathbf{P}_{j, i}$, similar results for $i>j$ are obtained by transposing (4.15) and (4.16).

The implication of Assumption A4.1 is that, given the covariance of each state and the cross-covariance of consecutive states, the cross-covariance between any states can be determined. To incorporate the assumption into (4.14), the conditional distribution for $\mathbf{x}_{\tau}$ is obtained as

$$
\begin{aligned}
& \hat{\mathbf{x}}_{\tau}^{+}=\hat{\mathbf{x}}_{\tau}+\mathbf{P}_{\tau} \mathbf{H}^{T} \mathbf{S}_{\tau}^{-1}\left(\mathbf{z}-\mathbf{H} \hat{\mathbf{x}}_{\tau}\right), \\
& \mathbf{P}_{\tau}^{+}=\mathbf{P}_{\tau}-\mathbf{P}_{\tau} \mathbf{H}^{T} \mathbf{S}_{\tau}^{-1} \mathbf{H} \mathbf{P}_{\tau} .
\end{aligned}
$$

The conditional distribution for the states $\mathbf{x}_{k}$ and cross-covariances $\mathbf{P}_{k, k+1}$ between consecutive states for $k<\tau$ are derived as

$$
\begin{aligned}
\hat{\mathbf{x}}_{k}^{+} & =\hat{\mathbf{x}}_{k}+\mathbf{P}_{k, \tau} \mathbf{H}^{T} \mathbf{S}^{-1}\left(\mathbf{z}-\mathbf{H} \hat{\mathbf{x}}_{\tau}\right) \\
& =\hat{\mathbf{x}}_{k}+\mathbf{P}_{k, k+1} \mathbf{P}_{k+1}^{-1} \mathbf{P}_{k+1, \tau} \mathbf{H}^{T} \mathbf{S}^{-1}\left(\mathbf{z}-\mathbf{H} \hat{\mathbf{x}}_{\tau}\right) \\
& =\hat{\mathbf{x}}_{k}-\mathbf{P}_{k, k+1} \mathbf{P}_{k+1}^{-1}\left(\hat{\mathbf{x}}_{k+1}-\hat{\mathbf{x}}_{k+1}^{+}\right), \\
\mathbf{P}_{k, k+1}^{+} & =\mathbf{P}_{k, k+1}-\mathbf{P}_{k, \tau} \mathbf{H}^{T} \mathbf{S}^{-1} \mathbf{H} \mathbf{P}_{\tau, k+1} \\
& =\mathbf{P}_{k, k+1}-\mathbf{P}_{k, k+1} \mathbf{P}_{k+1}^{-1} \mathbf{P}_{k+1, \tau} \mathbf{H}^{T} \mathbf{S}^{-1} \mathbf{H} \mathbf{P}_{\tau, k+1} \\
& =\mathbf{P}_{k, k+1}-\mathbf{P}_{k, k+1} \mathbf{P}_{k+1}^{-1}\left(\mathbf{P}_{k+1}-\mathbf{P}_{k+1}^{+}\right) \\
& =\mathbf{P}_{k, k+1} \mathbf{P}_{k+1}^{-1} \mathbf{P}_{k+1}^{+},
\end{aligned}
$$

and

$$
\begin{aligned}
\mathbf{P}_{k}^{+} & =\mathbf{P}_{k}-\mathbf{P}_{k, \tau} \mathbf{H}^{T} \mathbf{S}^{-1} \mathbf{H} \mathbf{P}_{\tau, k} \\
& =\mathbf{P}_{k}-\mathbf{P}_{k, k+1} \mathbf{P}_{k+1}^{-1} \mathbf{P}_{k+1, \tau} \mathbf{H}^{T} \mathbf{S}^{-1} \mathbf{H} \mathbf{P}_{\tau, k+1} \mathbf{P}_{k+1}^{-1} \mathbf{P}_{k+1, k} \\
& =\mathbf{P}_{k}-\left(\mathbf{P}_{k, k+1}-\mathbf{P}_{k, k+1}^{+}\right) \mathbf{P}_{k+1}^{-1} \mathbf{P}_{k, k+1}^{T},
\end{aligned}
$$

using Assumption A4.1. By denoting $\mathbf{K}_{k}^{b}=\mathbf{P}_{k, k+1} \mathbf{P}_{k+1}^{-1}$ the equations are simplified to

$$
\begin{aligned}
\hat{\mathbf{x}}_{k}^{+} & =\hat{\mathbf{x}}_{k}+\mathbf{K}_{k}^{b}\left(\hat{\mathbf{x}}_{k+1}^{+}-\hat{\mathbf{x}}_{k+1}\right), \\
\mathbf{P}_{k, k+1}^{+} & =\mathbf{P}_{k, k+1}+\mathbf{K}_{k}^{b}\left(\mathbf{P}_{k+1}^{+}-\mathbf{P}_{k+1}\right), \\
\mathbf{P}_{k}^{+} & =\mathbf{P}_{k}+\left(\mathbf{P}_{k, k+1}^{+}-\mathbf{P}_{k, k+1}\right)\left(\mathbf{K}_{k}^{b}\right)^{T},
\end{aligned}
$$


and similar derivations for $k>\tau$ gives, with $\mathbf{K}_{k}^{f}=\mathbf{P}_{k, k-1} \mathbf{P}_{k-1}^{-1}$,

$$
\begin{aligned}
\hat{\mathbf{x}}_{k}^{+} & =\hat{\mathbf{x}}_{k}+\mathbf{K}_{k}^{f}\left(\hat{\mathbf{x}}_{k-1}^{+}-\hat{\mathbf{x}}_{k-1}\right), \\
\mathbf{P}_{k-1, k}^{+} & =\mathbf{P}_{k-1, k}+\left(\mathbf{P}_{k-1}^{+}-\mathbf{P}_{k-1}\right)\left(\mathbf{K}_{k}^{f}\right)^{T}, \\
\mathbf{P}_{k}^{+} & =\mathbf{P}_{k}+\mathbf{K}_{k}^{f}\left(\mathbf{P}_{k-1, k}^{+}-\mathbf{P}_{k-1, k}\right) .
\end{aligned}
$$

Note that the update of the marginal posterior distribution only depends on the cross-covariances between consecutive states and not the full state covariance. As a result, only the state means, state covariances and cross-covariances between consecutive states are needed to compute the marginal posterior distribution.

The conditional cross-covariances in (4.14b) are for $j \leq \tau$ and $i<j$ obtained as

$$
\begin{aligned}
\mathbf{P}_{i, j}^{+} & =\mathbf{P}_{i, j}-\mathbf{P}_{i, \tau} \mathbf{H}^{T} \mathbf{S}_{\tau}^{-1} \mathbf{H} \mathbf{P}_{\tau, j}= \\
& =\mathbf{P}_{i, i+1} \mathbf{P}_{i+1}^{-1} \mathbf{P}_{i+1, j}-\mathbf{P}_{i, i+1} \mathbf{P}_{i+1}^{-1} \mathbf{P}_{i+1, \tau} \mathbf{H}^{T} \mathbf{S}^{-1} \mathbf{H} \mathbf{P}_{\tau, j} \\
& =\mathbf{P}_{i, i+1} \mathbf{P}_{i+1}^{-1} \mathbf{P}_{i+1, j}+\mathbf{P}_{i, i+1} \mathbf{P}_{i+1}^{-1}\left(\mathbf{P}_{i+1, j}^{+}-\mathbf{P}_{i+1, j}\right) \\
& =\mathbf{P}_{i, i+1} \mathbf{P}_{i+1}^{-1} \mathbf{P}_{i+1, j}^{+}=\mathbf{P}_{i, i+1}^{+}\left(\mathbf{P}_{i+1}^{+}\right)^{-1} \mathbf{P}_{i+1, j}^{+},
\end{aligned}
$$

using (4.18b) in the last equality, and for $j>\tau$ and $i<j$ as

$$
\begin{aligned}
\mathbf{P}_{i, j}^{+} & =\mathbf{P}_{i, j}-\mathbf{P}_{i, \tau} \mathbf{H}^{T} \mathbf{S}_{\tau}^{-1} \mathbf{H} \mathbf{P}_{\tau, j}= \\
& =\mathbf{P}_{i, j-1} \mathbf{P}_{j-1}^{-1} \mathbf{P}_{j-1, j}-\mathbf{P}_{i, \tau} \mathbf{H}^{T} \mathbf{S}^{-1} \mathbf{H} \mathbf{P}_{\tau, j-1} \mathbf{P}_{j-1}^{-1} \mathbf{P}_{j-1, j} \\
& =\mathbf{P}_{i, j-1}^{+} \mathbf{P}_{j-1}^{-1} \mathbf{P}_{j-1, j}=\mathbf{P}_{i, j-1}^{+}\left(\mathbf{P}_{j-1}^{+}\right)^{-1} \mathbf{P}_{j-1, j}^{+},
\end{aligned}
$$

using the special case of $i=j-1$ to obtain the last equality, which are equivalent to (4.15) and (4.16), respectively, showing that assumption A4.1 still holds for the conditional distribution.

Considering an LGSSM defined by (2.8), with initial estimate $\mathbf{x}_{0} \sim \mathcal{N}\left(\overline{\mathbf{x}}_{0}, \mathbf{P}_{0}\right)$ and no measurements, the covariance between states $\mathbf{x}_{i}$ and $\mathbf{x}_{j}$ for $i<j$ is given by

$$
\begin{aligned}
\mathbf{P}_{i, j} & =\operatorname{Cov}\left(\mathbf{x}_{i}, \mathbf{x}_{j}\right)=\mathbf{E}\left(\left(\mathbf{x}_{i}-\hat{\mathbf{x}}_{i}\right)\left(\mathbf{x}_{j}-\hat{\mathbf{x}}_{j}\right)^{T}\right) \\
& =\mathbf{E}\left(\left(\mathbf{x}_{i}-\hat{\mathbf{x}}_{i}\right)\left(\mathbf{F}_{j}\left(\mathbf{x}_{j-1}-\hat{\mathbf{x}}_{j-1}\right)+\mathbf{v}_{j}\right)^{T}\right) \\
& =\mathbf{E}\left(\left(\mathbf{x}_{i}-\hat{\mathbf{x}}_{i}\right)\left(\mathbf{x}_{j-1}-\hat{\mathbf{x}}_{j-1}\right)^{T}\right) \mathbf{F}_{j}^{T}=\mathbf{P}_{i, j-1} \mathbf{F}_{j}^{T} \\
& =\mathbf{P}_{i, j-1} \mathbf{P}_{j-1}^{-1} \mathbf{P}_{j-1} \mathbf{F}_{j}^{T}=\mathbf{P}_{i, j-1} \mathbf{P}_{j-1}^{-1} \mathbf{P}_{j-1, j},
\end{aligned}
$$

which shows that Assumption A4.1 holds initially before conditioning on measurements.

Putting together (4.19) and (4.20) results in a method, described in Algorithm 4.5, for efficiently updating a smoothing posterior distribution with an additional measurement $\mathbf{z}$ at arbitrary time $\tau$. A previously added measurement is easily removed by adding it once more with a negative measurement noise covariance matrix. 
The main advantage of this approach is that only one pass is required to update the smoothing posterior distribution compared to two passes required for the forward and backward recursions of the RTSS.

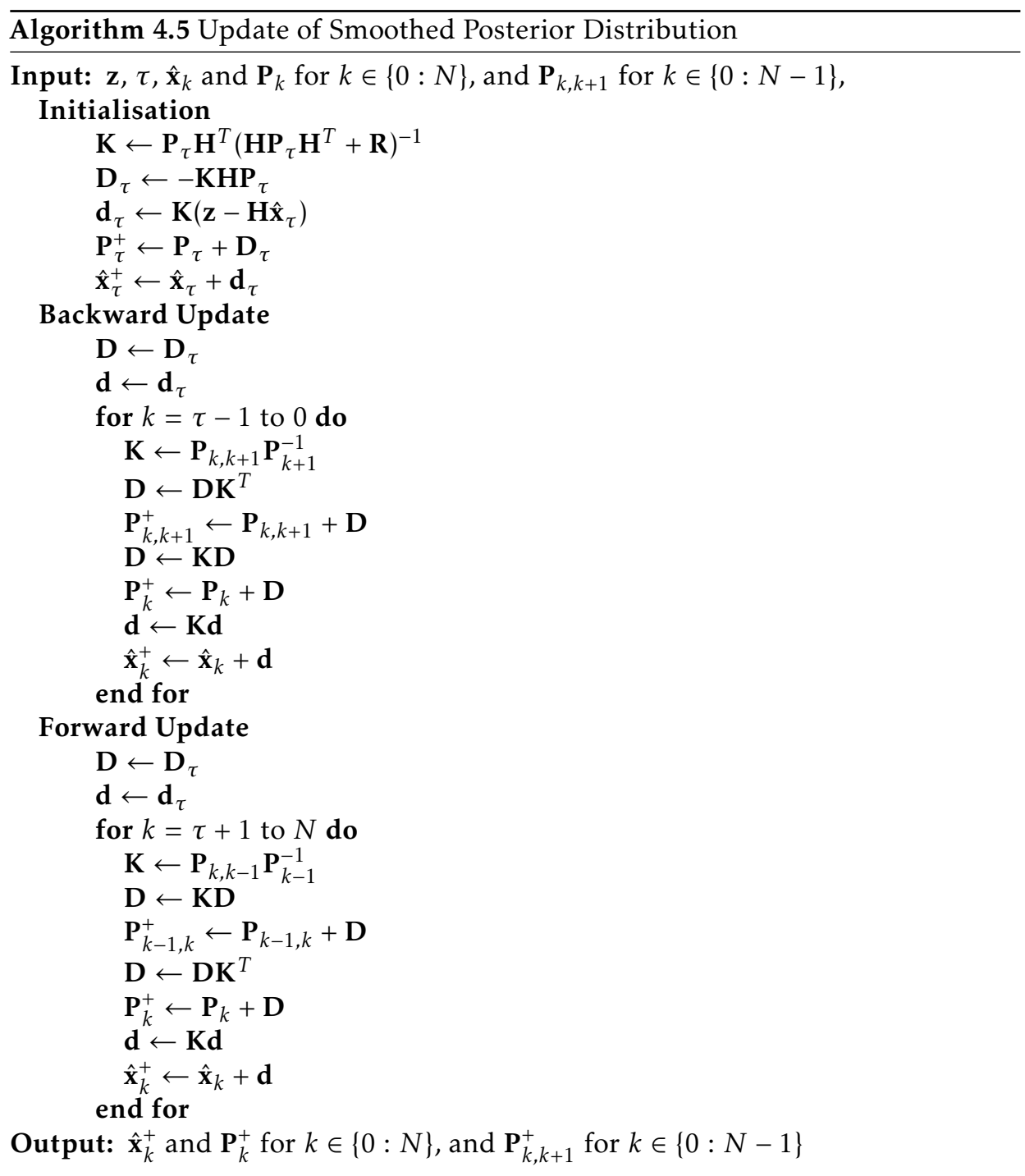

\subsection{Filter Bank}

A method for computing the filtering distribution of a JMM is presented in this section, described in e.g. [63]. A combinatoric explosion occurs when considering all possible mode switches, so pruning of unlikely mode sequences is required for tractability. The filter bank for the JMM is described in Section 4.4.1. The 
modifications required for estimation in the extension of the JMM proposed in Section 2.5.2 is described in Section 4.4.2, which was previously published by the author in [23].

\subsubsection{Filter Bank for the General Model}

Given the model in (2.43), one is interested in the posterior distribution of the states $\mathbf{x}_{k}$ and modes $\delta_{k}, k \in \mathcal{K}$. For a given mode sequence the model reduces to an AWGNSSM and the filtering and smoothing posterior distributions can be approximately computed using, e.g., Algorithms 4.2 and 4.4, respectively.

When the mode sequence $\Delta=\left\{\delta_{k}\right\}_{k \in \mathcal{K}}$ is unknown the posterior is a mixture of $|\mathcal{S}|^{N}$ distributions. By indexing each unique sequence $\Delta_{1: k}^{i}=\left\{\delta_{j}^{i}\right\}_{j=1}^{k}, i \in\left\{1:|\mathcal{S}|^{k}\right\}$, the posterior distribution can be computed as

$$
p\left(\mathbf{x}_{k} \mid \mathcal{Y}_{1: k}\right)=\sum_{i=1}^{|\mathcal{S}|^{k}} p\left(\mathbf{x}_{k}, \Delta_{1: k}^{i} \mid \mathcal{Y}_{1: k}\right)=\sum_{i=1}^{|\mathcal{S}|^{k}} p\left(\Delta_{1: k}^{i} \mid \mathcal{Y}_{1: k}\right) p\left(\mathbf{x}_{k} \mid \Delta_{1: k}^{i}, \mathcal{Y}_{1: k}\right) .
$$

The weight for each distribution in the mixture is computed recursively using

$$
\begin{aligned}
w_{k}^{i} & \triangleq p\left(\Delta_{1: k}^{i} \mid \mathcal{Y}_{1: k}\right)=\frac{p\left(\Delta_{1: k}^{i}\right) p\left(\mathcal{Y}_{1: k} \mid \Delta_{1: k}^{i}\right)}{p\left(\mathcal{Y}_{1: k}\right)} \\
& =\frac{p\left(\Delta_{1: k-1}^{i}\right) p\left(\mathcal{Y}_{1: k-1} \mid \Delta_{1: k}^{i}\right)}{p\left(\mathcal{Y}_{1: k-1}\right)} \cdot p\left(\delta_{k}^{i} \mid \delta_{k-1}^{i}\right) \cdot \frac{p\left(\mathbf{y}_{k} \mid \mathcal{Y}_{1: k-1}, \Delta_{1: k}^{i}\right)}{p\left(\mathbf{y}_{k} \mid \mathcal{Y}_{1: k-1}\right)} \\
& =w_{k-1}^{i} \Pi_{k}^{\delta_{k}^{i}, \delta_{k-1}^{i}} \frac{\int_{\mathbf{x}_{k}} p\left(\mathbf{y}_{k} \mid \mathbf{x}_{k}, \delta_{k}^{i}\right) p\left(\mathbf{x}_{k} \mid \mathcal{Y}_{1: k-1}, \Delta_{1: k}^{i}\right) d \mathbf{x}_{k}}{p\left(\mathbf{y}_{k} \mid \mathcal{Y}_{1: k-1}\right)}
\end{aligned}
$$

where the Markov property of both the mode and the SSM is used. For the LGSSM, this reduces to

$$
\begin{aligned}
w_{k}^{i} & \propto w_{k-1}^{i} \boldsymbol{\Pi}_{k}^{\delta_{k}^{i}, \delta_{k-1}^{i}} \mathcal{N}\left(\mathbf{y}_{k} \mid \mathbf{H}_{k}^{\delta_{k}^{i}} \hat{\mathbf{x}}_{k \mid k-1}^{\Delta_{1: k}^{i}}, \mathbf{H}_{k}^{\delta_{k}^{i}} \mathbf{P}_{k \mid k-1}^{\Delta_{1: k}^{i}} \mathbf{H}_{k}^{\delta_{k}^{i}}+\mathbf{R}_{k}\left(\delta_{k}^{i}\right)\right) \\
& =w_{k-1}^{i} \boldsymbol{\Pi}_{k}^{\delta_{k}^{i}, \delta_{k-1}^{i}} \mathcal{N}\left(\mathbf{y}_{k} \mid \hat{\mathbf{y}}_{k}^{i}, \mathbf{S}_{k}^{i}\right),
\end{aligned}
$$

where $\mathbf{h}_{k}\left(\mathbf{x}_{k}, \delta_{k}\right)=\mathbf{H}_{k}^{\delta_{k}} \mathbf{x}_{k}$, and $p\left(\mathbf{x}_{k} \mid \mathcal{Y}_{1: k-1}, \Delta_{1: k}\right)=\mathcal{N}\left(\mathbf{x}_{k} \mid \hat{\mathbf{x}}_{k \mid k-1}^{\Delta_{1: k}}, \mathbf{P}_{k \mid k-1}^{\Delta_{1: k}}\right)$ is obtained using the $\mathrm{KF}$ in Algorithm 4.2 and the filtering posterior distribution results in a mixture of Gaussians.

Due to the number of distributions in the mixture, it is intractable to compute the complete posterior distribution. Often in practice, only the distributions with the highest weights are computed in real-time applications, or, when all measurements are available, the EM algorithm [16, 40, 83] or Markov Chain Monte Carlo methods [19] can be used to approximate the posterior distribution.

A common method for real-time applications is to view the growing number of unique sequences as a tree and prune or merge low-probability branches [26]. A method that keeps the $M$ most likely branches by pruning is presented in Algorithm 4.6. The main idea is that old high-probability branches are extended with 
all possible modes for the current time step in a sequence-splitting step. A time update is performed for all branches and the prediction is evaluated against the current measurement to compute a new weight for the branch. Low-probability branches are then discarded and the a measurement update is performed for the remaining branches. The KF can suboptimally be replaced by the EKF or IEKF for the AWGNSSM.

Depending on the application one might also be interested in the mode probability, which can be approximated as

$$
p\left(\delta_{k} \mid \mathcal{Y}_{1: k}\right) \approx \sum_{i \in \mathcal{I}_{k}} w_{k}^{i},
$$

where $\mathcal{I}_{k}=\left\{i \in\{1: M\} \mid \delta_{k}^{i}=\delta_{k}\right\}$, or the state, which can be estimated as

$$
\hat{\mathbf{x}}_{k}=\sum_{i=1}^{M} w_{k}^{i} \hat{\mathbf{x}}_{k \mid k}^{i} .
$$

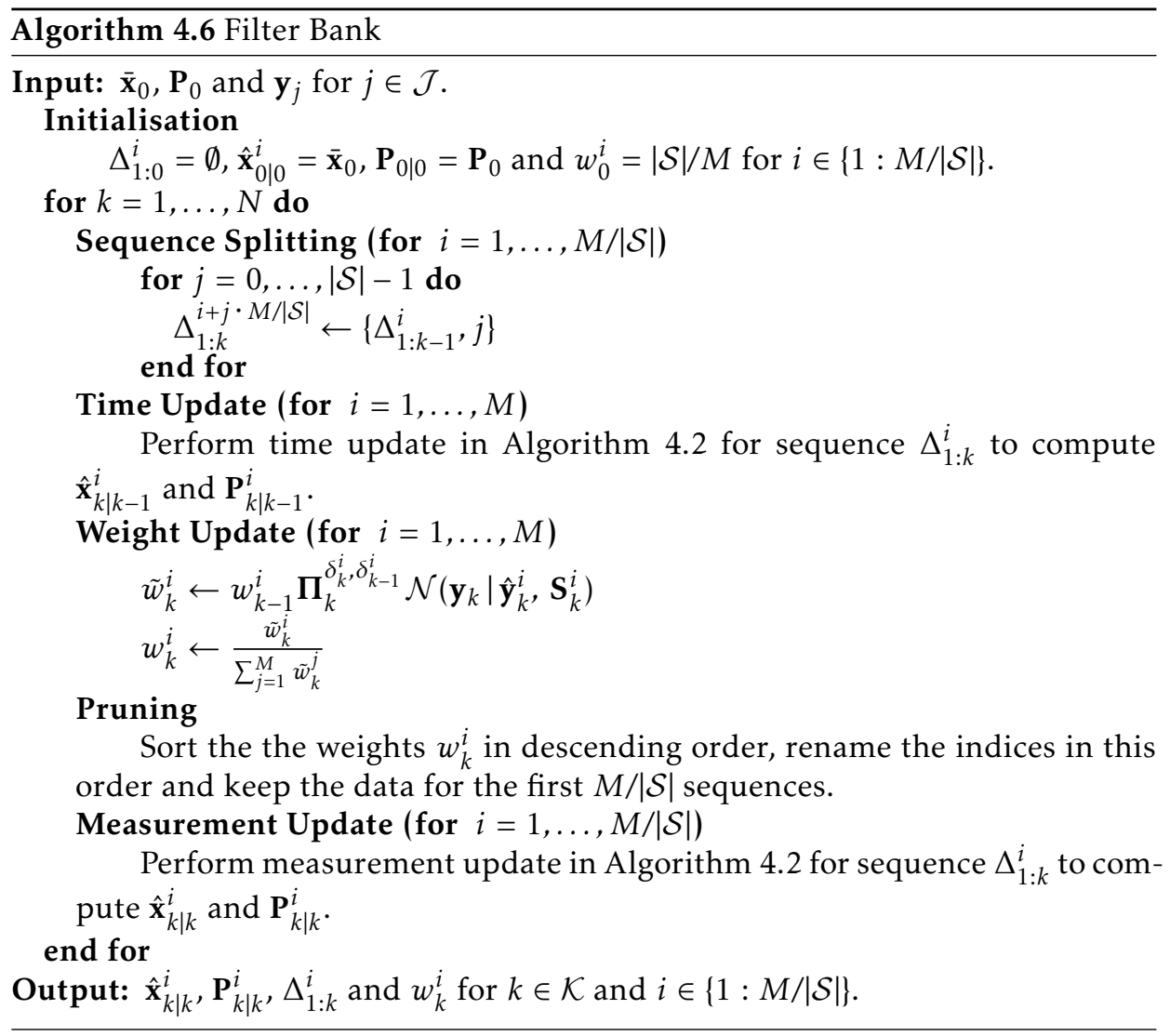




\subsubsection{Filter Bank for the Model Extension}

The modifications required for estimation in the extension of the JMM proposed in Section 2.5.2 is presented in this section. The modifications were previously published by the author in [23].

The main difference to the computations in Section 4.4 when adding the additional measurement in (2.46) is that the weight computation becomes

$$
\begin{aligned}
& w_{k}^{i} \triangleq p\left(\Delta_{1: k}^{i} \mid \mathcal{Y}_{1: k}, \mathcal{Z}_{1: k}\right)=\frac{p\left(\Delta_{1: k}^{i}\right) p\left(\mathcal{Y}_{1: k}, \mathcal{Z}_{1: k} \mid \Delta_{1: k}^{i}\right)}{p\left(\mathcal{Y}_{1: k}, \mathcal{Z}_{1: k}\right)} \\
&= \frac{p\left(\Delta_{1: k-1}^{i}\right) p\left(\mathcal{Y}_{1: k-1}, \mathcal{Z}_{1: k-1} \mid \Delta_{1: k}^{i}\right)}{p\left(\mathcal{Y}_{1: k-1}, \mathcal{Z}_{1: k-1}\right)} \cdot p\left(\delta_{k}^{i} \mid \delta_{k-1}^{i}\right) \\
& \frac{p\left(\mathbf{y}_{k}, \mathbf{z}_{k} \mid \mathcal{Y}_{1: k-1}, \mathcal{Z}_{1: k-1}, \Delta_{1: k}^{i}\right)}{p\left(\mathbf{y}_{k}, \mathbf{z}_{k} \mid \mathcal{Y}_{1: k-1}, \mathcal{Z}_{1: k-1}\right)} \\
&=w_{k-1}^{i} \Pi_{k}^{\delta_{k}^{i}, \delta_{k-1}^{i}} \frac{p\left(\mathbf{z}_{k} \mid \delta_{k}^{i}\right) \int_{\mathbf{x}_{k}} p\left(\mathbf{y}_{k} \mid \mathbf{x}_{k}, \delta_{k}^{i}\right) p\left(\mathbf{x}_{k} \mid \mathcal{Y}_{1: k-1}, \Delta_{1: k}^{i}\right) d \mathbf{x}_{k}}{p\left(\mathbf{y}_{k}, \mathbf{z}_{k} \mid \mathcal{Y}_{1: k-1}, \mathcal{Z}_{1: k-1}\right)}
\end{aligned}
$$

adding another factor to the weight-update product. For the LGSSM the computation reduces to

$$
w_{k}^{i} \propto w_{k-1}^{i} \boldsymbol{\Pi}_{k}^{\delta_{k}^{i}, \delta_{k-1}^{i}} \mathcal{N}\left(\mathbf{y}_{k} \mid \hat{\mathbf{y}}_{k}^{i}, \mathbf{S}_{k}^{i}\right) p\left(\mathbf{z}_{k} \mid \delta_{k}^{i}\right)
$$

The proposed extension is used for estimating the bird takeoff times as presented in Application 4.1. The results for the application are presented as well, with a comparison to a sequence of manually annotated data.

\section{Application 4.1: Bird Mode Estimation}

The main goal is to determine time and direction of takeoffs, and the secondary goal is to measure the activity of the bird. The time of a takeoff is estimated as a transition from stationary to flight in the JMM in Application 2.8 and the direction is estimated from the states in the JMM.

Together with the models described in Applications 2.8, 2.9 and 3.1 the methods described in this chapter are used to estimate both the current mode of the bird and its position within the funnel, providing sufficient information to extract time and direction of the takeoffs.

An assay was prepared filming four European robins over approximately an hour at six frames per second. Using the image processing methods described in Application 3.4, the position, orientation and blurriness of the birds are extracted. The camera model is estimated for each funnel according to the method described in Application 3.1. Two models are set up as in Applications 2.8 and 2.9, one for the stationary mode with low process noise covariance, low blur level and high probability of being close to the centre of the funnel, and another for the flight mode with opposite characteristics.

The method described in this section with an IEKF is used to estimate the states 
and modes for the birds. The advantage of using the IEKF is mainly that the position of the bird in world coordinates is more accurate compared to an EKF. The estimated mode over time for each bird is shown in Figure 4.1. The estimation is at times noisy, so some heuristics are applied to filter out short mode intervals and movements that are too small to be takeoffs. Each transition from stationary to flight mode is then extracted as a takeoff, shown in Figure 4.2.

The direction is extracted by a basic method, computed as the angle between the position before and after a transition. Due to the low frame rate, however, the particular frames might not be representative of the takeoff. An improved method therefore considers the position of the bird in a number of frames around the transition where the angle of the trajectory only changes by a reasonable amount. A third, simpler, method that does not require a filter is also considered for comparison. In this method a takeoff is estimated every time the bird leaves the inner circle. The direction is extracted as the angle between the estimated position outside the inner circle and the centre of the funnel.

Figure 4.3 shows the result of the considered methods, as well as an average takeoff direction, where all methods result in similar averages. Figure 4.4 shows an example of a takeoff drawn into the original video using the improved method.

The proposed method is compared to a traditional method and some modern methods in [23], and is deemed to be a viable solution to automate the biological experiment. In addition to providing similar data as traditional methods, the proposed method also provides a temporal dimension and experiments can be conducted for a longer time without disturbing the birds. An additional advantage is that the birds can potentially be held in larger cages with food and water, allowing, from an ethical point of view, for even longer experiments. The biologists are also interested in the activity of the birds, which can, e.g., be extracted from the estimated modes or from the estimated states by computing the distance travelled by the bird.

The first quarter of the experiment has been manually annotated, so the time and direction of all takeoffs during this period are available. The takeoffs are in this case defined slightly differently as the maximum distance of the beak from the funnel centre in image coordinates, making a comparison of the orientations for individual takeoffs less informative. A disadvantage of the manual method is that when a bird pursues a takeoff for a long time, bouncing against the net, it is likely to result in an estimated takeoff direction that was initially not intended by the bird. However, takeoff times should not differ too much for individual takeoffs and the average takeoff directions should be comparable between the methods.

A takeoff time estimated using the proposed method is matched to a takeoff in the manually annotated data if the time difference is low. The results for matching takeoff times between the methods are shown in Table 4.1. A large percentage of all takeoffs detected by the proposed method are also annotated manually, but less than half of the manually annotated takeoffs are detected by the proposed method. The results are better, however, if the fourth cage is disregarded. The 

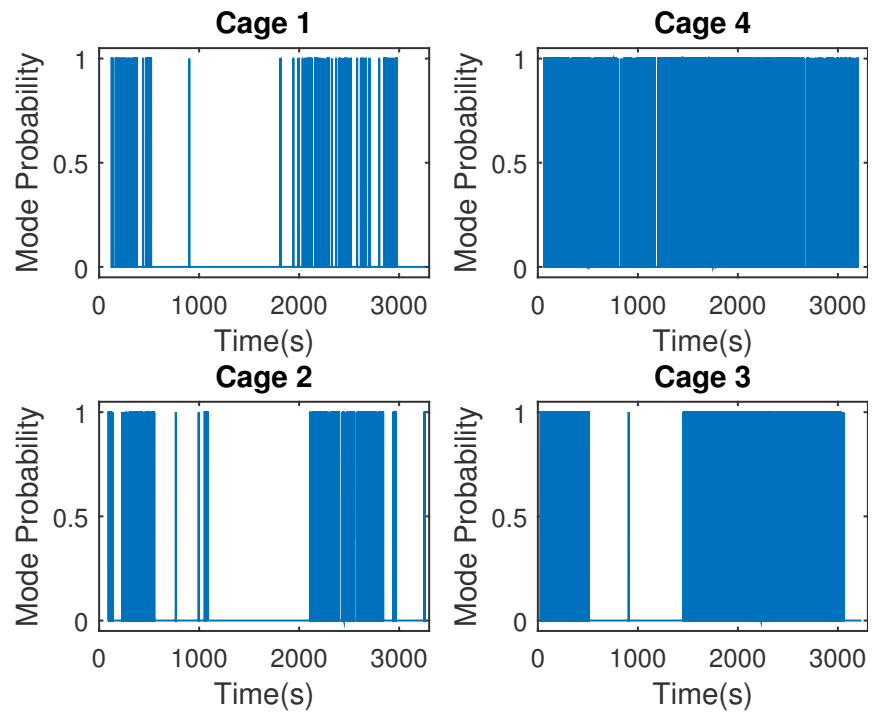

Figure 4.1: Mode probability for flight mode over time for all four birds.

Cage 1

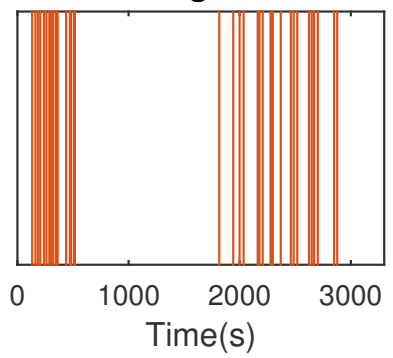

Cage 2

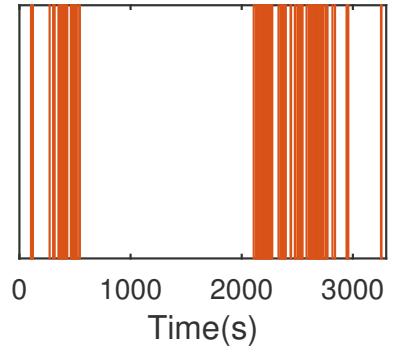

Cage 4

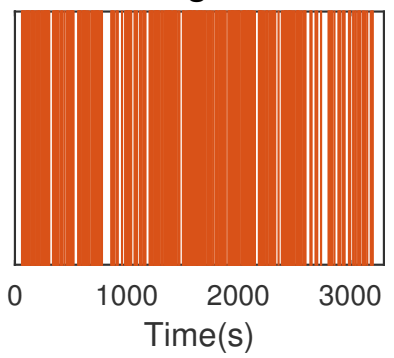

Cage 3

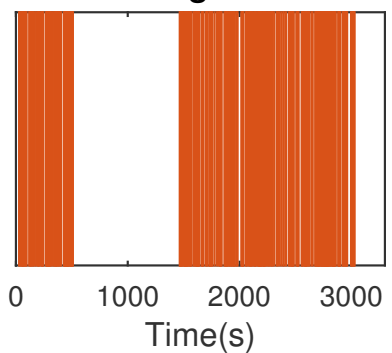

Figure 4.2: Estimated times of takeoffs for all four birds after heuristic filtering. 

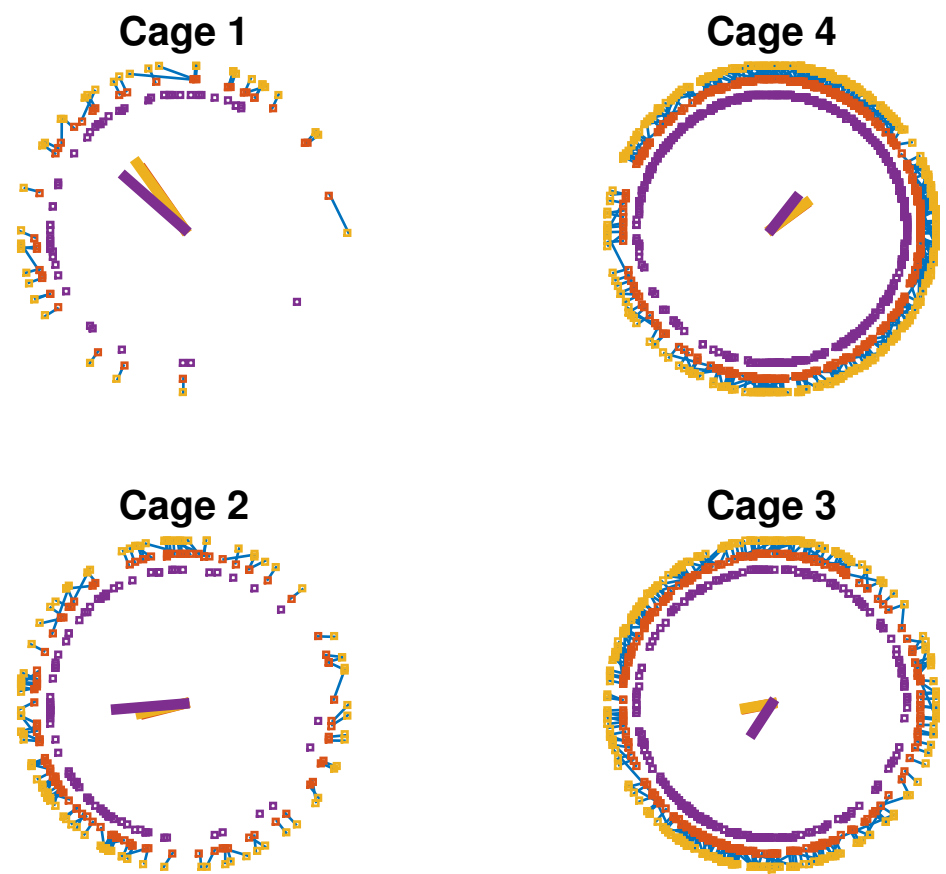

Figure 4.3: The estimates for the basic and improved methods for extracting takeoff directions are shown as the outer and the middle dots, respectively, with a line connecting dots extracted from the same takeoff. The inner circle shows the direction from the centre in which the bird left the inner circle, completely disregarding the mode estimates. The lines in the centre indicate average directions of takeoff for the methods, where a long line is more significant than a short line.
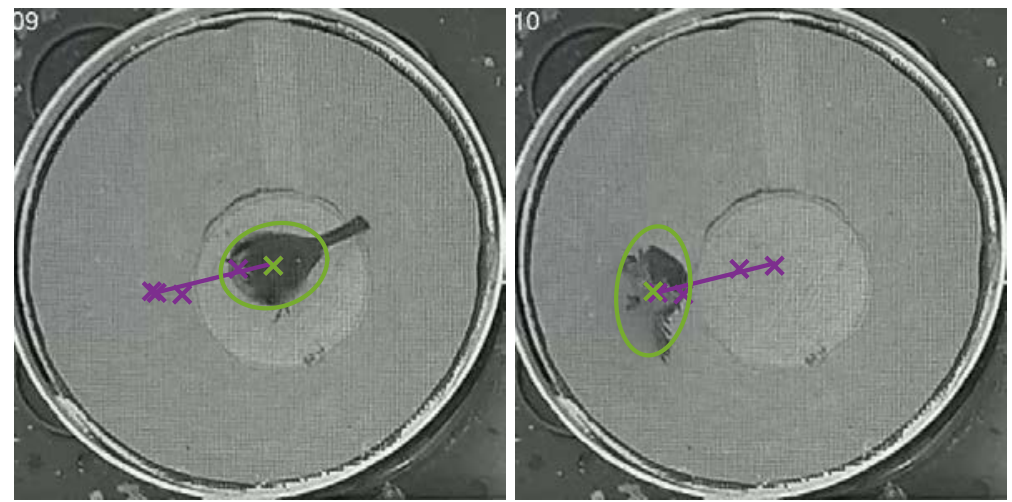

Figure 4.4: A takeoff estimated over several frames using the improved method. Bird position and extent is in green, positions included in the takeoff are magenta and the line is the estimated takeoff direction. 
Table 4.1: In the two right columns are the number of estimated takeoffs for the manual method and the proposed method respectively. In the second column are the number of matches between them. The percentage indicates the proportion matched.

\begin{tabular}{cccc}
\hline Cage & Matches & Manual Method & Proposed Method \\
\hline $\mathbf{1}$ & 14 & $21(67 \%)$ & $18(78 \%)$ \\
$\mathbf{2}$ & 22 & $35(63 \%)$ & $31(71 \%)$ \\
$\mathbf{3}$ & 60 & $87(69 \%)$ & $66(91 \%)$ \\
$\mathbf{4}$ & 42 & $190(22 \%)$ & $51(82 \%)$ \\
\hline All & 138 & $333(41 \%)$ & $166(83 \%)$ \\
\hline
\end{tabular}

fourth cage has particularly many takeoffs due to an active bird, corroborated by Figures 4.1 and 4.2. An active bird spends less time scanning in the centre of the funnel, making it more difficult to separate individual takeoffs. The policy used in the manual method for estimating takeoffs also includes bounces back through the inner circle, which are more likely to occur for an active bird and are of less interest considering the bird did not intentionally choose the direction. Such takeoffs are however discarded by the proposed method, which could be an explanation for the large discrepancy for the fourth cage.

Individual takeoffs that are matched between the methods are shown in Figure 4.5. Since takeoff directions are defined differently, these methods show many severe mismatches in direction, but as can be seen, the average directions, shown as lines in the centre of the circle, are similar between the methods.

\subsection{Extended Target Model Estimation}

As presented in [79], the approach for estimation in the extended target model in Section 2.6 is similar to estimation for the ordinary SSM presented in (4.1) and (4.2). The difference is in an additional consideration for an unknown covariance matrix.

Given measurements $\mathcal{Y}_{1: k}=\left\{\mathcal{Y}_{j}\right\}_{j \in\{1: k\} \cap \mathcal{J}}$ and the model in Section 2.6, the posterior distribution $p\left(\mathbf{x}_{k}, \mathbf{X}_{k} \mid \mathcal{Y}_{1: k}\right)$ of the kinematic state $\mathbf{x}_{k}$ and extent $\mathbf{X}_{k}$ are sought.

Using Bayes' theorem and the Markov property, the time update is given by

$$
\begin{aligned}
& p\left(\mathbf{x}_{k}, \mathbf{X}_{k} \mid \mathcal{Y}_{1: k-1}\right)= \int p\left(\mathbf{x}_{k}, \mathbf{X}_{k} \mid \mathbf{x}_{k-1}, \mathbf{X}_{k-1}, \mathcal{Y}_{k-1}\right) \cdot \\
& p\left(\mathbf{x}_{k-1}, \mathbf{X}_{k-1} \mid \mathcal{Y}_{k-1}\right) \mathrm{d} \mathbf{x}_{k-1} \mathrm{~d} \mathbf{X}_{k-1} \\
&=\int p\left(\mathbf{x}_{k} \mid \mathbf{x}_{k-1}, \mathbf{X}_{k}\right) \cdot p\left(\mathbf{X}_{k} \mid \mathbf{X}_{k-1}\right) \cdot p\left(\mathbf{x}_{k-1} \mid \mathbf{X}_{k-1}, \mathcal{Y}_{k-1}\right) . \\
& p\left(\mathbf{X}_{k-1} \mid \mathcal{Y}_{k-1}\right) \mathrm{d} \mathbf{x}_{k-1} \mathrm{~d} \mathbf{X}_{k-1} .
\end{aligned}
$$


Cage 1

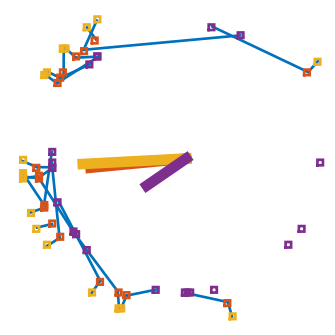

Cage 2

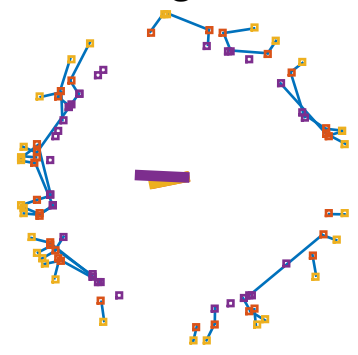

Cage 4

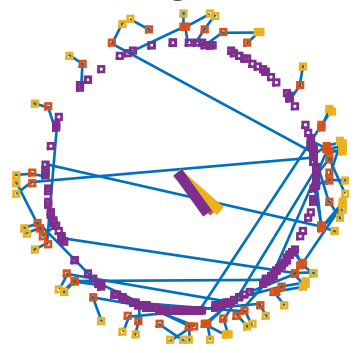

Cage 3

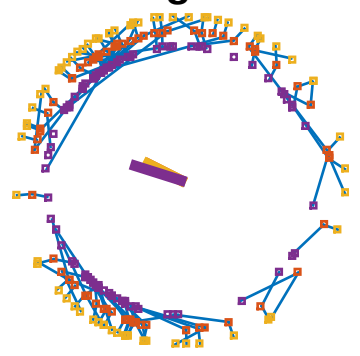

Figure 4.5: Going inwards, the estimated takeoff directions using the basic, improved and manual methods are shown as dots. The connection between the dots indicate that the time of the takeoffs are similar and therefore represent the same takeoff.

and the measurement update by

$$
\begin{aligned}
p\left(\mathbf{x}_{k}, \mathbf{X}_{k} \mid \mathcal{Y}_{1: k}\right) & \propto p\left(\mathcal{Y}_{k}, N_{j} \mid \mathbf{x}_{k}, \mathbf{X}_{k}, \mathcal{Y}_{k-1}\right) \cdot p\left(\mathbf{x}_{k}, \mathbf{X}_{k} \mid \mathcal{Y}_{k-1}\right) \\
& =p\left(\mathcal{Y}_{k} \mid N_{j}, \mathbf{x}_{k}, \mathbf{X}_{k}\right) \cdot p\left(N_{k} \mid \mathbf{x}_{k}, \mathbf{X}_{k}\right) \cdot p\left(\mathbf{x}_{k}, \mathbf{X}_{k} \mid \mathcal{Y}_{k-1}\right) \\
& \propto \prod_{i=1}^{N_{k}} p\left(\mathbf{y}_{k}^{i} \mid \mathbf{x}_{k}, \mathbf{X}_{k}\right) \cdot p\left(\mathbf{x}_{k} \mid \mathbf{x}_{k}, \mathcal{Y}_{k-1}\right) p\left(\mathbf{X}_{k} \mid \mathcal{Y}_{k-1}\right) .
\end{aligned}
$$

All distributions required for (4.31) and (4.32) are given in Section 2.6. Since conjugate prior distributions [53] are chosen for the kinematic state and the extent the posterior distributions can be computed in closed form [79].

The prediction and filtering distributions for the model in (2.51)-(2.55) are computed, using derivations in [79], as

$$
p\left(\mathbf{x}_{k}, \mathbf{X}_{k} \mid \mathcal{Y}_{1: k-1}\right)=\mathcal{N}\left(\mathbf{x}_{k} \mid \hat{\mathbf{x}}_{k \mid k-1}, \mathbf{P}_{k \mid k-1} \otimes \mathbf{X}_{k}\right) \cdot \mathcal{I} \mathcal{W}\left(\mathbf{X}_{k} \mid v_{k \mid k-1}, \mathbf{V}_{k \mid k-1}\right),
$$

and

$$
p\left(\mathbf{x}_{k}, \mathbf{X}_{k} \mid \mathcal{Y}_{1: k}\right)=\mathcal{N}\left(\mathbf{x}_{k} \mid \hat{\mathbf{x}}_{k \mid k}, \mathbf{P}_{k \mid k} \otimes \mathbf{X}_{k}\right) \cdot \mathcal{I} \mathcal{W}\left(\mathbf{X}_{k} \mid v_{k \mid k}, \mathbf{V}_{k \mid k}\right),
$$


where $\mathbf{P}_{k \mid k-1} \in S_{++}^{s}$ and $\mathbf{P}_{k \mid k} \in S_{++}^{s}$. The time update is performed as

$$
\begin{aligned}
\hat{\mathbf{x}}_{k \mid k-1} & =\left(\tilde{\mathbf{F}}_{k} \otimes \mathbf{I}_{p}\right) \hat{\mathbf{x}}_{k-1 \mid k-1}, \\
\mathbf{P}_{k \mid k-1} & =\tilde{\mathbf{F}}_{k} \mathbf{P}_{k-1 \mid k-1} \tilde{\mathbf{F}}_{k}^{T}+\tilde{\mathbf{Q}}_{k}, \\
v_{k \mid k-1} & =e^{-T_{k} / \tau} v_{k-1}, \\
\mathbf{V}_{k \mid k-1} & =\frac{e^{-T_{k} / \tau} v_{k-1 \mid k-1}-2 p-2}{v_{k-1 \mid k-1}-2 p-2} \mathbf{V}_{k-1 \mid k-1},
\end{aligned}
$$

and the measurement update as

$$
\begin{aligned}
\hat{\mathbf{x}}_{k \mid k} & =\hat{\mathbf{x}}_{k \mid k-1}+\left(\tilde{\mathbf{K}}_{k} \otimes \mathbf{I}_{p}\right)\left(\mathbf{y}_{k}-\left(\tilde{\mathbf{H}}_{k} \otimes \mathbf{I}_{p}\right) \hat{\mathbf{x}}_{k \mid k-1}\right), \\
\mathbf{P}_{k \mid k} & =\mathbf{P}_{k \mid k-1}-\tilde{\mathbf{K}}_{k} S_{k} \tilde{\mathbf{K}}_{k}^{T}, \\
v_{k \mid k} & =v_{k \mid k-1}+N_{k}, \\
\mathbf{V}_{k \mid k} & =\mathbf{V}_{k \mid k-1}+\mathbf{N}_{k}+\mathbf{Y}_{k},
\end{aligned}
$$

where

$$
\begin{aligned}
\tilde{\mathbf{K}}_{k} & =\mathbf{P}_{k \mid k-1} \tilde{\mathbf{H}}_{k}^{T} S_{k}^{-1}, \\
S_{k} & =\tilde{\mathbf{H}}_{k} \mathbf{P}_{k \mid k-1} \tilde{\mathbf{H}}_{k}^{T}+\frac{1}{N_{k}}, \\
\mathbf{y}_{k} & =\frac{1}{N_{k}} \sum_{i=1}^{N_{k}} \mathbf{y}_{k}^{i}, \\
\mathbf{Y}_{k} & =\frac{1}{N_{k}} \sum_{i=1}^{N_{k}}\left(\mathbf{y}_{k}^{i}-\mathbf{y}_{k}\right)\left(\mathbf{y}_{k}^{i}-\mathbf{y}_{k}\right)^{T}, \\
\mathbf{N}_{k} & =S_{k}^{-1}\left(\mathbf{y}_{k}-\left(\tilde{\mathbf{H}}_{k} \otimes \mathbf{I}_{p}\right) \hat{\mathbf{x}}_{k \mid k-1}\right)\left(\mathbf{y}_{k}-\left(\tilde{\mathbf{H}}_{k} \otimes \mathbf{I}_{p}\right) \hat{\mathbf{x}}_{k \mid k-1}\right)^{T} .
\end{aligned}
$$

The time and measurement updates for the kinematic state vector are similar to those in the KF. Estimates of the kinematic state covariance and the covariance matrix representing the extent are computed as $[59,79]$

$$
\hat{\mathbf{P}}_{k}=\frac{\mathbf{P}_{k \mid k} \otimes \mathbf{V}_{k \mid k}}{v_{k \mid k}+s-s p-2},
$$

and

$$
\hat{\mathbf{X}}_{k}=\frac{\mathbf{V}_{k \mid k}}{v_{k \mid k}-2 p-2},
$$

for $v_{k \mid k}$ such that the denominators are positive.

The covariance matrix representing the extent is estimated with the assumption that the measurements are Gaussian distributed over the target. However, a uniform distribution is a more reasonable assumption for measurements from a camera sensor due to the uniform sampling grid of the pixels. Assuming that the targets are elliptical and that the measurements are uniformly distributed, the 
elliptical extent is computed as the solutions of $\mathbf{z}$ in the inequality

$$
\left(\mathbf{z}-\left(\mathbf{H}_{k} \otimes \mathbf{I}_{p}\right) \hat{\mathbf{x}}_{k \mid k}\right)^{T} \hat{\mathbf{X}}_{k \mid k}^{-1}\left(\mathbf{z}-\left(\mathbf{H}_{k} \otimes \mathbf{I}_{p}\right) \hat{\mathbf{x}}_{k \mid k}\right) \leq 4
$$

using Proposition 4.1. A simple method to generate a path along the edge is to Cholesky factorise the covariance matrix and perform an affine transformation on a path generated along the unit circle.

Proposition 4.1. Given is a stochastic variable with a uniform distribution

$$
\mathbf{x} \sim p(\mathbf{x})= \begin{cases}A_{\mathcal{C}}^{-1}, & \text { if } \mathbf{x} \in \mathcal{C}, \\ 0, & \text { otherwise }\end{cases}
$$

over an ellipse defined by

$$
\mathcal{C}=\left\{\mathbf{x} \in \mathbb{R}^{2} \mid(\mathbf{x}-\mathbf{b})^{T} \mathbf{C}^{-1}(\mathbf{x}-\mathbf{b}) \leq 1\right\},
$$

where $\mathbf{C}=\mathbf{A A}^{T}$ is a symmetric and positive definite matrix, $\mathbf{b}$ is the centroid of the ellipse and $A_{\mathcal{C}}$ is the area of the ellipse.

The mean and covariance of the stochastic variable are then given by

$$
\mathbf{E}(\mathbf{x})=\mathbf{b} \quad \text { and } \quad \operatorname{Cov}(\mathbf{x})=\frac{\mathbf{C}}{4} .
$$

Proof: The area of the ellipse is given by [64]

$$
A_{\mathcal{C}}=\frac{\pi}{\sqrt{|\mathbf{C}|}}=\pi|\mathbf{A}| \text {. }
$$

The mean of the stochastic variable is computed as

$$
\mathbf{E}(\mathbf{x})=\int \mathbf{x} p(\mathbf{x}) \mathrm{d} \mathbf{x}=\frac{1}{\pi|\mathbf{A}|} \int_{\mathcal{C}} \mathbf{x} \mathrm{d} \mathbf{x} .
$$

Substitution of $\mathbf{x}=\mathbf{A} \tilde{\mathbf{x}}+\mathbf{b}$, with $\mathrm{d} \mathbf{x}=|\mathbf{A}| \mathrm{d} \tilde{\mathbf{x}}$, gives

$$
\mathbf{E}(\mathbf{x})=\frac{1}{\pi|\mathbf{A}|} \int_{\tilde{\mathbf{x}}^{T} \tilde{\mathbf{x}} \leq 1}(\mathbf{A} \tilde{\mathbf{x}}+\mathbf{b})|\mathbf{A}| \mathrm{d} \tilde{\mathbf{x}}=\frac{1}{\pi} \int_{\tilde{\mathbf{x}}^{T} \tilde{\mathbf{x}} \leq 1}(\mathbf{A} \tilde{\mathbf{x}}+\mathbf{b}) \mathrm{d} \tilde{\mathbf{x}} .
$$

Substitution to polar coordinates, $\tilde{\mathbf{x}}=(r \cos (\alpha), r \sin (\alpha))^{T}$ and $\mathrm{d} \tilde{\mathbf{x}}=r \mathrm{~d} r \mathrm{~d} \alpha$, gives

$$
\begin{aligned}
\mathbf{E}(\mathbf{x}) & =\frac{1}{\pi} \int_{0}^{2 \pi} \int_{0}^{1}\left(\mathbf{A}\left(\begin{array}{l}
r \cos (\alpha) \\
r \sin (\alpha)
\end{array}\right)+\mathbf{b}\right) r \mathrm{~d} r \mathrm{~d} \alpha \\
& =\frac{1}{\pi} \mathbf{b} \int_{0}^{2 \pi} \int_{0}^{1} r \mathrm{~d} r \mathrm{~d} \alpha=\frac{1}{\pi} \mathbf{b} \cdot \pi=\mathbf{b} .
\end{aligned}
$$


The covariance of the stochastic variable is computed as

$$
\operatorname{Cov}(\mathbf{x})=\mathbf{E}\left((\mathbf{x}-\mathbf{E}(\mathbf{x}))(\mathbf{x}-\mathbf{E}(\mathbf{x}))^{T}\right)=\frac{1}{\pi|\mathbf{A}|} \int_{\mathcal{C}}(\mathbf{x}-\mathbf{b})(\mathbf{x}-\mathbf{b})^{T} \mathrm{~d} \mathbf{x} .
$$

Substitution of $\mathbf{x}=\mathbf{A} \tilde{\mathbf{x}}+\mathbf{b}$, with $\mathrm{d} \mathbf{x}=|\mathbf{A}| \mathrm{d} \tilde{\mathbf{x}}$, gives

$$
\operatorname{Cov}(\mathbf{x})=\frac{1}{\pi} \int_{\tilde{\mathbf{x}}^{T} \tilde{\mathbf{x}} \leq 1} \mathbf{A}\left(\tilde{\mathbf{x}} \tilde{\mathbf{x}}^{T}\right) \mathbf{A}^{T} \mathrm{~d} \tilde{\mathbf{x}}
$$

Substitution to polar coordinates, $\tilde{\mathbf{x}}=(r \cos (\alpha), r \sin (\alpha))^{T}$ and $\mathrm{d} \tilde{\mathbf{x}}=r \mathrm{~d} r \mathrm{~d} \alpha$, gives

$$
\begin{aligned}
\operatorname{Cov}(\mathbf{x}) & =\frac{1}{\pi} \mathbf{A}\left(\int_{0}^{2 \pi} \int_{0}^{1} r^{3}\left(\begin{array}{cc}
\cos ^{2}(\alpha) & \sin (\alpha) \cos (\alpha) \\
\sin (\alpha) \cos (\alpha) & \sin ^{2}(\alpha)
\end{array}\right) \mathrm{d} r \mathrm{~d} \alpha\right) \mathbf{A}^{T} \\
& =\frac{1}{\pi} \mathbf{A}\left(\frac{1}{4} \cdot \pi \mathbf{I}_{2}\right) \mathbf{A}^{T}=\frac{\mathbf{A} \mathbf{A}^{T}}{4}=\frac{\mathbf{C}}{4} .
\end{aligned}
$$

The method described in this section is applied in the savannah application. However, considering the association uncertainty in the application the presentation is deferred to Application 5.1 in Chapter 5.

\subsection{Uncertain Timestamp Model Estimation}

Conditional on the uncertain timestamp, the UTM defined in Section 2.7 reduces to an LGSSM, for which the states can be estimated using the RTSS [97]. This is used to handle the uncertainty of the timestamp and relevant posterior distributions and estimators are derived in Sections 4.6.1 and 4.6.2, respectively. The contents of this section are considered by the author to be the main contribution in the thesis. The derivations in this section, the results for the simple uncertain time scenario and the analysis in Application 4.7 were previously published by the author in [113].

\subsubsection{Posterior Distributions}

Given the UTM in Section 2.7, the joint posterior distribution of all states is given by marginalization as

$$
p(\mathcal{X} \mid \mathcal{Y}, \mathbf{z})=\sum_{\tau \in \mathcal{K}} p(\mathcal{X}, \tau \mid \mathcal{Y}, \mathbf{z})
$$

where the joint posterior distribution of all states and the uncertain time can be computed up to a constant factor using Bayes' theorem and the independence assumptions of the measurement and transition functions as

$$
p(\mathcal{X}, \tau \mid \mathcal{Y}, \mathbf{z}) \propto p(\tau) p\left(\mathbf{x}_{0}\right) p\left(\mathbf{z} \mid \mathbf{x}_{\tau}, \tau\right) \prod_{j \in \mathcal{J}} p\left(\mathbf{y}_{j} \mid \mathbf{x}_{j}\right) \prod_{k \in \mathcal{K}} p\left(\mathbf{x}_{k} \mid \mathbf{x}_{k-1}\right)
$$




$$
\begin{aligned}
& =p(\tau) \mathcal{N}\left(\mathbf{x}_{0} \mid \overline{\mathbf{x}}_{0}, \mathbf{P}_{0}\right) \mathcal{N}\left(\mathbf{z} \mid \mathbf{H}^{z} \mathbf{x}_{\tau}, \mathbf{R}^{z}\right) \\
& \quad \times \prod_{j \in \mathcal{J}} \mathcal{N}\left(\mathbf{y}_{j} \mid \mathbf{H}_{j}^{y} \mathbf{x}_{j}, \mathbf{R}_{j}^{y}\right) \prod_{k \in \mathcal{K}} \mathcal{N}\left(\mathbf{x}_{k} \mid \mathbf{F}_{k} \mathbf{x}_{k-1}, \mathbf{Q}_{k}\right) \\
& \propto p(\tau) \exp \left[-\frac{1}{2}\left(\| \mathbf{x}_{0}-\overline{\mathbf{x}}_{0}\right)\left\|_{\mathbf{P}_{0}}^{2}+\right\| \mathbf{z}-\mathbf{H}^{z} \mathbf{x}_{\tau} \|_{\mathbf{R}^{z}}^{2}\right. \\
& \left.\left.\quad+\sum_{j \in \mathcal{J}}\left\|\mathbf{y}_{j}-\mathbf{H}_{j}^{y} \mathbf{x}_{j}\right\|_{\mathbf{R}_{j}^{y}}^{2}+\sum_{k \in \mathcal{K}}\left\|\mathbf{x}_{k}-\mathbf{F}_{k} \mathbf{x}_{k-1}\right\|_{\mathbf{Q}_{k}}^{2}\right)\right],
\end{aligned}
$$

where $\|\mathbf{a}\|_{\mathbf{A}}^{2}=\mathbf{a}^{T} \mathbf{A}^{-1} \mathbf{a}$ is the squared Mahalanobis distance.

Alternatively, (4.46) can be factorized as

$$
p(\mathcal{X}, \tau \mid \mathcal{Y}, \mathbf{z})=p(\mathcal{X} \mid \tau, \mathcal{Y}, \mathbf{z}) p(\tau \mid \mathcal{Y}, \mathbf{z}) .
$$

For the model in (2.58) the first factor is a Gaussian distribution, which can be obtained using, e.g., weighted least squares (4.9) as in [95], resulting in

$$
p(\mathcal{X} \mid \tau, \mathcal{Y}, \mathbf{z})=\mathcal{N}\left(\mathcal{X} \mid \hat{\mathcal{X}}^{\tau}, \mathbf{P}^{\tau}\right) \text {. }
$$

The posterior distribution of $\tau$ in (4.47) can be computed using Bayes' theorem and marginalization as

$$
w_{\tau} \triangleq p(\tau \mid \mathcal{Y}, \mathbf{z}) \propto p(\tau) p(\mathbf{z} \mid \tau, \mathcal{Y})=p(\tau) \int p\left(\mathbf{z} \mid \mathbf{x}_{\tau}, \tau\right) p\left(\mathbf{x}_{\tau} \mid \tau, \mathcal{Y}\right) d \mathbf{x}_{\tau} .
$$

In (4.49) the first factor in the integrand is given by $(2.58 \mathrm{c})$ and the second factor is the smoothed posterior distribution of the state given only measurements with precise timestamps, which for the model in (2.58) is a Gaussian distribution, $\mathcal{N}\left(\mathbf{x}_{\tau} \mid \hat{\mathbf{x}}_{\tau}^{y}, \mathbf{P}_{\tau}^{y}\right)$. The distribution can be computed efficiently using the RTSS [97] described in Section 4.3 or the Forward-Backward smoother [50]. Evaluating the integral in (4.49) results in

$$
\begin{aligned}
w_{\tau} & \propto p(\tau) \int \mathcal{N}\left(\mathbf{z} \mid \mathbf{H}^{z} \mathbf{x}_{\tau}, \mathbf{R}^{z}\right) \mathcal{N}\left(\mathbf{x}_{\tau} \mid \hat{\mathbf{x}}_{\tau}^{y}, \mathbf{P}_{\tau}^{y}\right) d \mathbf{x}_{\tau} \\
& =p(\tau) \mathcal{N}\left(\mathbf{z} \mid \mathbf{H}^{z} \hat{\mathbf{x}}_{\tau}^{y}, \mathbf{H}^{z} \mathbf{P}_{\tau}^{y}\left(\mathbf{H}^{z}\right)^{T}+\mathbf{R}^{z}\right)=p(\tau) \mathcal{N}\left(\mathbf{z} \mid \hat{\mathbf{z}}_{\tau}, \mathbf{S}_{\tau}\right),
\end{aligned}
$$

and combining (4.45), (4.47), (4.48) and (4.50) results in the Gaussian mixture

$$
p(\mathcal{X} \mid \mathcal{Y}, \mathbf{z})=\sum_{\tau \in \mathcal{K}} w_{\tau} \mathcal{N}\left(\mathcal{X} \mid \hat{\mathcal{X}}^{\tau}, \mathbf{P}^{\tau}\right)
$$

Similarly, the marginalization of the posterior distribution is derived as

$$
p\left(\mathbf{x}_{k} \mid \mathcal{Y}, \mathbf{z}\right)=\sum_{\tau \in \mathcal{K}} w_{\tau} \mathcal{N}\left(\mathbf{x}_{k} \mid \hat{\mathbf{x}}_{k}^{\tau}, \mathbf{P}_{k}^{\tau}\right),
$$

where $\mathcal{N}\left(\mathbf{x}_{k} \mid \hat{\mathbf{x}}_{k}^{\tau}, \mathbf{P}_{k}^{\tau}\right)$ is the smoothed posterior distribution of the state conditional on $\tau, \mathbf{z}$ and $\mathcal{Y}$.

The derivations in this section are used to compute the posterior distributions for the simple uncertain time scenario in Application 4.2. 


\section{Application 4.2: Simple Uncertain Time Posterior Distributions}

The posterior distributions derived in this section for a UTM are computed for the two cases of the simple scenario defined in Application 2.11.

The marginal posterior distribution $p\left(x_{k} \mid y_{1}, y_{N}, z\right)$ is computed using (4.52) and is shown in Figures 4.6 and 4.7 for the first and second case, respectively. The distribution $p\left(\tau \mid y_{1}, y_{N}, z\right)$ is computed using (4.50) and is shown, together with the prior distribution $p(\tau)$, in Figures 4.8 and 4.9 for the first and second case, respectively. In addition, $\max _{\mathcal{X}} p\left(\mathcal{X}, \tau \mid y_{1}, y_{N}, z\right)$ is also computed and displayed for comparison.

Assuming the underlying system to be continuous and the measurements and the observation to be noise free, the intermediate value theorem [1] for the first model already gives the information that $x(\tilde{\tau})=z$ for some continuous $\tilde{\tau}$, so at a first glance the observation does not seem to add any new information. However, since $\tau$ is random, the state is more likely to be in the vicinity of the observation, explaining the shape of the posterior distribution in Figure 4.6. For the second model in Figure 4.7 the prior distribution is more informative and the observation is also not one guaranteed by the intermediate value theorem, thus it provides more information than in the first case.

In Figure 4.9 the distributions do not differ much, but are slightly shifted to account for the observation being closer to the last measurement than the first measurement. Figure 4.8 is less intuitive. The unusual shape of $p\left(\tau \mid y_{1}, y_{N}, z\right)$ is further analysed in Application 4.7, where the distribution is compared to $\max _{\mathcal{X}} p\left(\mathcal{X}, \tau \mid y_{1}, y_{N}, z\right)$.

\subsubsection{Point Estimators}

In many situations one is interested in point estimates for the posterior distributions in Section 4.6.1, e.g., for presentation, where visualising the posterior distribution often is difficult. Point estimators are straightforward for Gaussian distributions [76], because they are symmetric and unimodal. The posterior distributions derived in Section 4.6.1 are Gaussian mixtures, that in general are neither symmetric nor unimodal, which means that obtaining point estimates is more difficult. Two common options are the minimum mean squared error (MMSE) estimator and the maximum a posteriori (MAP) estimator, which are derived for the model in (2.58). In addition, the joint maximum a posteriori (JMAP) estimator is also considered.

\section{Minimum Mean Squared Error Estimator}

The MMSE estimator, derived in [76], of the posterior distribution $p(\mathcal{X} \mid \mathcal{Y}, \mathbf{z})$ in (4.51) is given by

$$
\begin{aligned}
\hat{\mathcal{X}}^{\mathrm{MMSE}} & =\underset{\hat{\mathcal{X}}}{\arg \min } E_{\mathcal{X} \mid \mathcal{Y}, \mathbf{z}}\left[(\hat{\mathcal{X}}-\mathcal{X})^{T}(\hat{\mathcal{X}}-\mathcal{X})\right] \\
& =E_{\mathcal{X} \mid \mathcal{Y}, \mathbf{z}}[\mathcal{X}]=\sum_{\tau \in \mathcal{K}} w_{\tau} \hat{\mathcal{X}}^{\tau},
\end{aligned}
$$




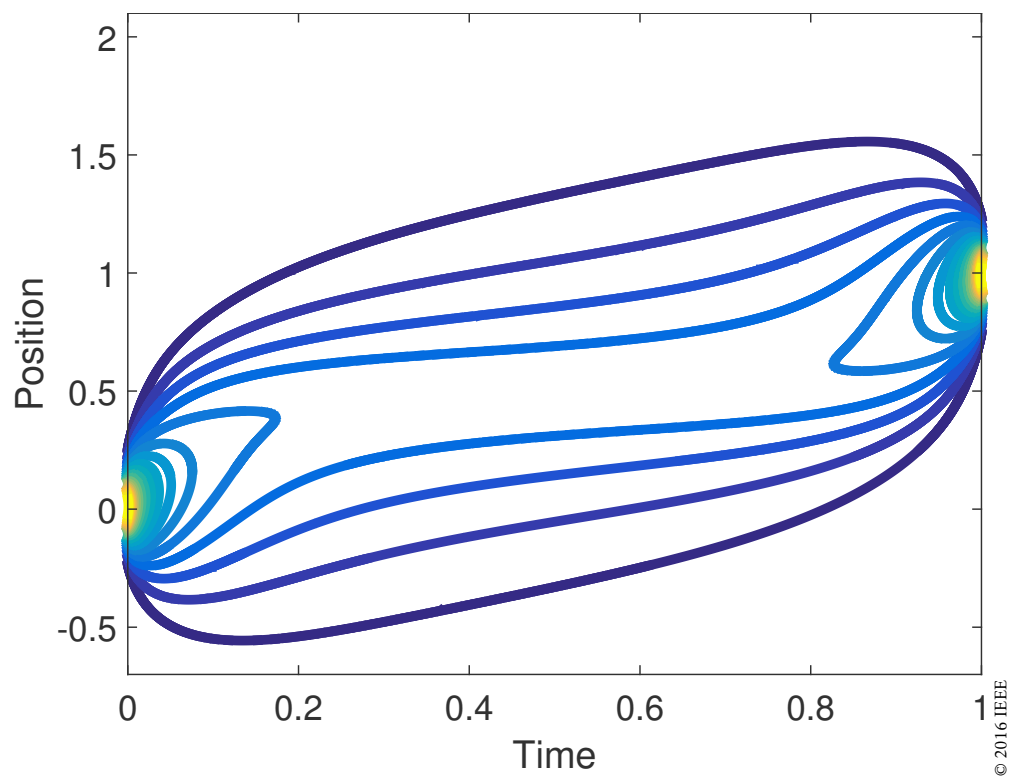

Figure 4.6: Contour plot of the posterior distribution for the first case. Published in [113].

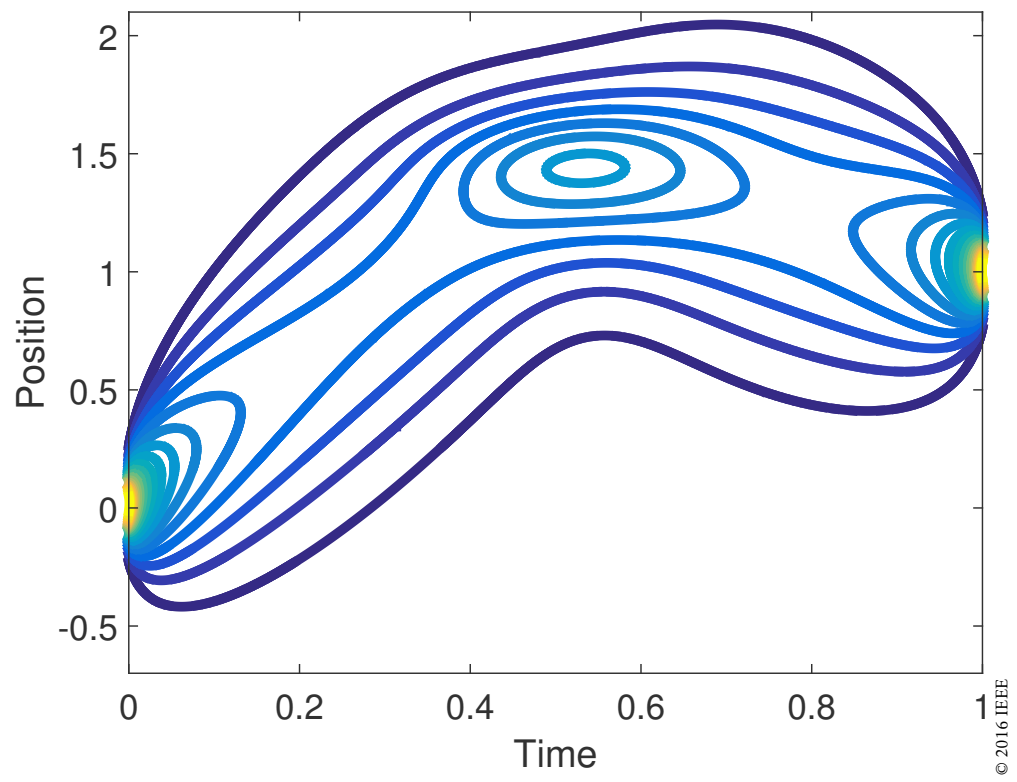

Figure 4.7: Contour plot of the posterior distribution for the second case. Published in [113]. 


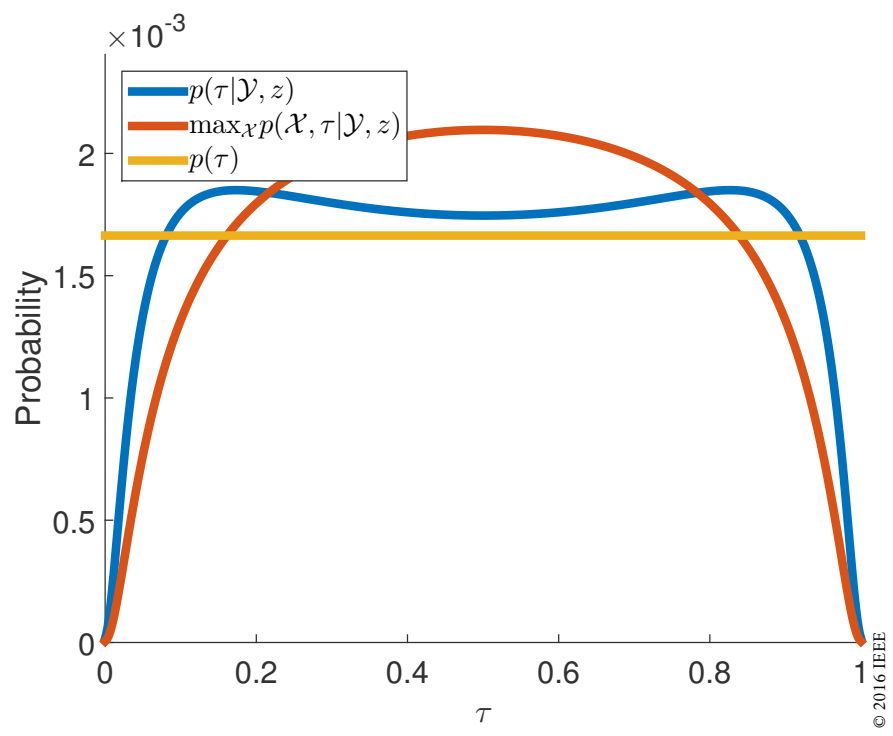

Figure 4.8: The distributions $p\left(\tau \mid y_{1}, y_{N}, z\right), \max _{\mathcal{X}} p\left(\mathcal{X}, \tau \mid y_{1}, y_{N}, z\right)$ and $p(\tau)$ for the first case. Published in [113].

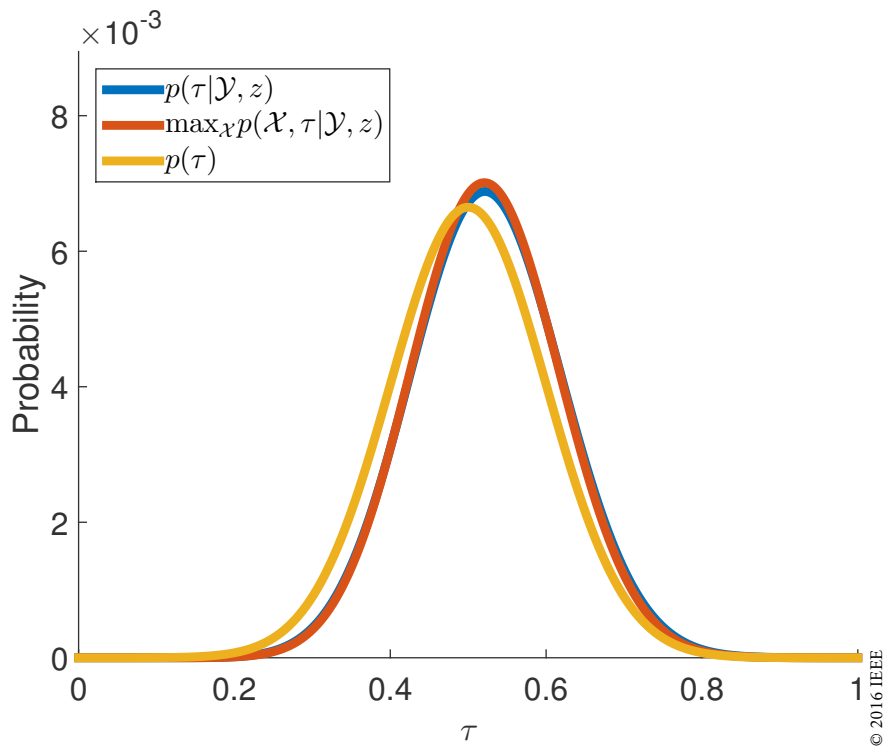

Figure 4.9: The distributions $p\left(\tau \mid y_{1}, y_{N}, z\right), \max _{\mathcal{X}} p\left(\mathcal{X}, \tau \mid y_{1}, y_{N}, z\right)$ and $p(\tau)$ for the second case. Published in [113]. 
which also minimizes the mean squared error (MSE) for each component, resulting in an identical MMSE estimator for the marginalized posterior distribution for each state, $p\left(\mathbf{x}_{k} \mid \mathcal{Y}, \mathbf{z}\right)$.

The classical MSE is

$$
\mathbf{P}^{\mathrm{MMSE}}=\sum_{\tau \in \mathcal{K}} w_{\tau}\left(\mathbf{P}^{\tau}+\left(\hat{\mathcal{X}}^{\tau}-\hat{\mathcal{X}}^{\mathrm{MMSE}}\right)\left(\hat{\mathcal{X}}^{\tau}-\hat{\mathcal{X}}^{\mathrm{MMSE}}\right)^{T}\right),
$$

which is an approximate estimate of the performance of the estimator. Note that this is the classical MSE, as described in [76], rather than the Bayesian MSE, which is more complicated to compute due to the additional expectation with respect to the measurements and observation.

The MMSE estimator for the states in the simple uncertain time scenario is presented in Application 4.3.

\section{Application 4.3: Simple Uncertain Time MMSE Estimator}

The MMSE estimate is shown in Figures 4.10 and 4.11 for the first and second case, respectively, together with the uncertainty and the estimate using only measurements with precise timestamps.

The MMSE estimator for the orienteering application is presented in Application 4.3. The resulting trajectory of the sprinter when using the proposed model in this application is also compared to the original trajectory using only the GNSS measurements.

\section{Application 4.4: Orienteering Uncertain Time MMSE Estimator}

The posterior distribution of the trajectory in the orienteering application is difficult to visualise in two dimensions, so as an alternative the MMSE estimate is used for a short analysis. The MMSE estimate for the orienteering example is shown in Figure 4.12. The bias has been estimated and the trajectory of the sprinter has been adjusted to pass through the control.

The improvement of the trajectory is limited for this particular set of data considering the GNSS measurement was rather accurate to begin with. However, the effects are present and it can be seen as a motivation for further development of the method. For more difficult terrain with large differences in altitude the GNSS is often less reliable. Further development of the method should also include the remaining control points for an improved estimate of the trajectory for the entire race. 


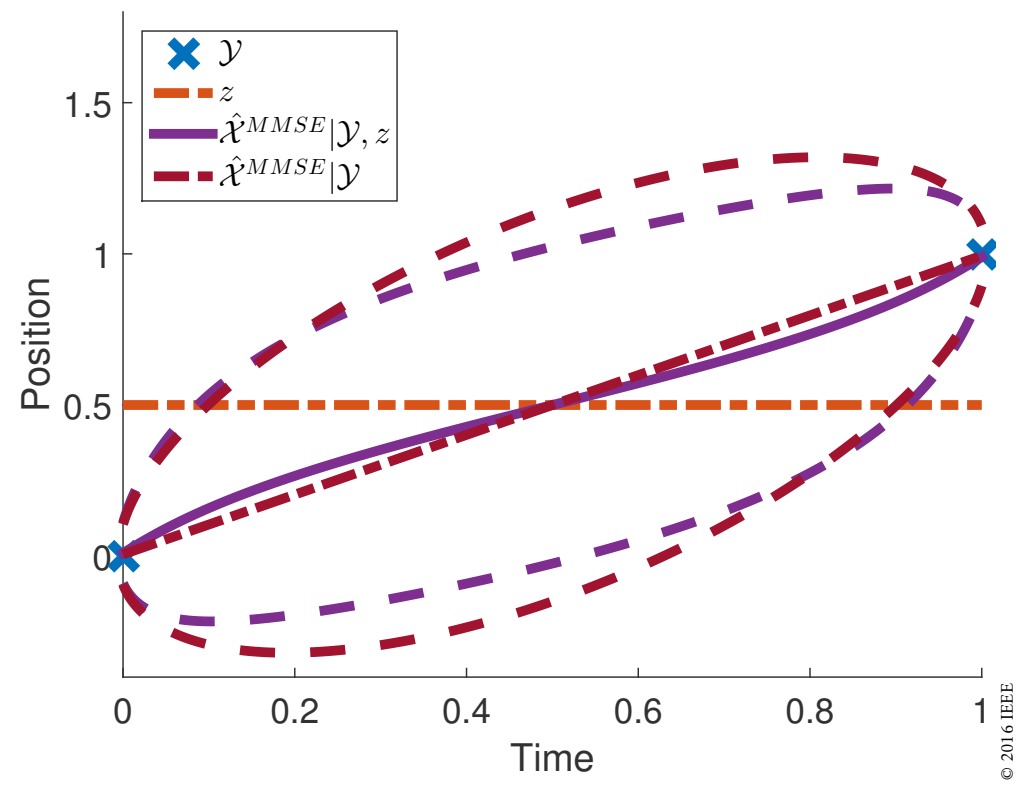

Figure 4.10: The MMSE estimate and its uncertainty for the first case compared to the MMSE estimate given only measurements. Published in [113].

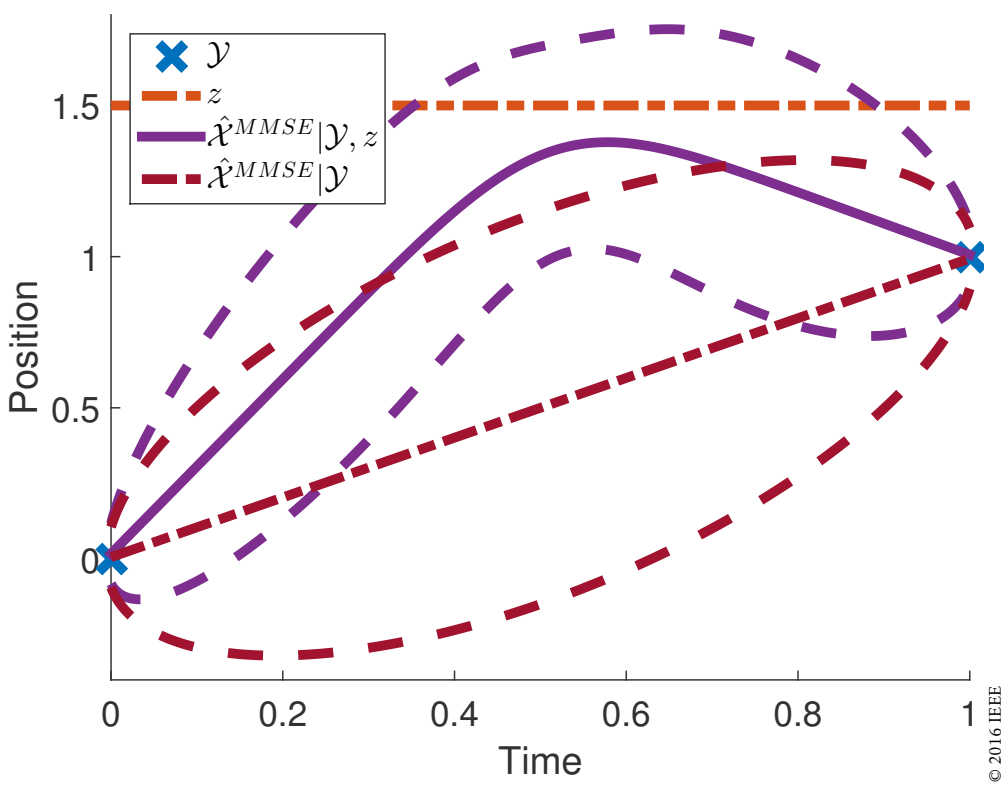

Figure 4.11: The MMSE estimate and its uncertainty for the second case compared to the MMSE estimate given only measurements. Published in [113]. 


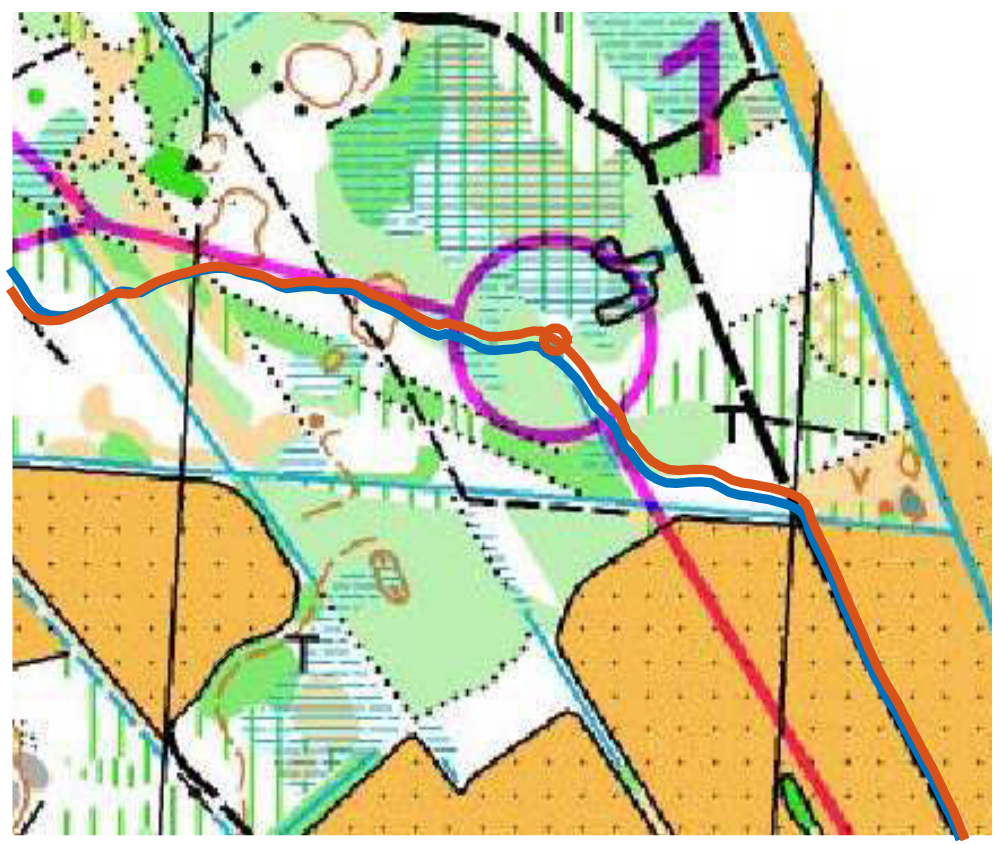

Figure 4.12: The MMSE estimate for the orienteering example compared to the GPS measurements. Courtesy of OK Orion.

\section{Maximum A Posteriori Estimator}

The MAP estimator, derived in [76], of the joint posterior distribution of all states, $p(\mathcal{X} \mid \mathcal{Y}, \mathbf{z})$, is given by

$$
\begin{aligned}
\hat{\mathcal{X}}^{\mathrm{MAP}} & =\underset{\mathcal{X}}{\arg \max } p(\mathcal{X} \mid \mathcal{Y}, \mathbf{z}) \\
& =\underset{\mathcal{X}}{\arg \max } \sum_{\tau \in \mathcal{K}} p(\mathcal{X}, \tau \mid \mathcal{Y}, \mathbf{z}),
\end{aligned}
$$

where $p(\mathcal{X}, \tau \mid \mathcal{Y}, \mathbf{z})$ is preferably computed using (4.46) to avoid computing the smoothed joint posterior distribution of all states conditional on all $\tau$. Even for the model in Section 2.7 it is often difficult to maximize (4.55) considering the high dimensionality and that Gaussian mixtures in general have multiple local maxima.

An approximation is to assume independence between the states and compute the MAP estimate of $p\left(\mathbf{x}_{k} \mid \mathcal{Y}, \mathbf{z}\right)$ in (4.52) for each state separately,

$$
\begin{aligned}
\hat{\mathbf{x}}_{k}^{\mathrm{MAP}} & =\underset{\mathbf{x}_{k}}{\arg \max } p\left(\mathbf{x}_{k} \mid \mathcal{Y}, \mathbf{z}\right) \\
& =\underset{\mathbf{x}_{k}}{\arg \max } \sum_{\tau \in \mathcal{K}} w_{\tau} \mathcal{N}\left(\mathbf{x}_{k} \mid \hat{\mathbf{x}}_{k}^{\tau}, \mathbf{P}_{k}^{\tau}\right), \quad k \in \mathcal{K} .
\end{aligned}
$$


Maximization of a Gaussian mixture is still required, but the dimensionality is significantly reduced.

To estimate the uncertain times, the MAP estimate of (4.50) can be computed as

$$
\begin{aligned}
\hat{\tau}^{\mathrm{MAP}} & =\underset{\tau}{\arg \max }\left\{p(\tau) \mathcal{N}\left(\mathbf{z} \mid \hat{\mathbf{z}}_{\tau}, \mathbf{S}_{\tau}\right)\right\} \\
& =\underset{\tau}{\arg \max }\left\{2 \ln p(\tau)-\left\|\mathbf{z}-\hat{\mathbf{z}}_{\tau}\right\|_{\mathbf{S}_{\tau}}^{2}-\ln \left|\mathbf{S}_{\tau}\right|\right\},
\end{aligned}
$$

where the monotonically increasing property of logarithms is used in the second equality.

The MAP estimator for the simple uncertain time scenario is presented in Application 4.5.

\section{Application 4.5: Simple Uncertain Time MAP Estimator}

The MAP estimates of $p\left(\mathcal{X} \mid y_{1}, y_{N}, z\right)$ and $p\left(x_{k} \mid y_{1}, y_{N}, z\right)$ are presented in Figures 4.13 and 4.14 together with the credible interval for $p\left(x_{k} \mid y_{1}, y_{N}, z\right)$. The estimates are computed with the quasi-Newton numerical optimization method [39] on (4.45), using (4.46), and (4.52), respectively, without a guarantee of convergence to the global maximum. The MMSE estimator using only measurements with precise timestamps, which is equal to the MAP estimator for this case, is also shown for comparison.

\section{Joint Maximum A Posteriori Estimate}

The JMAP estimator of the joint posterior distribution $p(\mathcal{X}, \tau \mid \mathcal{Y}, \mathbf{z})$ is given by

$$
\begin{aligned}
\left\{\hat{\mathcal{X}}^{\mathrm{JMAP}}, \hat{\tau}^{\mathrm{JMAP}}\right\} & =\underset{\mathcal{X}, \tau}{\arg \max } p(\mathcal{X}, \tau \mid \mathcal{Y}, \mathbf{z}) \\
& =\underset{\mathcal{X}, \tau}{\arg \max } p(\mathcal{X} \mid \tau, \mathcal{Y}, \mathbf{z}) p(\tau \mid \mathcal{Y}, \mathbf{z}) \\
& =\underset{\mathcal{X}, \tau}{\arg \max } w_{\tau} \mathcal{N}\left(\mathcal{X} \mid \hat{\mathcal{X}}^{\tau}, \mathbf{P}^{\tau}\right),
\end{aligned}
$$

where (4.47)-(4.49) are used, which further reduces to

$$
\begin{aligned}
\hat{\tau}^{\mathrm{MMAP}} & =\underset{\tau}{\arg \max }\left\{\max _{\mathcal{X}} w_{\tau} \mathcal{N}\left(\mathcal{X} \mid \hat{\mathcal{X}}^{\tau}, \mathbf{P}^{\tau}\right)\right\}=\underset{\tau}{\arg \max } \frac{w_{\tau}}{\sqrt{\left|\mathbf{P}^{\tau}\right|}} \\
& =\underset{\tau}{\arg \max }\left\{w_{\tau} \sqrt{\left|\mathbf{R}^{z}+\mathbf{H}^{z} \mathbf{P}_{\tau}\left(\mathbf{H}^{z}\right)^{T}\right|}\right\} \\
& =\underset{\tau}{\arg \max }\left\{p(\tau) \mathcal{N}\left(\mathbf{z} \mid \hat{\mathbf{z}}_{\tau}, \mathbf{S}_{\tau}\right) \sqrt{\left|\mathbf{S}_{\tau}\right|}\right\} \\
& =\underset{\tau}{\arg \max }\left\{2 \ln p(\tau)-\left\|\mathbf{z}-\hat{\mathbf{z}}_{\tau}\right\|_{\mathbf{S}_{\tau}}^{2}\right\}, \\
\hat{\mathcal{X}}^{\mathrm{JMAP}} & =\hat{\mathcal{X}}^{\hat{\tau}^{\mathrm{JMAP}}},
\end{aligned}
$$

where Proposition 4.2 and (4.50) are used. Note that the estimates do not depend on the joint covariance of all states, only the covariance of each state. 


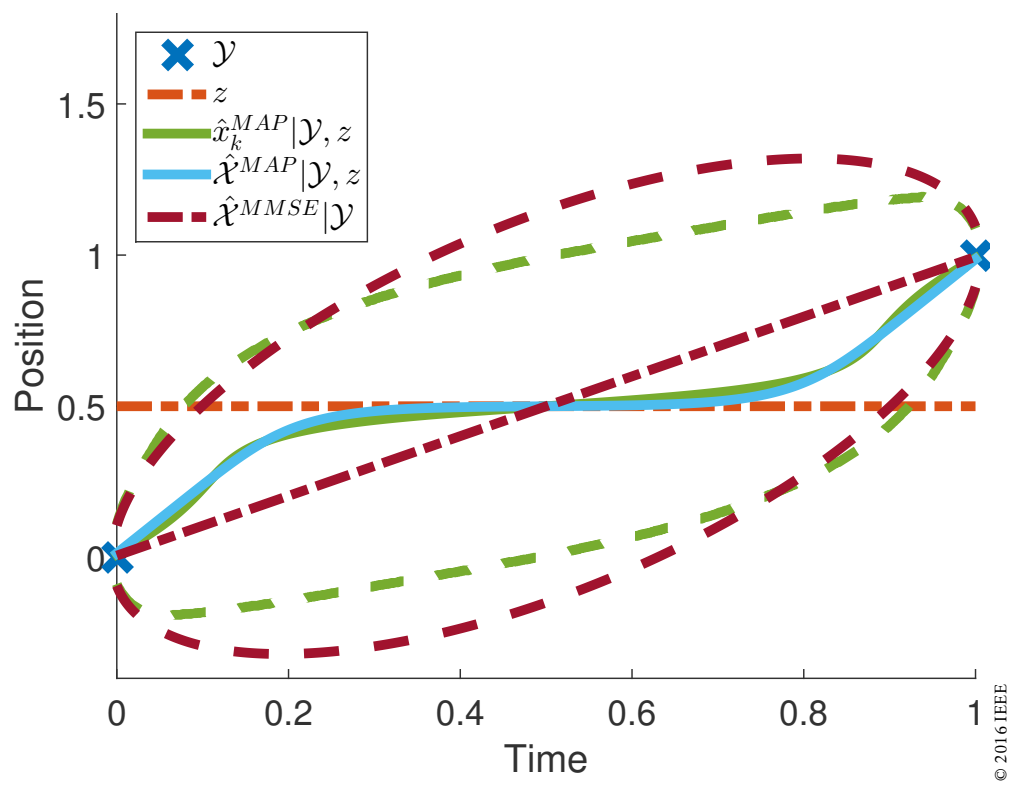

Figure 4.13: The MAP estimates of $p\left(\mathcal{X} \mid y_{1}, y_{N}, z\right)$ and $p\left(x_{k} \mid y_{1}, y_{N}, z\right)$ for the first case and the MMSE estimate given only the measurements. Published in [113].

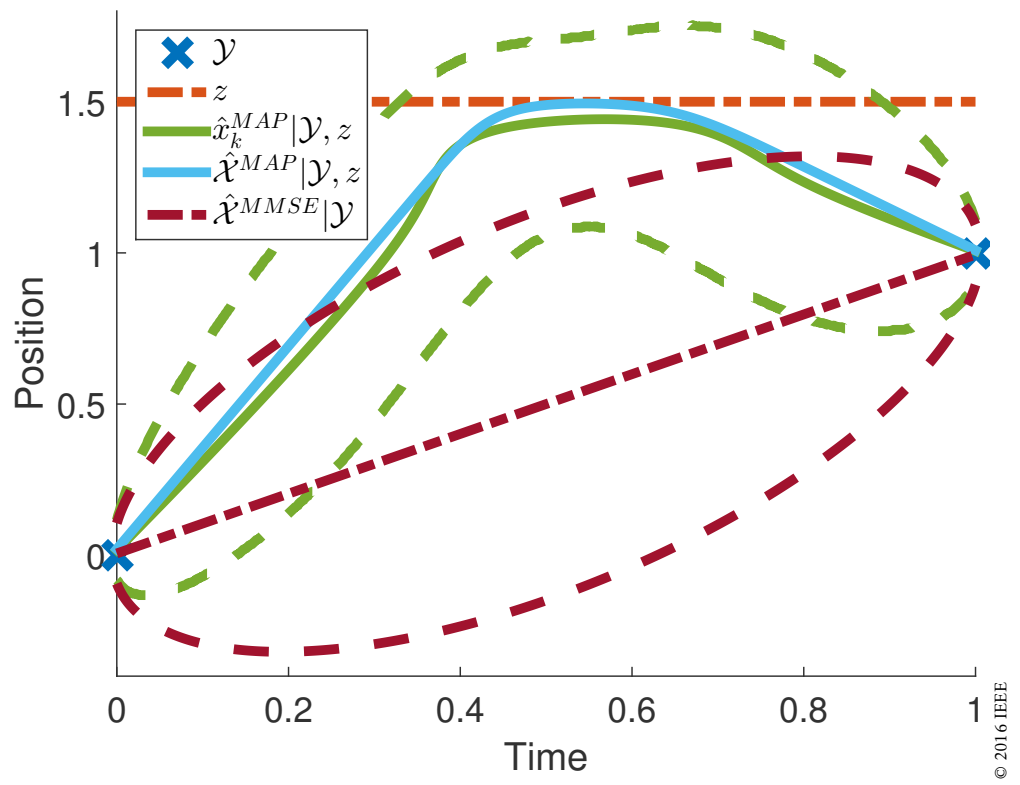

Figure 4.14: The MAP estimates of $p\left(\mathcal{X} \mid y_{1}, y_{N}, z\right)$ and $p\left(x_{k} \mid y_{1}, y_{N}, z\right)$ for the second case and the MMSE estimate given only the measurements. Published in [113]. 
Proposition 4.2. Given a joint posterior distribution $p(\mathcal{X} \mid \mathcal{Y})$ of a UTM described by (2.58), where $\mathbf{P}=\operatorname{Cov}(\mathcal{X} \mid \mathcal{Y}), \mathbf{P}_{k}=\operatorname{Cov}\left(\mathbf{x}_{k} \mid \mathcal{Y}\right)$ and $\mathbf{P}$ and $\mathbf{R}$ are non-singular, the determinant of $\mathbf{P}^{\tau}=\operatorname{Cov}(\mathcal{X} \mid \mathcal{Y}, \mathbf{z}, \tau)$ is given by

$$
\left|\mathbf{P}^{\tau}\right|=|\mathbf{P}||\mathbf{R}|\left|\mathbf{R}+\mathbf{H}^{z} \mathbf{P}_{\tau} \mathbf{H}^{T}\right|^{-1} \text {. }
$$

Proof: Let the observation be $\mathbf{z}=\mathbf{H} \mathbf{x}_{\tau}+\mathbf{v}=\overline{\mathbf{H}}_{\tau} \mathcal{X}+\mathbf{v}$, then

$$
\begin{aligned}
\left|\mathbf{P}^{\tau}\right|^{-1} & =\left|\left(\mathbf{P}^{-1}+\overline{\mathbf{H}}_{\tau}^{T} \mathbf{R}^{-1} \overline{\mathbf{H}}_{\tau}\right)^{-1}\right|^{-1} \\
& =\left|\mathbf{P}^{-1}+\overline{\mathbf{H}}_{\tau}^{T} \mathbf{R}^{-1} \overline{\mathbf{H}}_{\tau}\right| \\
& =|\mathbf{P}|^{-1}\left|\mathbf{I}+\mathbf{R}^{-1} \overline{\mathbf{H}}_{\tau} \mathbf{P} \overline{\mathbf{H}}_{\tau}^{T}\right| \\
& =|\mathbf{P}|^{-1}|\mathbf{R}|^{-1}\left|\mathbf{R}+\mathbf{H} \mathbf{P}_{\tau} \mathbf{H}^{T}\right|,
\end{aligned}
$$

where Millman's formula [2] is used in the first equality and Sylvester's identity [3] in the third equality, concluding the proof.

The JMAP estimator for the simple uncertain time scenario is presented in Application 4.3 .

Application 4.6: Simple Uncertain Time JMAP Estimator

The JMAP state estimate $\hat{\mathcal{X}}^{\text {JMAP }}$ is shown in Figures 4.15 and 4.16 for the first and second case, respectively, together with the uncertainty of the state estimate given that the JMAP time estimate is correct. Since the uncertainty in time is disregarded, the uncertainty for the state estimate is underestimated. The estimated uncertain times $\hat{\tau}^{\mathrm{JMAP}}$ are 0.5 and 0.52 , respectively.

\section{Comparison of Point Estimators}

An advantage of the MMSE estimator is that it is unbiased, however for multimodal distributions a disadvantage is that the estimate might fall between modes, producing an unlikely estimate. The MAP and JMAP estimators will on the other hand produce likely estimates, but at the cost of being biased.

Often, the reason for using the MAP estimator over the MMSE estimator is that it is cheaper to compute since it avoids an expensive integration over the states. This is not the case for the Gaussian distribution, as well as for Gaussian mixtures, where this integration is analytical. Both the MAP and MMSE estimators, on the other hand, require a summation over all possible $\tau$, for which the complexity increases exponentially with the number of observations with uncertain timestamps. However, this is not a problem for the JMAP estimator, which lends itself more easily to be computed using efficient methods, e.g., stochastic methods [42] or the EM algorithm [83].

Another disadvantage of the MAP estimator is that it requires numerical optimization methods for Gaussian mixtures. The dimension increases with decreased sampling time $T$, which commonly is chosen small for good resolution, and already the simple model considered in Application 4.2 is difficult. As an alternative, the posterior distribution of each state can be maximized, resulting in 


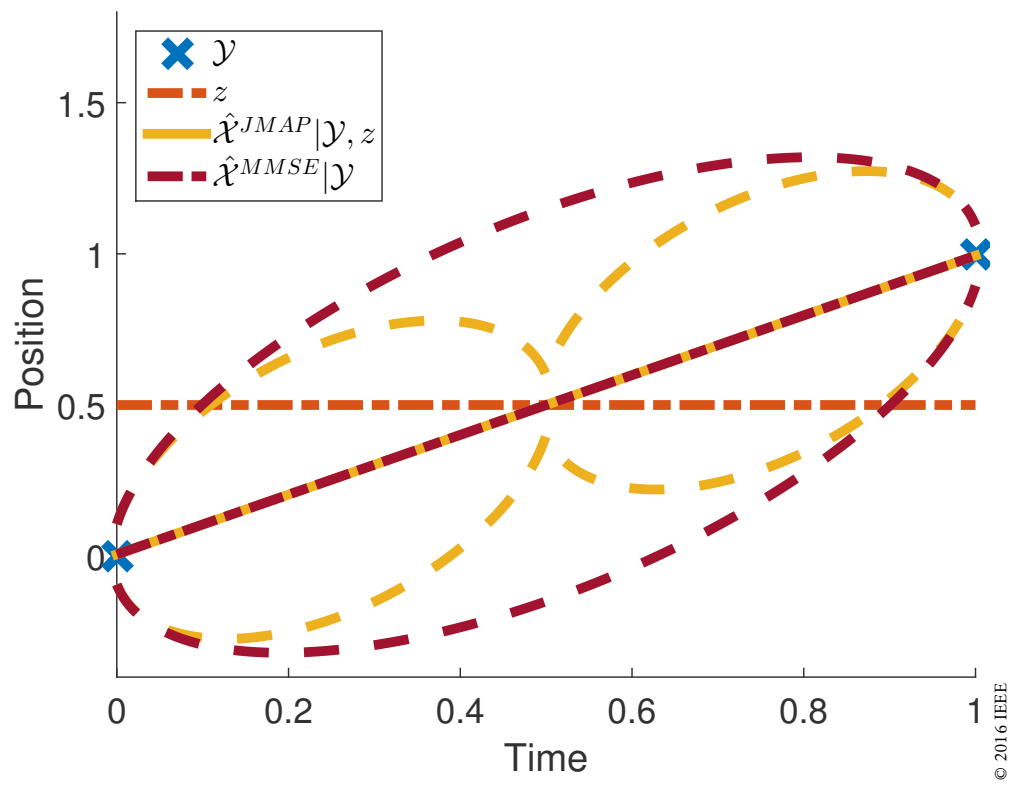

Figure 4.15: JMAP state estimate of $p\left(\mathcal{X}, \tau \mid y_{1}, y_{N}, z\right)$ for the first case and the MMSE estimate given only the measurements. Published in [113].

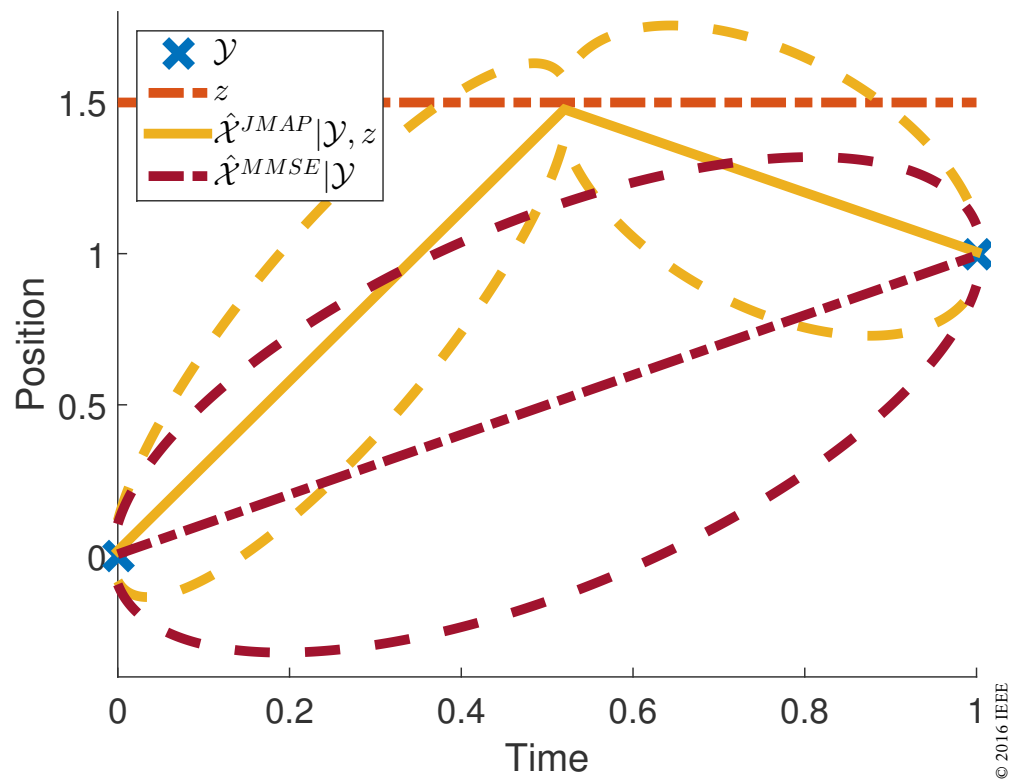

Figure 4.16: JMAP state estimate of $p\left(\mathcal{X}, \tau \mid y_{1}, y_{N}, z\right)$ for the second case and the MMSE estimate given only the measurements. Published in [113]. 
multiple low-dimensional optimization problems, but as is argued in [76] the estimators do not produce the same result. The reason for this is that the crosscovariances are ignored. Another issue is that Gaussian mixtures possibly are multimodal and it might be difficult to find optimization methods that guarantee finding the global optimum.

A comparison and an analysis of the various estimators for the simple uncertain time scenario are presented in Application 4.7.

\section{Application 4.7: Simple Uncertain Time Estimator Comparison}

The effects of the additional observation sampled at an uncertain time on the estimators are analysed. The analysis is based on the results presented in Applications 4.3, 4.5 and 4.6. Further, the posterior distribution of the timestamp presented in Application 4.2 is shortly discussed.

The effects of including an observation with an uncertain timestamp is most visible for the first case. Since the observation coincides with the previous trajectory, i.e., the estimate given only the measurements with precise timestamps, the JMAP estimator in Figure 4.15 will estimate $\tau$ at the time of coincidence and result in the same trajectory. The uncertainty is underestimated because it disregards the uncertainty in time.

The MMSE estimator in Figure 4.10 produces a trajectory that slightly tends towards the observation compared to the previous trajectory. The reason for this behaviour can be found in Figure 4.8 where most weights, given by $p\left(\tau \mid y_{1}, y_{N}, z\right)$, are similar except near the measurements with precise timestamps. The trajectory for each weight passes near the observation at its corresponding time, attracting the weighted average of the trajectories in the MMSE estimate towards it. It should also be noted that the uncertainty for the estimate is reduced compared to the previous trajectory.

The behaviour of tending towards the observation is even more pronounced for the MAP estimators in Figure 4.13. For the MAP estimator of $p\left(x_{k} \mid y_{1}, y_{N}, z\right)$, this can intuitively be understood by realizing that at each time within the region of similar weights in Figure 4.8 the heights of the peaks will be approximately proportional to the inverse of the covariance, and the smallest covariance is given by the Gaussian where the observation was used to update the posterior distribution at the current time. This will attract the trajectory even more towards the observation than the MMSE estimate. Although harder to visualise, due to the high dimension, a similar intuition applies to the MAP estimator for $p\left(\mathcal{X} \mid y_{1}, y_{N}, z\right)$. The MAP estimate of $p\left(\mathcal{X} \mid y_{1}, y_{N}, z\right)$ is also smoother than the MAP estimate of $p\left(x_{k} \mid y_{1}, y_{N}, z\right)$, which can be explained by the discarded cross-covariances in the latter. The uncertainty of $p\left(x_{k} \mid y_{1}, y_{N}, z\right)$ is also reduced significantly over the previous trajectory.

For the second case the estimates are drastically different since the observation does not coincide with the previous trajectory. The other difference compared to the first case is that the prior distribution $p(\tilde{\tau})$ is no longer flat, see Figure 4.9 . 
This gives much more influence to trajectories corresponding to the peaked region. The JMAP estimator in Figure 4.16 produces the trajectory that corresponds to the maximum of $p\left(\mathcal{X}, \tau \mid y_{1}, y_{N}, z\right)$. As before, the MMSE estimate in Figure 4.11 is a weighted average of trajectories, thus not reaching all the way to the observation. The MAP estimates $p\left(x_{k} \mid y_{1}, y_{N}, z\right)$ and $p\left(\mathcal{X} \mid y_{1}, y_{N}, z\right)$ tend even more to the observation, which can be explained with similar intuition as for the first case.

The derivations for the MAP and JMAP estimators allows for further analysis of the posterior distribution of the timestamp in Figure 4.8. As can be seen in (4.59a) $\max _{\mathcal{X}} p\left(\mathcal{X}, \tau \mid y_{1}, y_{N}, z\right)$ depends on the prior distribution, which is constant in this case, as well as the Mahalanobis distance between the observation and its prediction. In (4.57), however, it can be seen that $p\left(\tau \mid y_{1}, y_{N}, z\right)$ in addition depends on the determinant of the innovation covariance, in effect penalizing uncertainty in the likelihood of $z$. By considering $\max _{\mathcal{X}} p\left(\mathcal{X}, \tau \mid y_{1}, y_{N}, z\right)$, the reduction of uncertainty in the joint posterior distribution is also taken into account, while only the likelihood of the observation given the measurements is considered in $p\left(\tau \mid y_{1}, y_{N}, z\right)$.

For further analysis, the impact of multiple observations sampled at uncertain times needs to be considered. As mentioned previously, such a scenario results in an exponentially increasing computational cost. Stochastic methods would be suitable for approximating the posterior distributions and estimators. 


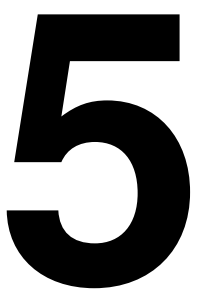

\section{Data Association}

Data association in the context of target tracking is the problem of associating measurements to existing tracks when there is an uncertainty in association. Association uncertainty arises when multiple targets are present in the scene or when false detections occur. There are several established methods available to handle the uncertainty. They vary in complexity and options for improvement, allowing the designer to select a suitable approach for meeting the requirements of a particular application. There is an abundance of literature covering the topic of data association and target tracking, especially with a focus on military applications. Common principles and an overview of established methods are described in, e.g., [11] and [25]. For more details on implementation in particular applications, see e.g. [12], [9] and [14]. More modern methods are described in, e.g., [86] and [107].

The brute-force approach is to consider all possible associations between measurements and targets, as well as the possibility of new targets and false detections, and select the most probable hypothesis. The combinatorial complexity of this approach is intractable to handle, so methods strive to reduce this complexity without degrading performance more than necessary.

An outline of data association uncertainty and how it relates to target tracking is given in Section 1.2. Some concepts related to data association uncertainty are described in Section 5.1. Two target tracking algorithms, the MHT and the PDA filter, for solving the data association problem are presented in Sections 5.2 and 5.3, respectively. A modification of the PDA filter, previously described by the author in [112], is presented in Section 5.3.3. 


\subsection{Data Association Uncertainty}

Given at each time step is a set of existing tracks and a set of measurements, and the task is to associate the measurements to existing tracks, new tracks or identify as false detections. The relevant elements are modelled probabilistically to allow for a comparison between different association hypotheses. These concepts and a formulation of the assignment problem are briefly described in this section. More details on the subject are given in, e.g., [25].

\subsubsection{Uncertainty Models}

In this section, a few common models for the uncertainty of false detections, new targets and missed detections are described briefly. Some standard models are outlined for these concepts that, with some variations, will be used subsequently in the chapter. For a more detailed description, see e.g. [25].

At the current time step each target in the field of view generates a single detection with probability $P_{D}$. This results in a set $\mathcal{Y}_{e}=\left\{\mathbf{y}_{i}^{e}\right\}_{i=1}^{M_{e}}$ of measurements for existing targets. False detections, or clutter, are traditionally modelled as Poisson and uniform distributed. The number $M_{f}$ of false detections is modelled with a Poisson distribution as

$$
p\left(M_{f}\right)=\mathcal{P} \mathcal{S}\left(M_{f} \mid V \beta_{f}\right)=\frac{\left(V \beta_{f}\right)^{M_{f}} e^{-V \beta_{f}}}{M_{f} !},
$$

where $\beta_{f}$ is the false detection density and $V$ is the size of the surveillance volume $\mathcal{V}$. This results in a set $\mathcal{Y}_{f}=\left\{\mathbf{y}_{i}^{f}\right\}_{i=1}^{M_{f}}$ of false detections. Each detection is independently and uniformly distributed over the surveillance volume $\mathcal{V}$ as

$$
p\left(\mathbf{y}_{i}^{f}\right)=\mathcal{U}\left(\mathbf{y}_{i}^{f} \mid \mathcal{V}\right)= \begin{cases}\frac{1}{V}, & \text { if } \mathbf{y}_{i}^{f} \in \mathcal{V}, \\ 0 . & \text { if } \mathbf{y}_{i}^{f} \notin \mathcal{V},\end{cases}
$$

New targets are similarly modelled as $\mathcal{Y}_{n}=\left\{\mathbf{y}_{i}^{n}\right\}_{i=1}^{M_{n}}$ with the new target density $\beta_{n}$ over the surveillance volume $\mathcal{V}$. The union of all detections is reindexed to discard the origins and delivered at time step $k$ as $\mathcal{Y}_{k}=\mathcal{Y}_{e} \cup \mathcal{Y}_{f} \cup \mathcal{Y}_{n}=\left\{\mathbf{y}_{k}^{i}\right\}_{i=1}^{M_{k}}$.

To handle the uncertainty in the origin inherent in the set of measurements $\mathcal{Y}_{k}$, hypotheses regarding the origin of each measurement are constructed. A hypothesis $\theta_{1: k}$ is a set of associations for all measurements $\mathcal{Y}_{1: k}=\left\{\mathcal{Y}_{i}\right\}_{i=1}^{k}$. A measurement is either associated to a target or identified as a false detection. All measurements associated to the same target in a hypothesis are used to estimate a track using a model. If no previous association to a target exists a new track is initialised. Targets with existing tracks are updated with the new measurements. A set of tracks $\mathcal{T}_{k}$ is estimated given the measurements $\mathcal{Y}_{1: k}$, a hypothesis $\theta_{1: k}$ and a model.

A track $\tau \in \mathcal{T}_{k}$ is estimated using the measurements $\mathcal{Y}_{1: k}^{\tau}$ associated to the track according to a hypothesis. The posterior distribution for the current state of the track is given by $p\left(\mathbf{x}_{k} \mid \tau\right)=p\left(\mathbf{x}_{k} \mid \mathcal{Y}_{1: k}^{\tau}\right)$. For the LGSSM the predicted measurement 
for a track $\tau \in \mathcal{T}_{k-1}$ is given by

$$
p\left(\mathbf{y}_{k} \mid \tau\right)=\mathcal{N}\left(\mathbf{y}_{k} \mid \hat{\mathbf{y}}_{k}^{\tau}, \mathbf{S}_{k}^{\tau}\right)
$$

where $\hat{\mathbf{y}}_{k}^{\tau}$ and $\mathbf{S}_{k}^{\tau}$ are computed using the KF in Algorithm 4.1 for the measurements used to form track $\tau$. An approximation of the prediction distribution for an NSSM can be obtained using the EKF.

To reduce the complexity or as a necessary part of the method, the measurements are often gated [25]. Gating essentially means that only measurements that could reasonably be generated by a tracked target are considered for association. Often a hierarchy of gates are used, with simple coarse gates at higher levels and a finer elliptical gate at the lowest level. Elliptical gates reject measurements from consideration if the statistical distance,

$$
d_{\tau}^{2}\left(\mathbf{y}_{j}\right)=\left(\mathbf{y}_{j}-\hat{\mathbf{y}}_{j}^{\tau}\right)^{T}\left(\mathbf{S}_{j}^{\tau}\right)^{-1}\left(\mathbf{y}_{j}-\hat{\mathbf{y}}_{j}^{\tau}\right) \sim \chi^{2}(m), \quad \tau \in \mathcal{T}_{j-1},
$$

is greater than a threshold $\gamma$, where $m$ is the dimension of $\mathbf{y}_{j}$. A fixed probability $P_{G}$ for the distribution can be used to select $\gamma$ or the threshold can be derived from the false detection and new track models [25].

\subsubsection{Track Score}

As measurements are associated to a track, a score is maintained for the track over time [25]. The track score function $L_{k}^{\tau}$ for a track $\tau \in \mathcal{T}_{k}$ is the logarithm of the likelihood ratio between the hypotheses $\theta_{T}^{\tau}$ and $\theta_{F}^{\tau}$ of the track $\tau$ being a true target and a false target, respectively, [25]

$$
L_{k}^{\tau}=\log \left(\frac{p\left(\theta_{T}^{\tau} \mid \tau\right)}{p\left(\theta_{F}^{\tau} \mid \tau\right)}\right) .
$$

The track score can be computed recursively, as derived in [15], where a new track is initialised as

$$
L_{1}^{\tau}=\log \left(\beta_{n}\right)-\log \left(\beta_{f}\right) .
$$

Let $D_{k}^{\tau}$ denote that a measurement, $\mathbf{y}_{k}^{\tau}$, is associated to a track $\tau \in \mathcal{T}_{k-1}$ at time step $k$ in a hypothesis. The track score for a track $\tau \in \mathcal{T}_{k-1}$ is then updated as

$$
L_{k}^{\tau}=L_{k-1}^{\tau}+ \begin{cases}\log \left(p\left(\mathbf{y}_{k}^{\tau} \mid \tau, \theta_{T}^{\tau}\right)\right)+\log \left(P_{D}\right)-\log \left(\beta_{f}\right), & \text { if } D_{k}^{\tau}, \\ \log \left(1-P_{D} P_{G}\right), & \text { if } \neg D_{k}^{\tau},\end{cases}
$$

where

$$
\begin{aligned}
\log \left(p\left(\mathbf{y}_{k}^{\tau} \mid \tau, \theta_{T}^{\tau}\right)\right) & =\log \mathcal{N}\left(\mathbf{y}_{k}^{\tau} \mid \hat{\mathbf{y}}_{k}^{\tau}, \mathbf{S}_{k}^{\tau}\right) \\
& =-\frac{1}{2} \log \left(\left|2 \pi \mathbf{S}_{k}^{\tau}\right|\right)-\frac{1}{2}\left(\mathbf{y}_{k}^{\tau}-\hat{\mathbf{y}}_{k}^{\tau}\right)^{T}\left(\mathbf{S}_{k}^{\tau}\right)^{-1}\left(\mathbf{y}_{k}^{\tau}-\hat{\mathbf{y}}_{k}^{\tau}\right),
\end{aligned}
$$

for an LGSSM. 
The probability of a hypothesis is computed using the recursion [15]

$$
p\left(\theta_{1: k} \mid \mathcal{Y}_{1: k}\right) \propto\left(\frac{\beta_{n}}{\beta_{f}}\right)^{M_{k}^{n}} \prod_{\tau \in \mathcal{T}_{k}^{D}} \frac{p\left(\mathbf{y}_{k}^{\tau} \mid \tau\right) P_{D}}{\beta_{f}} \cdot\left(1-P_{D} P_{G}\right)^{\left|\mathcal{T} \backslash \mathcal{I}_{D}\right|} \cdot p\left(\theta^{1: k-1} \mid \mathcal{Y}^{k-1}\right),
$$

where $\mathcal{T}_{k}^{D}=\left\{\tau \in \mathcal{T}_{k-1} \mid D_{k}^{\tau}\right\}$ is the set of all detected tracks, $M_{k}^{n}$ is the number of measurements associated with new tracks, $\left|\mathcal{T} \backslash \mathcal{T}_{D}\right|$ are the number of nondetected tracks and $p\left(\mathbf{y}_{k}^{\tau} \mid \tau\right)$ is the prediction distribution of track $\tau$ given in (5.3), according to the hypothesis $\theta_{1: k}$.

\subsubsection{Track Quality}

Given a track it is important to determine its quality. Before a target has generated a sufficient number of detections it is uncertain whether the track is a true target or a false detection. At a later stage, a target might disappear from the field of view, resulting in a sequence of missed detections and at some point the track needs to be declared dead.

A sequential probability ratio test (SPRT) [117] is performed to determine whether a track is a true target or a false target and if sufficient evidence has been received. Essentially, an upper threshold $\gamma_{h}$ and a lower threshold $\gamma_{l}$ are applied to the track score. Exceeding the upper threshold results in a confirmation of the track as a true target and falling below the lower threshold indicates a false target, resulting in the deletion of the track. The limits are computed as [25]

$$
\gamma_{h}=L_{1}+\log \left(\frac{1-P_{F D}}{P_{F C}}\right) \text { and } \gamma_{l}=L_{1}+\log \left(\frac{P_{F D}}{1-P_{F C}}\right) \text {, }
$$

where $P_{F D}$ is the accepted probability of falsely deleting a track for a true target and $P_{F C}$ is the accepted probability of confirming a track for a false target. The thresholds are set relative to the initial track score. Initially, before sufficient evidence is available to confirm a track, the track is commonly denoted an initiator or a tentative track.

A high quality track results in a high track score over time, making it slightly more difficult to set up a threshold for detecting a dead confirmed track. A common approach is to apply a threshold $\gamma_{d}$ relative to the maximum track score as

$$
\gamma_{d}=L_{k_{m}}+\log \left(P_{F D C}\right)
$$

where $k_{m}=\arg \max _{k} L_{k}$ and $P_{F D C}$ is the probability of falsely deleting an alive confirmed track.

An alternative method to using track scores is to instead count the number of detections and missed detections of a track and set up heuristic rules for confirmation and deletion, commonly denoted $M / N$-logic [24]. For example, a track might be confirmed if it is detected $N_{1}$ times in a row and subsequently $N_{2}$ out of $M$ times, otherwise deleted. Similarly, a confirmed track is deleted after $N_{D}$ missed detections. 


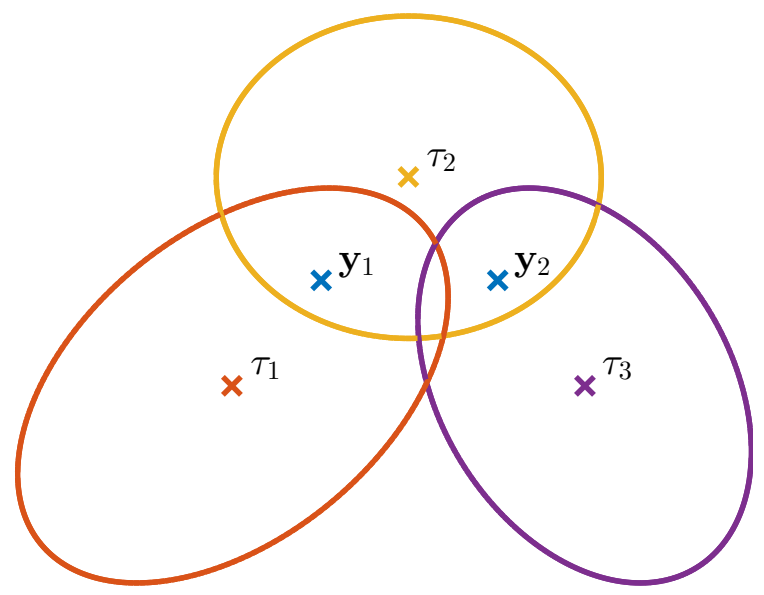

Figure 5.1: A simple scenario of three tracks with gates and two measurements.

The two methods perform similarly and approximate conversions between thresholds and rules can be obtained. However, due to the model-based nature of SPRT, this approach is often preferable compared to using ad-hoc $M / N$ rules [25].

\subsubsection{Assignment Problem}

Finding likely assignments of new measurements to existing tracks, new tracks and false detections is commonly called the assignment problem. Given is a set $\mathcal{T}$ of $N$ tracks that are the result of previous measurements and a hypothesis, and a set $\mathcal{Y}=\left\{\mathbf{y}_{i}\right\}_{i=1}^{M}$ of new measurements. See Figure 5.1 for a simple scenario involving three tracks and two measurements that will be used to illustrate the concepts in this section. The goal is to assign each measurement to either one of the existing tracks, a new track or identify it as false detection. The measurements are first gated for all tracks, resulting in a gating matrix, and then association probabilities are computed for all possible associations, resulting in an association matrix.

The gating matrix $\mathbf{G}$ is a logical matrix with one measurement per column and one track per row. If a measurement is gated by a track, the corresponding element is set to true, otherwise it is set to false. An example of a gating matrix is shown in Table 5.1. An equivalent representation is the set $\mathcal{G}_{\tau}$ containing the indices $i \in\{1: M\}$ gated by the track $\tau \in \mathcal{T}$. Similarly, the association matrix $\mathbf{A}$ has one measurement per column and one track per row, with additional rows corresponding to false detections and new tracks. An example of an association matrix is shown in Table 5.2 .

An assignment can be represented as a matrix $\mathbf{M}$ of dimension $(N+2 M) \times M$, where elements are either zero or one. The matrix has exactly one non-zero element per column, $\sum_{l} \mathbf{M}_{l, i}=1 \forall i$, and at most one non-zero element per row, 
Table 5.1: An example of a gating matrix for two measurements and three tracks, where the symbol $\checkmark$ indicates that the measurement is within the gate of the track.

\begin{tabular}{l|cc} 
& Measurement $\mathbf{y}_{1}$ & Measurement $\mathbf{y}_{2}$ \\
\hline Track $\tau_{1}$ & $\checkmark$ & \\
Track $\tau_{2}$ & $\checkmark$ & $\checkmark$ \\
Track $\tau_{3}$ & & $\checkmark$ \\
\hline
\end{tabular}

Table 5.2: An example of an association matrix for two measurements and three tracks, where each element contains the log probability contributed to the hypothesis score if the measurement is associated to the track. The bottom rows are for associations to false detections and new tracks. The symbol $\times$ indicates that association is not to be considered according to the gating matrix, corresponding to a contribution of negative infinity.

\begin{tabular}{r|cc} 
& Measurement $\mathbf{y}_{1}$ & Measurement $\mathbf{y}_{2}$ \\
\hline Track $\tau_{1}$ & $\log \frac{P_{D} p\left(\mathbf{y}_{1} \mid \tau_{1}\right)}{1-P_{D} P_{G}}$ & $\times$ \\
Track $\tau_{2}$ & $\log \frac{P_{D} p\left(\mathbf{y}_{1} \mid \tau_{2}\right)}{1-P_{D} P_{G}}$ & $\log \frac{P_{D} p\left(\mathbf{y}_{2} \mid \tau_{2}\right)}{1-P_{D} P_{G}}$ \\
Track $\tau_{3}$ & $\times$ & $\log \frac{P_{D} p\left(\mathbf{y}_{2} \mid \tau_{3}\right)}{1-P_{D} P_{G}}$ \\
False Detection & $\log \left(\beta_{f}\right)$ & $\times$ \\
False Detection & $\times$ & $\log \left(\beta_{f}\right)$ \\
New Track & $\log \left(\beta_{n}\right)$ & $\times$ \\
New Track & $\times$ & $\log \left(\beta_{n}\right)$ \\
\hline
\end{tabular}

$\sum_{i} \mathbf{M}_{l, i} \leq 1 \forall l$, where $\mathbf{A}_{a, b}$ denotes the element at row $a$ and column $b$. The motivation for this structure is the assumptions that each measurement is generated by at most one target and that each target generates at most one measurement. An equivalent and more compact representation is a vector $\mathbf{m}$ of dimension $M$ where each element corresponds to a measurement and points to the associated track, a false detection or a new track.

The assignment problem, formulated using the matrix representation, is then defined as

$$
\begin{array}{lll}
\underset{\mathbf{M}}{\operatorname{minimize}} & \sum_{l} \sum_{i} \mathbf{M}_{l, i} \mathbf{A}_{l, i}, & \\
\text { subject to } & \sum_{l} \mathbf{M}_{l, i}=1, & \forall i, \\
& \sum_{i} \mathbf{M}_{l, i} \leq 1, & \forall l, \\
& \mathbf{M}_{l, i} \in\{0,1\}, & \forall l, i .
\end{array}
$$

There are many algorithms available to solve this problem, e.g., the Hungarian 
method [80], Munkres algorithm [24], Jonker-Volgenant relaxation [71], the auction algorithm $[20,21,34]$ and the Jonker-Volgenant-Castanón algorithm [43]. The auction algorithm is considered the most efficient in [25]. To find a number of the best solutions to the assignment problem Murty's method can be used [37, $38,91]$.

\subsection{Multiple Hypothesis Tracker}

The multiple hypothesis tracker MHT, described in [25], is designed for MTT. The tracker results in optimal performance in its non-approximated form. However, as mentioned previously, the tracker is intractable due to the combinatorial explosion of the number of hypotheses to consider, which grows exponentially in time. Several methods exist for handling the intractability in a suboptimal manner. One common approach is to reduce the number of maintained hypotheses, through pruning of low-probability hypotheses and merging of similar hypotheses.

There are two common approaches to MHT [25], hypothesis oriented and track oriented. In the former, a number of the most likely hypotheses are maintained at each time step, where each hypothesis consists of its own set of tracks. This is the original approach, presented in [98], and it is relatively simple to implement. The main drawback, however, is that multiple copies of identical tracks might be maintained in different hypotheses. The latter instead maintains a set of distinct tracks and constructs hypotheses at each time step of non-conflicting tracks. Hybrids of the two approaches can also be considered.

The MHT presented here is hypothesis oriented, where a set of the $N_{H}$ most likely hypotheses are maintained. At the reception of new measurements, the $N_{H}$ new most likely hypotheses are constructed from each maintained hypothesis, resulting in $N_{H}^{2}$ hypotheses. Of these, the $N_{H}$ most likely hypotheses are maintained for the next time step.

The algorithm for the tracker is outlined in Algorithm 5.1. A hypothesis is represented by a set of tracks and a probability. Given a hypothesis with a set of tracks and associated measurements, estimation in the tracker is performed using the time update and measurement update steps in, e.g., the KF or EKF. To reduce the number of considered associations, a gating matrix is constructed for each hypothesis as described in Section 5.1.4. Given the predicted tracks, the measurements and the gating matrix an association matrix is computed for each hypothesis as described in Section 5.1.4. The assignment problem is then solved for each hypothesis using Murty's algorithm to find a number of the most likely new hypotheses and their probabilities, as discussed in Section 5.1.4.

The MHT is used in the savannah application and the results are shown in Application 5.1 . 


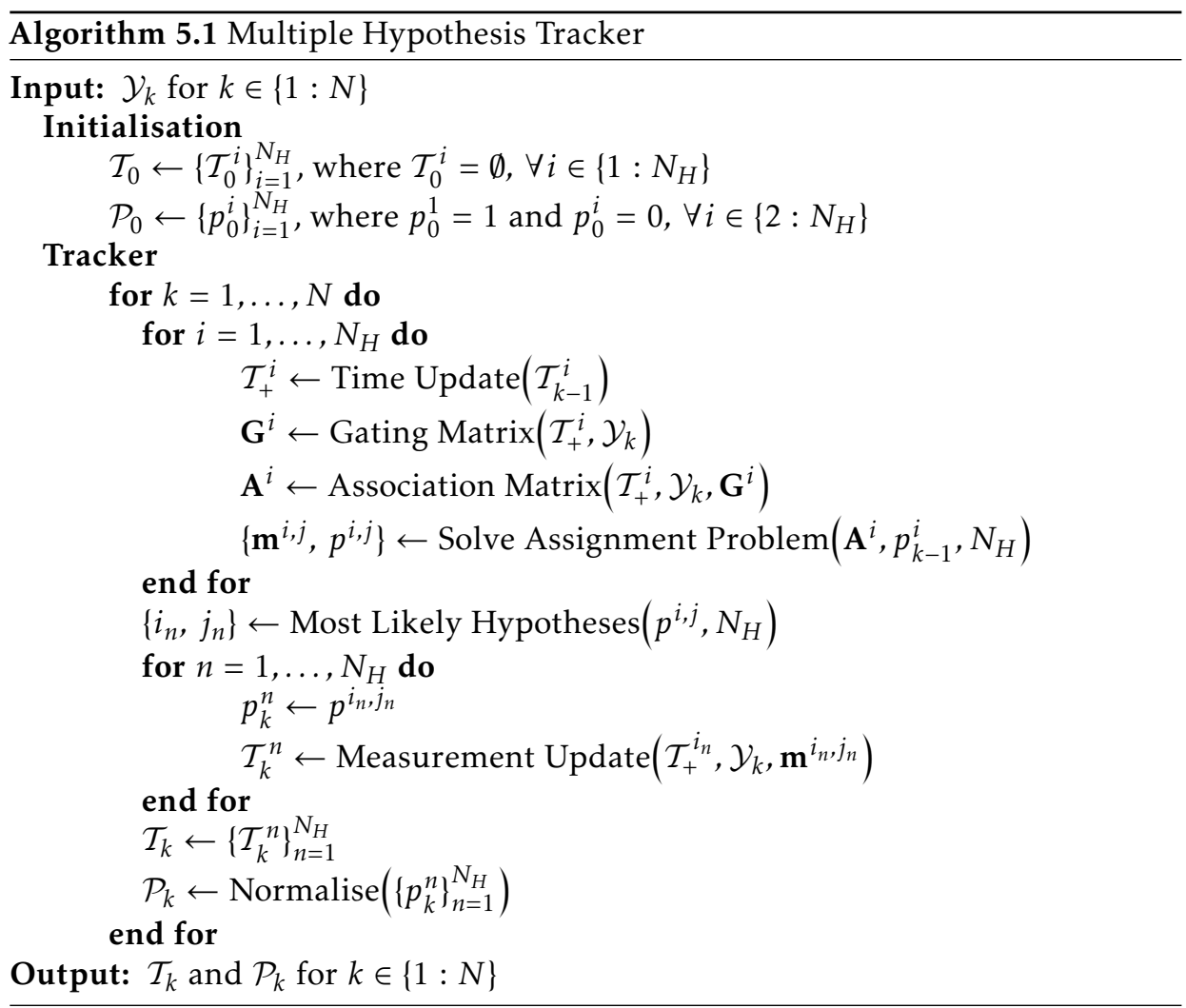

Application 5.1: Savannah Animal Tracking

Savannah animals are tracked in image coordinates as a quadcopter flies above the savannah at Kolmården Wildlife Park. The model used for the animals is described in Application 2.10, but the origins of the measurements are uncertain. To handle the uncertainty the MHT is applied as outlined in Algorithm 5.1, with the time and measurement updates replaced by (4.34) and (4.35), respectively. Figure 5.2 shows an outline of the elements included in the tracker. $M / N$-logic is used for deletion of tracks and Murty's algorithm with the auction algorithm is used to solve the assignment problem.

An example of the results from the MHT is shown in Figure 5.3. It is obvious that some targets are unresolved. This issue results from the measurement preprocessing and is not corrected by the tracker.

Extending the algorithm to tracking in world coordinates would result in several additional challenges. Since measurements are only obtained in two dimensions, estimation of the extent in three dimensions would require a different extension model or modifications to the model considered in this thesis. A ground model would be necessary for relatively accurate position estimates of the animals. Considering the large area to be covered, better identification of the animals, or at 


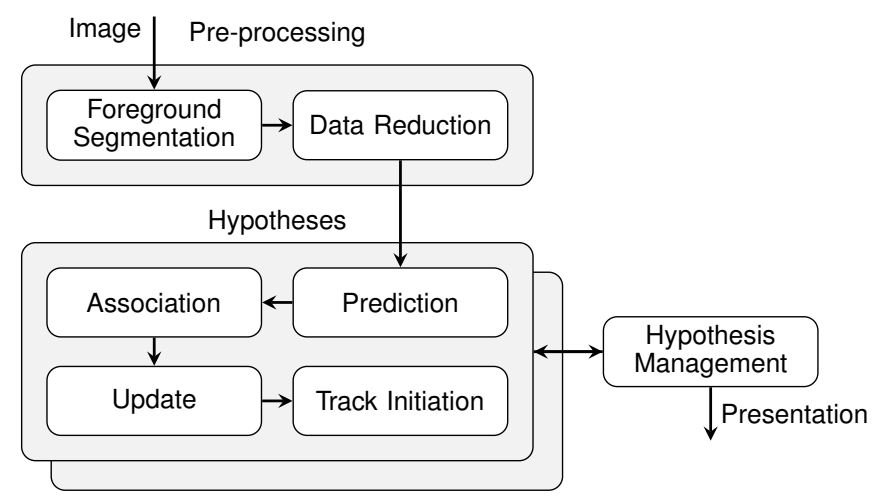

Figure 5.2: The pipeline of processing a frame from the sensor in the savannah application.

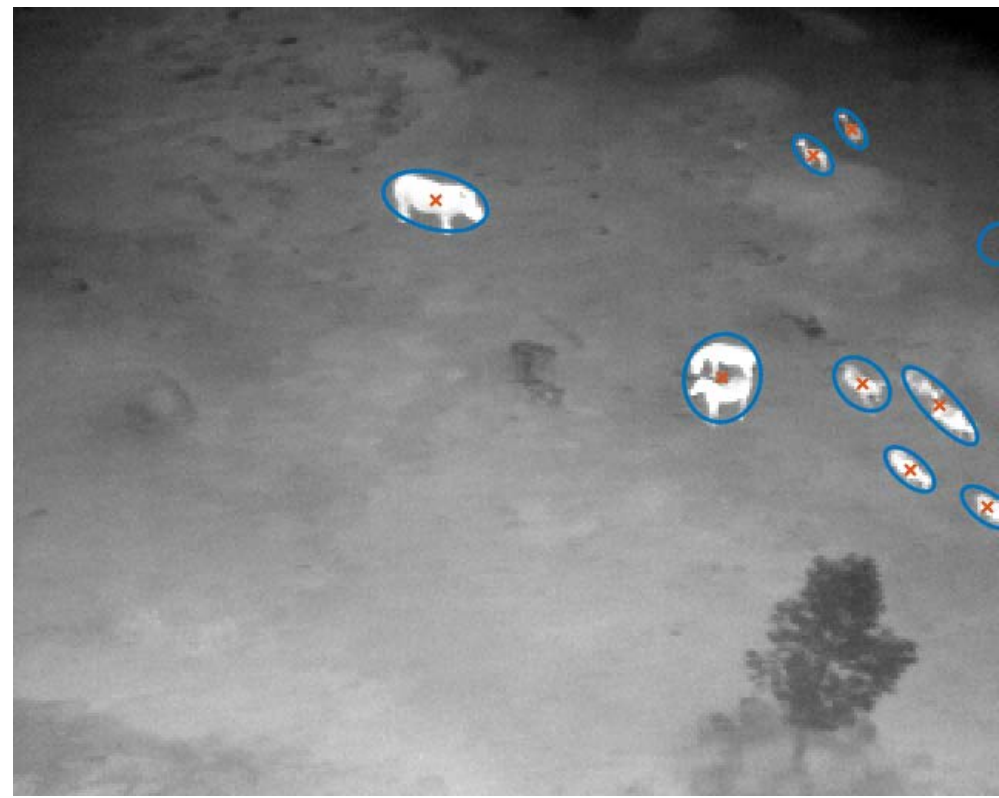

Figure 5.3: An example of the results from the MHT. The red cross shows the position of the target and the blue ellipse shows the estimated extent based on a uniform measurement distribution. 
least classification of the types of animals, would be needed to reduce data association uncertainty. The position of the camera would need to be estimated as well as a camera model. Occlusions from terrain or vegetation would need to be considered.

\subsection{Probabilistic Data Association Filter}

Considering the high computational complexity of the MHT, in applications with few targets, high probability of detection and few false detections a less demanding filter often achieves similar performance. One such filter is the PDA filter, originally presented in [13], which is an STT filter. The approach is to consider multiple hypotheses for association and merge them into a single hypothesis at each time step, as described in [11] and [25]. An MTT extension to the filter, the JPDA filter [11, 49], is designed for improved performance for scenarios with multiple point targets.

The conventional PDA filter is described in Section 5.3.1. Track management for the PDA filter is described in Section 5.3.2 as well as a proposed method for merging similar tracks. Section 5.3.3 presents a modification to the PDA filter, previously published by the author in [112], that allows for estimation of the target extent.

\subsubsection{Standard Probabilistic Data Association Filter}

In this section the PDA filter is described briefly. See [25] for a more thorough description.

Given is a set $\mathcal{T}$ of $N$ tracks and a set $\mathcal{Y}=\left\{\mathbf{y}_{i}\right\}_{i=1}^{M}$ of new measurements. The predicted distributions of the state and measurement for a track $\tau \in \mathcal{T}$ is given by $p(\mathbf{x} \mid \tau)=\mathcal{N}\left(\mathbf{x} \mid \hat{\mathbf{x}}^{\tau}, \mathbf{P}^{\tau}\right)$ and $p(\mathbf{y} \mid \tau)=\mathcal{N}\left(\mathbf{y} \mid \hat{\mathbf{y}}^{\tau}, \mathbf{S}^{\tau}\right)$, respectively. Note that the time index is omitted for notational convenience.

Gating is first performed as described in Section 5.1.1 for the measurements $\mathcal{Y}$ and tracks $\mathcal{T}$. This results in the sets $\mathcal{G}_{\tau}$ defined in Section 5.1.4. The number of measurements gated to track $\tau$ is given by $M_{\tau}=\left|\mathcal{G}_{\tau}\right|$. For each track $\tau \in \mathcal{T}$, hypotheses $\theta_{\tau}^{i}$ are constructed for all $i \in \mathcal{G}_{\tau}$. Hypothesis $\theta_{\tau}^{i}$ states that the gated measurement $\mathbf{y}_{i} \in \mathcal{Y}$ is generated by the target and all other measurements in the gate are false detections. The probability of such a hypothesis for the LGSSM is

$$
\begin{aligned}
\mu_{i}^{\tau} \triangleq p\left(\theta_{\tau}^{i} \mid \mathcal{Y}, \tau\right) & \propto \beta^{M_{\tau}-1} P_{D} p\left(\mathbf{y}_{i} \mid \theta_{i}^{\tau}, \tau\right) \\
& =\beta^{M_{\tau}-1} P_{D} \mathcal{N}\left(\mathbf{y}_{i} \mid \hat{\mathbf{y}}^{\tau}, \mathbf{S}^{\tau}\right),
\end{aligned}
$$

where $\beta=\beta_{f}+\beta_{n}$. In addition, a hypothesis $\theta_{\tau}^{0}$ is constructed that all measurements in the gate are false detections, with probability

$$
\mu_{0}^{\tau}=p\left(\theta_{0}^{\tau} \mid \mathcal{Y}, \tau\right) \propto \beta^{M_{\tau}}\left(1-P_{D} P_{G}\right) .
$$


The hypothesis probabilities $\mu_{i}^{\tau}$ are normalised over $i \in \mathcal{G}_{\tau} \cup 0$. The posterior distribution of the state for each track $\tau \in \mathcal{T}$ is

$$
\begin{aligned}
p(\mathbf{x} \mid \tau, \mathcal{Y}) & =\sum_{i \in \mathcal{\mathcal { G } _ { \tau }} \cup 0} p\left(\mathbf{x}, \theta_{i}^{\tau} \mid \tau, \mathcal{Y}\right)=\sum_{i \in \mathcal{\mathcal { G } _ { \tau } \cup 0}} p\left(\theta_{i}^{\tau} \mid \tau, \mathcal{Y}\right) p\left(\mathbf{x} \mid \theta_{i}^{\tau}, \tau, \mathcal{Y}\right) \\
& =\sum_{i \in \mathcal{\mathcal { G } _ { \tau }} \cup 0} \mu_{i}^{\tau} \mathcal{N}\left(\mathbf{x} \mid \hat{\mathbf{x}}_{i}^{\tau}, \mathbf{P}_{i}^{\tau}\right),
\end{aligned}
$$

which is a mixture of Gaussians. The last factor, $\mathcal{N}\left(\mathbf{x} \mid \hat{\mathbf{x}}_{i}^{\tau}, \mathbf{P}_{i}^{\tau}\right)$, is obtained by performing a measurement update for the track $\tau$ in the KF in Algorithm 4.2 with the associated measurement $\mathbf{y}_{i}$. For the hypothesis $\theta_{0}^{\tau}$ the prediction distribution for the track is used. The merged hypothesis is obtained by matching the first two moments of the distribution to a Gaussian, resulting in

$$
p(\mathbf{x} \mid \tau, \mathcal{Y}) \approx \mathcal{N}\left(\mathbf{x} \mid \hat{\mathbf{x}}_{+}^{\tau}, \mathbf{P}_{+}^{\tau}\right)
$$

where

$$
\hat{\mathbf{x}}_{+}^{\tau}=\sum_{i \in \mathcal{G}_{\tau} \cup 0} \mu_{i}^{\tau} \hat{\mathbf{x}}_{i}^{\tau}
$$

and

$$
\mathbf{P}_{+}^{\tau}=\sum_{i \in \mathcal{G}_{\tau} \cup 0} \mu_{i}^{\tau}\left(\mathbf{P}_{i}^{\tau}+\left(\hat{\mathbf{x}}_{i}^{\tau}-\hat{\mathbf{x}}_{+}^{\tau}\right)\left(\hat{\mathbf{x}}_{i}^{\tau}-\hat{\mathbf{x}}_{+}^{\tau}\right)^{T}\right)
$$

\subsubsection{Track Management}

To initiate new tracks, confirmed and tentative tracks are treated separately. The steps in Section 5.3 are performed in two levels. The first level is for all confirmed tracks and the second level is for all tentative tracks using the non-gated measurements. Any remaining non-gated measurements are considered to be false detections or new tracks. These are used to initiate new tentative tracks. Tentative tracks are either confirmed or deleted based on the track quality as described in Section 5.1.3.

Track management also constitutes merging of similar tracks. The motivation for merging similar tracks is to improve the performance of the PDA filter. One such method is described in this section. Similar tracks $\tau_{a}$ and $\tau_{b}$ are merged based on the Bhattacharyya distance [22]

$$
d_{B}\left(\tau_{a}, \tau_{b}\right)=\frac{1}{4}\left(\hat{\mathbf{x}}^{\tau_{a}}-\hat{\mathbf{x}}^{\tau_{b}}\right)^{T}\left(\mathbf{P}^{\tau_{a}}+\mathbf{P}^{\tau_{b}}\right)^{-1}\left(\hat{\mathbf{x}}^{\tau_{a}}-\hat{\mathbf{x}}^{\tau_{b}}\right)+\frac{1}{2} \ln \left(\frac{\left|\left(\mathbf{P}^{\tau_{a}}+\mathbf{P}^{\tau_{b}}\right) / 2\right|}{\sqrt{\left|\mathbf{P}^{\tau_{a}}\right|\left|\mathbf{P}^{\tau_{b}}\right|}}\right),
$$

which measures the similarity between two multivariate normal distributions. If a set of tracks $\mathcal{T}$ satisfies $d_{B}\left(\tau_{a}, \tau_{b}\right) \leq \gamma_{m}$ for all $\tau_{a}, \tau_{b} \in \mathcal{T}$, where $\gamma_{m}$ is a design parameter, the tracks are merged. Tracks are merged using [100]

$$
\hat{\mathbf{x}}^{+}=\sum_{\tau \in \mathcal{T}} w_{\tau} \hat{\mathbf{x}}^{\tau}
$$




$$
\mathbf{P}^{+}=\sum_{\tau \in \mathcal{T}} w_{\tau}\left(\mathbf{P}^{\tau}+\left(\hat{\mathbf{x}}^{\tau}-\hat{\mathbf{x}}^{+}\right)\left(\hat{\mathbf{x}}^{\tau}-\hat{\mathbf{x}}^{+}\right)^{T}\right),
$$

where the weight $w_{\tau}=\left|\mathbf{P}^{\tau}\right| / \sum_{\tau \in \mathcal{T}}\left|\mathbf{P}^{\tau}\right|$ is chosen to prioritize tracks with a large extent. The posterior distribution of the track with the largest weight is replaced by $\mathcal{N}\left(\hat{\mathbf{x}}^{+}, \mathbf{P}^{+}\right)$and the remaining tracks are deleted.

\subsubsection{Modified Probabilistic Data Association Filter}

A modification to the PDA filter is presented in this section as previously published by the author in [112]. A side effect of the PDA filter when receiving many measurements with high covariance near a track is that the state covariance grows, and consequently also the covariance of the measurement prediction. Therefore, by a certain abuse of the filter, an estimate of the extent of the target can be obtained from the measurement distribution of the track without explicitly modelling the extent.

By considering the reduced and mapped measurements in (3.23), restated here for convenience,

$$
\mathcal{Y}=\left\{\mathbf{y}_{i}, d_{i}, \mathbf{Y}_{i}\right\}_{i=1}^{M},
$$

the PDA filter can be modified to estimate the extent and improve tracking performance in certain scenarios. Each measurement in the set corresponds to a number of segmented foreground pixels, which can be seen as individual detections of the target. The number of individual measurements represented by each mapped reduced measurement can be approximated by the size of the covariance matrix

$$
n_{i}=\left|\mathbf{Y}_{i}\right|,
$$

or by the size weighted by the degree of confidence, as

$$
n_{i}=d_{i}\left|\mathbf{Y}_{i}\right| .
$$

Each gated measurement $\mathbf{y}_{i}$ for track $\tau \in \mathcal{T}$, such that $i \in \mathcal{G}_{\tau}$, is used to update the PDA filter $n_{i}$ times, which results in the weights, with $n_{0}=1$,

$$
\mu_{i}^{\tau} \propto n_{i} p\left(\theta_{i}^{\tau} \mid \mathcal{Y}, \tau\right), \quad i \in \mathcal{G}_{\tau} .
$$

As argued in Section 4.5 a uniform distribution is a more reasonable assumption compared to a Gaussian distribution for the measurements in a camera sensor. The hypothesis probabilities in (5.13) are modified such that true measurements are uniformly distributed within the gate, resulting in

$$
p\left(\theta_{i}^{\tau} \mid \mathcal{Y}, \tau\right) \propto \frac{\beta^{M_{\tau}-1} P_{D}}{V}=\frac{\beta^{M_{\tau}-1} P_{D}}{\pi\left|4 \mathbf{S}^{\tau}\right|}, \quad \quad i \in \mathcal{G}_{\tau},
$$

where $V$ is the volume of the gate and Proposition 4.1 is used, and

$$
p\left(\theta_{0}^{\tau} \mid \mathcal{Y}, \tau\right) \propto \beta^{M_{\tau}}\left(1-P_{D}\right)
$$

When the number of gated measurements $M_{\tau}$ is large, the probability $\mu_{0}^{\tau}$ of hy- 
pothesis $\theta_{0}^{\tau}$ approaches zero. The weights can then be approximated by

$$
\mu_{i}^{\tau} \approx \frac{n_{i}}{\sum_{i \in \mathcal{G}_{\tau}} n_{i}}, \quad i \in \mathcal{G}_{\tau} .
$$

Application 5.2 compares the standard and modified PDA filters for the dolphin application as well as the different multiplicities in (5.19). The comparison was previously published by the author in [112].

\section{Application 5.2: Dolphin Filter Comparison}

The PDA filter is compared to the modified PDA filter proposed in this section for the dolphin application. Also, the two different multiplicities in (5.19) are compared for the modified PDA filter.

Targets cast shadows, as seen in Figure 3.5, which often has a lower degree of confidence than the targets. Using only size as in (5.19a) to determine the measurement multiplicity, all foreground measurements will have similar weights in the target tracking filter, while including the degree of confidence as in (5.19b) puts higher weights on true targets than shadows. To compare the two options the results in the presence of the shadow in part A in Figure 3.5 is shown in Figure 5.4. Using only size, the trajectory is sensitive to the shadow, since the same weight is put on all measurements. However, when including using both size and degree of confidence in the multiplicity the shadow measurements have less impact. This gives a smoother trajectory estimate and an innovation covariance that mainly covers the target. The side effect is that a new track is initiated on the shadow, which, is subsequently merged into the original track with little effect to its trajectory.

For the PDA filter described in Section 5.3 the measurements are assumed to be Gaussian distributed around the target position, effectively giving more weight to measurements near the centroid. This results in poor estimation of the target extent. To mitigate this effect, the modified PDA filter in Section 5.3.3 is used instead. It assumes uniformly distributed measurements in the gate as proposed in (5.21) and utilises the multiplicity of the measurements as in (5.20). To compare the two options the filter performances are evaluated in a sharp turn. The results are given in Figure 5.5. The standard PDA filters struggle to track the target through the turn for various choices of process and measurement noise covariances, while the modified PDA filter finds the centroid. It also adapts the covariance to match the extent of the target, improving the performance.

The proposed modifications in the modified PDA filter are relatively minor, but are shown to perform better in certain scenarios. A noticeable problem with the proposed method is that the extent estimate grows with missed detections, since the state covariance is reused. This could be mitigated by using an explicit extent model, e.g., as described in Section 2.6. Similar modifications to handle uniformly distributed measurements and degree of confidence could be applied for this model as well. 


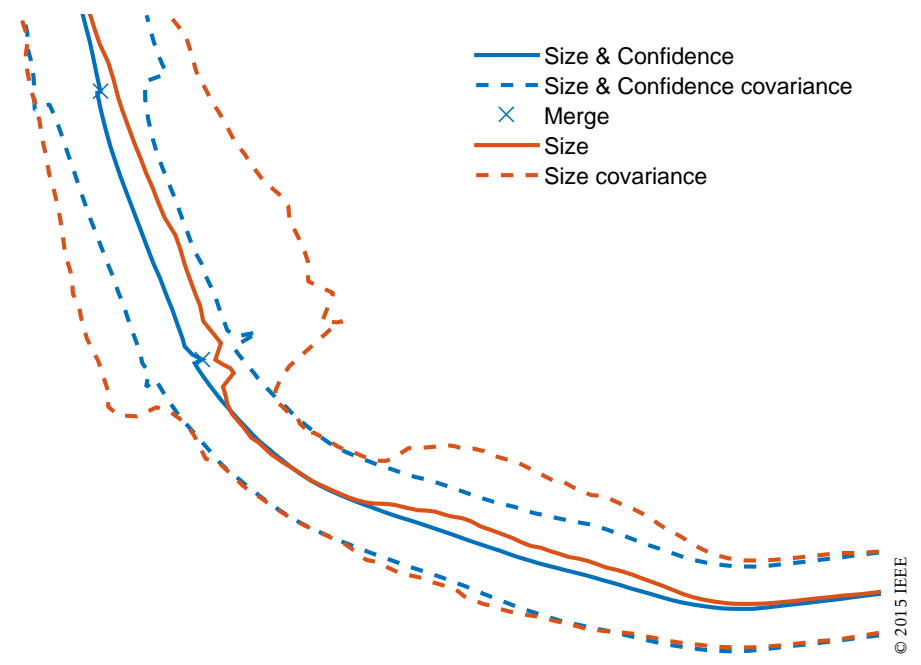

Figure 5.4: Compares the estimated trajectory and the estimated extent in the presence of a shadow using size and degree of confidence as well as only size as multiplicity of the measurements. The estimated extent for the former trajectory is less sensitive to the shadow. Published in [112].

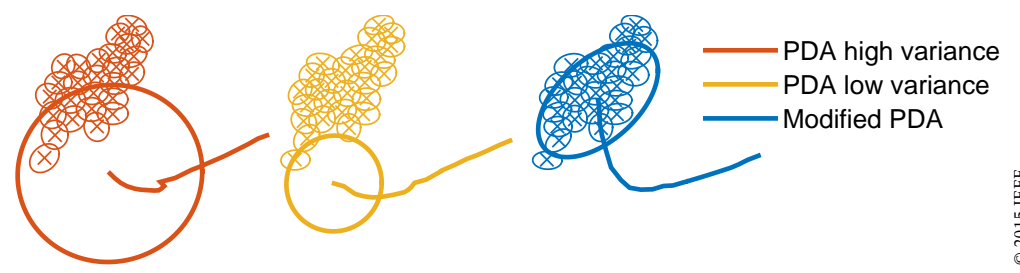

Figure 5.5: Comparison of the estimated centroid and extent for the standard PDA filter, using high and low Gaussian measurement noise covariance, with the modified PDA filter during a sharp turn. Published in [112]. 


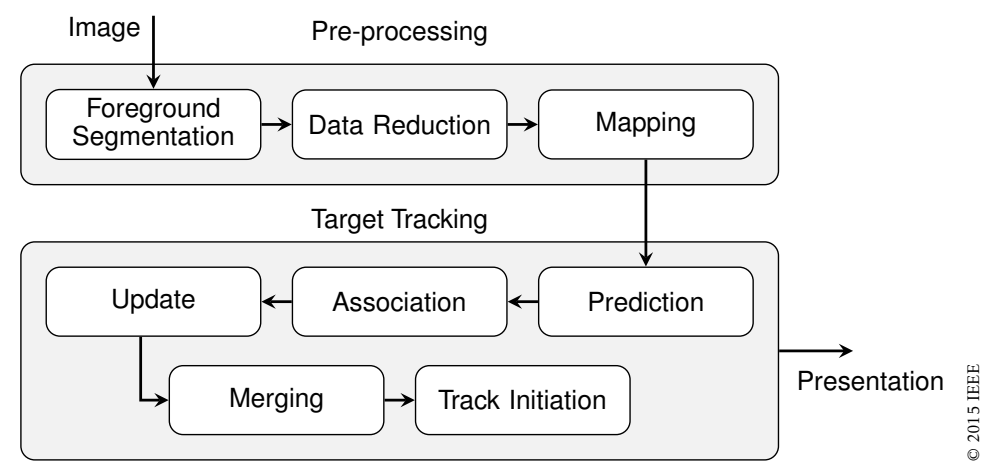

Figure 5.6: The pipeline of processing a frame from the sensor in the dolphin application. Track Initiation essentially consists of an additional target tracking module for tentative tracks. Published in [112].

In Application 5.3 the full framework is put together for tracking in the dolphin application. Some discussion regarding the results is presented. The framework and the results were previously published by the author in [112].

\section{Application 5.3: Dolphin Framework}

The full framework for tracking the dolphins is put together. The pre-processing of the measurements, as presented in Application 3.3 for foreground segmentation and in Applications 3.2 and 3.6 for measurement reduction and mapping, is utilised. The SSM considered is presented in Applications 2.3 and 2.5.

Detecting and tracking the dolphins comes with many uncertainties, e.g., number of targets, false detections, missing detections, unresolvable targets and association uncertainty among others. Some methods to handle these uncertainties are discussed in this chapter. In Section 3.4 each dolphin generates a number of detections, while the assumption for the estimation is that the target can generate at most one detection at each time step. This is utilised in the modified PDA filter.

The initial estimate of the position of the dolphin is taken as the initial measurement, and the velocity is initially set to zero, $\overline{\mathbf{x}}_{0}=\left(\mathbf{y}_{1}^{T}, \mathbf{0}^{T}\right)^{T}$. Since the position is initialised with information about the target, the initial covariance $\mathbf{P}_{0}$ is chosen to be diagonal and relatively small.

The motion model of the dolphin is given by (2.31) and (2.25) and the sensor model is given by (2.37). The EKF in Algorithm 4.2 is applied to the sequence of measurements using these models. The measurement update step is omitted when no measurement is available. The linearisation around the state of the motion model is obtained from (2.33). To handle the data association uncertainty, the modified PDA filter presented in this section is used with track management as described in Section 5.3.2. The measurement set in Application 3.6 is used in the filter, and (5.19b) is used as multiplicity. Figure 5.6 shows an outline of the elements included in the tracker. 
The trajectory resulting from the filter for one group of dolphins is shown in Figure 5.7. Several tracks are merged into a single track along the way. Although not showing in the figure, several individuals also leave the group along the trajectory, for which new tracks are initiated. The advantage of the constrained motion model is apparent at the bottom left where the target is occluded for over 100 frames while the track is maintained. It is apparent from the mapped detection region that the mapping is inaccurate in some areas. This is a result of an inaccurate structure for the camera model or issues related to the estimation of the camera model.

The framework is applied to five minute sequences recorded at Kolmården Wildlife Park and outputs a set of trajectories. The main difficulty is the handling of dolphins entering and leaving groups, which provides difficulties for the tracking algorithm as well as for the presentation of the results. An alternative to presenting individual tracks is to aggregate all estimated tracks into a map to show where the dolphins spend most time. Figure 5.8 shows such a map where preferred regions and trajectories are clearly visible. This could be presented to the biologists as a supplement to the individual trajectories for a quick overview. 




Figure 5.7: A track of a group of targets, with individuals joining and splitting from the group. The blue circles show initiated tracks that are merged with the long track. The dashed blue line shows the constraint region and the dashed red line shows the mapped detection region. The filter succeeds in maintaining the track while the targets move outside of the detection region at the bottom right and when the target disappears for a long time in the non-detection region at the bottom left. Published in [112].

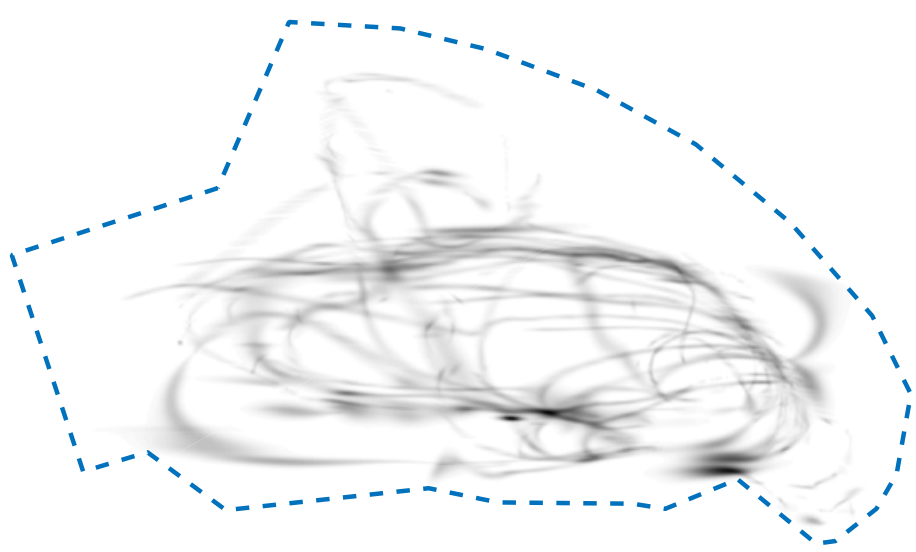

Figure 5.8: A map showing the aggregated estimates of all dolphins for a five minute sequence. Although individual tracks are discarded, the behaviour of the dolphins can be analysed from such presentations. The dashed blue line shows the constraint region. 



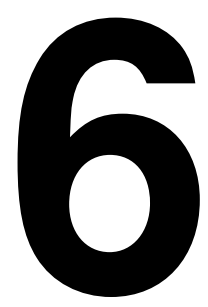

\section{Conclusions and Future Work}

A framework for target tracking has been presented in this thesis. The framework is decomposed into the elements [25]:

- pre-processing of sensor data to obtain detections;

- association between detections and predictions for existing tracks;

- estimation for each track using associated detections; and

- track management.

Select established methods for each element of the framework were presented and some problems were considered in more detail. Theory was developed for three particular problems selected from a handful applications, which constitute the main contributions of the thesis:

1. The development and analysis of a model with observations sampled at an uncertain time.

2. The development of a constrained motion model that results in improved and feasible predictions of certain targets constrained to a region.

3. The development of an extension to the jump Markov model incorporating state-independent measurements that directly relate to the mode.

The uncertain timestamp model was presented in Section 2.7 for a single observation of uncertain time in a linear Gaussian state-space model. The posterior smoothing distributions as well as various common point estimators for the states and the uncertain time were derived in Section 4.6. The implications of the additional observation were analysed for a simple scenario and the results from the analysis were presented in Section 4.6. To demonstrate the developed theory the 
model was applied in an orienteering application, and the results were presented in Application 4.4.

The constrained motion model was developed in Section 2.3. The model results in feasible predictions of a target known to be inside a constraint region, which is an improvement over conventional motion models. The model was applied in a dolphin tracking application and the results of using the model with an extended Kalman filter were presented in Applications 2.4 and 5.3.

The extension to the jump Markov model was developed in Section 2.5.2 and estimation for the model using a filter bank was developed in Section 4.4.2. The model was applied in a bird tracking application, where additional measurements were used to improve the estimation of the bird being in stationary or flight mode. The results for the application were presented in Application 4.1 and a comparison to a manual method was performed.

Four applications and one scenario were presented in this thesis. Relevant background theory and new theory and methods necessitated by problems in the applications were presented. Solutions to the applications were presented throughout the thesis intermixed with theory. Results for each application and the scenario were presented.

A few steps towards providing solutions to problems facing biologists and park rangers have been taken. Two applications presented in this thesis provide biologists with solutions for automating data collection. Similar solutions could potentially be applied for ground cameras in wildlife reserves. An application involving animal tracking using an airborne camera was also attempted for tracking savannah animals. First steps towards incorporating sensor data with witness reports from park rangers were taken in the form of an evaluation of a simplified scenario and an implementation in a similar application.

For the dolphin application a complete solution has been presented. An established method for background estimation was developed slightly and tailored to the application in Section 3.3. A method to calibrate a camera using available data and a map was developed in Section 3.2. A method to reduce and map the large number of detections in the video was presented in Section 3.4. A novel motion model for dolphins in captivity was developed by the author in Section 2.3 that maintains predictions of target motion inside the basin. An adaptation of a standard target tracking filter was presented in Section 5.3.3 that allows for estimation of the extent of the dolphins. Results for the complete framework were presented in Application 5.3. Further work for this application would be a more robust estimation procedure for the camera model and replacing the target tracking filter with a state-of-the-art tracker that explicitly models the extents of the dolphins. Benefits could also be obtained from incorporating the camera model into the state-space model compared to the current solution of mapping measurements during pre-processing. The handling of group targets needs to be improved. 
For the bird application a complete solution has been presented. The bird's behaviour while stationary and during flight was modelled as modes in a jump Markov model in Section 2.5. Additional information extracted using image processing was also considered in the model, as developed by the author in Section 2.5.2, to improve the estimation of the bird position and mode. Estimation in the model extension and some heuristic methods for a more robust estimation of takeoff times and directions were developed in Section 4.4. A stationary camera model was also presented in Section 3.2 and estimated using only available data and the shape of an object in the scene. Results from the method were also compared to another method applied to the same data in Application 4.1, showing that the proposed method is a viable option for biologists to improve their traditional methods. Further work for this application would be to develop more robust and adaptive image processing methods to reduce sensitivity to varying experiment setups, as well as automatic detection of the funnels in the scene. More information from the video, such as the bird's extent, could also be incorporated into the jump Markov model and an improved analysis of the implication of the model extension would be interesting. A smoother for the model would be preferable to the currently used filter.

For the savannah application, a part solution has been obtained. The position and extension of the animals in image coordinates were modelled using established methods in Section 2.6 and estimation for the model was presented in Section 4.5. To handle the association uncertainty the multiple hypothesis tracker presented in Section 5.2 was applied. Results from tracking the savannah animals were presented in Application 5.1. No new theory or method has been developed for this application, but the application serves as a first venture for the author into stateof-the-art algorithms. Further work for this application would require collection of new data including the position and orientation of the camera synchronised with the video to allow for tracking in world coordinates. A ground model would be required as well for accurate positioning.

For the simple uncertain time scenario the posterior smoothing distributions and some point estimators have been obtained for a one-dimensional state-space model with an observation sampled at an uncertain time. A linear Gaussian statespace model with an observation sampled at an uncertain time was presented in Section 2.7. The posterior smoothing distributions as well as various common point estimators for the states and the uncertain time were derived in Section 4.6. The impact of the additional observation was analysed using a one-dimensional state-space model in Section 4.6. Further work for this scenario would be to consider several observations with an uncertain timestamp. Also the corresponding filtering distribution and the impact of nonlinear state-space models would be of interest in some applications. An alternative to considering the discrete problem would also be to derive the solution in continuous time.

The orienteering application is based on the uncertain timestamp model developed by the author in Section 2.7 and estimation for such models as derived in Section 4.6. Results for the application were presented in Application 4.4. The 
estimated position of the sprinter improved slightly when including the observation with an uncertain timestamp. Only a small segment of the race involving a single control point was considered. Further work for this scenario would apply the method to the entire race for multiple control points. Also a filter based on the model would be interesting to improve the estimated position online.

The two strongest candidates for continuing the work is found in further development of the uncertain time model as well as further collaborations with Kolmården Wildlife Park to collect data and develop the solution for airborne tracking of savannah animals. 


\section{Bibliography}

[1] R. A. Adams. Calculus: A Complete Course. Pearson Education Canada, Toronto, Ontario, Canada, fifth edition, 2003. ISBN 978-0-201-79131-0. Cited on page 77 .

[2] J. Ajgl, M. Šimandl, and J. Duník. Millman's formula in data fusion. In Proceedings of the 10th International PhD Workshop Young Generation Viewpoint, pages 1-6, Hluboka and Vltavou, Czech Republic, Sept. 2009. Cited on page 85 .

[3] A. G. Akritas, E. K. Akritas, and G. I. Malaschonok. Various proofs of Sylvester's (determinant) identity. Mathematics and Computers in Simulation, 42(4-6):585-593, Nov. 1996. ISSN 0378-4754. Cited on page 85.

[4] B. D. O. Anderson and J. B. Moore. Optimal Filtering. Dover Publications, Mineola, NY, USA, 1979. ISBN 978-0-486-43938-9. Cited on page 10.

[5] K. J. Åström. Introduction to Stochastic Control Theory. Dover Publications, Mineola, NY, USA, 2006. ISBN 978-0-486-44531-1. Cited on pages 19,20 , and 21 .

[6] M. Athans and C.-B. Chang. Adaptive estimation and parameter identification using multiple model estimation algorithm. Technical Report ESD-TR76-184, Massachusetts Institute of Technology, Lexington, MA, USA, 1976. Cited on page 10.

[7] D. J. Ballantyne, H. Y. Chan, and M. A. Kouritzin. Novel branching particle method for tracking. In Proceedings of SPIE, volume 4048, pages 277-287, 2000. doi:10.1117/12.391984. Cited on page 9.

[8] I. Y. Bar-Itzhack and Y. Vitek. The enigma of false bias detection in a strapdown system during transfer alignment. Journal of Guidance, Control, and Dynamics, 8(2):175-180, 1985. Cited on page 15.

[9] Y. Bar-Shalom, editor. Multitarget-Multisensor Tracking: Applications and Advances, volume II. Artech House, Norwood, MA, USA, 1992. ISBN 9780-89006-517-4. Cited on page 89. 
[10] Y. Bar-Shalom. Update with out-of-sequence measurements in tracking: exact solution. IEEE Transactions on Aerospace and Electronic Systems, 38(3):769-777, July 2002. Cited on page 15.

[11] Y. Bar-Shalom and T. Fortmann. Tracking and Data Association. Academic Press, San Diego, CA, USA, 1988. ISBN 978-0-12-079760-8. Cited on pages $9,34,89$, and 98 .

[12] Y. Bar-Shalom and X. R. Li. Multitarget-multisensor Tracking: Principles and Techniques. YBS Publishing, Storrs, CT, USA, 1995. ISBN 978-09648312-0-9. Cited on pages 9 and 89.

[13] Y. Bar-Shalom and E. Tse. Tracking in a cluttered environment with probabilistic data association. Automatica, 11(5):451-460, Sept. 1975. ISSN 0005-1098. doi:10.1016/0005-1098(75)90021-7. Cited on page 98.

[14] Y. Bar-Shalom, X. Li, and T. Kirubarajan. Estimation with Applications to Tracking and Navigation: Theory Algorithms and Software. Wiley, New York, NY, USA, 2004. ISBN 978-0-471-46521-8. Cited on pages 9 and 89.

[15] Y. Bar-Shalom, S. S. Blackman, and R. J. Fitzgerald. Dimensionless score function for multiple hypothesis tracking. IEEE Transactions on Aerospace and Electronic Systems, 43(1):392-400, Jan. 2007. ISSN 00189251. doi:10.1109/TAES.2007.357141. Cited on pages 91 and 92.

[16] L. E. Baum, T. Petrie, G. Soules, and N. Weiss. A maximization technique occurring in the statistical analysis of probabilistic functions of Markov chains. The Annals of Mathematical Statistics, 41(1):164-171, Feb. 1970. doi:10.1214/aoms/1177697196. Cited on page 65.

[17] M. Baum and U. D. Hanebeck. Random hypersurface models for extended object tracking. In Proceedings of the IEEE International Symposium on Signal Processing and Information Technology, pages 178-183, Ajman, UAE, Dec. 2009. doi:10.1109/ISSPIT.2009.5407526. Cited on page 35.

[18] B. M. Bell and F. W. Cathey. The iterated Kalman filter update as a GaussNewton method. IEEE Transactions on Automatic Control, 38(2):294-297, Feb. 1993. ISSN 0018-9286. doi:10.1109/9.250476. Cited on pages 58 and 60 .

[19] N. Bergman and F. Gustafsson. Three statistical batch algorithms for tracking manoeuvring targets. In Proceedings of the 1999 European Control Conference, pages 3082-3087, Karlsruhe, Germany, Aug. 1999. Cited on page 65.

[20] D. Bertsekas. Linear Network Optimization: Algorithms and Codes. MIT Press, Cambridge, MA, USA, 1991. ISBN 978-0-262-02334-4. Cited on page 95.

[21] D. P. Bertsekas. The auction algorithm for assignment and other network 
flow problems: A tutorial. Interfaces, 20(4):133-149, July 1990. ISSN 00922102. Cited on page 95.

[22] A. Bhattacharyya. On a measure of divergence between two statistical populations defined by their probability distributions. Bulletin of Calcutta Mathematical Society, 35(1):99-109, 1943. Cited on page 99.

[23] G. Bianco, M. Ilieva, C. Veibäck, K. Öfjäll, A. Gadomska, G. Hendeby, M. Felsberg, F. Gustafsson, and S. Åkesson. Emlen-funnel experiments revisited: methods update for studying compass orientation in songbirds. Ecology and Evolution, 6(19):6930-6942, 2016. ISSN 2045-7758. doi:10.1002/ece3.2383. Cited on pages $3,12,19,32,48,50,55,65,67$, and 68 .

[24] S. S. Blackman. Multiple-target Tracking with Radar Applications. Artech House, Norwood, MA, USA, 1986. ISBN 978-0-89006-179-4. Cited on pages 9,92 , and 95 .

[25] S. S. Blackman and R. Popoli. Design and Analysis of Modern Tracking Systems. Artech House, Norwood, MA, USA, 1999. ISBN 978-1-58053006-4. Cited on pages 9, 10, 89, 90, 91, 92, 93, 95, 98, and 107.

[26] H. A. P. Blom. An efficient filter for abruptly changing systems. In Proceedings of the 23rd IEEE Conference on Decision and Control, pages 656-658, Las Vegas, NV, USA, Dec. 1984. doi:10.1109/CDC.1984.272089. Cited on pages 10 and 65 .

[27] B. J. Boom, P. X. Huang, C. Spampinato, S. Palazzo, J. He, C. Beyan, E. Beauxis-Aussalet, J. van Ossenbruggen, G. Nadarajan, J. Y. Chen-Burger, D. Giordano, L. Hardman, F.-P. Lin, and R. B. Fisher. Long-term underwater camera surveillance for monitoring and analysis of fish populations. In Proceedings of the Workshop on Visual Observation and Analysis of Animal and Insect Behavior, pages 1-4, Tsukuba, Japan, Nov. 2012. Cited on page 12 .

[28] J. Y. Bouguet. Camera calibration toolbox for Matlab. http:// www.vision.caltech.edu/bouguetj/calib_doc/, 2010. Accessed: 2016-10-17. Cited on page 41.

[29] M. D. Breitenstein, F. Reichlin, B. Leibe, E. Koller-Meier, and L. Van Gool. Online multiperson tracking-by-detection from a single, uncalibrated camera. IEEE Transactions on Pattern Analysis and Machine Intelligence, 33 (9):1820-1833, Sept. 2011. ISSN 0162-8828. doi:10.1109/TPAMI.2010.232. Cited on page 47.

[30] M. Briers, A. Doucet, and S. Maskell. Smoothing algorithms for state-space models. Annals of the Institute of Statistical Mathematics, 62(1):61-89, 2009. ISSN 1572-9052. doi:10.1007/s10463-009-0236-2. Cited on page 59.

[31] C. Buragohain and S. Suri. Quantiles on streams. In Encyclopedia of 
Database Systems, pages 2235-2240. Springer US, 2009. ISBN 978-0-38739940-9. Cited on pages 45 and 48.

[32] H. R. Byrd, C. J. Gilbert, and J. Nocedal. A trust region method based on interior point techniques for nonlinear programming. Mathematical Programming, 89(1):149-185, $2000 . \quad$ ISSN 1436-4646. doi:10.1007/PL00011391. Cited on pages 42, 43, and 44.

[33] E. J. Candès, X. Li, Y. Ma, and J. Wright. Robust principal component analysis? J. ACM, 58(3):11:1-11:37, May 2011. ISSN 0004-5411. doi:10.1145/1970392.1970395. Cited on page 12 .

[34] D. A. Castanón. New assignment algorithms for data association. In Proceedings of SPIE, volume 1698, pages 313-323, Aug. 1992. doi:10.1117/12.139398. Cited on page 95.

[35] F. Ceragioli, G. Lindmark, C. Veibäck, N. Wahlström, M. Lindfors, and C. Altafini. A bounded confidence model that preserves the signs of the opinions. In Proceedings of the 2016 European Control Conference, Aalborg, Denmark, June 2016. Not cited.

[36] C. B. Chang and M. Athans. State estimation for discrete systems with switching parameters. IEEE Transactions on Aerospace and Electronic Systems, 14(3):418-425, May 1978. ISSN 0018-9251. doi:10.1109/TAES.1978.308603. Cited on page 32.

[37] I. J. Cox and S. L. Hingorani. An efficient implementation of Reid's multiple hypothesis tracking algorithm and its evaluation for the purpose of visual tracking. IEEE Transactions on Pattern Analysis and Machine Intelligence, 18(2):138-150, Feb. 1996. ISSN 0162-8828. doi:10.1109/34.481539. Cited on page 95.

[38] I. J. Cox and M. L. Miller. On finding ranked assignments with application to multitarget tracking and motion correspondence. IEEE Transactions on Aerospace and Electronic Systems, 31(1):486-489, Jan. 1995. ISSN 00189251. doi:10.1109/7.366332. Cited on page 95.

[39] W. C. Davidon. Variable metric method for minimization. Technical Report ANL-5990, Argonne National Laboratory, Argonne, IL, USA, 1959. Cited on page 83 .

[40] A. P. Dempster, N. M. Laird, and D. B. Rubin. Maximum likelihood from incomplete data via the EM algorithm. Journal of the Royal Statistical Society, Series B, 39(1):1-38, 1977. Cited on pages 15, 45, and 65.

[41] F. Devernay and O. Faugeras. Straight lines have to be straight: Automatic calibration and removal of distortion from scenes of structured enviroments. Machine Vision and Applications, 13(1):14-24, Aug. 2001. ISSN 0932-8092. doi:10.1007/PL00013269. Cited on page 44. 
[42] A. Doucet, A. Logothetis, and V. Krishnamurthy. Stochastic sampling algorithms for state estimation of jump Markov linear systems. IEEE Transactions on Automatic Control, 45(1):188-202, Jan. 2000. ISSN 0018-9286. Cited on page 85 .

[43] O. E. Drummond, D. A. Castanón, and M. S. Bellovin. Comparison of 2-D assignment algorithms for sparse, rectangular, floating point, cost matrices. Journal of the SDI Panels on Tracking, 4:81-97, Dec. 1990. Cited on page 95.

[44] S. T. Emlen. Migration: orientation and navigation. In D. S. Farner and J. R. King, editors, Avian Biology, pages 129-219. Academic Press, Amsterdam, Netherlands, 1975. ISBN 978-0-12-249405-5. Cited on page 13.

[45] S. T. Emlen and J. T. Emlen. A technique for recording migratory orientation of captive birds. Auk, 83(3):361-367, July 1966. doi:10.2307/4083048. Cited on pages 2, 3, and 13 .

[46] F. Eng and F. Gustafsson. Identification with stochastic sampling time jitter. Automatica, 44:637-646, Mar. 2008. doi:10.1016/j.automatica.2007.06.018. Cited on page 14.

[47] A. Flodell and C. Christensson. Wildlife surveillance using a UAV and thermal imagery. Master's thesis, Linköping University, Linköping, Sweden, June 2016. Cited on page 14.

[48] P.-E. Forssén. Low and Medium Level Vision using Channel Representations. Dissertation no. 858, Linköping University, Linköping, Sweden, Mar. 2004. Cited on page 48 .

[49] T. Fortmann, Y. Bar-Shalom, and M. Scheffe. Sonar tracking of multiple targets using joint probabilistic data association. IEEE Journal of Oceanic Engineering, 8(3):173-184, July 1983. ISSN 0364-9059. doi:10.1109/JOE.1983.1145560. Cited on page 98.

[50] D. Fraser and J. Potter. The optimum linear smoother as a combination of two optimum linear filters. IEEE Transactions on Automatic Control, 14 (4):387-390, Aug. 1969. ISSN 0018-9286. Cited on page 76.

[51] N. Friedman and S. Russell. Image segmentation in video sequences: A probabilistic approach. In Proceedings of the Thirteenth Conference on Uncertainty in Artificial Intelligence, pages 175-181, Providence, Rhode Island, 1997. Cited on page 12.

[52] W. Friedman, S. Potter, E. Hutchins, C. Johnson, M. Krtzen, and R. Connor. Three aerial technologies for recording subsurface behaviors among wild bottlenose dolphins (Tursiops sp.). In Proceedings of the 20th Biennial Conference on Marine Mammals, Dunedin, New Zealand, 2014. Cited on page 12 .

[53] A. Gelman, J. Carlin, H. Stern, D. Dunson, A. Vehtari, and D. Rubin. 
Bayesian Data Analysis. CRC Press, Boca Raton, FL, USA, third edition, 2014. ISBN 978-1-4398-4095-5. Cited on pages 35 and 72.

[54] C. N. Georghiades and D. L. Snyder. The expectation-maximization algorithm for symbol unsynchronized sequence detection. IEEE Transactions on Communications, 39(1):54-61, Jan. 1991. Cited on page 15.

[55] K. Gilholm and D. Salmond. Spatial distribution model for tracking extended objects. IEE Proceedings - Radar, Sonar and Navigation, 152(5): 364-371, Oct. 2005. ISSN 1350-2395. doi:10.1049/ip-rsn:20045114. Cited on page 35 .

[56] L. F. Gonzalez, G. A. Montes, E. Puig, S. Johnson, K. Mengersen, and K. J. Gaston. Unmanned aerial vehicles (UAVs) and artificial intelligence revolutionizing wildlife monitoring and conservation. Sensors, 16(1):97-114, Jan. 2016. ISSN 1424-8220. doi:10.3390/s16010097. Cited on page 14.

[57] R. C. Gonzalez and R. E. Woods. Digital Image Processing. Pearson Prentice Hall, Upper Saddle River, NJ, USA, third edition, 2008. ISBN 978-013-168728-8. Cited on pages $45,47,49$, and 51.

[58] N. J. Gordon, D. J. Salmond, and A. F. M. Smith. Novel approach to nonlinear/non-Gaussian Bayesian state estimation. IEE Proceedings on Radar and Signal Processing,, 140(2):107-113, Apr. 1993. ISSN 0956-375X. Cited on page 10 .

[59] K. Granström and U. Orguner. A PHD filter for tracking multiple extended targets using random matrices. IEEE Transactions on Signal Processing, 60(11):5657-5671, Nov. 2012. ISSN 1053-587X. doi:10.1109/TSP.2012.2212888. Cited on pages 34, 35, and 73.

[60] K. Granström, C. Lundquist, and U. Orguner. Tracking rectangular and elliptical extended targets using laser measurements. In Proceedings of the 14th International Conference on Information Fusion, Chicago, IL, USA, July 2011. Cited on page 35.

[61] M. S. Grewal and A. P. Andrew. Kalman Filtering: Theory and Practice. Prentice Hall, Englewood Cliffs, New Jersey, USA, 1993. ISBN 978-0-13211335-9. Cited on pages 10 and 55.

[62] S. Gunnarsson, Y. Jung, C. Veibäck, and T. Glad. IO (implement and operate) first - an alternative way to approach the automatic control subject. In Proceedings of 5:e Utvecklingskonferensen för Sveriges ingenjörsutbildningar, Uppsala, Sweden, Feb. 2016. Not cited.

[63] F. Gustafsson. Statistical Sensor Fusion. Studentlitteratur, Lund, Sweden, second edition, 2012. ISBN 978-91-44-07732-1. Cited on pages 10, 59, 61, and 64 .

[64] J. Hannah. A geometric approach to determinants. The American 
Mathematical Monthly, 103(5):401-409, May 1996. ISSN 0002-9890. doi:10.2307/2974931. Cited on page 74 .

[65] L.-A. Hansson and S. Åkesson, editors. Animal Movement Across Scales. Oxford University Press, Oxford, UK, 2014. ISBN 978-0-19-967718-4. Cited on page 13.

[66] R. Hartley and A. Zisserman. Multiple View Geometry in Computer Vision. Cambridge University Press, Cambridge, UK, second edition, 2003. ISBN 978-0-521-54051-3. Cited on page 39.

[67] D. L. Herzing. Vocalizations and associated underwater behavior of freeranging Atlantic spotted dolphins, Stenella frontalis and bottlenose dolphins, Tursiops truncatus. Aquatic Mammals, 22(2):61-79, 1996. ISSN 0167-5427. Cited on page 12.

[68] G. Icaza and R. Jones. A state-space EM algorithm for longitudinal data. Journal of Time Series Analysis, 20(5):537-550, 1999. ISSN 1467-9892. Cited on page 15.

[69] M. Israel. A UAV-based roe deer fawn detection system. In The International Archives of the Photogrammetry, Remote Sensing and Spatial Information Sciences, volume 3822, pages 51-55, Zurich, Switzerland, Sept. 2011. doi:10.5194/isprsarchives-XXXVIII-1-C22-51-2011. Cited on page 14.

[70] G. P. Jones IV, L. G. Pearlstine, and H. F. Percival. An assessment of small unmanned aerial vehicles for wildlife research. Wildlife Society Bulletin, 34(3):750-758, 2006. ISSN 1938-5463. doi:10.2193/00917648(2006)34[750:AAOSUA]2.0.CO;2. Cited on page 14.

[71] R. Jonker and A. Volgenant. A shortest augmenting path algorithm for dense and sparse linear assignment problems. Computing, 38(4):325-340, Dec. 1987. ISSN 1436-5057. doi:10.1007/BF02278710. Cited on page 95.

[72] S. J. Julier and J. K. Uhlmann. Unscented filtering and nonlinear estimation. Proceedings of the IEEE, 92(3):401-422, Mar. 2004. ISSN 0018-9219. doi:10.1109/JPROC.2003.823141. Cited on pages 10 and 58.

[73] S. J. Julier, J. K. Uhlmann, and H. F. Durrant-Whyte. A new method for the nonlinear transformation of means and covariances in filters and estimators. IEEE Transactions on Automatic Control, 45(3):477-482, Mar. 2000. ISSN 0018-9286. Cited on pages 10 and 52.

[74] R. E. Kalman. A new approach to linear filtering and prediction problems. Transactions of the ASME-Journal of Basic Engineering, 82(Series D):3545,1960 . Cited on pages 10,55 , and 56.

[75] J. Karnowski, E. Hutchins, and C. Johnson. Dolphin detection and tracking. In Proceedings of the IEEE Winter Applications and Computer 
Vision Workshops, pages 51-56, Waikoloa Beach, HI, USA, Jan. 2015. doi:10.1109/WACVW.2015.10. Cited on page 12.

[76] S. M. Kay. Fundamentals of Statistical Signal Processing: Estimation Theory. Prentice Hall, Englewood Cliffs, NJ, USA, 1993. ISBN 978-0-13345711-7. Cited on pages 10, 55, 77, 80, 82, and 87.

[77] O. Khatib. Real-time obstacle avoidance for manipulators and mobile robots. The International Journal of Robotics Research, 5(1):90-98, Spring 1986. Cited on page 24.

[78] G. Kitagawa. Non-Gaussian state-space modeling of nonstationary time series. Journal of the American Statistical Association, 82(400):1032-1041, Dec. 1987. ISSN 0162-1459. doi:10.2307/2289375. Cited on page 56 .

[79] J. W. Koch. Bayesian approach to extended object and cluster tracking using random matrices. IEEE Transactions on Aerospace and Electronic Systems, 44(3):1042-1059, July 2008. ISSN 0018-9251. Cited on pages 35, 71,72 , and 73 .

[80] H. W. Kuhn. The Hungarian method for the assignment problem. Naval Research Logistics Quarterly, 2(1-2):83-97, Mar. 1955. doi:10.1002/nav.3800020109. Cited on page 95.

[81] R. X. Li and V. P. Jilkov. Survey of maneuvering target tracking. Part I: Dynamic models. IEEE Transactions on Aerospace and Electronic Systems, 39(4):1333-1364, Oct. 2003. Cited on pages 21, 22, and 27.

[82] L. Ljung. System Identification: Theory for the User. Prentice Hall, Upper Saddle River, NJ, USA, 1999. ISBN 978-0-13-656695-3. Cited on page 20.

[83] A. Logothetis and V. Krishnamurthy. Expectation maximization algorithms for MAP estimation of jump Markov linear systems. IEEE Transactions on Signal Processing, 47(8):2139-2156, Aug. 1999. ISSN 1053-587X. doi:10.1109/78.774753. Cited on pages 65 and 85 .

[84] Y. Ma, S. Soatto, J. Kosecka, and S. Sastry. An Invitation to 3-D Vision. Springer, New York, NY, USA, 2004. ISBN 978-0-387-00893-6. Cited on pages 39,40 , and 41 .

[85] J. B. MacQueen. Some methods for classification and analysis of multivariate observations. In Proceedings of the Fifth Berkeley Symposium on Mathematical Statistics and Probability, volume 1, pages 281-297, Berkeley, CA, USA, 1967. Cited on page 51.

[86] R. P. S. Mahler. Statistical Multisource-Multitarget Information Fusion. Artech House, Norwood, MA, USA, 2007. ISBN 978-1-59693-092-6. Cited on pages 9 and 89 .

[87] J. Martin, H. H. Edwards, M. A. Burgess, H. F. Percival, D. E. Fagan, B. E. Gardner, J. G. Ortega-Ortiz, P. G. Ifju, B. S. Evers, and T. J. Rambo. Estimating distribution of hidden objects with drones: From tennis balls to 
manatees. PLoS ONE, 7(6), June 2012. doi:10.1371/journal.pone.0038882. Cited on page 14.

[88] H. Mouritsen, G. Feenders, M. Liedvogel, and W. Kropp. Migratory birds use head scans to detect the direction of the earth's magnetic field. Current Biology, 14(21):1946-1949, 2004. ISSN 0960-9822. doi:10.1016/j.cub.2004.10.025. Cited on page 13 .

[89] R. Muheim, I. Henshaw, S. Sjöberg, and M. E. Deutschlander. Birdoritrack: a new video-tracking program for orientation research with migratory birds. Journal of Field Ornithology, 85(1):91-105, 2014. ISSN 15579263. doi:10.1111/jofo.12053. Cited on page 13.

[90] M. Mulero-Pázmány, R. Stolper, L. D. van Essen, J. J. Negro, and T. Sassen. Remotely piloted aircraft systems as a rhinoceros anti-poaching tool in africa. PLoS ONE, 9(1), Jan. 2014. doi:10.1371/journal.pone.0083873. Cited on page 14.

[91] K. G. Murty. An algorithm for ranking all the assignments in order of increasing cost. Operations Research, 16(3):682-687, May 1968. doi:10.1287/opre.16.3.682. Cited on page 95.

[92] A. Naranjo, A. A. Trindade, and G. Casella. Extending the state-space model to accommodate missing values in responses and covariates. Journal of the American Statistical Association, 108(501):202-216, 2013. Cited on page 15.

[93] J. Nocedal and S. Wright. Numerical Optimization. Springer, New York, NY, USA, second edition, 2006. ISBN 978-0-387-30303-1. Cited on pages 42 and 58.

[94] U. Orguner and F. Gustafsson. Particle filtering with propagation delayed measurements. In Proceedings of the 2010 IEEE Aerospace Conference, Big Sky, MT, USA, Mar. 2010. doi:10.1109/AERO.2010.5446679. Cited on page 15 .

[95] C. C. Paige and M. A. Saunders. Least squares estimation of discrete linear dynamic systems using orthogonal transformations. SIAM Journal on Numerical Analysis, 14(2):180-193, Apr. 1977. Cited on pages 60 and 76.

[96] P. W. Power and J. A. Schoonees. Understanding background mixture models for foreground segmentation. In Proceedings of Image and Vision Computing New Zealand, pages 267-271, Auckland, New Zealand, Nov. 2002. Cited on page 45 .

[97] H. E. Rauch, F. Tung, and C. T. Striebel. Maximum likelihood estimates of linear dynamic systems. AIAA Journal, 3(8):1445-1450, Aug. 1965. Cited on pages $10,60,75$, and 76 .

[98] D. B. Reid. An algorithm for tracking multiple targets. IEEE Transac- 
tions on Automatic Control, 24(6):843-854, Dec. 1979. ISSN 0018-9286. doi:10.1109/TAC.1979.1102177. Cited on page 95.

[99] W. J. Rugh. Linear System Theory. Prentice Hall, Upper Saddle River, NJ, USA, 1996. ISBN 978-0-13-441205-4. Cited on page 27.

[100] A. R. Runnalls. A Kullback-Leibler approach to Gaussian mixture reduction. IEEE Transactions on Aerospace and Electronic Systems, 43(3):989999, July 2007. Cited on page 99.

[101] S. Särkkä. Bayesian Filtering and Smoothing. Cambridge University Press, Cambridge, United Kingdom, 2013. ISBN 978-1-107-03065-7. Cited on pages $10,20,55,56,57$, and 59 .

[102] R. H. Shumway and D. S. Stoffer. An approach to time series smoothing and forecasting using the EM algorithm. Journal of Time Series Analysis, 3(4):253-264, 1982. ISSN 1467-9892. Cited on page 15.

[103] I. Skog and P. Handel. Effects of time synchronization errors in GNSSaided INS. In IEEE/ION Position, Location and Navigation Symposium, pages 82-88, May 2008. Cited on page 15.

[104] Smart Savannahs. http://wildlifesecurity.se/ smart-savannahs/. Accessed: 2016-10-17. Cited on page 11.

[105] G. L. Smith, S. F. Schmidt, and L. A. McGee. Application of Statistical Filter Theory to the Optimal Estimation of Position and Velocity on Board a Circumlunar Vehicle. National Aeronautics and Space Administration, Washington D. C., USA, 1962. Cited on pages 10, 55, and 57.

[106] C. Stauffer and W. E. L. Grimson. Adaptive background mixture models for real-time tracking. In Proceedings of the IEEE Computer Society Conference on Computer Vision and Pattern Recognition, volume 2, pages 22462252, Ft. Collins, CO, USA, June 1999. doi:10.1109/CVPR.1999.784637. Cited on pages 45 and 46 .

[107] L. D. Stone, R. L. Streit, T. L. Corwin, and K. L. Bell. Bayesian Multiple Target Tracking. Artech House, Norwood, MA, USA, second edition, 2014. ISBN 978-1-60807-553-9. Cited on page 89.

[108] L. Taycher, J. W. Fischer III, and T. Darrel. Incorporating object tracking feedback into background maintenance framework. In Proceedings of the Seventh IEEE Workshops on Application of Computer Vision, volume 2, pages 120-125, Breckenridge, CO, USA, Jan. 2005. doi:10.1109/ACVMOT.2005.63. Cited on page 47.

[109] J. Tugnait. Adaptive estimation and identification for discrete systems with markov jump parameters. IEEE Transactions on Automatic Control, 27(5): 1054-1065, Oct. 1982. ISSN 0018-9286. doi:10.1109/TAC.1982.1103061. Cited on page 10 . 
[110] J. C. van Gemert, C. R. Verschoor, P. Mettes, K. Epema, L. P. Koh, and S. Wich. Nature Conservation Drones for Automatic Localization and Counting of Animals, pages 255-270. Springer International Publishing, Cham, Switzerland, 2015. ISBN 978-3-319-16178-5. doi:10.1007/978-3319-16178-5_17. Cited on page 14.

[111] L. van Zonneveld. The importance of acoustic enrichment for bottlenose dolphins (Tursiops truncatus) in human care. Master's thesis, Linköping University, Linköping, Sweden, Mar. 2015. Cited on page 2.

[112] C. Veibäck, G. Hendeby, and F. Gustafsson. Tracking of dolphins in a basin using a constrained motion model. In Proceedings of the 18th International Conference on Information Fusion, pages 1330-1337, Washington D. C., USA, July 2015. Cited on pages 2, 12, 19, 24, 26, 30, 39, 48, 49, 89, $98,100,101,102,103$, and 105.

[113] C. Veibäck, G. Hendeby, and F. Gustafsson. On fusion of sensor measurements and observation with uncertain timestamp for target tracking. In Proceedings of the 19th International Conference on Information Fusion, pages 1268-1275, Heidelberg, Germany, July 2016. Cited on pages 6, 12, $19,36,55,75,78,79,81,84$, and 86 .

[114] C. Vermeulen, P. Lejeune, J. Lisein, P. Sawadogo, and P. Bouché. Unmanned aerial survey of elephants. PLoS ONE, 8(2), Feb. 2013. doi:10.1371/journal.pone.0054700. Cited on page 14 .

[115] B. N. Vo and W. K. Ma. The Gaussian mixture probability hypothesis density filter. IEEE Transactions on Signal Processing, 54(11):4091-4104, Nov. 2006. ISSN 1053-587X. Cited on page 9.

[116] N. Wahlström and E. Özkan. Extended target tracking using Gaussian processes. IEEE Transactions on Signal Processing, 63(16):4165-4178, Aug. 2015. ISSN 1053-587X. doi:10.1109/TSP.2015.2424194. Cited on page 35.

[117] A. Wald. Sequential tests of statistical hypotheses. The Annals of Mathematical Statistics, 16(2):117-186, June 1945. ISSN 0003-4851. Cited on page 92.

[118] H. G. Wallraff and O. G. Gelderloos. Experiments on migratory orientation of birds with simulated stellar sky and geomagnetic field: Method and preliminary results. Oikos, 30(2):207-215, 1978. doi:10.2307/3543480. Cited on page 13.

[119] A. C. Watts, J. H. Perry, S. E. Smith, M. A. Burgess, B. E. Wilkinson, Z. Szantoi, P. G. Ifju, and H. F. Percival. Small unmanned aircraft systems for lowaltitude aerial surveys. Journal of Wildlife Management, 74(7):1614-1619, 2010. ISSN 0022-541X. doi:10.2193/2009-425. Cited on page 14.

[120] W. Wiltschko and R. Wiltschko. Magnetic Orientation and Celestial Cues in Migratory Orientation, pages 16-37. Birkhäuser Basel, Basel, Switzerland, 1991. ISBN 978-3-0348-7208-9. Cited on page 13. 
[121] K. Zhang, X. R. Li, and Y. Zhu. Optimal update with out-of-sequence measurements. IEEE Transactions on Signal Processing, 53(6):1992-2004, June 2005. Cited on page 15.

[122] K. Zhang, L. Zhang, and M.-H. Yang. Real-time compressive tracking. In Proceedings of the 12th European Conference on Computer Vision, pages 864-877, Florence, Italy, 2012. doi:10.1007/978-3-642-33712-3_62. Cited on page 12 .

[123] Z. Zhang. Flexible camera calibration by viewing a plane from unknown orientations. In Proceedings of the Seventh IEEE International Conference on Computer Vision, volume 1, pages 666-673, Kerkyra, Greece, 1999. doi:10.1109/ICCV.1999.791289. Cited on page 41.

[124] Z. Zhang. A flexible new technique for camera calibration. IEEE Transactions on Pattern Analysis and Machine Intelligence, 22(11):1330-1334, Nov. 2000. ISSN 0162-8828. doi:10.1109/34.888718. Cited on page 44. 


\section{Licentiate Theses \\ Division of Automatic Control \\ Linköping University}

P. Andersson: Adaptive Forgetting through Multiple Models and Adaptive Control of Car Dynamics. Thesis No. 15, 1983.

B. Wahlberg: On Model Simplification in System Identification. Thesis No. 47, 1985.

A. Isaksson: Identification of Time Varying Systems and Applications of System Identification to Signal Processing. Thesis No. 75, 1986.

G. Malmberg: A Study of Adaptive Control Missiles. Thesis No. 76, 1986.

S. Gunnarsson: On the Mean Square Error of Transfer Function Estimates with Applications to Control. Thesis No. 90, 1986.

M. Viberg: On the Adaptive Array Problem. Thesis No. 117, 1987.

K. Ståhl: On the Frequency Domain Analysis of Nonlinear Systems. Thesis No. 137, 1988.

A. Skeppstedt: Construction of Composite Models from Large Data-Sets. Thesis No. 149, 1988.

P. A. J. Nagy: MaMiS: A Programming Environment for Numeric/Symbolic Data Processing. Thesis No. 153, 1988.

K. Forsman: Applications of Constructive Algebra to Control Problems. Thesis No. 231, 1990.

I. Klein: Planning for a Class of Sequential Control Problems. Thesis No. 234, 1990.

F. Gustafsson: Optimal Segmentation of Linear Regression Parameters. Thesis No. 246, 1990.

H. Hjalmarsson: On Estimation of Model Quality in System Identification. Thesis No. 251, 1990.

S. Andersson: Sensor Array Processing; Application to Mobile Communication Systems and Dimension Reduction. Thesis No. 255, 1990.

K. Wang Chen: Observability and Invertibility of Nonlinear Systems: A Differential Algebraic Approach. Thesis No. 282, 1991.

J. Sjöberg: Regularization Issues in Neural Network Models of Dynamical Systems. Thesis No. 366, 1993.

P. Pucar: Segmentation of Laser Range Radar Images Using Hidden Markov Field Models. Thesis No. 403, 1993.

H. Fortell: Volterra and Algebraic Approaches to the Zero Dynamics. Thesis No. 438, 1994.

T. McKelvey: On State-Space Models in System Identification. Thesis No. 447, 1994.

T. Andersson: Concepts and Algorithms for Non-Linear System Identifiability. Thesis No. 448, 1994.

P. Lindskog: Algorithms and Tools for System Identification Using Prior Knowledge. Thesis No. 456, 1994.

J. Plantin: Algebraic Methods for Verification and Control of Discrete Event Dynamic Systems. Thesis No. 501, 1995.

J. Gunnarsson: On Modeling of Discrete Event Dynamic Systems, Using Symbolic Algebraic Methods. Thesis No. 502, 1995.

A. Ericsson: Fast Power Control to Counteract Rayleigh Fading in Cellular Radio Systems. Thesis No. 527, 1995.

M. Jirstrand: Algebraic Methods for Modeling and Design in Control. Thesis No. 540, 1996.

K. Edström: Simulation of Mode Switching Systems Using Switched Bond Graphs. Thesis No. 586, 1996. 
J. Palmqvist: On Integrity Monitoring of Integrated Navigation Systems. Thesis No. 600, 1997.

A. Stenman: Just-in-Time Models with Applications to Dynamical Systems. Thesis No. 601, 1997.

M. Andersson: Experimental Design and Updating of Finite Element Models. Thesis No. 611, 1997.

U. Forssell: Properties and Usage of Closed-Loop Identification Methods. Thesis No. 641, 1997.

M. Larsson: On Modeling and Diagnosis of Discrete Event Dynamic systems. Thesis No. 648, 1997.

N. Bergman: Bayesian Inference in Terrain Navigation. Thesis No. 649, 1997.

V. Einarsson: On Verification of Switched Systems Using Abstractions. Thesis No. 705, 1998.

J. Blom, F. Gunnarsson: Power Control in Cellular Radio Systems. Thesis No. 706, 1998.

P. Spångéus: Hybrid Control using LP and LMI methods - Some Applications. Thesis No. 724, 1998.

M. Norrlöf: On Analysis and Implementation of Iterative Learning Control. Thesis No. 727, 1998.

A. Hagenblad: Aspects of the Identification of Wiener Models. Thesis No. 793, 1999.

F. Tjärnström: Quality Estimation of Approximate Models. Thesis No. 810, 2000.

C. Carlsson: Vehicle Size and Orientation Estimation Using Geometric Fitting. Thesis No. 840, 2000.

J. Löfberg: Linear Model Predictive Control: Stability and Robustness. Thesis No. 866, 2001.

O. Härkegård: Flight Control Design Using Backstepping. Thesis No. 875, 2001.

J. Elbornsson: Equalization of Distortion in A/D Converters. Thesis No. 883, 2001.

J. Roll: Robust Verification and Identification of Piecewise Affine Systems. Thesis No. 899, 2001.

I. Lind: Regressor Selection in System Identification using ANOVA. Thesis No. 921, 2001.

R. Karlsson: Simulation Based Methods for Target Tracking. Thesis No. 930, 2002.

P.-J. Nordlund: Sequential Monte Carlo Filters and Integrated Navigation. Thesis No. 945, 2002.

M. Östring: Identification, Diagnosis, and Control of a Flexible Robot Arm. Thesis No. 948, 2002.

C. Olsson: Active Engine Vibration Isolation using Feedback Control. Thesis No. 968, 2002.

J. Jansson: Tracking and Decision Making for Automotive Collision Avoidance. Thesis No. 965, 2002.

N. Persson: Event Based Sampling with Application to Spectral Estimation. Thesis No. 981, 2002.

D. Lindgren: Subspace Selection Techniques for Classification Problems. Thesis No. 995, 2002.

E. Geijer Lundin: Uplink Load in CDMA Cellular Systems. Thesis No. 1045, 2003.

M. Enqvist: Some Results on Linear Models of Nonlinear Systems. Thesis No. 1046, 2003.

T. Schön: On Computational Methods for Nonlinear Estimation. Thesis No. 1047, 2003.

F. Gunnarsson: On Modeling and Control of Network Queue Dynamics. Thesis No. 1048, 2003.

S. Björklund: A Survey and Comparison of Time-Delay Estimation Methods in Linear Systems. Thesis No. 1061, 2003. 
M. Gerdin: Parameter Estimation in Linear Descriptor Systems. Thesis No. 1085, 2004.

A. Eidehall: An Automotive Lane Guidance System. Thesis No. 1122, 2004.

E. Wernholt: On Multivariable and Nonlinear Identification of Industrial Robots. Thesis No. 1131, 2004.

J. Gillberg: Methods for Frequency Domain Estimation of Continuous-Time Models. Thesis No. 1133, 2004.

G. Hendeby: Fundamental Estimation and Detection Limits in Linear Non-Gaussian Systems. Thesis No. 1199, 2005.

D. Axehill: Applications of Integer Quadratic Programming in Control and Communication. Thesis No. 1218, 2005.

J. Sjöberg: Some Results On Optimal Control for Nonlinear Descriptor Systems. Thesis No. 1227, 2006.

D. Törnqvist: Statistical Fault Detection with Applications to IMU Disturbances. Thesis No. 1258, 2006.

H. Tidefelt: Structural algorithms and perturbations in differential-algebraic equations. Thesis No. 1318, 2007.

S. Moberg: On Modeling and Control of Flexible Manipulators. Thesis No. 1336, 2007.

J. Wallén: On Kinematic Modelling and Iterative Learning Control of Industrial Robots. Thesis No. 1343, 2008.

J. Harju Johansson: A Structure Utilizing Inexact Primal-Dual Interior-Point Method for Analysis of Linear Differential Inclusions. Thesis No. 1367, 2008.

J. D. Hol: Pose Estimation and Calibration Algorithms for Vision and Inertial Sensors. Thesis No. 1370, 2008.

H. Ohlsson: Regression on Manifolds with Implications for System Identification. Thesis No. 1382, 2008.

D. Ankelhed: On low order controller synthesis using rational constraints. Thesis No. 1398, 2009.

P. Skoglar: Planning Methods for Aerial Exploration and Ground Target Tracking. Thesis No. 1420, 2009.

C. Lundquist: Automotive Sensor Fusion for Situation Awareness. Thesis No. 1422, 2009.

C. Lyzell: Initialization Methods for System Identification. Thesis No. 1426, 2009.

R. Falkeborn: Structure exploitation in semidefinite programming for control. Thesis No. 1430, 2010.

D. Petersson: Nonlinear Optimization Approaches to $\mathcal{H}_{2}$-Norm Based LPV Modelling and Control. Thesis No. 1453, 2010.

Z. Sjanic: Navigation and SAR Auto-focusing in a Sensor Fusion Framework. Thesis No. 1464, 2011.

K. Granström: Loop detection and extended target tracking using laser data. Thesis No. 1465, 2011.

J. Callmer: Topics in Localization and Mapping. Thesis No. 1489, 2011.

F. Lindsten: Rao-Blackwellised particle methods for inference and identification. Thesis No. 1480, 2011.

M. Skoglund: Visual Inertial Navigation and Calibration. Thesis No. 1500, 2011.

S. Khoshfetrat Pakazad: Topics in Robustness Analysis. Thesis No. 1512, 2011.

P. Axelsson: On Sensor Fusion Applied to Industrial Manipulators. Thesis No. 1511, 2011.

A. Carvalho Bittencourt: On Modeling and Diagnosis of Friction and Wear in Industrial Robots. Thesis No. 1516, 2012.

P. Rosander: Averaging level control in the presence of frequent inlet flow upsets. Thesis No. 1527, 2012. 
N. Wahlström: Localization using Magnetometers and Light Sensors. Thesis No. 1581, 2013.

R. Larsson: System Identification of Flight Mechanical Characteristics. Thesis No. 1599, 2013.

Y. Jung: Estimation of Inverse Models Applied to Power Amplifier Predistortion. Thesis No. 1605, 2013.

M. Syldatk: On Calibration of Ground Sensor Networks. Thesis No. 1611, 2013.

M. Roth: Kalman Filters for Nonlinear Systems and Heavy-Tailed Noise. Thesis No. 1613, 2013.

D. Simon: Model Predictive Control in Flight Control Design - Stability and Reference Tracking. Thesis No. 1642, 2014.

J. Dahlin: Sequential Monte Carlo for inference in nonlinear state space models. Thesis No. 1652, 2014.

M. Kok: Probabilistic modeling for positioning applications using inertial sensors. Thesis No. 1656, 2014.

J. Linder: Graybox Modelling of Ships Using Indirect Input Measurements. Thesis No. 1681, 2014.

G. Mathai: Direction of Arrival Estimation of Wideband Acoustic Wavefields in a Passive Sensing Environment. Thesis No. 1721, 2015.

I. Nielsen: On Structure Exploiting Numerical Algorithms for Model Predictive Control. Thesis No. 1727, 2015. 